\title{
Difusión caótica en sistemas Hamiltonianos casi-integrables
}

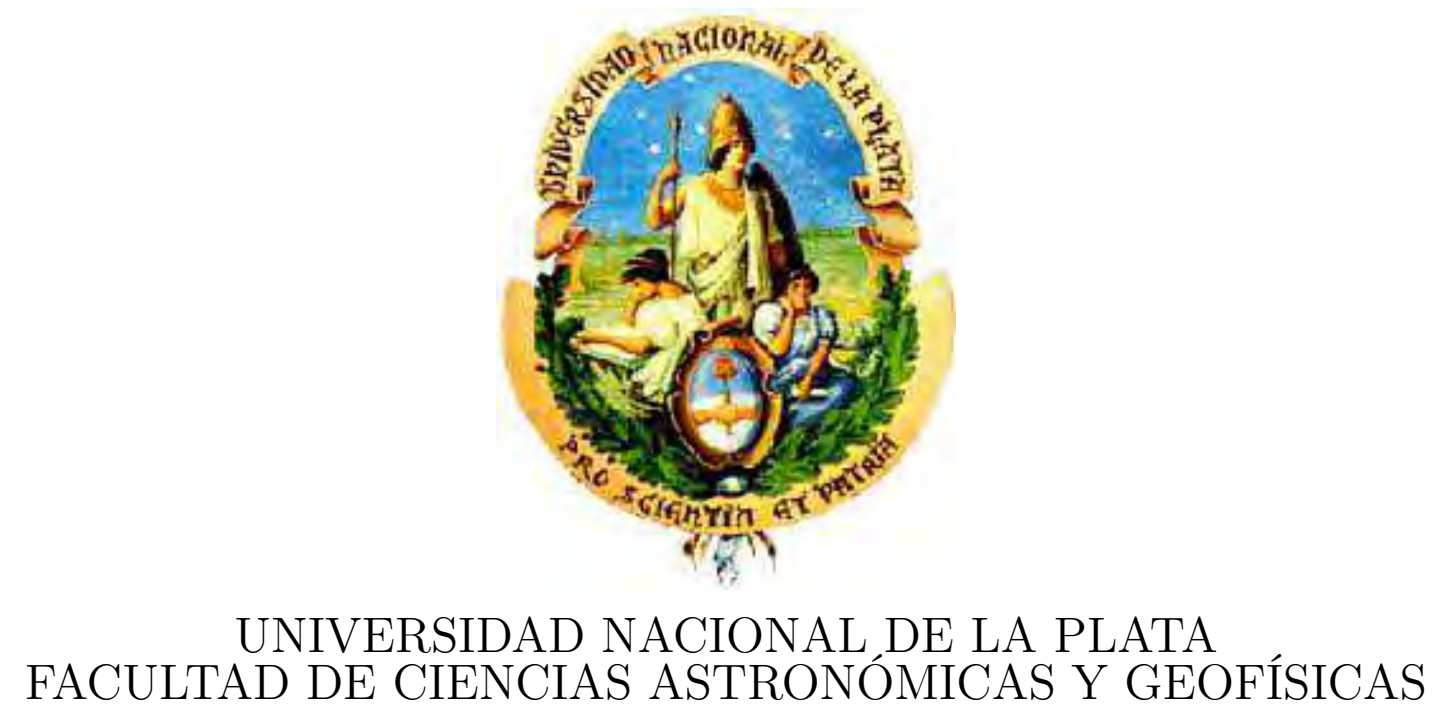

Tesis presentada por

Martín Federico Mestre

para obtener el grado de

DOCTOR EN ASTRONOMÍA

\author{
Director: Pablo M. Cincotta \\ Codirectora: Claudia M. Giordano
}

\author{
Integrantes del jurado: \\ Carlos Briozzo \\ Ricardo Cordeiro \\ Ana María Platzeck
}

Febrero, 2012 


\section{Resumen}

En esta tesis se avanza en el conocimiento de los procesos difusivos que tienen lugar en el espacio de acciones de sistemas Hamiltonianos casi-integrables. Fundamentalmente, se estudia la difusión de Arnold que tiene lugar a lo largo de una resonancia, considerando un flujo Hamiltoniano con tres grados de libertad cuya superficie de energía no perturbada es convexa en el espacio de acciones.

Además, se estudia la difusión en un mapa simpléctico 4D a priori inestable, sobre el cual se aplica un método semi-numérico para predecir aproximadamente el coeficiente de difusión asociado a una de las acciones, y se corrobora numéricamente que el proceso satisface una ecuación de Fokker-Planck. 


\section{Agradecimientos}

Durante el tiempo que llevó realizar este trabajo de tesis, he recibido ayuda y ánimo de mucha gente.

Primero, agradezco sinceramente a mis directores, Pablo Cincotta y Claudia Giordano, quienes me introdujeron en el tema de la difusión en sistemas Hamiltonianos. Ellos me han ayudado a utilizar la creatividad para lograr el objetivo de la tesis y a desarrollar mis propias herramientas. Siempre les voy a agradecer por haberme tenido paciencia en mi lento despertar hacia una actitud más pragmática hacia la ciencia y por su confianza en mí.

A lo largo de estos años de doctorado tuvimos muchas charlas, discusiones, acuerdos y desacuerdos, sobre dinámica, caos y difusión, que han resultado de una gran riqueza para mi formación. Durante toda la tesis recibí de ellos palabras de ánimo y valoración de mi trabajo y supieron señalarme mis puntos débiles.

Además, cuando el tiempo escaseaba en momentos próximos al cierre de la tesis, recibí una intensa ayuda de su parte para mejorar la cohesión y claridad de la tesis así como innumerables correcciones a la misma.

No sólo aprendí de ellos en el plano estrictamente científico, sinó que también en un plano en el que lo académico y lo social se mezclan: observándolos tuve la oportunidad de aprender la intensa tarea y dedicación que implica construir y llevar adelante un grupo de trabajo, determinando sus objetivos y prioridades, registrando sus necesidades y consiguiendo los recursos.

I deeply thank my Bologna advisor, Armando Bazzani, for introducing me in the theory of stochastic systems. I really appreciate that he searched for a system where I could study chaotic diffusion by applying some stochastic concepts. I also thank him for his patience at teaching me the concepts of event space and of noise realization which turned out to be really important in my research.

I also want to thank Giorgio Turchetti, for helping me in increasing my efficiency at work and for introducing me to many people with research interests similar to mine.

I am indebted to Davide Faranda for sharing his enthusiasm and pragmatism with me. The experience of working with him has been very fruitfull and helped me to develop scientific and team skills.

Agradezco a Christos Efthymiopoulos por explicarme sobre diversos aspectos matemáticos de la difusión y sobre aplicaciones astronómicas. Le agradezco también por su ayuda en uno de los experimentos de esta tesis y por su interés en que la termine a tiempo.

Agradezco al jurado, conformado por Ana María Platzeck, Carlos Briozzo y Ricardo Cordeiro, por las correcciones y sugerencias.

Esta tesis posee una gran componente numérica y, por lo tanto, las personas encargadas de mantener las herramientas computacionales con las que trabajé diariamente, han sido imprescindibles.

Le agradezco a Eduardo Suarez por mantener al cluster seminare. Le agradezco a Tito Viturro por mantener los clusters athena y mnemosyne, los cuales me acompañaron durante toda la tesis. Le agredezco a Pablo Santamaría por mantener mi cpu de escritorio. También le agradezco por haberme presentado al lenguage fortran-90, hecho que precipitó un cambio absolutamente beneficioso en mi estilo de programación. Les agradezco a los tres por mantener sólidos sistemas de backup, a los que tuve que recurrir en más de una oportunidad, y por darme 
soporte informático. También le agradezco a Federico Bareilles por su ayuda en cuestiones informáticas.

I thank Graziano Servizi and Andrea Sgattoni for all their valuable help in computational issues, specially regarding the two Bologna clusters: osmino and pedrillo.

Quiero agradecer a los otros dos becarios del grupo: Nicolás Maffione y Luciano Darriba, por las interesantes discusiones sobre dinámica, indicadores y difusión. En particular, le agradezco a Nico por pasarme referencias útiles sobre indicadores y le agradezco a Luciano por enseñarme a usar el integrador Taylor.

Quisiera poder agradecerle a todo profesor, doctor, doctorando y estudiante con el cual alguna vez charlé, intercambié alguna idea o le hice una pregunta, sea personalmente o vía email. Entre ellos, agradezco a Luis Benet, Carles Simó, Àngel Jorba, Giampaolo Cristadoro, Aleksei Chechkin, Tassos Bountis, Constantino Tsallis, Yannis Kominis, Pipi Vucetich, Tabaré Gallardo, Cristian Beaugé, Sylvio Ferraz-Mello, James Meiss, Romina Di Sisto, Gonzalo de Elía, Octavio Miloni, Juan Carlos Muzzio, Jorge Correa Otto, Martín Leiva, Hugo Folonier, Yamila Miguel, Octavio Guilera, Javier Martí, Mathew Katsanikas, Iván Shevchenko, Jorge Antezana, Eduardo Chiumiento, Jorge Solomin, Alberto Maltz y Gabriel Baglietto. Agradezco también a Mercedes Mosquera por su ayuda y a Sergio Cellone por el bibtex.

Gracias a todos los colegas del edificio de óptica; a quienes se ocuparon de equiparla con heladera, microondas y sillones, a quienes se encargaron del agua potable, a quienes gestionaron mejoras edilicias y a quienes rescataron alguna vez un gatito atrapado en las cañerías.

Agradezco a Alejandro Córsico por aclararme dudas sobre el reglamento de posgrado, a Chaves por su orientación sobre declaraciones juradas y al resto del personal de la Facultad.

Agradezco a Claudio Llinares por haberme ayudado, a lo largo de estos años, tanto en aspectos científicos como humanos. También le agradezco por compartir conmigo largas horas de estudio mientras estabamos en nuestra formación de grado.

Para enunciar todos los motivos por los cuales agradezco a mi amigo Carlos Argüelles debería agregar un capítulo más a esta tesis. Agradezco a Charly por todas las veces que estudiamos juntos, por todos esos momentos de concentración y por su terrible optimismo. Le agradezco también por aquellos años en que no estábamos graduados y quisimos iniciar un grupo de investigación casero llamado "A Orden Cero". Le agradezco por su frase: "supongamos que es fácil", que me ha sido de gran utilidad ante problemas que yo pensaba que no podía resolver. Y sobre todo, le agradezco por las experiencias humanas y sociales que compartimos juntos y que han contribuído en mi de la mejor manera.

Agradezco a Demian Slobinsky por su amistad y por compartir sus conocimientos de física. Agradezco a mi amigo Cristian Carrión por haberme ayudado a tomar valor para viajar a Italia. Agradezco a Javier Haboba, Victor Vera, Silvia Agogeri y José Bermudez por su amistad y por hacer que la estadía en Bolonia sea una alegre experiencia.

Agradezco a Felipe Wachlin, Alejandra Romero e Isabel Renedo que, junto con Nico y Luciano, me han alegrado cada día en la oficina, compartiendo almuerzos, mates y risas. Realmente han logrado que yo tuviera ganas de ir a trabajar todos los días.

Agradezco a todos mis amigos por los buenos momentos juntos. Gracias Vicky, Tomás, Yael, Fede, Juli, Yami, Juan, Fede Conte. Gracias Nico Salerno por regalarme una de las frases más sencillas y verdaderas que conocí: "Hay que poner a mover el carro, pués los melones se acomodan solos". Gracias Matus, Javi Benitez, Antonio, Darío y Vincent por las charlas 
profundas y las risas. Gracias Cristóbal, Polaco y Martín por esos viejos tiempos.

Gracias Nicolás Nessi por enseñarme zazen y por las charlas sobre física. Gracias al Dojo Zen de La Plata.

Agradezo a mis amigos de Neuquén: Gustavo, Diego, Roberto, Aníbal, Gonza y Javi, por acompañarme a la distancia. Muchas gracias Ricardo por estar siempre presente cada vez que vuelvo al Alto Valle, por las tardes en el Río Grande o en Las Bardas y por los viajes de mochileros.

Agradezco a Diana por ayudarme a ser feliz. Agradezco a toda mi familia por el amor, la fuerza y la confianza en mí. Gracias a mi madre por su frase: "la palabra construye realidad" y a mi padre por haberme hablado sobre el Universo cuando yo era pequeño.

Quiero agradecer con todo mi corazón a mi esposa Valeria Dascanio, por haberme acompañado con su amor durante toda esta etapa de mi vida, por entenderme, por ayudarme cuando yo no veía la salida y por festejar mis logros. Por esto y por muchas razones más, es que le dedico cada ecuación, letra y gráfico presente en esta tesis a mi Vale.

El trabajo desarrollado en esta tesis se ha financiado a través de una beca de CONICET. Agradezco a mis lugares de trabajo: el Instituto de Astrofísica de La Plata (CCT La Plata - CONICET, UNLP) y la Facultad de Ciencias Astronómicas y Geofísicas de la UNLP. The seven month stay at Bologna was economically supported with an Erasmus Mundus ECW Lot-16 (EADIC) grant. Finally, I thank the organizing committee of the Thessaloniki conference: "Nonlinear Dynamics and Complexity: Theory, Methods and Applications" (2010) for supplying me with free accomodation. 
VI

A Valeria 


\section{Índice general}

$\begin{array}{ll}\text { Prólogo } & 1\end{array}$

1. Introducción y marco teórico 3

1.1. Sistemas dinámicos . . . . . . . . . . . . . . . . 3

1.2. Sistemas dinámicos Hamiltonianos . . . . . . . . . . . . . . 4

1.2.1. Formalismo Hamiltoniano . . . . . . . . . . . . . . . . 4

1.2.2. Movimiento integrable y variables ángulo-acción . . . . . . . . . 4

1.2.3. Degeneración, resonancias y no-linealidad . . . . . . . . . . . 6

1.2.4. Sistemas Hamiltonianos casi-integrables y teoría perturbativa . . . . . 7

1.2.5. Formas normales . . . . . . . . . . . . . . . . 9

1.2.6. La teoría KAM . . . . . . . . . . . . . . . . . . . . 10

1.2.7. El péndulo simple . . . . . . . . . . . . . . . . . . . . 10

1.2.8. La capa estocástica . . . . . . . . . . . . . . . . . . . . 12

1.3. Difusión en el espacio de acciones . . . . . . . . . . . . . . . . . . . . 12

1.3.1. Coeficientes de difusión . . . . . . . . . . . . . . . . 12

1.3.2. Difusión y transporte . . . . . . . . . . . . . . . . 16

1.3.3. Permisos topológicos y dinámicos . . . . . . . . . . . . . . . 22

1.4. Teorías de la difusión . . . . . . . . . . . . . . . . . . . 25

1.4.1. Mecanismo de Arnold . . . . . . . . . . . . . . . . . 25

1.4.2. Difusión de Arnold . . . . . . . . . . . . . . . . . . . . 26

1.4.3. Teoría de Nekhoroshev . . . . . . . . . . . . . . . . . . . 26

1.4.4. Teoría de Chirikov para la difusión de Arnold . . . . . . . . . . . . . . . . . 27

1.4.5. Pumping estocástico . . . . . . . . . . . . . . 32

1.4.6. Teorías de origen estocástico . . . . . . . . . . . . . . 33

1.5. Modelos de difusión en astronomía . . . . . . . . . . . . . . . 34

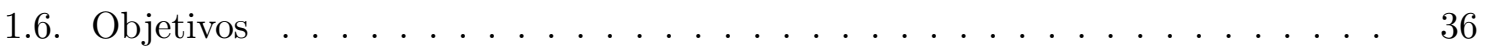

2. Indicadores de caos $\quad 39$

2.1. Exponentes Característicos de Lyapunov . . . . . . . . . . . . . . . . 39

2.2. Smaller Alignment Index . . . . . . . . . . . . . . . . . . . . . . . . . 40 
2.3. Fast Lyapunov Indicator . . . . . . . . . . . . . . . . . . . . . . . . . . . . . . 41

2.4. Mean Exponential Growth Factor of Nearby Orbits . . . . . . . . . . . . . . . 41

2.5. La relación entre FLI y MEGNO . . . . . . . . . . . . . . . . . . . . . . . . 42

2.5.1. Experimentos con el Mapa Standard. . . . . . . . . . . . . . . . . . 44

2.6. Visualización de estructura resonante . . . . . . . . . . . . . . . . . . . . . 46

2.7. Conclusión ......................... 46

3. La estructura resonante del flujo Hamiltoniano 49

3.1. El oscilador cuártico . . . . . . . . . . . . . . . . . . . . . 51

3.2. El modelo dinámico $2 \mathrm{DoF}$. . . . . . . . . . . . . . . . . . . . . 52

3.3. Resonancias a $\mathcal{O}(\epsilon)$. . . . . . . . . . . . . . . . . . . 53

3.4. Ancho de las resonancias a $\mathcal{O}(\epsilon)$. . . . . . . . . . . . . . . . . . . 54

3.5. Resonancias a $\mathcal{O}\left(\epsilon^{2}\right)$. . . . . . . . . . . . . . . . . . 56

3.6. Estimación numérica del valor crítico del parámetro perturbativo . . . . . . . 59

3.7. El modelo dinámico 3DoF . . . . . . . . . . . . . . . . . . . . . . . . . . . . 60

3.8. Resonancias a $\mathcal{O}(\epsilon)$. . . . . . . . . . . . . . . . . . . . . . . 62

3.9. Resonancias a $\mathcal{O}\left(\epsilon^{2}\right)$. . . . . . . . . . . . . . . . . . . 63

3.10. Estimación analítica del valor crítico del parámetro perturbativo para el siste-

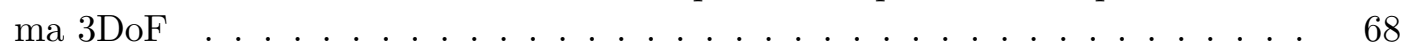

3.11. Conclusión ......................... . 69

4. Fenómeno difusivo en sistema Hamiltoniano 3DoF 71

4.1. El integrador simpléctico . . . . . . . . . . . . . . . . . . . . . . 71

4.2. La resonancia guía y su entorno . . . . . . . . . . . . . . . . . . . . . 73

4.3. Experimento $\mathscr{A}$. . . . . . . . . . . . . . . . . . . . 75

4.3.1. Los ensambles . . . . . . . . . . . . . . . . . . . . . 75

4.3.2. Las mediciones ....................... . . 78

4.4. Experimento $\mathscr{B}$. . . . . . . . . . . . . . . . . . . . 86

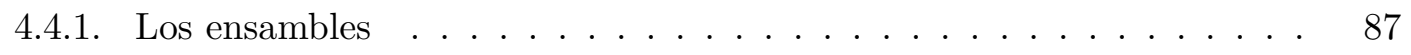

4.4.2. Las mediciones . . . . . . . . . . . . . . . . . 88

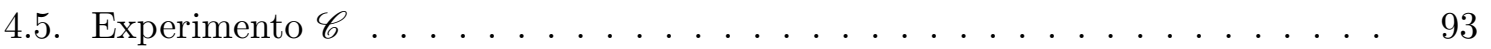

4.5.1. Los ensambles . . . . . . . . . . . . . . . . . 93

4.5.2. Las mediciones . . . . . . . . . . . . . . . . . . . . . 93

4.6. Conclusión ........................... . 96

5. El enfoque estocástico $\quad 99$

5.1. Un teorema de promediado . . . . . . . . . . . . . . . . . . . 100

5.2. Ejemplos estocásticos con ruidos Gaussianos . . . . . . . . . . . . . . . . . . 103

5.2.1. Ruido blanco . . . . . . . . . . . . . . . . . . . 105

5.2.2. Ruido generado mediante convolución de ruidos blancos. . . . . . . . . 105

5.2.3. Proceso Ornstein-Uhlenbeck . . . . . . . . . . . . . . . . 106

5.2.4. Ecuación de Langevin de un oscilador armónico amortiguado . . . . . 108

5.3. Perturbaciones deterministas . . . . . . . . . . . . . . . . . . . 111

5.3.1. Del formalismo estocástico a la simplecticidad . . . . . . . . . . . . . . 112

5.3.2. Generalización del método . . . . . . . . . . . . . . . . . . 114

5.4. Capa estocástica gruesa . . . . . . . . . . . . . . . . . . 116

5.4.1. Funciones de correlación . . . . . . . . . . . . . . . . 116 
5.4.2. Coeficientes de difusión . . . . . . . . . . . . . . . . . . . 118

5.4.3. La densidad de probabilidad . . . . . . . . . . . . . . . . 120

5.5. Capa estocástica delgada . . . . . . . . . . . . . . . . . . . . 120

5.5.1. Funciones de correlación . . . . . . . . . . . . . . . . . . . . . 121

5.5.2. Coeficientes de difusión . . . . . . . . . . . . . . . . . . . . . . . . . . 122

5.5.3. La densidad de probabilidad . . . . . . . . . . . . . . . 123

5.6. Conclusión . . . . . . . . . . . . . . . . . . 126

$\begin{array}{ll}\text { 6. Conclusiones y perspectivas } & 129\end{array}$

$\begin{array}{ll}\text { Bibliografía } & 131\end{array}$ 
Los sistemas Hamiltonianos son una herramienta de la Mecánica Clásica, que es uno de los paradigmas con el que se estudia la naturaleza. Muchos sistemas astrofísicos suelen ser modelados, total o parcialmente, por ecuaciones diferenciales Hamiltonianas. Entre los ejemplos se encuentran el Sistema Solar, sistemas planetarios en general, planetas con lunas, cúmulos estelares, galaxias, cúmulos de galaxias y todo otro objeto astronómico cuyas partes constitutivas estén relacionadas entre sí a través de la interacción gravitatoria.

La condición de no integrabilidad de los sistemas Hamiltonianos genéricos hace que los mismos sean una herramienta "incompleta". En las aplicaciones es generalmente necesario complementarlos con desarrollos perturbativos o simulaciones numéricas, dando ambos métodos información aproximada.

Los sistemas Hamiltonianos casi-integrables, han sido objeto de un creciente estudio desde los tiempos de Poincaré $(1854$ - 1912) con sus investigaciones del problema de tres cuerpos. Los mismos poseen un espacio de fases donde coexisten órbitas con diferentes niveles de caoticidad, con lo cual no es sencillo adoptar una descripción, o una metodología, adecuada para su estudio.

Aún persisten problemas abiertos acerca de la estabilidad, transporte y difusión en sistemas Hamiltonianos. A lo largo del tiempo, se han formulado muchas preguntas al respecto, la mayoría de las cuales no han sido respondidas.

Dada una condición inicial, caracterizada por los valores de las variables ángulo-acción: ¿cuál es la probabilidad de que después de un cierto intervalo de tiempo, la trayectoria haya alcanzado un dado entorno del espacio de acciones?, ¿cuál es el tiempo medio necesario para que la partícula de prueba se haya alejado, en una cierta cantidad, de su acción inicial?, ¿si se pudiese seguir la trayectoria durante un tiempo infinito, se llenaría densamente alguna porción del espacio de fases? Dado un ensamble de partículas con condiciones iniciales en un dado entorno del espacio de fases: ¿cuál será la dependencia temporal de su varianza o su desplazamiento cuadrático medio?, ¿dicha varianza crecerá más allá de cierta cota?, ¿es posible modelar macroscópicamente al ensamble mediante una función densidad de probabilidad?, ¿es posible escribir alguna ecuación cinética que gobierne la evolución de esta función?

Existen teorías de estos procesos de difusión caótica. Las mismas varían en las características de los sistemas sobre los cuales hacen predicciones y varían en el tipo de predicción que pueden hacer. La literatura al respecto es extensa y abarca trabajos con un amplio rango de 
enfoques. Existen trabajos netamente matemáticos, con formulación y demostración de teoremas, así como teorías semi-empíricas, basadas en experimentos numéricos o en herramientas matemáticas provenientes de áreas externas a la de sistemas dinámicos. A su vez, algunas teorías buscan mecanismos dinámicos capaces de producir el transporte, mientras que otras se ocupan de predecir cómo será la evolución de cantidades estadísticas, como son la varianza y las correlaciones entre las partículas de un ensamble. Dos de los conceptos dinámicos, cuyos efectos sobre la difusión (y el transporte) se buscan comprender con mayor profundidad en la actualidad son las interacciones entre resonancias que existen en los sistemas Hamiltonianos no lineales, y la geometría de las variedades hiperbólicas.

En esta tesis se avanza en el conocimiento de los procesos difusivos que tienen lugar en el espacio de acciones de sistemas Hamiltonianos casi-integrables. Fundamentalmente, se estudia la difusión de Arnold que tiene lugar a lo largo de una resonancia, considerando un flujo Hamiltoniano con tres grados de libertad cuya superficie de energía no perturbada es convexa en el espacio de acciones.

Además, se estudia la difusión en un mapa simpléctico 4D a priori inestable, sobre el cual se aplica un método semi-numérico para predecir aproximadamente el coeficiente de difusión asociado a una de las acciones, y se corrobora numéricamente que el proceso satisface una ecuación de Fokker-Planck.

Se aplicarán teorías analíticas, heurísticas, semi-numéricas y numéricas, algunas de ellas desarrolladas específicamente para estas investigaciones y otras desarrolladas con anterioridad.

Por todo lo expuesto, en la Introducción de este trabajo de tesis se brindará de manera extensa, pero a la vez lo más abreviada posible, todas las herramientas y el marco teórico preexistentes en el tema, al efecto de adentrarnos en el estudio de los procesos de difusión caótica en el espacio de fases de sistemas Hamiltonianos. 


\section{Introducción y marco teórico}

\subsection{Sistemas dinámicos}

Definimos a un sistema como una entidad abstracta matemática cuyo estado es capaz de ser descripto por un conjunto de variables que toman como valores números reales. El conjunto de estados accesibles al sistema será llamado espacio de fases. La dimensión de un sistema (o de su espacio de fases), notada $N_{d}$, es el mínimo número de variables necesarias para establecer unívocamente el estado del sistema.

Un sistema dinámico es un sistema cuyo estado puede cambiar en el tiempo dotado de una ley que determina unívocamente dicha evolución temporal. Se notará como $\mathbf{r}(t)$ o $\mathbf{r}_{t}$ al vector cuyas componentes $\left(r_{i}(t), i=1, \ldots, N_{d}\right)$ son las variables al tiempo $t$.

$\mathrm{El}$ espacio de fases puede ser discreto o continuo. En el primer caso, los estados que puede tener el sistema pertenecen a un conjunto finito o a un conjunto infinito numerable. En el segundo caso, los estados del sistema forman una variedad continua. En esta tesis sólo se trabajará con espacio de fases continuo.

El tiempo también puede tener carácter discreto o continuo. El primer tipo de sistema es llamado mapa o mapeo, mientras que el segundo es usualmente llamado flujo. Generalmente la ley arriba mencionada es representable por una ecuación que permite conocer el estado inmediatamente posterior, asumiendo conocido el actual. Esto significa que, en el caso de tiempo discreto, dada $\mathbf{r}_{t}$, habrá una fórmula determinista que nos dará $\mathbf{r}_{t+1}$, como:

$$
\mathbf{r}_{t+1}=\mathbf{f}\left(\mathbf{r}_{t}, t\right),
$$

donde $\mathbf{f}$ es una función cuyo codominio es $\mathbb{R}^{N_{d}}$ y puede o no depender explícitamente de $t$. En el caso de tiempo continuo, la mencionada ley es un sistema de $N_{d}$ ecuaciones diferenciales ordinarias de primer orden. En otras palabras, dadas $N_{d}$ funciones $f_{i}(\mathbf{r}, t), i=1, \ldots, N_{d}$, de las variables de estado y del tiempo, el sistema se expresa

$$
\frac{d r_{i}}{d t}=f_{i}(\mathbf{r}, t), \quad i=1, \ldots, N_{d}
$$

Cuando ninguna de las funciones $f_{i}$ depende explícitamente del tiempo, se dice que el sistema es autónomo.

Para poder obtener la solución al sistema dinámico, que llamaremos trayectoria u órbita, es necesario contar con la información total del estado del sistema en algún instante $t_{0}$. Es 
decir, se debe tener el valor de $\mathbf{r}_{t_{0}}$. En el caso de flujos, la unión del sistema de ecuaciones diferenciales ordinarias de primer orden junto con la condición a un dado tiempo, es llamado problema de valores iniciales (PVI).

El teorema de existencia y unicidad de la solución de un PVI, que puede consultarse en Birkhoff (1927), sólo garantiza dicha solución en un entorno de la condición inicial y para un intervalo de tiempo, ambos suficientemente pequeños.

\subsection{Sistemas dinámicos Hamiltonianos}

Los conceptos básicos de teoría de sistemas Hamiltonianos aquí definidos han sido extraídos mayoritariamente de Ferraz-Mello (2007), Morbidelli (2006) y Arnold (1989).

\subsubsection{Formalismo Hamiltoniano}

Se dice que un sistema de ecuaciones diferenciales ordinarias autónomas es un flujo Hamiltoniano autónomo si se cumplen las siguientes condiciones. La dimensión del vector $\mathbf{r}$ debe ser par; es decir, $N_{d}=2 n_{d}\left(n_{d} \in \mathbb{N}\right)$. Debe existir una función $H\left(p_{1}, \ldots, p_{n_{d}}, q_{1}, \ldots, q_{n_{d}}\right)$ de forma tal que renombrando de alguna manera las $2 n_{d}$ componentes de $\mathbf{r}$ como $q_{1}, \ldots, q_{n_{d}} \mathrm{y}$ $p_{1}, \ldots, p_{n_{d}}$ las Ecs. (1.2) puedan ser reescritas así:

$$
\frac{d p_{i}}{d t}=-\frac{\partial H}{\partial q_{i}} ; \quad \frac{d q_{i}}{d t}=\frac{\partial H}{\partial p_{i}}
$$

para $i=1, \ldots, n_{d}$. La función $H$ es llamada Hamiltoniana del sistema y las variables $q_{1}, \ldots, q_{n_{d}}$ y $p_{1}, \ldots, p_{n_{d}}$ son llamadas coordenadas generalizadas y momentos generalizados, respectivamente. La dimensión $n_{d}$ de los vectores $\mathbf{q} \equiv\left(q_{1}, \ldots, q_{n_{d}}\right)$ y $\mathbf{p} \equiv\left(p_{1}, \ldots, p_{n_{d}}\right)$ es llamada número de grados de libertad. Se dice que el sistema de ecuaciones descripto por Ec. (1.3) está en forma canónica. Se llama mapa simpléctico a un sistema Hamiltoniano de tiempo discreto. El mismo consiste en un mapeo, como el dado por Ec. (1.1), cuya matriz Jacobiana

$$
\mathbf{M} \equiv \mathbf{D f}(\mathbf{r}) \equiv \frac{d}{d \mathbf{r}} \mathbf{f}(\mathbf{r}),
$$

satisface la siguiente condición simpléctica: $\mathbf{M}^{T} \mathbf{J M}=\mathbf{J}$, donde $\mathbf{J}$ posee la siguiente forma:

$$
\mathbf{J} \equiv\left(\begin{array}{cc}
\mathbf{0} & \mathbf{I} \\
-\mathbf{I} & \mathbf{0}
\end{array}\right)
$$

con I y $\mathbf{0}$, respectivamente, la matriz identidad y la matriz nula, ambas de dimensión $n_{d} \times n_{d}$. En esta tesis se utilizará la siguiente convención respecto a la notación de grados de libertad o dimensionalidad de sistemas Hamiltonianos. Un flujo Hamiltoniano con $n_{d}$ grados de libertad $\left(N_{d}\right.$-dimensional), se caracterizará con la notación "Hamiltoniano $n_{d}$ DoF". Por otro lado, un mapa simpléctico $N_{d}$-dimensional se notará como un "mapa $N_{d} \mathrm{D}$ ".

\subsubsection{Movimiento integrable y variables ángulo-acción}

En la sección 1.1 se habló de la existencia y unicidad de la solución de un PVI, pero nada se dijo sobre la posibilidad o no de construir una solución analítica, exacta y válida para todo tiempo (como es el caso del problema de dos cuerpos). Tal propiedad ha motivado la noción 
de integrabilidad. Para sistemas dinámicos generales, es difícil definir el concepto de sistema integrable. Sin embargo, para sistemas Hamiltonianos, se hace uso del teorema de Liouville logrando una definición de integrabilidad útil en las aplicaciones. El teorema de Liouville afirma que si un sistema canónico de $n_{d}$ grados de libertad admite $n_{d}$ integrales

$$
f_{i}(\mathbf{p}, \mathbf{q})=c_{i}=\text { const } \quad\left(i=1, \ldots, n_{d}\right)
$$

que son independientes, cumplen que $\left\{f_{i}, f_{j}\right\}=0$ para todo $i \neq j(\{\cdot, \cdot\}$ denota el corchete de Poisson) y pueden resolverse para los $n_{d}$ momentos $p_{i}$, entonces el sistema es completamente integrable y la solución general puede ser construida por medio de cuadraturas.

Los sistemas Hamiltonianos integrables que se obtendrán, durante el desarrollo de esta tesis, al realizar aproximaciones a sistemas casi-integrables podrán clasificarse en tres tipos. Tal clasificación puede hallarse en Morbidelli (2006) y depende de las variables de las cuales depende explícitamente el Hamiltoniano.

- $H=H\left(p_{1}, \ldots, p_{n_{d}}\right)$; depende solamente de los momentos generalizados. En este caso la solución consiste en que los momentos $p_{i}$ son constantes de movimiento y las coordenadas $q_{i}$ se mueven linealmente con el tiempo, a velocidades constantes $\omega_{i}=\partial H / \partial p_{i}$, para $1 \leq i \leq n_{d}$

- $H=H(p, q)$; depende de sólo un grado de libertad. En este caso el sistema es integrable porque posee una constante de momvimiento, que es el Hamiltoniano mismo. El movimiento evoluciona a lo largo de curvas de nivel de $H$ en el espacio bidimensional $(p, q)$.

- $H=H\left(p_{1}, \ldots, p_{n_{d}}, q_{k}\right)$; depende de sólo una coordenada. En este caso el sistema es integrable porque posee $n_{d}$ constantes de movimiento independientes que son $p_{1}, \ldots, p_{k-1}$, $p_{k+1}, \ldots, p_{n_{d}}$ y $H$. El movimiento preserva los valores de $p_{1}, \ldots, p_{k-1}, p_{k+1}, \ldots, p_{n_{d}}, \mathrm{y}$ evoluciona a lo largo de curvas de nivel de $\mathrm{H}$ sobre el plano $\left(p_{k}, q_{k}\right)$.

Si a las hipótesis del teorema de Liouville se le agrega la condición de que la superficie $n_{d}$-dimensional implicitamente definida por las constantes de movimiento $f_{1}, \ldots, f_{n_{d}}$ sea compacta, se tiene una versión extendida del teorema cuya tesis afirma que es posible introducir nuevos momentos $\boldsymbol{I}$ y coordenadas $\boldsymbol{\theta}$ tales que:

- Las coordenadas $\theta_{1}, \ldots, \theta_{n_{d}}$ son ángulos, definidos cíclicamente en el intervalo $\mathbb{T} \equiv$ $[0,2 \pi)$, y la transformación canónica, $\mathbf{p}(\boldsymbol{I}, \boldsymbol{\theta}), \mathbf{q}(\boldsymbol{I}, \boldsymbol{\theta})$, es $2 \pi$-periódica en los mismos.

- En las nuevas variables, el Hamiltoniano es una función de los nuevos momentos $\boldsymbol{I}$ solamente; es decir $H \equiv H(\boldsymbol{I})$.

Los nuevos momentos son llamados acciones del sistema y el conjunto de variables $(\boldsymbol{\theta}, \boldsymbol{I})$ es llamado variables ángulo-acción.

El conjunto de variables ángulo-acción no es único. De hecho, cualquier transformación lineal del tipo

$$
\boldsymbol{J}=A \boldsymbol{I}, \quad \boldsymbol{\psi}=\left(A^{T}\right)^{-1} \boldsymbol{\theta},
$$

donde $A$ es una matriz con coeficientes enteros y determinante unitario, y $A^{-T} \equiv\left(A^{T}\right)^{-1}$ es la inversa de su traspuesta, define un nuevo conjunto de variables ángulo-acción. Por lo tanto, el número de conjuntos distintos de variables ángulo-acción es infinito numerable. 


\subsubsection{Degeneración, resonancias y no-linealidad}

Teniendo en cuenta lo analizado en la sección 1.2.2 se puede concluir que la dinámica de todo sistema Hamiltoniano independiente de los ángulos, $H(\boldsymbol{I})$, consiste en valores constantes de las acciones $\left(I_{j}=c_{j}=\right.$ const $)$ junto con comportamientos lineales de las variables ángulo $\left(\theta_{j}=\theta_{j}(0)+\omega_{j} t\right)$, para $1 \leq j \leq n_{d}$, donde se han utilizado las frecuencias constantes:

$$
\boldsymbol{\omega} \equiv \boldsymbol{\omega}(\boldsymbol{I})=\frac{\partial H}{\partial \boldsymbol{I}} .
$$

Se define un toro $n$-dimensional a toda variedad que puede ser parametrizada por $n$ ángulos independientes, y se lo denota $\mathbb{T}^{n}$. La variedad $n_{d}$-dimensional formada por aquellos puntos del espacio de fases que comparten un mismo valor del vector de acciones y cuyos ángulos pertenecen al intervalo $[0,2 \pi)$, es un ejemplo de toro $n_{d}$-dimensional.

Si las frecuencias son tales que la ecuación

$$
\mathbf{k} \cdot \boldsymbol{\omega}=\sum_{j=1}^{n_{d}} k_{j} \omega_{j}=0, \quad \mathbf{k} \equiv\left(k_{1}, \ldots, k_{n_{d}}\right) \in \mathbb{Z}^{n_{d}}
$$

admite como única solución entera $\mathbf{k}=(0, \ldots, 0)$, el movimiento cubre densamente al toro $n_{d}$-dimensional. En este caso se dice que las frecuencias son no resonantes y el movimiento es llamado quasi-periódico. En este caso el toro se clasifica como no resonante.

Por otro lado, en el caso en que la Ec. (1.9) admita como solución $n$, con $1 \leq n<n_{d}$, vectores enteros no nulos independientes $\mathbf{k}^{1}, \ldots, \mathbf{k}^{n}$, la trayectoria llenará densamente un toro, $\mathbb{T}^{m}$, de dimensión $m=n_{d}-n$ y se dice que las frecuencias están en una resonancia de multiplicidad $n$. En este caso el toro se clasifica como resonante. Se llama orden de la resonancia a la cantidad

$$
\min _{1 \leq \mathrm{j} \leq \mathrm{n}}\left|\mathbf{k}^{\mathrm{j}}\right|, \quad \text { con } \quad\left|\mathbf{k}^{\mathrm{j}}\right| \equiv\left|\mathrm{k}_{1}^{\mathrm{j}}\right|+\cdots+\left|\mathrm{k}_{\mathrm{n}_{\mathrm{d}}}^{\mathrm{j}}\right| .
$$

Existen varias formas de definir el concepto de degeneración (Ferraz-Mello, 2007). Una de las formas es la caracterización de Schwarzschild, que está basada en el concepto de resonancia. Se dice que una degeneración es esencial cuando existe al menos una relación de conmensurabilidad entre las frecuencias (1.9) que es independiente de las condiciones iniciales.

La otra noción de degeneración fue introducida por Kolmogorov y establece que el Hamiltoniano es no degenerado si y sólo si

$$
\operatorname{det}\left(\frac{\partial \boldsymbol{\omega}}{\partial \boldsymbol{I}}\right)=\operatorname{det}\left(\frac{\partial^{2} H_{0}}{\partial I_{i} \partial I_{j}}\right) \neq 0 \text {. }
$$

Esta condición garantiza la invertibilidad local de la tranformación $\boldsymbol{\omega}(\boldsymbol{I})$ entre acciones y frecuencias.

Existen sistemas que son degenerados según Kolmogorov (K-degenerados), pero no según Schwarzschild, como por ejemplo el Hamiltoniano lineal en las acciones dado por $H=\sqrt{2} I_{1}+$ $\sqrt{5} I_{2}+\pi I_{3}$. Como ejemplos de sistema que son tanto esencialmente degenerados como Kdegenerados se tiene al dado por $H=2 I_{1}+5 I_{2}-1 I_{3}$. Si un sistema es K-degenerado, entonces existe al menos una dirección en el espacio de acciones a lo largo de la cual las frecuencias no cambian. Contrariamente, si el sistema no es K-degenerado, al menos una frecuencia debe cambiar para cualquier desplazamiento arbitrario pequeño en el espacio de 
acciones. En este caso, los toros resonantes son densos en el espacio de fases y las acciones que "etiquetan" a estos toros resonantes, son densas en el espacio de acciones. Sin embargo, para este tipo de sistemas, la medida de los toros resonantes es nula, incluso considerando todas las multiplicidades, mientras que la medida de los toros no resonantes es uno.

Por último, analicemos la estructura del conjunto de acciones y frecuencias que satisfacen una dada condición de resonancia. A tal conjunto se lo llama superficie resonante, independientemente de la dimensionalidad del problema. Trabajando en el espacio de frecuencias se tiene que (1.9) representa una superficie de nivel de una función lineal (hiperplano) cuyo vector normal es $\mathbf{k}$ y que pasa por el origen. En el caso $n_{d}=2$ se trata de una recta mientras que en el caso de $n_{d}=3$ representa un plano.

De (1.8) y (1.9) se obtiene la condición de superficie resonante en el espacio de acciones:

$$
\mathbf{k} \cdot \boldsymbol{\omega}(\boldsymbol{I})=\sum_{j=1}^{n_{d}} k_{j} \omega_{j}(\boldsymbol{I})=0, \quad \mathbf{k} \in \mathbb{Z}^{n_{d}} .
$$

La forma de tales superficies depende de la relación funcional entre las frecuencias y las acciones y por lo tanto, depende del Hamiltoniano no perturbado. Por ejemplo, para $H_{0}(\boldsymbol{I})=$ $I_{1}^{3}+I_{2}^{2}+I_{3}$, se tiene que $\boldsymbol{\omega}(\boldsymbol{I})=\left(3 I_{1}^{2}, 2 I_{2}, 1\right)$ y que la superficie resonante asociada al armónico $\mathbf{k}$ es $3 k_{1} I_{1}^{2}+2 k_{2} I_{2}+k_{3}=0$. La misma representa un cilindro parabólico con generatriz paralela al eje asociado a $I_{3}$.

Tanto en el espacio de acciones como de frecuencias, la superficie resonante es una variedad de dimensión $n_{d}-1$. Su intersección con la superficie de energía constante, $H_{0}(\boldsymbol{I})=h$, que tiene dimensión $n_{d}-1$, da una variedad de dimensión $n_{d}-2$, considerándola como variedad perteneciente al espacio de acciones o frecuencias. Esto implica que para $n_{d}=2$ tal intersección es un punto mientras que para $n_{d}=3$ es una curva.

El considerar las numerablemente infinitas resonancias que existen en un sistema no Kdegenerado, nos dice que para el caso bidimensional habrá una cantidad infinita de puntos (acción o frecuencia) resonantes que serán densos en la superficie de energía. Para el caso tridimensional, se formará una red de superficies resonantes, o resonance web que cubrirá densamente la superficie energética. Este término se utiliza también para casos con $n_{d}>3$.

En este trabajo se dirá que un sistema Hamiltoniano integrable es un sistema lineal si y sólo si dicho Hamiltoniano es una función lineal de las variables acción. Una característica de los sistemas lineales es la isocronicidad, que consiste en el hecho de que el vector de frecuencias resulta constante e independiente del estado del sistema. Se define como resonancia lineal a aquella resonancia en sistemas lineales. Por ejemplo, para $n_{d}=3$ sería $H_{0}(\boldsymbol{I})=a I_{1}+b I_{2}+c I_{3}$, con lo cual $\boldsymbol{\omega}=(a, b, c)$. De (1.11) se tiene que todo sistema lineal es K-degenerado.

Por otro lado, se dirá que un sistema Hamiltoniano integrable es un sistema no lineal cuando la dependencia del Hamiltoniano respecto de las variables acción no sea lineal. Se caracteriza por el hecho de que al menos una de las frecuencias cambia al cambiar el estado del sistema, por lo que posee una dinámica no-isócrona. Una resonancia no lineal es aquella resonancia que tiene lugar en sistemas Hamiltonianos de esta clase.

\subsubsection{Sistemas Hamiltonianos casi-integrables y teoría perturbativa}

Un sistema Hamiltoniano es casi-integrable si es posible elegir un conjunto canónico de variables ángulo-acción de forma tal que su Hamiltoniano pueda ser escrito como

$$
H(\boldsymbol{I}, \boldsymbol{\theta})=H_{0}(\boldsymbol{I})+\epsilon H_{1}(\boldsymbol{I}, \boldsymbol{\theta}),
$$


donde $\epsilon$ es un parámetro pequeño y tanto $H_{1}$ como las derivadas de $H_{0}$ son de $\mathcal{O}(1)>>\mathcal{O}(\epsilon)$. El Hamiltoniano $H_{0}$ es usualmente llamado la aproximación integrable o el Hamiltoniano no perturbado, mientras que $\epsilon H_{1}$ es su perturbación.

Además, para $\epsilon \neq 0$ y pequeño, las varibles acción son en realidad quasi-acciones.

A continuación se presentarán algunos conceptos básicos de la teoría perturbativa (Morbidelli, 2006), que serán utilizados posteriormente en esta tesis. La idea básica de todo enfoque perturbativo para un sistema Hamiltoniano es encontrar una transformación canónica cercana a la identidad de la forma

$$
\boldsymbol{I}=\boldsymbol{I}^{1}+\epsilon \boldsymbol{f}_{1}\left(\boldsymbol{I}^{1}, \boldsymbol{\theta}^{1}\right), \quad \boldsymbol{\theta}=\boldsymbol{\theta}^{1}+\epsilon \boldsymbol{g}_{1}\left(\boldsymbol{I}^{1}, \boldsymbol{\theta}^{1}\right),
$$

de forma tal que al reemplazar (1.14) en (1.13) se obtiene

$$
H^{1}\left(\boldsymbol{I}^{1}, \boldsymbol{\theta}^{1}\right)=H_{0}\left(\boldsymbol{I}^{1}\right)+\epsilon \bar{H}_{1}\left(\boldsymbol{I}^{1}\right)+\epsilon^{2} H_{2}\left(\boldsymbol{I}^{1}, \boldsymbol{\theta}^{1}\right),
$$

donde se han introducido nuevas funciones de orden unidad, $\bar{H}_{1}$ y $H_{2}$. En caso de poderse encontrar esta transformación, se tendrá que $H_{0}+\epsilon \bar{H}_{1}$ será la aproximación integrable de orden $\epsilon^{2}$ de la dinámica real de $H . \boldsymbol{\theta}^{1}$ y $\boldsymbol{I}^{1}$ serán las variables ángulo-acción de dicha aproximación integrable. Puede intentarse iterar este procedimiento, buscando una secuencia de transformaciones canónicas de la forma:

$$
\boldsymbol{I}^{r-1}=\boldsymbol{I}^{r}+\epsilon^{r} \boldsymbol{f}_{r}\left(\boldsymbol{I}^{r}, \boldsymbol{\theta}^{r}\right), \quad \boldsymbol{\theta}^{r-1}=\boldsymbol{\theta}^{r}+\epsilon^{r} \boldsymbol{g}_{r}\left(\boldsymbol{I}^{r}, \boldsymbol{\theta}^{r}\right),
$$

de forma tal que el Hamiltoniano queda:

$$
H^{r}\left(\boldsymbol{I}^{r}, \boldsymbol{\theta}^{r}\right)=H_{0}\left(\boldsymbol{I}^{r}\right)+\epsilon \bar{H}_{1}\left(\boldsymbol{I}^{r}\right)+\cdots+\epsilon^{r} \bar{H}_{r}\left(\boldsymbol{I}^{r}\right)+\epsilon^{r+1} H_{r+1}\left(\boldsymbol{I}^{r}, \boldsymbol{\theta}^{r}\right) .
$$

En este caso las variables $\left(\boldsymbol{I}^{r}, \boldsymbol{\theta}^{r}\right)$, son las variables acción-ángulo de la aproximación integrable a orden $\epsilon^{r+1}$ :

$$
H_{0}\left(\boldsymbol{I}^{r}\right)+\epsilon \bar{H}_{1}\left(\boldsymbol{I}^{r}\right)+\cdots+\epsilon^{r} \bar{H}_{r}\left(\boldsymbol{I}^{r}\right) .
$$

Este proceso iterativo no siempre puede realizarse indefinidamente debido a problemas de convergencia que pueden suceder, y debe detenerse en cierto orden $r$ óptimo.

Uno de los esquemas para encontrar transformaciones que permitan llegar hasta ese orden óptimo es mediante la utilización de series de Lie. Tal enfoque, en su primera iteración, determina que debe cumplirse la llamada ecuación homológica

$$
H_{1}+\left\{H_{0}, \chi\right\}=\bar{H}_{1},
$$

donde las incógnitas son las funciones $\chi\left(\boldsymbol{I}^{1}, \boldsymbol{\theta}^{1}\right)$ y $\bar{H}_{1}\left(\boldsymbol{I}^{1}\right)$. Para resolverlo, se aprovecha el hecho de que el Hamiltoniano $H$ es periódico en $\boldsymbol{\theta}^{1}$, expandiendo $H_{1}$ en series de Fourier como

$$
H_{1}\left(\boldsymbol{I}^{1}, \boldsymbol{\theta}^{1}\right)=\sum_{\mathbf{k} \in \mathbb{Z}^{n_{d}}} c_{\mathbf{k}}\left(\boldsymbol{I}^{1}\right) \exp \left(i \mathbf{k} \cdot \boldsymbol{\theta}^{1}\right)
$$

y proponiendo una solución $\chi$ de forma similar:

$$
\chi\left(\boldsymbol{I}^{1}, \boldsymbol{\theta}^{1}\right)=\sum_{\mathbf{k} \in \mathbb{Z}^{n} d} d_{\mathbf{k}}\left(\boldsymbol{I}^{1}\right) \exp \left(i \mathbf{k} \cdot \boldsymbol{\theta}^{1}\right) .
$$

de forma tal que se cumple que

$$
\left\{H_{0}, \chi\right\}=-i \sum_{\mathbf{k} \in \mathbb{Z}^{n} d} d_{\mathbf{k}}\left(\boldsymbol{I}^{1}\right) \mathbf{k} \cdot \boldsymbol{\omega}_{0}\left(\boldsymbol{I}^{1}\right) \exp \left(i \mathbf{k} \cdot \boldsymbol{\theta}^{1}\right),
$$


donde $\boldsymbol{\omega}_{0} \equiv \nabla_{\boldsymbol{I}^{1}} H_{0}$.

El resultado consiste en que los coeficientes de $\chi$ están relacionados con los de la perturbación $H_{1}$ de la siguiente manera:

$$
d_{\mathbf{0}}=0, \quad d_{\mathbf{k}}\left(\boldsymbol{I}^{1}\right)=-i \frac{c_{\mathbf{k}}\left(\boldsymbol{I}^{1}\right)}{\mathbf{k} \cdot \boldsymbol{\omega}_{0}\left(\boldsymbol{I}^{1}\right)} \quad \forall \mathbf{k} \neq \mathbf{0},
$$

y la función $\bar{H}_{1}$ es

$$
\bar{H}_{1}\left(\boldsymbol{I}^{1}\right)=c_{0}\left(\boldsymbol{I}^{1}\right) .
$$

Observando la Ec. (1.23) se concluye que la función $\chi$ no está definida si simultáneamente se da que existe algún vector $\mathbf{k}$ tal que se cumple la condición resonante, $\mathbf{k} \cdot \boldsymbol{\omega}_{0}\left(\boldsymbol{I}^{1}\right)=0$, para algún valor de $\boldsymbol{I}^{1}$, y que el correspondiente coeficiente de la perturbación, $c_{\mathbf{k}}\left(\boldsymbol{I}^{1}\right)$, es distinto de cero. Esto quiere decir que $\chi$ no puede definirse sobre ninguna nueva acción $\boldsymbol{I}^{1}$ tal que las frecuencias no perturbadas que le corresponden sean conmensurables. Debido a que para todo Hamiltoniano no Kolmogorov-degenerado las resonancias son densas en espacio de acciones, para una perturbación general $H_{1}(1.13)$, no es posible definir a $\chi$ en ningún conjunto abierto del espacio de acciones. Incluso, si dicha perturbación sólo tuviera un número finito de coeficientes no nulos, con cada iteración $r$-ésima del método perturbativo se irían creando armónicos de orden superior en $H_{r+1}\left(\boldsymbol{I}^{r}, \boldsymbol{\theta}^{r}\right)$, de Ec. (1.17).

Esta dificultad inevitable al intentar integrar un sistema Hamiltoniano en cualquier conjunto abierto del espacio de acciones es llamada problema de los pequeños denominadores y es el núcleo de la demostración del teorema de Poincaré sobre la no integrabilidad de sistemas Hamiltonianos casi-integrables genéricos (Poincaré, 1892) Este teorema establece que un sistema Hamiltoniano $H$, bajo ciertas condiciones entre las que se encuentra el no ser K-degenerado, no puede tener una integral $F$ independiente de $H$ expresable formalmente en serie de potencias $\sum_{j \geq 0} F_{j}(\boldsymbol{I}, \boldsymbol{\theta}) \epsilon^{j}$, con coeficientes analíticos (Kozlov, 1983).

\subsubsection{Formas normales}

La forma normal de un Hamiltoniano es una aproximación al mismo. Tal aproximación no necesariamente es integrable pero se espera que facilite el estudio de la dinámica. A continuación se explicarán dos tipos de formas normales que serán necesarios para el desarrollo de esta tesis.

Cuando es posible llevar un Hamiltoniano $H$ a la forma dada por Ec. (1.17) se dice que el Hamiltoniano está en forma normal de Birkhoff a orden $r$ en $\epsilon$. En general, para un sistema Hamiltoniano no Kolmogorov-degenerado, es imposible construir una forma normal de Birkhoff hasta un orden arbitrario en $\epsilon$ sobre un conjunto abierto del espacio de acciones, debido a la densidad de resonancias mencionada en 1.2.3.

Otro tipo de forma normal es la forma normal resonante, que es útil para estudiar la dinámica en entornos de resonancias, reteniendo a bajo orden (en $\epsilon$ ) sólo aquellos términos resonantes esenciales, mientras que los no resonantes son postergados hacia órdenes superiores. A primer orden el Hamiltoniano normal resonante tiene la forma:

$$
H^{1}\left(\boldsymbol{I}^{1}, \boldsymbol{\theta}^{1}\right)=H_{0}\left(\boldsymbol{I}^{1}\right)+\epsilon \bar{H}_{1}^{R}\left(\boldsymbol{I}^{1}, \boldsymbol{\theta}^{1}\right)+\mathcal{O}\left(\epsilon^{2}\right),
$$

donde $H_{1}^{R}$ contiene todos los términos resonantes de $H_{1}$ cuya dinámica nos interesa mantener en la forma normal. Sólo en el caso en que $H_{1}^{R}$ contiene una sola resonancia de multiplicidad uno, también llamada resonancia aislada, la forma normal $H_{0}\left(\boldsymbol{I}^{1}\right)+\epsilon \bar{H}_{1}^{R}\left(\boldsymbol{I}^{1}, \boldsymbol{\theta}^{1}\right)$ es integrable. 
A dicho tipo de forma normal resonante integrable es que pertenece la aproximación del péndulo (ver subsecciones 1.2.7 y 1.4.4).

\subsubsection{La teoría KAM}

Arnold (1989) enuncia el teorema KAM en forma coloquial de la siguiente manera: Si un sistema no perturbado es no degenerado, entonces para perturbaciones Hamiltonianas conservativas suficientemente pequeñas, la mayoría de los toros invariantes no resonantes no se destruyen, sino que son levemente deformados; de forma tal que en el espacio de fases del sistema perturbado también hay toros invariantes que son llenados densamente por trayectorias condicionalmente periódicas, con un número de frecuencias independientes igual al número de grados de libertad.

Estos toros invariantes forman una mayoría en el sentido que la medida del complemento de su unión es pequeño cuando la perturbación es pequeña.

Para entender mejor este enunciado es preciso definir que una frecuencia $\boldsymbol{\omega}$ satisface una condición diofántica si se cumple que:

$$
|\mathbf{k} \cdot \boldsymbol{\omega}(\boldsymbol{I})|>\frac{\gamma}{|\mathbf{k}|^{\tau}}, \quad \forall \mathbf{k} \in \mathbb{Z}^{n_{d}}, \quad \mathbf{k} \neq \mathbf{0},
$$

para algún par de valores positivos de $\gamma$ y $\tau$.

Este teorema supera el problema de los pequeños denominadores (descripto en 1.2.4), a costa de abandonar la idea de encontrar una aproximación integrable en un conjunto abierto. Para su demostración se utiliza una teoría perturbativa de formas normales distinta a las definidas en 1.2.5. El núcleo de tal demostración se centra en probar que para frecuencias que satisfacen la condición diofántica, y para $\epsilon$ suficientemente pequeño, es posible asegurar la convergencia de una serie de transformaciones canónicas en cuyo límite se obtiene un Hamiltoniano con al menos una solución analítica exacta. Tal solución corresponde con la dinámica que transcurre en un toro $n_{d}$-dimensional no resonante. Tales toros son llamados toros invariantes o toros KAM. El teorema asegura que la medida de Lebesgue del conjunto de toros KAM es no nula para $\epsilon$ suficientemente chico. Además, el teorema asegura que al aumentar el tamaño de la perturbación, la cantidad de toros invariantes es reducida y que si $\epsilon$ es suficientemente grande, todos los toros KAM son destruidos.

Esta destrucción de toros KAM en un sistema casi-integrable trae como consecuencia la aparición de nuevos objetos dinámicos, como son los cantori, y las estructuras con órbitas caóticas (ver capítulo 2). Las propiedades de los mismos, incluyendo su topología y la dinámica de las órbitas que incluyen es un tema abierto en la actualidad.

En general todo sistema Hamiltoniano no integrable tendrá órbitas caóticas. Se dice que un sistema presenta espacio de fases dividido si posee simultáneamente la presencia de orbitas regulares y caóticas. Un sistema Hamiltoniano casi-integrable presenta espacio de fases dividido si la medida tanto de toros KAM como de órbitas caóticas no es despreciable.

\subsubsection{El péndulo simple}

En esta sección se presenta el sistema Hamiltoniano del péndulo simple. En 1.4.4 se demostrará analíticamente que dicho sistema constituye un modelo de resonancia no-lineal, mientras que en 4.3.1 se revisa experimentalmente, en una resonancia particular de un sistema Hamiltoniano $3 \mathrm{DoF}$, la validez de tal modelo. 
El péndulo simple consiste en una masa puntual $m$ sometida a una fuerza homogénea y constante $\boldsymbol{F}$ que debe cumplir con la condición de estar a una distancia $l$ constante respecto a un punto inercial fijo (origen). La característica unidimensional le agrega la condición de que el movimiento está contendio en un plano fijo respecto de algún sistema inercial. Sea $\psi$ el ángulo subtendido entre la dirección de la fuerza $\boldsymbol{F}$ y el radio vector que va desde el origen hasta la masa. Sea además, $p$ su variable conjugada, $M=m l^{2}$ su momento de inercia y $U_{0}$ la energía potencial en el punto de equilibrio inestable. Entonces, el Hamiltoniano del péndulo simple es el siguiente:

$$
H(p, \psi)=\frac{1}{2 M} p^{2}-U_{0} \cos \psi
$$

La deducción de la solución del sistema de ecuaciones inducidas por este Hamiltoniano puede consultarse en Chirikov (1979), Ferraz-Mello (2007) y Mestre (2006). De acuerdo al valor de la energía total $h$ existen distintos comportamientos dinámicos. Para $h<U_{0}$ la solución del sistema es

$$
\psi(t)=4 \sum_{n=1}^{\infty} \frac{1}{(2 n-1) \cosh \left((n-1 / 2) \pi \frac{K\left(k^{\prime}\right)}{K(k)}\right)} \sin \left(\omega_{n}(k) t\right),
$$

donde $K(k)$ denota la integral elíptica completa de primera especie:

$$
\begin{gathered}
K(k) \equiv \int_{0}^{\pi / 2} \frac{d \alpha}{\sqrt{1-k^{2} \sin ^{2} \alpha}}, \\
\omega_{0} \equiv \sqrt{U_{0} / M}
\end{gathered}
$$

es la frecuencia de pequeñas oscilaciones, $y$

$$
\begin{aligned}
k & \equiv \sqrt{\frac{h+U_{0}}{2 U_{0}}}, \\
k^{\prime} & \equiv \sqrt{1-k^{2}}, \\
\omega_{n}(k) & \equiv(2 n-1) \frac{\pi \omega_{0}}{2 K(k)} .
\end{aligned}
$$

De (1.28) se puede ver que el ángulo $\psi$ es una función periódica del tiempo, con frecuencia $\omega(k)=\frac{\pi \omega_{0}}{2 K(k)}$, que depende de la energía del péndulo a través de $k$. A esta dinámica se le llama régimen de oscilaciones o libraciones.

Para $h>U_{0}$ el desarrollo de Fourier del ángulo esta dado por:

$$
\pm \psi(t)=2 \omega(k) t+2 \sum_{n=1}^{\infty} \frac{1}{n \cosh \left(n \pi \frac{K\left(k^{\prime}\right)}{K(k)}\right)} \sin \left(\omega_{n}(k) t\right),
$$

donde las nuevas definiciones, respecto de (1.31) son:

$$
\begin{aligned}
k & \equiv \sqrt{\frac{2 U_{0}}{h+U_{0}}} \\
\omega(k) & \equiv \frac{\pi \omega_{0}}{2 k K(k)}, \\
\omega_{n}(k) & \equiv 2 n \omega(k) .
\end{aligned}
$$


Este es el régimen de rotación ya que $\psi$ presenta una evolución secular, dando vueltas completas al origen con una frecuencia igual a $2 \omega(k)$.

Finalmente, para $h=U_{0}$ se tiene una solución en términos de funciones bien conocidas:

$$
\begin{aligned}
& \psi_{s}(t)=4 \arctan \left(e^{\omega_{0} t}\right)-\pi, \\
& p_{s}(t)= \pm 2 \sqrt{M U_{0}} \cos \left(\psi_{s}(t) / 2\right) .
\end{aligned}
$$

Esta curva es llamada separatriz pues divide al espacio de fases en dos regímenes dinámicos diferentes. Cuando $t \rightarrow \infty, \psi_{s} \rightarrow \pi$ y $t \rightarrow-\infty, \psi_{s} \rightarrow-\pi$. Los puntos del espacio de fases de la forma $(p, \psi)=(0, m \pi)$, con $m \in \mathbb{Z}$ son normalmente llamados puntos inestables abigotados, debido a que en los mismos se cruzan transversalmente la variedad estable y la inestable de la separatriz.

Las trayectorias con condiciones iniciales en un entorno de la separatriz son las más sensibles ante las perturbaciones al péndulo, como se verá en la siguiente sección.

\subsubsection{La capa estocástica}

La existencia de separatriz no es una característica única del péndulo simple. Existen diversos sistemas Hamiltonianos integrables que poseen una o más de las mismas, separando trayectorias topológicamente distintas. La existencia de una separatriz en la parte no perturbada de sistemas casi-integrables tiene consecuencias en la estabilidad de órbitas cercanas a la misma por la siguiente razón cualitativa: para toda perturbación oscilatoria, por más leve que sea, existen condiciones iniciales suficientemente cercanas a la separatriz de forma tal que sus correspondientes órbitas alternan "casi aleatoriamente", a lo largo del tiempo, entre ambos regímenes de movimiento, y experimentos numéricos han demostrado que tales órbitas son caóticas. La alta complejidad de la dinámica de estas trayectorias fue mencionada en Poincaré (1892) y posteriormente, fue estudiada en detalle por Mel'nikov (1963) y otros. La región del espacio de fases ocupada por estas trayectorias caóticas consiste en una capa alrededor de la separatriz original, donde la dinámica adquiere características parecidas a las de un proceso aleatorio o estocástico (Zaslavski y Chirikov, 1972). Por tal motivo, se dice que la separatriz se transforma en una capa estocástica, ante la más mínima perturbación. Soskin y Mannella (2009) hacen una revisión de algunas teorías que intentan computar el máximo absoluto del ancho de la capa estocástica y, a su vez, proponen un método cuya predicción concuerda con los experimentos.

Incluso cuando el sistema no perturbado carece de separatriz, la parte resonante de una perturbación genérica induce una forma normal resonante que sí posee una separatriz al tiempo que la parte no resonante de la perturbación es la encargada de perturbarla, reemplazándola por una capa estocástica (Soskin et al., 2009). Por esta razón algunos autores consideran que la perturbación de separatrices es el germen del caos Hamiltoniano.

\subsection{Difusión en el espacio de acciones}

\subsubsection{Coeficientes de difusión}

En general, dado un ensamble de partículas, el coeficiente de difusión, $\mathcal{D}$, asociado a la variable escalar acción, $I$, durante el intervalo temporal $\left[t_{0}, t\right]$, se define a partir del desplazamiento cuadrático medio y el tamaño del intervalo de tiempo (Einstein 1956, Uhlenbeck y 
Ornstein 1930, Klafter et al. 1996):

$$
\mathcal{D} \equiv \frac{\left\langle\left(I(t)-I\left(t_{0}\right)\right)^{2}\right\rangle}{2\left(t-t_{0}\right)}
$$

donde $\langle\cdot\rangle$ denota el promedio sobre las partículas del ensamble. Para un ensamble conformado por $N_{p}$ partículas, fijando $t_{0}=0$ y llamando $I(t, i)$ a la acción de la partícula $i$-ésima al tiempo $t$, se define el desplazamiento cuadrático medio $\left(\gamma^{2}\right)$ como

$$
\gamma^{2}(t) \equiv\left\langle(I(t)-I(0))^{2}\right\rangle \equiv \frac{1}{N_{p}} \sum_{i=1}^{N_{p}}(I(t, i)-I(0, i))^{2},
$$

Entonces, la expresión del coeficiente de difusión (1.35) puede abreviarse así:

$$
\mathcal{D}_{\gamma} \equiv \frac{\gamma^{2}(t)}{2 t} .
$$

Debe aclararse que para que la expresión del coeficiente de difusión sea independiente del tiempo, el desplazamiento cuadrático medio debe ser una función lineal del mismo. Asimismo, si queremos que esta relación lineal sea válida para todo tiempo, es necesario que el coeficiente de difusión tampoco dependa de la acción. Esto se debe a que si hubiese una dependencia con la acción, el efecto neto sobre un ensamble que explora distintas regiones, sería producir un comportamiento del desplazamiento cuadrático medio no lineal. Sin embargo, a menos que se esté tratando de computar la dependencia temporal del coeficiente de difusión, estas dos restricciones son generalmente dejadas de lado por la siguiente razón. Es posible obtener comportamientos localmente lineales del desplazamiento cuadrático medio, acotando el tiempo de evolución de forma tal que el ensamble no se alcance a alejar lo suficiente como para llegar a regiones con coeficientes de difusión sensiblemente distintos a aquel correspondiente a las acciones iniciales del ensamble. De esta forma, es posible asignar un coeficiente de difusión al entorno de una dada acción inicial, enfoque que ha sido adoptado en varios trabajos (Novaković et al. 2010, Todorović et al. 2008, Lega et al. 2003, Froeschlé et al. 2005, Bazzani and Mais 1998, Bazzani et al. 1997, Siboni et al. 1994, Bountis and Kollmann 1994), entre otros).

Para que el coeficiente de difusión sea independiente del tiempo, el desplazamiento cuadrático medio debe ser una función lineal del mismo. En general, a medida que las partículas del ensamble se alejan de su entorno inicial, atraviesan regiones con diferentes propiedades difusivas, de forma tal que el comportamiento global de $\gamma^{2}(t)$ no es lineal. Sin embargo, es posible obtener comportamientos localmente lineales acotando adecuadamente el tiempo de evolución del ensamble. De esta forma, es posible asignar un coeficiente de difusión al entorno de una dada acción inicial, enfoque que ha sido adoptado en varios trabajos (Novaković et al. 2010, Todorović et al. 2008, Lega et al. 2003, Froeschlé et al. 2005, Bazzani and Mais 1998, Bazzani et al. 1997, Siboni et al. 1994, Bountis and Kollmann 1994, entre otros).

De la definición (1.36) se aprecia que la cantidad $\gamma(t) \equiv \sqrt{\gamma^{2}(t)}$ es una estimación del tamaño de la región recorrida por el ensamble durante el tiempo $t$.

Existe otra cantidad estadística que da información sobre el tamaño de la región ocupada por el ensamble al tiempo $t$. Se trata de $\sigma$, la desviación estándar, que definiremos a continuación. Sea $\mu(t)$ la media, o momento de primer orden, de los valores de las acciones del ensamble al tiempo $t$ :

$$
\mu(t) \equiv\langle I(t)\rangle \equiv \frac{1}{N_{p}} \sum_{i=1}^{N_{p}} I(t, i),
$$


se define la varianza del ensamble, $\sigma^{2}$, como el siguiente promedio:

$$
\sigma^{2}(t) \equiv\left\langle(I(t)-\mu(t))^{2}\right\rangle \equiv \frac{1}{N_{p}} \sum_{i=1}^{N_{p}}(I(t, i)-\mu(t))^{2} .
$$

La desviación estándar es la raíz cuadrada de la varianza. Estas dos cantidades son sensibles al grado de difusión de un ensamble respecto al centro geométrico del ensamble y son independientes del desplazamiento del centro geométrico del ensamble $(\mu)$. Por otro lado, tanto $\gamma$ como $\gamma^{2}$, sí son influenciadas por $\mu$. El hecho de que $\gamma(t)$ crezca puede no sólo deberse a que el ensamble se difundió, o que se dispersó, si no que también puede significar que el ensamble se mantuvo unido pero su centro geométrico se trasladó.

Es posible definir un coeficiente de difusión distinto al dado por Ec. (1.35), que esté basado en el cómputo de la varianza:

$$
\mathcal{D}_{\sigma} \equiv \frac{\sigma^{2}(t)-\sigma^{2}\left(t_{0}\right)}{2\left(t-t_{0}\right)} .
$$

Notemos que para el caso en que el tiempo inicial es $t_{0}=0$, y para una varianza inicial despreciable respecto a la varianza en tiempo $t$, este coeficiente de difusión queda:

$$
\mathcal{D}_{\sigma} \approx \frac{\sigma^{2}(t)}{2 t},
$$

teniendo una similaridad con la expresión (1.37).

El hecho de que pueda computarse un coeficiente de difusión utilizando $\gamma^{2}$ y otro utilizando $\sigma^{2}$, es un ejemplo más de que no existe una única manera de definir y de medir la difusión de las acciones no perturbadas. Ello se debe a que hay diferentes enfoques conceptuales que intentan describir los procesos que suceden en sistemas Hamiltonianos no integrables. Uno de estos enfoques consiste en encontrar una ley (en general una ecuación diferencial parcial) que gobierne la evolución de la distribución de densidad de probabilidad de la acción, $\rho(I, t)$. Puede interpretarse que $\rho(I, t) \Delta I$ es la probabilidad de que al tiempo $t$ la partícula de prueba posea un valor $I$ en el intervalo $[I, I+\Delta I]$. También puede interpretarse que $\rho(I, t) N_{p} \Delta I$ es el número de partículas que se hallan en dicho intervalo al tiempo $t$. En la sección 1.3.2 se definen algunos conceptos y métodos de dicha teoría.

Otra aproximación al problema consiste en medir las dependencias con el tiempo de ciertas cantidades estadísticas o promedio, como son $\gamma$ o $\sigma$. Aquí no se busca una ley macroscópica para la distribución densidad de probabilidad, si no que simplemente se busca encontrar una regla general para la evolución de la cantidad estadística con el tiempo. La noción de coeficiente de difusión $\mathcal{D}_{\gamma}$ y $\mathcal{D}_{\sigma}$ corresponde a este enfoque, para dependencias lineales con el tiempo. Dentro de este enfoque, existen variantes producto de la necesidad de especificar los conceptos bajo estudio. No es suficiente con dar la función Hamiltoniana y la energía del sistema. Se debe especificar si las cantidades a medir, llamadas observables, son funciones de un ensamble de trayectorias, son funciones de una única trayectoria, o son funciones de algún subconjunto del espacio de fases.

Las cantidades estadísticas arriba definidas pertenecen al primer caso. Por ejemplo, el observable $\sigma^{2}(t)$ (1.39) es una función cuyos argumentos son los $N_{p}$ valores de las acciones correspondientes a las $N_{p}$ partículas del ensamble, para un dado tiempo fijo $t$. La dependencia temporal de $\sigma^{2}(t)$ es a través de sus argumentos, las acciones. Este tipo de promedios serán llamados promedios de ensamble. 
Para ejemplificar el segundo caso, pensemos que tenemos una sola trayectoria $I(t)$ que evoluciona en el intervalo temporal $[0, t]$, y computamos las siguientes cantidades, que llamaremos respectivamente media de trayectoria única (o media temporal), varianza de trayectoria única y desplazamiento medio cuadrático de trayectoria única :

$$
\begin{aligned}
\mu_{u}(t) & \equiv \bar{I}(t) \quad \equiv \frac{1}{t} \int_{0}^{t} I(\tau) d \tau, \\
\sigma_{u}^{2}(t) & \equiv \overline{\left(I-\mu_{u}\right)^{2}}(t) \equiv \frac{1}{t} \int_{0}^{t}\left(I(\tau)-\mu_{u}(\tau)\right)^{2} d \tau, \\
\gamma_{u}^{2}(t) & \equiv \overline{(I-I(0))^{2}}(t) \equiv \frac{1}{t} \int_{0}^{t}(I(\tau)-I(0))^{2} d \tau,
\end{aligned}
$$

donde - denota el promedio temporal. Aquí la estadística es sobre diferentes tiempos, para una única condición inicial.

Así definidas, estas tres cantidades, son funciones de todos los valores de la acción de una única trayectoria, mientras el tiempo transcurrió entre $0 \mathrm{y} t$. En situaciones en que no se dispone de los valores de la trayectoria para todo un intervalo continuo sino que se posee una serie temporal: $\left\{I\left(t_{i}\right) ; i=0,1, \ldots, n ; n \in \mathbb{N}\right\}$, las Ecs. (1.42) son reemplazadas por

$$
\begin{aligned}
\mu_{u}\left(t_{n}\right) & \equiv \frac{1}{n+1} \sum_{i=0}^{n} I\left(t_{i}\right), \\
\sigma_{u}^{2}\left(t_{n}\right) & \equiv \frac{1}{n+1} \sum_{i=0}^{n}\left(I\left(t_{i}\right)-\mu_{u}\left(t_{i}\right)\right)^{2}, \\
\gamma_{u}^{2}\left(t_{n}\right) & \equiv \frac{1}{n+1} \sum_{i=0}^{n}\left(I\left(t_{i}\right)-I\left(t_{0}\right)\right)^{2}
\end{aligned}
$$

Estas expresiones también se utilizan en el caso de sistemas dinámicos con tiempo discreto, con el correspondiente reemplazo $t_{i}=i$.

De forma análoga a los coeficientes de difusión definidos a través de promedios de ensamble (1.35) y (1.37), es posible definir coeficientes de difusión utilizando promedios temporales (usando $t_{0}=0$ ):

$$
\begin{aligned}
\mathcal{D}_{\gamma_{u}} & \equiv \frac{\gamma_{u}^{2}(t)}{2 t}, \\
\mathcal{D}_{\sigma_{u}} & \equiv \frac{\sigma_{u}^{2}(t)-\sigma_{u}^{2}(0)}{2 t} .
\end{aligned}
$$

Chirikov (1979), Cincotta (2002) y Bountis and Kollmann (1994) han realizado estudios de difusión de trayectoria única, pero utilizando algoritmos más elaborados de promediado temporal, con la intención de disminuir oscilaciones y de descartar que el proceso no sea difusivo. La relación entre coeficientes de difusión con origen en promedios de ensamble, y aquellos con origen en promedios temporales es actualmente un tema de investigación (Bauer et al. 2011, Zaburdaev et al. 2011, Rebenshtok and Barkai 2008, etc.). En general, ambos tipos de promedio dan resultados distintos en sistemas con espacio de fases dividido.

El tercer caso corresponde a computar promedios espaciales en una dada región o variedad del espacio de fases, utilizando una dada medida ${ }^{1} \lambda$. A pesar de que en esta tesis no se trabajará con esta clase de promedios, se dará su definición para un sistema de un grado de libertad.

\footnotetext{
${ }^{1}$ Para una introducción a la teoría de la medida se puede consultar Bartle (1995).
} 
Dado un subconjunto, $\mathcal{A}$, del espacio (plano) de fases del sistema, el promedio de una función $f(I, \theta)$ sobre dicho conjunto, utilizando la medida $\lambda$, se computa a través de la siguiente integral:

$$
\frac{1}{\lambda(\mathcal{A})} \int_{\mathcal{A}} f(I, \theta) \lambda(d I d \theta)
$$

Dado un sistema Hamiltoniano descripto por las variables $(I, \theta) \in \mathbb{R} \times \mathbb{T}^{1}$, sea $M^{t}(t>0)$ un operador evolución en el espacio de acciones tal que $(I(t), \theta(t))=M^{t}(I(0), \theta(0))$ y sea $M^{-t}$ su operador inverso. Si la medida $\lambda$ satisface que para todo subconjunto $\mathcal{A}_{*}$ de $\mathcal{A}$, vale la igualdad $\lambda\left(M^{-t} \mathcal{A}_{*}\right)=\lambda\left(\mathcal{A}_{*}\right)$, entonces la medida es llamada invariante en $\mathcal{A}$. Si además, no es posible descomponer a $\mathcal{A}$ en porciones en las cuales $\lambda$ sea a su vez invariante, se dice que $\lambda$ es ergódica en $\mathcal{A}$ (Eckmann y Ruelle, 1985).

Uno de los corolarios del teorema ergódico (Halmos, 1956) consiste en la siguiente relación entre promedios de trayectoria única asintóticos y promedios espaciales, para una función continua $f$ y una medida ergódica y finita $\lambda$ :

$$
\lim _{t \rightarrow \infty} \frac{1}{t} \int_{0}^{t} f(I(t), \theta(t)) d t=\frac{1}{\lambda(\mathcal{A})} \int_{\mathcal{A}} f(I, \theta) \lambda(d I, d \theta) .
$$

No es eficiente intentar computar una varianza utilizando el promedio espacial para un sistema genérico. Esto se debe a que no se conoce, a priori, el conjunto invariante que contiene a la condición inicial. Además, en los casos de órbitas caóticas, la medida ergódica es imposible de predecir debido a que dicho conjunto invariante presenta una topología muy compleja.

En esta tesis se trabajará solamente con cantidades estadísticas de ensamble, al igual que en Cordeiro y Mendes de Souza (2005) y en Cordeiro (2006). Fundamentalmente, se aplicará una generalización vectorial de la varianza (1.39), que a continuación se define. Sea $\boldsymbol{I}$ un vector con componentes $I_{j}\left(j=1, \ldots, n_{d}\right)$, y sea $I_{j}(t, i)$ el valor de la componente $j$-ésima, de dicho vector, asociada a la partícula $i$-ésima. Entonces, la media del ensamble asociada a esta componente es:

$$
\mu_{j}(t) \equiv\left\langle I_{j}(t)\right\rangle \equiv \frac{1}{N_{p}} \sum_{i=1}^{N_{p}} I_{j}(t, i),
$$

y la correspondiente varianza del ensamble está dada por:

$$
\sigma_{j}^{2}(t) \equiv\left\langle\left(I_{j}(t)-\mu_{j}(t)\right)^{2}\right\rangle \equiv \frac{1}{N_{p}} \sum_{i=1}^{N_{p}}\left(I_{j}(t, i)-\mu_{j}(t)\right)^{2} .
$$

La generalización del desplazamiento cuadrático medio prosigue de forma similar:

$$
\gamma_{j}^{2}(t) \equiv\left\langle\left(I_{j}(t)-I_{j}(0)\right)^{2}\right\rangle \equiv \frac{1}{N_{p}} \sum_{i=1}^{N_{p}}\left(I_{j}(t, i)-I_{j}(0, i)\right)^{2},
$$

\subsubsection{Difusión y transporte}

No existe una definición universal del término difusión. La misma depende del área de investigación, del sistema bajo estudio, de las variables adoptadas y de las herramientas matemáticas utilizadas. Se darán algunas nociones de dicho concepto en el marco de la teoría de procesos estocásticos y en el marco de sistemas Hamiltonianos. 
Previamente se darán unas definiciones y propiedades básicas de la teoría de procesos estocásticos, que han sido extraídas de los libros de Gardiner (2004) y de Arnold (1973).

Un proceso estocástico o aleatorio, también llamado sistema estocástico, es un sistema tal que en cada tiempo $t$ su estado depende, parcial o totalmente, del resultado de uno o más eventos aleatorios. Al igual que en un sistema dinámico (sección 1.1), los estados estarán determinados por los números reales que puede tomar un cierto conjunto de variables (llamadas variables aleatorias), conformando una variedad llamada espacio de fases. Dado un espacio de fases con dimensión $N_{d}$, se notará a las variables aleatorias como $X_{j}(t),(j=$ $\left.1, \ldots, N_{d}\right)$. Las mismas conforman las componentes de un vector aleatorio, $\boldsymbol{X}(t)$.

No debe confundirse a las variables o vectores aleatorios recién definidos con los correspondientes valores que se miden. Dado un tiempo $t$ fijo, es posible medir los valores de las variables $x_{j}(t),\left(i=1, \ldots, N_{d}\right)$, y asignarlas como componentes del vector aleatorio $\mathbf{x}(t)$. Tales mediciones conforman el estado al tiempo $t$ de (sólo) una realización del proceso estocástico, también llamada trayectoria muestra. Similarmente, dada una sucesión de tiempos $t_{1}, t_{2}, \ldots$ es posible medir una sucesión de cantidades vectoriales $\mathbf{x}\left(t_{1}\right), \mathbf{x}\left(t_{2}\right), \ldots$

Sin entrar en la teoría de la medida, necesaria para definir rigurosamente los conceptos estocásticos, se dirá que el conjunto de todas las posibles realizaciones del proceso estocástico constituye un espacio abstracto usualmente llamado espacio de muestras (sample space), simbolizado $\mathcal{M}$. A su vez, se le llama espacio de eventos, denotado $\mathfrak{E}$, al conjunto constituido por todos aquellos subconjuntos del espacio de muestras a los que se les puede asignar una probabilidad de ocurrencia, simbolizada $\mathcal{P}$.

Se asume que para toda sucesión de tiempos $\left\{t_{k}\right\}_{k \in \mathbb{N}}$ existe la densidad de probabilidad conjunta que es una función de la forma:

$$
\begin{aligned}
\rho & \equiv \rho\left(\mathbf{x}\left(t_{1}\right) ; t_{1} ; \mathbf{x}\left(t_{2}\right) ; t_{2} ; \ldots\right) \\
& =\rho\left(x_{1}\left(t_{1}\right), x_{2}\left(t_{1}\right), \ldots x_{N_{d}}\left(t_{1}\right) ; t_{1} ; \ldots, x_{1}\left(t_{k}\right), x_{2}\left(t_{k}\right), \ldots x_{N_{d}}\left(t_{k}\right) ; t_{k} ; \ldots\right)
\end{aligned}
$$

Estas funciones describen al sistema estocástico completamente. La densidad de probabilidad conjunta debe interpretarse de la siguiente manera. Sea el espacio de eventos (E) el espacio de dimensión infinita formado por el producto directo de los espacios de eventos unidimensionales asociados a cada una de las componentes de cada uno de los vectores aleatorios $\left(\boldsymbol{X}\left(t_{k}\right)\right)$ asociados a cada tiempo $\left(t_{k}\right)$. Sea $\mathcal{A} \in \mathfrak{E}$ el conjunto (con forma de hipercubo y tamaño diferencial) definido por todos los eventos, $\left(\mathbf{y}\left(t_{1}\right), \mathbf{y}\left(t_{2}\right), \ldots\right)$, que satisfacen simultánemamente que $x_{j}\left(t_{k}\right)<y_{j}\left(t_{k}\right)<x_{j}\left(t_{k}\right)+d x_{j}$ para todo $j=1, \ldots, N_{d}$ y para todo $k \in \mathbb{N}$. Entonces, la información contenida en la densidad de probabilidad se relaciona con la probabilidad de que una realización del proceso estocástico $\boldsymbol{X}(t)$ pertenezca a $\mathcal{A}$ de la siguiente manera:

$$
\begin{aligned}
\mathcal{P}(\mathcal{A}) \equiv & \rho\left(x_{1}\left(t_{1}\right), x_{2}\left(t_{1}\right), \ldots x_{N_{d}}\left(t_{1}\right) ; t_{1} ; \ldots, x_{1}\left(t_{k}\right), x_{2}\left(t_{k}\right), \ldots x_{N_{d}}\left(t_{k}\right) ; t_{k} ; \ldots\right) \\
& \times d x_{1}\left(t_{1}\right) d x_{2}\left(t_{1}\right) \ldots d x_{1}\left(t_{k}\right) d x_{2}\left(t_{k}\right) \ldots
\end{aligned}
$$

A la tríada conformada por $\mathcal{M}, \mathfrak{E}$ y $\mathcal{P}$ se le suele llamar espacio de probabilidad.

A continuación utilizaremos la notación abreviada para vectores: $\mathbf{x}_{k} \equiv \mathbf{x}\left(t_{k}\right)$. La probabilidad de que habiendo ocurrido el evento $\left(\mathbf{y}_{1}, \mathbf{y}_{2}, \ldots\right)$ suceda el evento $\left(\mathbf{x}_{1}, \mathbf{x}_{2}, \ldots\right)$ es llamada densidad de probabilidad condicional y se computa en términos de densidades de probabilidad conjuntas así:

$$
\rho\left(\mathbf{x}_{1}, t_{1} ; \mathbf{x}_{2}, t_{2} ; \ldots \mid \mathbf{y}_{1}, \tau_{1} ; \mathbf{y}_{2}, \tau_{2} ; \ldots\right)=\frac{\rho\left(\mathbf{x}_{1} ; t_{1} ; \mathbf{x}_{2} ; t_{2} ; \ldots, \mathbf{y}_{1} ; \tau_{1} ; \mathbf{y}_{2} ; \tau_{2} ; \ldots\right)}{\rho\left(\mathbf{y}_{1} ; \tau_{1} ; \mathbf{y}_{2} ; \tau_{2} ; \ldots\right)}
$$


Estas definiciones son válidas independientemente del orden de los tiempos, pero en lo que sigue se considerará que los tiempos están ordenados de la forma

$$
t_{1} \geq t_{2} \geq \ldots \tau_{1} \geq \tau_{2} \geq \ldots
$$

Un proceso Markoviano es un proceso estocástico en el cual la densidad de probabilidad condicional queda completamente determinada por la más reciente condición:

$$
\rho\left(\mathbf{x}_{1}, t_{1} ; \mathbf{x}_{2}, t_{2} ; \ldots \mid \mathbf{y}_{1}, \tau_{1} ; \mathbf{y}_{2}, \tau_{2} ; \ldots\right)=\rho\left(\mathbf{x}_{1}, t_{1} ; \mathbf{x}_{2}, t_{2} ; \ldots \mid \mathbf{y}_{1}, \tau_{1}\right) .
$$

La propiedad Markoviana permite definir todo en términos de densidades de probabilidad simples $\rho\left(\mathbf{x}_{1}, t_{1} \mid \mathbf{y}_{1}, \tau_{1}\right)$, y expresar la ecuación de Chapman-Kolmogorov (C-K):

$$
\rho\left(\mathbf{x}_{1}, t_{1} \mid \mathbf{x}_{3}, t_{3}\right)=\int \mathbf{d x}_{2} \rho\left(\mathbf{x}_{1}, t_{1} \mid \mathbf{x}_{2}, t_{2}\right) \rho\left(\mathbf{x}_{2}, t_{2} \mid \mathbf{x}_{3}, t_{3}\right)
$$

Esta ecuación funcional es no lineal y relaciona entre sí a las densidades de probabilidad condicionales $\rho\left(\mathbf{x}_{k}, t_{k} \mid \mathbf{x}_{k^{\prime}}, t_{k^{\prime}}\right)$, con $k, k^{\prime} \in \mathbb{N}$. La misma puede llevarse a una versión diferencial pidiendo que $\rho$ sea diferenciable y que existan los siguientes límites, para todo $\epsilon>0$ :

$$
\lim _{\Delta t \rightarrow 0} \frac{\rho(\mathbf{x}, t+\Delta t \mid \mathbf{z}, t)}{\Delta t}=W(\mathbf{x} \mid \mathbf{z}, t),
$$

uniformemente en $\mathbf{x}, \mathbf{z}$ y $t$ para $|\mathbf{x}-\mathbf{z}| \geq \epsilon$;

$$
\begin{aligned}
& \lim _{\Delta t \rightarrow 0} \frac{1}{\Delta t} \int_{|\mathbf{x}-\mathbf{z}|<\epsilon} d \mathbf{x}\left(x_{j}-z_{j}\right) \rho(\mathbf{x}, t+\Delta t \mid \mathbf{z}, t)=A_{j}(\mathbf{z}, t)+\mathcal{O}(\epsilon), \\
& \lim _{\Delta t \rightarrow 0} \frac{1}{\Delta t} \int_{|\mathbf{x}-\mathbf{z}|<\epsilon} d \mathbf{x}\left(x_{j}-z_{j}\right)\left(x_{j^{\prime}}-z_{j^{\prime}}\right) \rho(\mathbf{x}, t+\Delta t \mid \mathbf{z}, t)=B_{j j^{\prime}}(\mathbf{z}, t)+\mathcal{O}(\epsilon),
\end{aligned}
$$

uniformemente en $\mathbf{z}, \epsilon \mathrm{y} t$.

Bajo estas hipótesis, la ecuación integral de C-K (1.56) deviene en la ecuación diferencial de C-K (Gardiner, 2004):

$$
\begin{aligned}
\frac{\partial \rho}{\partial t}\left(\mathbf{z}, t \mid \mathbf{y}, t^{\prime}\right)= & -\sum_{j=1}^{N_{d}} \frac{\partial}{\partial z_{j}}\left[A_{j}(\mathbf{z}, t) \rho\left(\mathbf{z}, t \mid \mathbf{y}, t^{\prime}\right)\right] \\
& +\sum_{j, j^{\prime}} \frac{1}{2} \frac{\partial^{2}}{\partial z_{j} \partial z_{j^{\prime}}}\left[B_{j j^{\prime}}(\mathbf{z}, t) \rho\left(\mathbf{z}, t \mid \mathbf{y}, t^{\prime}\right)\right] \\
& +\int d \mathbf{x}\left[W(\mathbf{z} \mid \mathbf{x}, t) \rho\left(\mathbf{x}, t \mid \mathbf{y}, t^{\prime}\right)-W(\mathbf{x} \mid \mathbf{z}, t) \rho\left(\mathbf{z}, t \mid \mathbf{y}, t^{\prime}\right)\right] .
\end{aligned}
$$

A continuación analicemos tres procesos ideales que son solución de la ecuación diferencial de C-K.

Si pedimos que $W(\mathbf{z} \mid \mathbf{x}, t)$ sea nulo, la Ec. (1.59) se reduce a la Ecuación de Fokker-Planck:

$$
\begin{aligned}
\frac{\partial \rho}{\partial t}\left(\mathbf{z}, t \mid \mathbf{y}, t^{\prime}\right)= & -\sum_{j=1}^{N_{d}} \frac{\partial}{\partial z_{j}}\left[A_{j}(\mathbf{z}, t) \rho\left(\mathbf{z}, t \mid \mathbf{y}, t^{\prime}\right)\right] \\
& +\sum_{j, j^{\prime}} \frac{1}{2} \frac{\partial^{2}}{\partial z_{j} \partial z_{j^{\prime}}}\left[B_{j j^{\prime}}(\mathbf{z}, t) \rho\left(\mathbf{z}, t \mid \mathbf{y}, t^{\prime}\right)\right],
\end{aligned}
$$


y el correspondiente proceso solución de la misma es llamado, matemáticamente, proceso difusivo. El vector $\boldsymbol{A}(\mathbf{z}, t)$ es llamado vector drift mientras que la matriz $\boldsymbol{B}(\mathbf{z}, t)$ es llamada matriz de difusión.

En el caso particular en que tanto $\boldsymbol{A}$ como $\boldsymbol{B}$ son constantes, se obtiene la ecuación de difusión normal:

$$
\begin{aligned}
\frac{\partial \rho}{\partial t}\left(\mathbf{z}, t \mid \mathbf{y}, t^{\prime}\right)= & -\sum_{j=1}^{N_{d}} \frac{\partial}{\partial z_{j}} A_{j} \rho\left(\mathbf{z}, t \mid \mathbf{y}, t^{\prime}\right) \\
& +\sum_{j, j^{\prime}} \frac{1}{2} \frac{\partial^{2}}{\partial z_{j} \partial z_{j^{\prime}}} B_{j j^{\prime}} \rho\left(\mathbf{z}, t \mid \mathbf{y}, t^{\prime}\right)
\end{aligned}
$$

Para una condición inicial puntual $\rho\left(\mathbf{z}, t_{0} \mid \mathbf{y}, t_{0}\right)=\delta(\mathbf{z}-\mathbf{y})$, la solución de la misma se corresponde con la distribución del movimiento Browniano (MB), estudiado en Einstein (1956) y también llamado proceso de Wiener:

$$
\begin{aligned}
\rho\left(\mathbf{z}, t \mid \mathbf{y}, t_{0}\right)= & {\left[(2 \pi)^{N_{d}} \operatorname{det}[\boldsymbol{B}]\left(t-t_{0}\right)\right]^{-1 / 2} } \\
& \times \exp \left[-\frac{1}{2\left(t-t_{0}\right)}\left(\mathbf{z}-\mathbf{y}-\boldsymbol{A}\left(t-t_{0}\right)\right)^{T} \boldsymbol{B}^{-1}\left(\mathbf{z}-\mathbf{y}-\boldsymbol{A}\left(t-t_{0}\right)\right)\right],
\end{aligned}
$$

donde el supraíndice $T$ denota la operación de trasposición. Además de la propiedad Markoviana y de continuidad de las trayectorias muestra debe decirse que el movimiento Browniano es Gaussiano, en el sentido de que para todo tiempo fijo, la densidad de probabilidad condicional es una función Gaussiana $N$-dimensional. También tiene las propiedades de poseer incrementos estacionarios e independientes.

De la Ec. (1.58) se observa que $\boldsymbol{B}$ es una matriz real simétrica y por lo tanto diagonalizable. Por lo tanto, sin pérdida de generalidad, se puede suponer que el tensor de difusión es diagonal, y entonces, solución (1.62) se convierte en $N$ procesos difusivos independientes dados por $\rho_{j}\left(z_{j}, t \mid y_{j}, t_{0}\right)=\mathcal{N}\left(z_{j} ; y_{j}+A_{j}\left(t-t_{0}\right), B_{j j}\left(t-t_{0}\right)\right)$ (para $\left.j=1,2, \ldots, N_{d}\right)$, donde se ha utilizado la función normal o Gaussiana de valor medio $\mu$ y varianza $\sigma^{2}$ :

$$
\mathcal{N}\left(x ; \mu, \sigma^{2}\right)=\frac{1}{\sqrt{2 \pi \sigma^{2}}} e^{-\frac{(x-\mu)^{2}}{2 \sigma^{2}}} .
$$

Cada uno de estos procesos presenta un crecimiento lineal de la varianza (1.49):

$$
\sigma_{j}^{2}(t)=B_{j j}\left(t-t_{0}\right)
$$

y en el caso particular en que $\boldsymbol{A}=\mathbf{0}$, se cumple además que $\gamma_{j}^{2}(t)=\sigma_{j}^{2}(t)$.

Se dice que una variable estocástica es un proceso de difusión normal si su varianza crece linealmente con el tiempo ${ }^{2}$. Según el desarrollo previo, el proceso descripto por Ec. (1.62) presenta difusión normal.

A modo de explicar la diferencia en un factor $1 / 2$, entre la definición de coeficiente de difusión dada por Ec. (1.40) y la dada implícitamente por Ec. (1.64), se explicarán las dos convenciones existentes, considerando sólo el caso unidimensional.

\footnotetext{
${ }^{2}$ Otra definición usual considera difusión normal a aquella cuyo desplazamiento cuadrático medio es una función lineal del tiempo.
} 
Para $N_{d}=1$, la ecuación de F-P (1.60) se reduce a:

$$
\begin{aligned}
\frac{\partial \rho}{\partial t}\left(z, t \mid y, t^{\prime}\right)= & -\frac{\partial}{\partial z}\left[A(z, t) \rho\left(z, t \mid y, t^{\prime}\right)\right] \\
& +\frac{1}{2} \frac{\partial^{2}}{\partial z^{2}}\left[B(z, t) \rho\left(z, t \mid y, t^{\prime}\right)\right],
\end{aligned}
$$

En la misma, el coeficiente de difusión es $B(z, t)$, y en la situación de difusión normal la evolución de la varianza satisface:

$$
\sigma^{2}(t)=B\left(t-t_{0}\right)
$$

Esta es la convención que se utilizará en el capítulo 5.

La otra convención está dada por la siguiente ecuación de F-P:

$$
\begin{aligned}
\frac{\partial \rho}{\partial t}\left(z, t \mid y, t^{\prime}\right)= & -\frac{\partial}{\partial z}\left[A(z, t) \rho\left(z, t \mid y, t^{\prime}\right)\right] \\
& +\frac{\partial^{2}}{\partial z^{2}}\left[B(z, t) \rho\left(z, t \mid y, t^{\prime}\right)\right],
\end{aligned}
$$

En la misma, el coeficiente de difusión es $B(z, t)$, y en la situación de difusión normal la evolución de la varianza satisface:

$$
\sigma^{2}(t)=2 B\left(t-t_{0}\right)
$$

Es esta última convención la que fue utilizada en la sección 1.3.1 al definir los coeficientes de difusión y es la que será aplicada en los experimentos numéricos del capítulo 4.

El segundo proceso ideal obtenible de la ecuación diferencial de C-K consiste en pedir que tanto $W$ como $\boldsymbol{B}$ sean nulos, obteniéndose la ecuación de Liouville:

$$
\frac{\partial \rho}{\partial t}\left(\mathbf{z}, t \mid \mathbf{y}, t^{\prime}\right)=-\sum_{j=1}^{N_{d}} \frac{\partial}{\partial z_{j}}\left[A_{j}(\mathbf{z}, t) \rho\left(\mathbf{z}, t \mid \mathbf{y}, t^{\prime}\right)\right] .
$$

Esta ecuación tiene la particularidad de que, para una condición inicial puntual, su solución es $\rho\left(\mathbf{z}, t \mid \mathbf{y}, t^{\prime}\right)=\delta[\mathbf{z}-\mathbf{x}(t)]$, donde $\mathbf{x}(t)$ es la solución del PVI:

$$
\frac{d \mathbf{x}(t)}{d t}=\boldsymbol{A}(\mathbf{x}, t), \quad \mathbf{x}\left(t^{\prime}\right)=\mathbf{y}
$$

Por lo tanto, la ecuación de F-P, para tensor de difusión nulo y una condición inicial deltiforme, deviene en un sistema completamente determinista, modelable por un sistema de ecuaciones diferenciales ordinarias. Esto implica que, en el marco de la teoría de procesos estocásticos, un sistema de ecuaciones Hamiltonianas (1.2.1), no presenta ninguna característica difusiva.

Sin embargo, la posibilidad de que algún proceso Hamiltoniano pueda modelarse por una ecuación de difusión tipo F-P no debe descartarse. Si bien la ecuación de C-K asociada a un sistema Hamiltoniano no posee término difusivo, el introducir promediados en ciertas variables, o el adoptar un granulado grueso en el espacio de fases, puede permitir obtener una ecuación de F-P que modele, efectivamente, a la densidad de probabilidad de alguna de las quasi-acciones del sistema. Uno de los resultados de esta tesis (capítulo 5) está basado en este formalismo estocástico para obtener una Ec. de F-P para modelar el proceso difusivo de 
una acción perturbada de un mapa simpléctico 4D. En la sección 1.4.6 se presentan trabajos previos con este enfoque.

El tercer proceso deducible de la Ec. (1.59) corresponde a $\boldsymbol{A}$ y $\boldsymbol{B}$ nulos y $W$ distinto de cero, obteniéndose la llamada ecuación Maestra:

$$
\frac{\partial \rho}{\partial t}\left(\mathbf{z}, t \mid \mathbf{y}, t^{\prime}\right)=\int d \mathbf{x}\left[W(\mathbf{z} \mid \mathbf{x}, t) \rho\left(\mathbf{x}, t \mid \mathbf{y}, t^{\prime}\right)-W(\mathbf{x} \mid \mathbf{z}, t) \rho\left(\mathbf{z}, t \mid \mathbf{y}, t^{\prime}\right)\right]
$$

que describe un proceso de saltos y cuya solución puede consultarse en Gardiner (2004). Además, en el mismo se demuestra como obtener una de las versiones del clásico proceso estocástico random walk (RW), la versión de tiempo continuo, considerando un proceso de saltos unidimensional en el que el espacio de fases son los números enteros y donde se adopta como distribución de los saltos (por unidad de tiempo) a:

$$
W(m \mid n, t)= \begin{cases}\text { cte. } & \text { si } m=n \pm 1 \\ 0 & \text { si } m \neq n \pm 1\end{cases}
$$

Además, considerando un tipo de límite en el cual se hace tender el tamaño de los pasos a cero, es posible demostrar que el RW de tiempo continuo se transforma en un proceso de Wiener. Esto es un ejemplo de que existen procesos de saltos que están relacionados con procesos difusivos, así como también los hay relacionados con procesos deterministas. En el capítulo 5 se presentarán otros tipos de procesos estocásticos.

Una vez que se conoce la función $\rho\left(\mathbf{z}, t \mid \mathbf{y}, t_{0}\right)$, es posible computar el valor de expectación (condicional), $E[\cdot]$, de cualquier función, $f$, del vector estocástico mediante la expresión:

$$
E[f(\boldsymbol{X}(t))]=\int f(\mathbf{x}) \rho\left(\mathbf{x}, t \mid \mathbf{y}, t_{0}\right) d \mathbf{x} .
$$

Este tipo de promedio teórico será utilizado solamente en el capítulo 5. La principal diferencia entre el promedio de ensambles $(\langle\cdot\rangle)$ y el valor de expectación, radica en que el primero consiste en un promedio numérico sobre un número finito de trayectorias muestra (realizaciones del proceso estocástico), mientras que el segundo consiste en un promedio teórico, realizado sobre todas las posibles trayectorias muestra. En el límite de un número de trayectorias muestra, o equivalentemente de partículas de prueba, yendo a infinito, el promedio de ensambles debe converger hacia el valor de expectación.

El nombre de proceso difusivo no está limitado, ni a aquel que satisface la ecuación de F-P, ni a aquel que satisface la ecuación de C-K. Por el contrario, existe un amplio espectro de procesos difusivos anómalos y algunos de ellos han sido utilizados como herramientas para modelar la difusión en sistemas Hamiltonianos y, en particular, en sistemas Hamiltonianos Astrofísicos. A continuación se citarán, sin definir, sólo unos pocos de dichos procesos.

Chechkin et al. (2008) presenta una introducción a los viajes de Lévy (Lévy flights). Metzler et al. (2007) analiza a estos últimos, junto con las caminatas de Lévy (Lévy walks), los procesos subdifusivos y las ecuaciones de F-P fraccionales.

Mandelbrot y van Ness (1968) introduce por primera vez el movimiento Browniano fraccional (MBF), a partir de una integral (estócástica) particular del MB. Los MBFs poseen una desviación estándar cuya dependencia temporal esta dada por:

$$
\sigma_{v}(t) \propto t^{v}
$$


donde $v$ es llamado exponente de Hurst. Estos procesos pueden clasificarse en tres familias diferentes de acuerdo al valor de este exponente. Si $v=1 / 2$ entonces el MBF se reduce al $\mathrm{MB}$, por lo que la difusión es normal. Si $0<v<1 / 2$, se dice que el proceso es subdifusivo, mientras que si $1 / 2<v<1$ el proceso se clasifica como superdifusivo.

Anteriormente, al analizar la reducción a la Ec. de Liouville de la Ec. de F-P, se discutió acerca de si un sistema dinámico puede o no presentar aspectos difusivos en el marco estocástico. En esta tesis, al hablar de difusión caótica (o determinista) en sistemas Hamiltonianos casi-integrables, el concepto de difusión se referirá simplemente a que una variable acción del Hamiltoniano no perturbado $H_{0}(\boldsymbol{I})$ es capaz de experimentar una variación macroscópica mayor a la de la amplitud de la perturbación en la región bajo estudio. Es decir, se adopta una definición más "débil" de difusión, ya que no se exige que la densidad de probabilidad de $\boldsymbol{I}$ obedezca ecuación macroscópica alguna. Tampoco se exige que las cantidades estadísticas, como ser la varianza o la desviación media cuadrática, posean una determinada dependencia temporal. En resumen, se ha adoptado la noción de que existe difusión si y sólo si existe transporte en el espacio de acciones no perturbadas. Esta definición es la utilizada por Morbidelli (2006), Chirikov (1979), Giordano y Cincotta (2004), Cincotta (2002), Cachucho et al. (2010), Zaslavsky (2002), Meiss (1992), entre otros.

\subsubsection{Permisos topológicos y dinámicos}

En esta sección se hablará sobre la posibilidad de tener difusión en un sistema Hamiltoniano casi-integrable. Se ilustrará cuán entrelazados entre sí están los conceptos dinámicos y los topológicos al momento de establecer una cota a la región del espacio de fases accesible a una dada trayectoria. La situación es que a medida que se van conociendo nuevas características dinámicas se van pudiendo definir nuevos objetos matemáticos, como son por ejemplo los toros KAM, los cantori, las órbitas periódicas, las superficies de energía constante, las variedades hiperbólicas y elípticas, las superficies resonantes, las capas estocásticas, la red de resonancias, etc.

A medida que se conoce la existencia de nuevos objetos matemáticos se intenta establecer su cantidad (medida Lebesgue) y su ubicación en el espacio de fases. Esto permite determinar la topología de los conjuntos de objetos de un mismo tipo para, posteriormente, analizar si la distribución topológica de cierto tipo de objeto impone restricciones dinámicas a las trayectorias que tienen lugar en su complemento.

A su vez, los conocimientos topológicos permiten realizar nuevos estudios analíticos, como ser la realización de formas normales específicas, con la intención de encontrar nueva información dinámica.

En un sistema Hamiltoniano integrable $H_{0}(\boldsymbol{I})$ la condición de conservación de energía se expresa $H_{0}(\boldsymbol{I})=H_{0}\left(\boldsymbol{I}_{0}\right)=h$, donde $h$ es el valor de la energía para la condición inicial $\boldsymbol{I}_{0}$. Dicha ecuación determina una variedad de dimensión $n_{d}-1$ en el espacio de acciones, que notaremos $\mathcal{I}_{0}$. Cuando $n_{d}=3$ dicha variedad es usualmente llamada superficie de energía y cuando $n_{d}=2$ dicha variedad deviene en una curva. El espacio conformado por el producto directo entre $\mathcal{I}_{0}$ y $\mathbb{T}^{n_{d}}$ determina una variedad de dimensión $N_{d}-1=2 n_{d}-1$ contenida en el espacio de fases, que satisface que la energía es constante en cada punto de la misma (ya que el Hamiltoniano no depende de los ángulos).

$\mathrm{Al}$ encender la perturbación, haciendo $\epsilon$ no nulo, en el Hamiltoniano casi-integrable $H(\boldsymbol{I}, \boldsymbol{\theta})=H_{0}(\boldsymbol{I})+\epsilon H_{1}(\boldsymbol{I}, \boldsymbol{\theta})$ presentado en Ec. (1.13), sigue valiendo el hecho de que la variedad de energía constante tiene dimensión $N_{d}-1$. Sin embargo, debido a que ahora $H$ 
depende de los ángulos, la proyección de dicha variedad sobre el espacio de acciones, que notaremos $\mathcal{I}_{\epsilon}$, no posee dimensión $n_{d}-1$. Siguiendo con el ejemplo de $n_{d}=3$, para $\epsilon \neq 0$, se tiene que $\mathcal{I}_{\epsilon}$ posee "volumen". Incluso, sucede que un mismo valor de $\boldsymbol{I}$ corresponde a dos valores de la energía distintos, si está acompañado de ángulos distintos: $H\left(\boldsymbol{I}, \boldsymbol{\theta}_{1}\right)=h_{1}$ pero $H\left(\boldsymbol{I}, \boldsymbol{\theta}_{2}\right)=h_{2}$. La condición de conservación de energía puede expresarse como

$$
H_{0}(\boldsymbol{I})-H_{0}\left(\boldsymbol{I}_{0}\right)=-\epsilon\left[H_{1}(\boldsymbol{I}, \boldsymbol{\theta})-H_{1}\left(\boldsymbol{I}_{0}, \boldsymbol{\theta}_{0}\right)\right] .
$$

Por lo tanto, definiendo como $C$ a una cota superior de $\left|H_{1}(\boldsymbol{I}, \boldsymbol{\theta})-H_{1}\left(\boldsymbol{I}_{0}, \boldsymbol{\theta}_{0}\right)\right|$, se tiene que dicha condición de conservación impone una cota implícita en el comportamiento de $\boldsymbol{I}$ :

$$
\left|H_{0}(\boldsymbol{I})-H_{0}\left(\boldsymbol{I}_{0}\right)\right| \leq \epsilon C .
$$

La misma establece que sea cual sea la dinámica, la acción no podrá alejarse de la superficie de energía no perturbada $\left(\mathcal{I}_{0}\right)$ en más de una cantidad del orden de $\epsilon C$. Es decir, la consideración energética establece que $\mathcal{I}_{\epsilon}$ es una "capa" alrededor de $\mathcal{I}_{0}\left(\mathcal{I}_{\epsilon} \supset \mathcal{I}_{0}\right)$. Sin embargo, utilizando sólo estas consideraciones energéticas no puede predecirse qué puntos de $\mathcal{I}_{\epsilon}$ podría efectivamente alcanzar la trayectoria.

Las consideraciones energéticas anteriormente analizadas son válidas para cualquier valor de la perturbación. En cambio, las consideraciones que se darán a continuación dependerán de si las resonancias perturbadas se hallan o no en régimen de overlap (capítulo 3). A medida que se va aumentando el valor de $\epsilon$, las resonancias y las capas estocásticas se van ensanchando y solapando mutuamente. El hecho de que dos o más resonancias entren en régimen de overlap no sólo depende de $\epsilon$ sino también del tamaño de los coeficientes asociados a esos armónicos resonantes en la perturbación $\left(H_{1}\right)$, de la distancia de las resonancias entre sí y de la multiplicidad de las mismas, por nombrar algunos factores. Sin embargo, es posible determinar un valor crítico de $\epsilon$, que se llamará $\epsilon_{c}$, de forma tal que si $\epsilon>\epsilon_{c}$ entonces el sistema se halla mayoritariamente en régimen de overlap. Este régimen también es llamado régimen de Chirikov, mientras que la situación en que $\epsilon<\epsilon_{c}$ es llamada régimen de Nekhoroshev. La determinación de tal valor se ha realizado mediante el análisis del indicador dinámico FLI en Froeschlé et al. (2000), para un sistema Hamiltoniano 3DoF. En Guzzo et al. (2002) se aplicó este método junto con uno basado en una formulación espectral del teorema de Nekhoroshev (sección 1.4.3) para un flujo Hamiltoniano, obteniéndose dos valores de $\epsilon_{c}$ que difieren entre sí en un orden de magnitud. En Mestre et al. (2009) se determina el valor $\epsilon_{c}$ para un flujo Hamiltoniano 3DoF, utilizando el criterio de overlap de Chirikov obteniéndose acuerdo total con el obtenido previamente en Giordano y Cincotta (2004), donde se utilizó el indicador dinámico MEGNO.

A continuación se analizará el régimen de Nekhoroshev. En la sección 1.2.6 se definieron los toros KAM y se habló del teorema asociado. Recordemos que un toro KAM, para $\epsilon$ suficientemente pequeño, es estable por tiempo indefinido, y por lo tanto, surge la pregunta de si esta estabilidad podría imponer barreras a órbitas con condiciones iniciales que no pertenecen a toros KAM. El hecho de que la dimensión de un toro KAM es $n_{d}$ mientras que la conservación de $H$ conlleva una restricción a una variedad de dimensión $2 n_{d}-1$, implica que el complemento del toro KAM posee dimensión $n_{d}-1$. Por lo tanto, este complemento queda dividido en partes disconexas solamente en el caso $n_{d}=2$.

Este es un ejemplo de como el conocimiento dinámico producto de aplicar la forma normal de Kolmogorov (que se utiliza para demostrar teorema KAM) permitió definir objetos matemáticos (toros KAM), y junto con un posterior análisis topológico, fue posible imponer 
fuertes restricciones en la dinámica de sistemas con dos grados de libertad. Sin embargo, para $n_{d}>2$, no necesariamente existe la posibilidad de que haya trayectorias que se difundan libremente en el complemento de los toros KAM. De hecho, en el entorno de cada toro invariante es posible construir una forma normal de Birkhoff, de forma tal que el posterior análisis dinámico del resto al orden óptimo, impone una fuerte restricción a la velocidad de difusión. Morbidelli y Giorgilli (1995) encuentran que el tamaño de dicha velocidad es superexponencialmente pequeño respecto a la inversa de la distancia al toro invariante; es decir, proporcional a $\exp (\exp (-1 / r))$, donde $r$ es la distancia al toro invariante. Entonces, para $\epsilon$ suficientemente pequeño, la alta densidad de toros KAM en ciertas regiones del espacio de fases actúa como barrera a la difusión.

En 1.4.4 se mostrará como la construcción de una forma normal a primer orden en $\epsilon$, en entorno de acción resonante simple, consiste en el Hamiltoniano de un péndulo simple (sección 1.2.7). Dicha normalización permite definir otro tipo de objetos matemáticos, llamados toros resonantes invariantes, que al igual que los toros KAM, son densos en el espacio de fases. Previamente, en 1.2.3, se definió la red de resonancias. La misma existe para perturbación nula. Para $\epsilon \neq 0$, según se explicó en 1.2.8, aparecen capas estocásticas asociadas a las separatrices de las resonancias no pertubadas que envuelven a las superficies resonantes perturbadas. En esta tesis, se llamará red de Arnold (Arnold web) al conjunto formado por la intersección de todas las superficies resonantes perturbadas con la variedad de energía perturbada constante $(H(\boldsymbol{I}, \boldsymbol{\theta})=h)$. Esta red incluye a todas las capas estocásticas que serán creadas (con distintos grados de intensidad) en los entornos de las infinitas resonancias del sistema no perturbado. Chirikov (1979) llama a esta red resonance network o resonance web, pero nosotros reservamos este último término para las superficies resonantes sin perturbar. Por lo dicho anteriormente, para $n_{d}=2$ las órbitas caóticas pertenecientes a dicha red estarán localmente acotadas por los toros KAM que aún persisten, mientras que para casos de dimensionalidad superior las órbitas pertenecientes a la capa estocástica no tienen ninguna barrera dinámica que les impida recorrer la red de Arnold. Sin embargo, si existen restricciones teóricas en la velocidad con la que pueden difundirse o transportarse (Nekhoroshev 1977, Benettin and Gallavotti 1986).

Una definición equivalente de red de Arnold, dada por Guzzo et al. (2005), es la siguiente. Dados valores de $\epsilon$ suficientemente pequeños de forma tal que sean válidos ambos los teoremas KAM y de Nekhoroshev (sección 1.4.3), se tiene que los toros invariantes llenan casi todo el espacio de fases, garantizando órbitas quasi-periódicas (y acotadas) para la mayoría de las condiciones iniciales. La red de Arnold es el complemento de este conjunto respecto a $H(\boldsymbol{I}, \boldsymbol{\theta})=h$. La misma incluye tanto órbitas regulares como caóticas. Entre las primeras se pueden nombrar aquellas órbitas que tienen lugar en los regímenes oscilatorios de las resonancias perturbadas y las que tienen lugar en los cantori. Entre las segundas se encuentran las órbitas caóticas que suceden en las capas estocásticas asociadas a resonancias perturbadas.

Debido a la dificultad de visualizar la red de Arnold para $n_{d} \geq 2$, es una práctica común aprovechar el hecho de que para $\epsilon$ pequeños la variedad $\mathcal{I}_{\epsilon}$ no difiere mucho de su contraparte no perturbada, $\mathcal{I}_{0}$. Lo que se hace es trabajar sólo en el espacio de acciones graficando la red de Arnold como la itersección de la superficies resonantes perturbadas con $\mathcal{I}_{0}$.

Ahora se analizará qué sucede cuando el sistema se halla en régimen de Chirikov. Chirikov (1979) estudió en el mapa estándar, la difusión global que tiene lugar cuando la perturbación es suficientemente grande como para romper todos los toros invariantes que podrían separar entre sí las regiónes de movimiento caótico que suceden en las capas estocásticas. En este caso, para cualquier valor de $n_{d}$, la componente caótica queda mayoritariamente conectada. 
Resumiendo lo analizado en esta sección, se tiene que en régimen de overlap global de resonancias, existe difusión macroscópica y global, independientemente de la dimensionalidad del Hamiltoniano. Por otro lado, en régimen de ausencia de overlap global, para $n_{d}=2$ la difusión, en caso de existir, está limitada localmente a un número finito de resonancias, mientras que para $n_{d}>2$, sólo tiene permiso de suceder en la red de Arnold.

\subsection{Teorías de la difusión}

A continuación se mencionarán algunas de las teorías de la difusión en el espacio de acciones de sistemas Hamiltonianos junto con parte de los trabajos experimentales que se han realizado al respecto. Entre las teorías que no se detallarán se encuentran la difusión adiabática, la difusión modulada y métodos que utilizan funciones características.

\subsubsection{Mecanismo de Arnold}

Para un sistema Hamiltoniano 2.5DoF (en este caso $n_{d} \notin \mathbb{N}$ pues el sistema no es autónomo), Arnold (1964) demuestra la existencia de un mecanismo que genera una inestabilidad macroscópica para ciertas condiciones iniciales particulares.

El sistema Hamiltoniano es el siguiente:

$$
\begin{gathered}
H\left(I_{1}, I_{2}, \theta_{1}, \theta_{2}, t\right)=\frac{1}{2}\left(I_{1}^{2}+I_{2}^{2}\right)+\epsilon\left(\cos \theta_{1}-1\right)\left(1+\mu B\left(\theta_{2}, t\right)\right) \\
B\left(\theta_{2}, t\right)=\sin \theta_{2}+\cos t .
\end{gathered}
$$

Para $\epsilon=\mu=0$ el sistema consiste en dos rotadores libres, donde $I_{1}$ e $I_{2}$ son integrales de movimiento. Para $\mu=0$ y $\epsilon \neq 0$ este sistema consiste en un rotador libre y un péndulo libre, con integrales de movimiento, respectivamente, $I_{2}$ y $J$, donde

$$
J \equiv \frac{1}{2} I_{1}^{2}+\epsilon\left(\cos \theta_{1}-1\right)=\text { const. }
$$

La separatriz de este péndulo, o resonancia con frecuencia $\omega_{1}=0$, corresponde a resolver $J=0$ en (1.79). Siguiendo el razonamiento de Chierchia (1995), se tiene que el toro resonante, etiquetable por $\omega \equiv I_{2}$ :

$$
\mathcal{T}_{\omega} \equiv\left\{I_{1}=0=\theta_{1}, I_{2}=\omega, \theta_{2} \in \mathbb{T}\right\}
$$

es invariante por $H$ para todo valor de $\epsilon$ y $\mu$, y por lo tanto, en cada toro la dinámica esta dada por:

$$
\left(I_{1}, I_{2}, \theta_{1}, \theta_{2}\right)=\left(0, \omega, 0, \theta_{2}(0)+\omega t\right) .
$$

Los toros $\mathcal{T}_{\omega}$ son llamados toros abigotados (whiskered tori), ya que son las variedades asintóticas de las separatrices correspondientes a los péndulos simples, con Hamiltoniano $J$ (1.79). Para $\mu=0$, tanto las separatrices como los toros abigotados correspondientes a diferentes valores de $\omega$ se encuentran aisladas unas de las otras.

Dado un conjunto de toros abigotados, una cadena de transición es, básicamente, la existencia de órbitas que pueden pasar por entornos arbitrariamente pequeños de todos estos toros en un tiempo finito.

El mecanismo de Arnold consiste en que para todo $\epsilon, r>0$ existe un $\mu_{0}>0$ tal que para todo $0<\mu<\mu_{0}$ existen toros invariantes $\mathcal{T}_{\omega}$ y $\mathcal{T}_{\omega^{\prime}}$, con $\left|\omega-\omega^{\prime}\right|>r$, que están conectados por una cadena de transición. 
Giorgilli (1990) analiza el mecanismo de Arnold para la difusión. Además, presenta el contexto general del problema de la estabilidad en sistema casi-integrables.

\subsubsection{Difusión de Arnold}

En Nekhoroshev (1977), Chirikov (1979), Guzzo et al. (2005) y Efthymiopoulos (2008) se entiende la difusión de Arnold como aquella difusión que tiene lugar sobre la red de Arnold de un sistema que satisface simultáneamente las hipótesis de ambos los teoremas KAM y de Nekhoroshev (secciones 1.2.6 y 1.4.3, respectivamente). Según 1.3.3, es la difusión que tiene lugar en sistemas Hamiltonianos casi-integrables que se hallan en régimen de Nekhoroshev $\left(\epsilon<\epsilon_{c}\right)$.

Esta no es la única definición de difusión de Arnold presente en la literatura. Chierchia and Gallavotti (1994) definen un sistema a priori inestable como un sistema cuya parte no perturbada posee separatrices o variedades hiperbólicas en general. Esto implica la presencia de órbitas periódicas inestables y sus respectivas variedades estables e inestables. En este tipo de sistemas logran probar la existencia de una inestabilidad (macroscópica) en las acciones tal que son transportadas en una cantidad $\mathcal{O}(1)$ en un tiempo finito. A su vez logran probar que la dependencia de la velocidad de difusión tiende en forma continua a 0 con $\epsilon$. En Guzzo et al. (2009) se estudia la difusión en este tipo de sistemas.

Según lo visto en sección 1.4.1, para un valor fijo finito y no nulo de $\epsilon$, utilizando sólamente a $\mu$ como parámetro perturbativo, se tiene que el ejemplo de Arnold pertenece a la clase de sistemas a priori inestables. Es sobre este sistema no perturbado que Arnold prueba la existencia de una inestabilidad de orden unidad. Por lo tanto, de acuerdo con Lochak (1999), el ejemplo de Arnold para la difusión de Arnold, consiste en un sistema que posee hiperbolicidad (integrable) aún antes de ser perturbado. Sin embargo, el modelo de Arnold no es representativo de todos los sistemas a priori inestables (o a priori hiperbólicos). Guzzo et al. (2009) menciona que la variedad invariante hiperbólica a lo largo de la cual Arnold prueba la existencia de difusión está llena de toros invariantes para todo valor del parámetro perturbativo $(\mu)$ y que la restricción del sistema dinámico a la variedad invariante es integrable. Esta situación no corresponde con el caso general.

\subsubsection{Teoría de Nekhoroshev}

Nekhoroshev (1977) establece una cota a la velocidad de difusión de las acciones para un sistema Hamiltoniano con ciertas propiedades que a continuación se detallarán. Dicha cota es global a todo el espacio de fases. La rigurosa enunciación del teorema requiere el estudio previo de una cantidad de conceptos matemáticos y estudios geométricos de la estructura resonante, que exceden las incumbencias de esta tesis. Por tal motivo, se enunciará una versión más relajada del teorema, tal como es enunciado en Morbidelli (2006).

Sea un sistema Hamiltoniano casi-integrable $n_{d}$-dimensional de la forma (1.13) $H(\boldsymbol{I}, \boldsymbol{\theta})=$ $H_{0}(\boldsymbol{I})+\epsilon H_{1}(\boldsymbol{I}, \boldsymbol{\theta})$, donde $\boldsymbol{I}, \boldsymbol{\theta}$ están definidas en una región $\mathcal{R} \equiv \mathcal{G} \wedge \mathbb{T}^{n_{d}}$ un dominio de analiticidad de $H$, donde $\mathcal{G}$ (espacio de acciones) es un dominio abierto y acotado de $\mathbb{R}^{n_{d}}$. Sea $\mathcal{G}-\Delta$ el conjunto de puntos $\boldsymbol{I}$ tales que un entorno de radio $\Delta$ centrado en ellos esté completamente contenido en $\mathcal{G}$. Sea $C(\boldsymbol{I})$ la matriz Hessiana de $H_{0}(\boldsymbol{I})$ y asumamos la hipótesis de convexidad:

$$
C(\boldsymbol{I}) \mathbf{v} \cdot \mathbf{v} \neq \mathbf{0} \quad \forall \boldsymbol{I} \in \mathcal{G} \quad \text { y } \quad \forall \mathbf{v} \in \mathbb{R}^{\mathrm{n}_{\mathrm{d}}} \backslash \mathbf{0} .
$$


Luego, existen constantes positivas $\epsilon_{*}, \alpha, \beta, a$ y $b$ tales que para todo $\epsilon<\epsilon_{*}$ vale que

$$
|\boldsymbol{I}(t)-\boldsymbol{I}(0)| \leq \Delta \equiv \alpha \epsilon^{a},
$$

para todo $\boldsymbol{I}(0) \in \mathcal{G}-\Delta$ y para todo $|t| \leq T(\epsilon)$ donde

$$
T(\epsilon)=\beta\left(\frac{\epsilon_{*}}{\epsilon}\right)^{1 / 2} \exp \left(\frac{\epsilon_{*}}{\epsilon}\right)^{b} .
$$

El tiempo de estabilidad $T$ crece exponencialmente respecto a $\epsilon_{*} / \epsilon$. Por esta razón, tan pronto como $\epsilon$ es un poco menor que el umbral $\epsilon_{*}$, el tiempo de estabilidad se vuelve extremadamente largo y puede llegar a exceder el tiempo de vida del sistema físico.

Froeschlé et al. (2005), Lega et al. (2003) y Guzzo et al. (2005) estudiaron numéricamente la difusión de Arnold en sistemas que satisfacen las hipótesis de ambos el teorema KAM y el de Nekhoroshev. En particular, en Lega et al. (2003), detectan numéricamente, que el coeficiente de difusión va a cero más rápido que cualquier ley de potencias $\left(\mathcal{D}(\epsilon) \propto \epsilon^{b}\right)$ y por lo tanto es compatible con la ley exponencial $\left(\mathcal{D}(\epsilon) \propto \exp \left(-1 / \epsilon^{a}\right)\right.$ para alguna constante a). Efthymiopoulos (2008) construye una forma normal resonante hasta el orden óptimo de normalización para la misma resonancia estudiada por Lega et al. (2003). Computa el tamaño del resto al orden óptimo, $\left|R_{\text {opt }}\right|$ en función de $\epsilon$, encontrando que $\left|R_{o p t}\right| \propto \exp \left(-1 / \epsilon^{0.21}\right)$ para $\epsilon$ en un subintervalo perteneciente al régimen de Nekhoroshev. Posteriormente, ajustando sus datos con los de Lega et al. (2003), encuentra que la dependencia del coeficiente de difusión con el resto esta dada por $\mathcal{D} \propto\left|R_{\text {opt }}\right|^{3}$.

En resumen, la teoría de Nekhoroshev no es, rigurosamente, una teoría de la difusión. $\mathrm{Su}$ resultado consiste en asegurar que, en caso de existir difusión, esta no puede tener una velocidad mayor que cierta cota. Una de las desventajas de la teoría es que la cota es global, no teniendo en cuenta las particularidades de la región que se pretende estudiar.

Giorgilli (1990) presenta una demostración simple del Teorema de Nekhoroshev para un sistema Hamiltoniano tal que $H_{0}$ consiste en $n_{d}$ osciladores armónicos desacoplados, por lo que es isócrono.

\subsubsection{Teoría de Chirikov para la difusión de Arnold}

Chirikov (1979) propone un método semiempírico para predecir el coeficiente de difusión de Arnold presente en un sistema Hamiltoniano multidimensional genérico, de la forma dada por las Ecs. (1.13) y (1.20). Esta teoría ha sido revisada en Cincotta (2002) en un contexto astronómico.

Su teoría está basada en cuatro conceptos fundamentales. Uno de ellos es la determinación de la geometría resonante en el entorno de las condiciones iniciales. El segundo es un desarrollo de series de potencias hasta segundo orden en las acciones y hasta primer orden en el parámetro perturbativo, basado en la hipótesis de que ambas cantidades poseen magnitudes comparables. El tercero es el modelado de la dinámica que sucede en una capa estocástica mediante el uso de un mapa simpléctico específicamente construido. El último es una aproximación sobre el grado de aleatoriedad de la fase correspondiente a este mapa. A continuación se brinda una versión para tres grados de libertad de los primeros dos conceptos de la teoría, que serán de utilidad para el resto de la tesis.

Sea $\boldsymbol{m}_{g}$ un armónico presente en $H_{1}$ que genera una resonancia aislada (sección 1.2.5). Entonces, se reescribe al sistema así: 


$$
\begin{aligned}
& H(\boldsymbol{I}, \boldsymbol{\theta})=H_{0}(\boldsymbol{I})+\epsilon V_{\boldsymbol{m}_{g}}(\boldsymbol{I}) \cos \left(\boldsymbol{m}_{g} \cdot \theta\right)+\epsilon V(\boldsymbol{I}, \boldsymbol{\theta}) \\
& V(\boldsymbol{I}, \boldsymbol{\theta})=\sum_{\boldsymbol{m} \neq \boldsymbol{m}_{g}} V_{\boldsymbol{m}}(\boldsymbol{I}) \cos (\boldsymbol{m} \cdot \theta) .
\end{aligned}
$$

A continuación se realiza una transformación canónica lineal, para cambiar de las variables ángulo-acción de $H_{0}$ hacia variables $(\boldsymbol{p}, \boldsymbol{\phi})$. Sea $\boldsymbol{I}^{r}$ una acción resonante en cuyo entorno se pretende estudiar la difusión, sea $\boldsymbol{\omega}^{r} \equiv \boldsymbol{\omega}\left(\boldsymbol{I}^{r}\right)$ la frecuencia correspondiente y sea $\boldsymbol{n}_{g}$ el vector normal a su superficie resonante, llamada $\mathcal{S}_{g}$. Este último puede computarse de la forma:

$$
\boldsymbol{n}_{g}=\left[\frac{\partial}{\partial \boldsymbol{I}}(\boldsymbol{m} \cdot \boldsymbol{\omega}(\boldsymbol{I}))\right]_{\mid \boldsymbol{I}=\boldsymbol{I}^{r}}
$$

Además, se definen los vectores:

$$
\begin{aligned}
& \boldsymbol{\mu}_{1}=\boldsymbol{m}_{g}, \\
& \boldsymbol{\mu}_{2}=\boldsymbol{\omega}^{r} /\left|\boldsymbol{\omega}^{r}\right| \\
& \boldsymbol{\mu}_{3}=\left(\boldsymbol{n}_{g} \wedge \boldsymbol{\omega}^{r}\right) /\left|\boldsymbol{n}_{g} \wedge \boldsymbol{\omega}^{r}\right|,
\end{aligned}
$$

y la matriz $\Upsilon$ de $3 \times 3$, cuya fila $i$-ésima es el vector $\boldsymbol{\mu}_{i}$. Estos vectores son linealmente independientes sii $\boldsymbol{m}_{g}$ no es perpendicular a $\boldsymbol{n}_{g}$. Una manera de garantizar dicha condición geométrica, consiste en asumir que el Hamiltoniano $H_{0}(\boldsymbol{I})$ satisface la hipótesis de convexi$\operatorname{dad}(1.82)^{3}$.

Sea la función generatriz de la siguiente forma:

$$
F(\boldsymbol{p}, \boldsymbol{\theta}) \equiv \sum_{j=1}^{3}\left(I_{j}^{r}+\sum_{k=1}^{3} p_{k} \Upsilon_{k j}\right) \theta_{j}
$$

Luego, se define la transformación canónica

$$
\begin{aligned}
& \psi_{i}=\frac{\partial F}{\partial p_{i}}=\sum_{k} \Upsilon_{i k} \theta_{k}, \\
& I_{i}=\frac{\partial F}{\partial \theta_{i}}=I_{i}^{r}+\sum_{k} p_{k} \Upsilon_{k i},
\end{aligned}
$$

que escrita explícitamente adopta la forma:

$$
\begin{aligned}
& \psi_{i}=\frac{\partial F}{\partial p_{i}}=\sum_{k} \Upsilon_{i k} \theta_{k}, \\
& p_{i}=\sum_{k} \Upsilon_{i k}^{-T}\left(I_{k}-I_{k}^{r}\right),
\end{aligned}
$$

donde $\Upsilon^{-T} \equiv\left(\Upsilon^{T}\right)^{-1}$ denota la matriz inversa de la traspuesta de $\Upsilon$. Dicha transformación consiste en una traslación del origen hacia la acción resonante seguido de un cambio de base en el espacio de fases. La nueva base, que se llamará base de Chirikov esta formada por los vectores $\boldsymbol{\mu}_{i}$ y fue elegida por poseer una relación geométrica con la difusión que se pretende medir. $\boldsymbol{\mu}_{1}$ es igual al vector armónico resonante y por lo tanto, su ángulo conjugado, $\psi_{1} \equiv \boldsymbol{m}_{g} \cdot \boldsymbol{\theta}$, es la fase resonante del péndulo simple (sección 1.2.7) que modela a la resonancia. $\boldsymbol{\mu}_{2}$ es un versor perpendicular a la superficie de energía no perturbada $\left(\mathcal{I}_{0}\right) \cdot \boldsymbol{\mu}_{3}$ es un versor con la propiedad

\footnotetext{
${ }^{3}$ Como es el caso del Hamiltoniano analizado en el capítulo 4.
} 
de ser ortogonal a ambos $n_{g}$ y $\boldsymbol{\mu}_{2}$. En otras palabras, el tercer vector base es tangente a la curva intersección entre las superficies $\mathcal{S}_{g}$ y $\mathcal{I}_{0}$, en el punto $\boldsymbol{I}^{r}$.

La transformación (1.90) se puede reescribir matricialmente así:

$$
\begin{aligned}
& \boldsymbol{\psi}=\Upsilon \boldsymbol{\theta}, \\
& \boldsymbol{p}=\Upsilon^{-T}\left(\boldsymbol{I}-\boldsymbol{I}^{r}\right)
\end{aligned}
$$

Es posible notar la analogía entre esta transformación y la dada en Ec. (1.7). No necesariamente las variables $p_{i}$ y $\psi_{i}$ serán respectivamente acciones y ángulos del Hamiltoniano sin perturbar. Esto sólo sucede en el caso en que $\Upsilon^{-T}$ posee elementos enteros. De todas formas, siempre se cumple que las componentes de $\boldsymbol{p}$, al depender sólo de $\boldsymbol{I}$, son integrales de movimiento del Hamiltoniano sin peturbar.

Como se dijo recientemente, esta transformación es básicamente un cambio de base compuesto con una traslación. Esto puede demostrarse observando que las columnas de la matriz $\Upsilon^{T}$ se corresponden con los vectores de la base de Chirikov y, por lo tanto, $\Upsilon^{T}$ es la matriz de cambio de base desde esta base nueva hacia la canónica. Por este motivo, su inversa $\left(\Upsilon^{-T}\right)$ es la matriz de cambio de base en el sentido inverso. Entonces, se tiene que $p_{k}$ son las componentes del vector $\boldsymbol{I}-\boldsymbol{I}^{r}$ en la base de Chirikov.

En resumen, se tiene que las componentes del nuevo vector de momentos $(\boldsymbol{p})$ miden los desplazamientos que a continuación se detallan. $p_{1}$ mide el desplazamiento de $\boldsymbol{I}$ respecto de $\boldsymbol{I}^{r}$, en dirección del vector resonante, que es la misma que la dirección perpendicular a la capa estocástica. $p_{2}$ da el apartamiento en la dirección normal a $\mathcal{I}_{0} \cdot p_{3}$ da el desplazamiento en la dirección en que ocurre la difusión de Arnold.

Dado que la tranformación canónica no depende del tiempo, el nuevo Hamiltoniano es $\tilde{H}(\boldsymbol{p}, \boldsymbol{\psi}) \equiv H(\boldsymbol{I}(\boldsymbol{p}), \boldsymbol{\theta}(\boldsymbol{\psi}))$. Definiendo, para cada armónico, el vector $\boldsymbol{\nu}(\boldsymbol{m}) \equiv \Upsilon^{-T} \boldsymbol{m}$ se tiene que las fases asociadas son:

$$
\phi_{\boldsymbol{m}} \equiv \boldsymbol{m} \cdot \theta=\boldsymbol{\nu}(\boldsymbol{m}) \cdot \boldsymbol{\psi}
$$

y el nuevo Hamiltoniano puede reescribirse así:

$$
\begin{aligned}
& \tilde{H}(\boldsymbol{p}, \boldsymbol{\psi})=\tilde{H}_{0}(\boldsymbol{p})+\epsilon \tilde{V}_{\boldsymbol{m}_{g}}(\boldsymbol{p}) \cos \left(\boldsymbol{\psi}_{1}\right)+\epsilon \tilde{V}(\boldsymbol{p}, \boldsymbol{\psi}) \\
& \tilde{V}(\boldsymbol{p}, \boldsymbol{\psi})=\sum_{\boldsymbol{m} \neq \boldsymbol{m}_{g}} \tilde{V}_{\boldsymbol{m}}(\boldsymbol{p}) \cos (\boldsymbol{\nu}(\boldsymbol{m}) \cdot \boldsymbol{\psi}) .
\end{aligned}
$$

Utilizando algunas de las propiedades de ortogonalidad de la base de Chirikov, se prueba que $\boldsymbol{\omega}^{r} \cdot \boldsymbol{p}=p_{2}\left|\boldsymbol{\omega}^{r}\right|$ por lo que el desarrollo de Taylor alrededor de $\boldsymbol{p}=\mathbf{0}$ (o $\boldsymbol{I}=\boldsymbol{I}^{r}$ ) del Hamiltoniano no perturbado es

$$
\begin{aligned}
\tilde{H}_{0}(\boldsymbol{p})= & H_{0}\left(\boldsymbol{I}^{r}\right)+p_{2}\left|\boldsymbol{\omega}^{r}\right| \\
& +\frac{1}{2}\left(\frac{p_{1}^{2}}{M_{11}}+\frac{p_{2}^{2}}{M_{22}}+\frac{p_{3}^{2}}{M_{33}}\right)+\frac{p_{1} p_{2}}{M_{12}}+\frac{p_{1} p_{3}}{M_{13}}+\frac{p_{2} p_{3}}{M_{23}}+\mathcal{O}\left(|\boldsymbol{p}|^{3}\right),
\end{aligned}
$$

donde los denominadores están dados por

$$
\frac{1}{M_{k l}}=\sum_{i} \sum_{j} \Upsilon_{k i} \Upsilon_{l j} \frac{\partial \omega_{i}^{r}}{\partial I_{j}^{r}}=\frac{1}{M_{l k}}
$$


A continuación se desarrollan tres de ellas en términos de productos escalares entre vectores conocidos:

$$
\begin{aligned}
& \frac{1}{M_{11}}=\boldsymbol{m}_{g} \cdot \boldsymbol{n}_{g} \neq 0 \\
& \frac{1}{M_{12}}=\frac{1}{\left|\boldsymbol{\omega}^{r}\right|}\left(\boldsymbol{\omega}^{r} \cdot \boldsymbol{n}_{g}\right) \\
& \frac{1}{M_{13}}=\boldsymbol{\mu}_{\mathbf{3}} \cdot \boldsymbol{n}_{g}=0 .
\end{aligned}
$$

Una de las hipótesis simplificadoras consiste en suponer que $\mathcal{O}(|\boldsymbol{p}|)=\mathcal{O}(\sqrt{\epsilon})$, con lo cual el nuevo Hamiltoniano queda así:

$$
\begin{aligned}
\tilde{H}(\boldsymbol{p}, \boldsymbol{\psi})= & H_{0}\left(\boldsymbol{I}^{r}\right)+H_{\mathcal{C}}(\boldsymbol{p}, \boldsymbol{\psi})+\mathcal{O}\left(\epsilon^{3 / 2}\right), \\
H_{\mathcal{C}}(\boldsymbol{p}, \boldsymbol{\psi})= & p_{2}\left|\boldsymbol{\omega}^{r}\right| \\
& +\frac{1}{2}\left(\frac{p_{1}^{2}}{M_{11}}+\frac{p_{2}^{2}}{M_{22}}+\frac{p_{3}^{2}}{M_{33}}\right)+\frac{p_{1} p_{2}}{M_{12}}+\frac{p_{2} p_{3}}{M_{23}} \\
& +\epsilon \tilde{V}_{\boldsymbol{m}_{g}}(\mathbf{0}) \cos \left(\psi_{1}\right)+\epsilon \sum_{\boldsymbol{m} \neq \boldsymbol{m}_{g}} \tilde{V}_{\boldsymbol{m}}(\mathbf{0}) \cos (\boldsymbol{\nu}(\boldsymbol{m}) \cdot \boldsymbol{\psi}),
\end{aligned}
$$

donde $H_{\mathcal{C}}$ es el Hamiltoniano de Chirikov, que constituye el sistema específico de estudio de esta teoría. Este Hamiltoniano puede separarse en un Hamiltoniano del péndulo $H_{P}$, similar salvo en un signo al dado en 1.2.7, y una perturbación al mismo $V_{P}$, dados por:

$$
\begin{aligned}
H_{P}\left(p_{1}, \psi_{1}\right)= & \frac{1}{2} \frac{p_{1}^{2}}{M_{11}}+\epsilon \tilde{V}_{\boldsymbol{m}_{g}}(\mathbf{0}) \cos \left(\psi_{1}\right) \\
V_{P}(\boldsymbol{p}, \boldsymbol{\psi})= & p_{2}\left|\boldsymbol{\omega}^{r}\right|+\frac{p_{2}^{2}}{2 M_{22}}+\frac{p_{3}^{2}}{2 M_{33}}+\frac{p_{1} p_{2}}{M_{12}}+\frac{p_{2} p_{3}}{M_{23}} \\
& +\epsilon \sum_{\boldsymbol{m} \neq \boldsymbol{m}_{g}} \tilde{V}_{\boldsymbol{m}}(\mathbf{0}) \cos (\boldsymbol{\nu}(\boldsymbol{m}) \cdot \boldsymbol{\psi}),
\end{aligned}
$$

y las ecuaciones de movimiento correspondientes son:

$$
\begin{aligned}
& \dot{\psi}_{k}=\frac{\partial H_{\mathcal{C}}}{\partial p_{k}}=\delta_{2, k}\left|\boldsymbol{\omega}^{r}\right|+\frac{p_{1}}{M_{k 1}}+\frac{p_{2}}{M_{k 2}}+\frac{p_{3}}{M_{k 3}} \\
& \dot{p}_{k}=-\frac{\partial H_{\mathcal{C}}}{\partial \psi_{k}}=\epsilon \delta_{1, k} \tilde{V}_{\boldsymbol{m}_{g}}(\mathbf{0}) \sin \left(\psi_{1}\right)+\epsilon \sum_{\boldsymbol{m} \neq \boldsymbol{m}_{g}} \nu_{k}(\boldsymbol{m}) \tilde{V}_{\boldsymbol{m}}(\mathbf{0}) \sin (\boldsymbol{\nu}(\boldsymbol{m}) \cdot \boldsymbol{\psi})
\end{aligned}
$$

El siguiente paso es considerar que es posible apagar todos lo armónicos en la perturbación $V_{P}$, es decir, fijar $\tilde{V}_{\boldsymbol{m}} \equiv 0 \forall \boldsymbol{m} \neq \boldsymbol{m}_{g}$. Entonces se cumple que $p_{2}$ y $p_{3}$ son integrales de movimiento, al igual que cuando $\epsilon$ era nulo. Entonces, es posible elegir condiciones iniciales particulares tales que $p_{2}=p_{3}=0$. Con esta elección, se tiene que $H_{P}$ es otra integral de movimiento y sus ecuaciones para $p_{1}$ y $\psi_{1}$ son independientes de las ecuaciones que involucran a las demás variables (pero no viceversa). Por lo tanto, $p_{1}(t)$ y $\psi_{1}(t)$ constituyen la solución del péndulo simple, y manifestarán régimen de oscilación, rotación, separatriz, o punto de 
equilibrio, dependiendo de la condición inicial. La solución de su separatriz es parecida a la dada en 1.2.7:

$$
\begin{aligned}
& \psi_{s}(t)= \pm 4 \arctan \left(e^{\omega_{0} t}\right) \\
& p_{s}(t)= \pm 2 \sqrt{M \epsilon \tilde{V}_{\boldsymbol{m}_{g}}(\mathbf{0})} \sin \left(\psi_{s}(t) / 2\right) .
\end{aligned}
$$

De la misma puede apreciarse que en el entorno de una resonancia de $\mathcal{O}(\epsilon)$, despreciando el resto de los armónicos en la perturbación, la máxima variación alcanzada por $p_{1}$ en el régimen de libraciones es proporcional a $\sqrt{\epsilon}$. Por lo tanto, considerando (1.89) la máxima variación alcanzada por el vector de acciones no perturbadas $\boldsymbol{I}$ también es proporcional a $\sqrt{\epsilon}$.

La solución para $\boldsymbol{\psi}$ de (1.101), considerando las hipótesis recién mencionadas, es:

$$
\psi_{k}(t)=\delta_{2, k}\left|\boldsymbol{\omega}^{r}\right| t+\frac{M_{11}}{M_{k 1}} \psi_{1}(t)+c_{k} ; \quad k=1,2,3 ;
$$

donde $c_{k}$ son constantes.

Una de las estimaciones cualitativas del desarrollo de Chirikov radica en que la existencia de difusión de Arnold precisa al menos tres resonancias independientes ${ }^{4}$ : una resonancia guía, $\boldsymbol{m}_{g}$, a lo largo de la cual se pretende estudiar la difusión, una resonancia que perturbe a la separatriz de la resonancia guía (produciendo una capa estocástica), llamada layer resonance, y por último, se necesitan una o más resonancias que generen la difusión a lo largo de la resonancia guía, para condiciones iniciales pertenecientes a la capa estocástica. Estas últimas resonancias son llamadas driving resonances. Dado que se está analizando el caso de resonancia de multiplicidad uno, cabe aclarar que en el punto $I^{r}$, la única resonancia existente es la guía. Las otras son resonancias en otros puntos de la superficie de energía. La layer resonance es aquella asociada al término perturbativo trigonométrico más grande de $V_{P}$, mientras que las driving resonances corresponden al resto de los términos trigonométricos.

Aplicando esta teoría, Chirikov (1979) obtiene coeficientes de difusión para dos sistemas particulares. Uno de ellos es el ejemplo de Arnold (1.77) y el otro consiste en dos osciladores cuárticos acoplados.

La teoría de Chirikov no ha sido completamente puesta a prueba aún. Se han realizado experimentos con mapas simplécticos en Chirikov (1979) y en Chirikov et al. (1980), aplicando un método para el cálculo numérico de los coeficientes de difusión basado en promediados temporales múltiples, cuyo propósito es determinar si el proceso es o no difusivo. En Bountis and Kollmann (1994) y Bountis et al. (1998) aplican el método numérico recién mencionado a mapas $4 \mathrm{D}$ que modelan cierta característica de la dinámica de rayos que tiene lugar en aceleradores de partículas. Cincotta (2002) verifica experimentalmente que la hipótesis de estocasticidad reducida para un mapa simpléctico simple no es incondicionalmente válida. En Cachucho et al. (2010) se aplica la teoría de Chirikov al modelar la difusión a lo largo y a través de las capa estocástica de la resonancia $(5,-2,-2)$ de la familia asteroidal (490) Veritas.

En resumen, la teoría de Chirikov se aplica sólamente a resonancias aisladas. Presenta un paradigma básico que sucede en la capa estocástica de la separatriz. Es una teoría cuantitativa, en la que se simplifica (sólo un poco) el tratamiento, mediante el modelado local del sistema con un mapa simpléctico.

\footnotetext{
${ }^{4}$ Es decir, sus vectores asociados deben ser linealmente independientes.
} 


\subsubsection{Pumping estocástico}

Tennyson et al. (1980) proponen un método para estudiar la difusión de Arnold, en particular un método para predecir la difusión que sucede a lo largo de una resonancia perturbada. El punto de partida consiste en analizar que un Hamiltoniano, con sólo un armónico en la perturbación $V$ de Ec. (1.85) no puede presentar difusión de Arnold. Para explicar esto consideremos el Hamiltoniano:

$$
H(\boldsymbol{I}, \boldsymbol{\theta})=H_{0}(\boldsymbol{I})+\epsilon V_{\boldsymbol{m}_{g}}(\boldsymbol{I}) e^{i \boldsymbol{m}_{g} \cdot \theta}+\epsilon V_{\boldsymbol{m}_{l}}(\boldsymbol{I}) e^{i \boldsymbol{m}_{l} \cdot \theta}+\epsilon V_{\boldsymbol{m}_{d}}(\boldsymbol{I}) e^{i \boldsymbol{m}_{d} \cdot \theta},
$$

donde los vectores $\boldsymbol{m}_{g}, \boldsymbol{m}_{l}$ y $\boldsymbol{m}_{d}$ son linealmente independientes. Las ecuaciones de movimiento para las acciones están dadas por:

$$
\frac{d \boldsymbol{I}}{d t}=-i \epsilon\left(V_{\boldsymbol{m}_{g}}(\boldsymbol{I}) e^{i \boldsymbol{m}_{g} \cdot \theta} \boldsymbol{m}_{g}+V_{\boldsymbol{m}_{l}}(\boldsymbol{I}) e^{i \boldsymbol{m}_{l} \cdot \theta} \boldsymbol{m}_{l}+V_{\boldsymbol{m}_{d}}(\boldsymbol{I}) e^{i \boldsymbol{m}_{d} \cdot \theta} \boldsymbol{m}_{d}\right),
$$

de lo que se deduce que si $V_{\boldsymbol{m}_{d}}=0$ entonces el transporte de las acciones esta limitado a la intersección del plano generado por los vectores $\boldsymbol{m}_{g}$ y $\boldsymbol{m}_{l}$, que llamaremos $\Pi$, con la variedad $\mathcal{I}_{\epsilon}$. Otra manera de ver la situación, es notar que para $V_{\boldsymbol{m}_{d}}=0$ es posible elegir nuevas variables canónicas de forma tal que el sistema se desacopla en un sistema con un grado de libertad (por lo tanto integrable) y un sistema con dos grados libertad (en general no integrable). La energía del sistema con $n_{d}=1$ dependerá de las acción original únicamente a través de la componente perpendicular a $\Pi$. Entonces, la difusión en el sistema con $n_{d}=2$ sólo puede adquirir carácter macrosocópico en caso de suceder overlap entre resonancias de la forma $q \boldsymbol{m}_{g}+n \boldsymbol{m}_{l}(q, n \in \mathbb{Z})$ y estará limitada a $\Pi$, como ya fue mencionado. La necesidad de existencia de al menos dos vectores armónicos independientes en la perturbación $V$, para tener difusión de Arnold, coincide con uno de los resultados predichos por la teoría de Chirikov.

Sea el siguiente mapa simpléctico:

$$
\begin{aligned}
\alpha_{n+1} & =\alpha_{n}+2 a_{1} k_{1} \sin k_{1} x_{n}+\epsilon k_{1} 2 \gamma_{n} \\
x_{n+1} & =x_{n}+20 \tan \alpha_{n+1} \\
\beta_{n+1} & =\beta_{n}+2 a_{2} k_{2} \sin k_{2} y_{n}+\epsilon k_{2} 2 \gamma_{n} \\
y_{n+1} & =y_{n}+20 \tan \beta_{n+1},
\end{aligned}
$$

donde $k_{1}, k_{2}, a_{1}, a_{2}$ son números reales y $\gamma_{n} \equiv \sin \left(k_{1} x_{n}+k_{2} y_{n}\right)$. Fijan los valores de algunos parámetros: $a_{1}=a_{2}=2$ y $k_{1}=k_{2}=\pi / 50$.

Para $\epsilon=0$, los movimientos en $[\alpha, x]$ quedan desacoplados de los de $[\beta, y]$. En el plano de fases asociado a $[\beta, y]$, coexisten dos regiones disconexas de comportamiento caótico. Una de ellas es una capa estocástica delgada y la otra es una capa estocástica gruesa.

Cuando $\epsilon \neq 0$, Tennyson et al. (1980) examinan dos procesos difusivos asociados con estas dos capas. Uno de ellos describe la difusión, de la acción $\alpha$, a lo largo de la capa estocástica gruesa del movimiento $[\beta, y]$. La cantidad $\alpha$ experimenta fluctuaciones difusivas originadas debido al pequeño acople con el movimiento aleatorio de $y$. El otro proceso es similar, salvo que ahora $\alpha$ se difunde a lo largo de la fina capa estocástica del movimiento $[\beta, y]$. La región del plano $[\alpha, x]$ que se considerará para las condiciones iniciales será la región de libraciones y por lo tanto regular. La hipótesis fundamental consiste en que el movimiento en $[\beta, y]$ está confinado a la correspondiente capa estocástica. Dicha hipótesis es bastante realista para acoples suficientemente pequeños y mientras el movimiento en el plano $[\alpha, x]$ se mantenga fuera de su propia capa estocástica. Se le llama modelo de stochastic pump debido a que la 
dinámica en la capa (sea fina o gruesa) actúa como una "bomba", transportando energía entre los movimientos $x$ e $y$, en ambos sentidos.

Utilizando dos procedimientos teóricos distintos, según el grosor de la capa, los autores obtienen la expresión del coeficiente de difusión, que en el caso de capa fina, depende del valor de la acción perturbada $(\alpha)$. Posteriormente realizaron una corroboración con experimentos numéricos, verificándose así que el mecanismo de stochastic pump permite modelar la difusión a lo largo de una resonancia en el mapa simpléctico por ellos analizado.

En Easton et al. (2001) se trabaja analíticamente en mapas simplécticos a priori inestables genéricos, que poseen una geometría similar a la del problema de la capa estocástica gruesa recién mencionado. Más específicamente, estudian un mapa 4D, acoplando uno 2D fuertemente caótico a otro 2D casi-integrable. Demuestran teóricamente la existencia de muchas órbitas cuyos momentos se transportan arbitrariamente lejos, incluso cuando el acople es arbitrariamente pequeño. Los autores plantean que con sus resultados no demuestran que este transporte posea características difusivas (en el sentido estocástico).

Kook y Meiss (1990) obtienen analíticamente una aproximación al tensor de difusión para un mapa simpléctico de la forma:

$$
\begin{aligned}
& I_{n+1}=I_{n}-\epsilon \sin \left(\theta_{n}+\psi_{n}\right) \\
& J_{n+1}=J_{n}-K \sin \left(\psi_{n}\right)-\epsilon \sin \left(\theta_{n}+\psi_{n}\right) \\
& \theta_{n+1}=\theta_{n}+I_{n+1} \quad \bmod 2 \pi \\
& \psi_{n+1}=\psi_{n}+J_{n+1} \quad \bmod 2 \pi \text {. }
\end{aligned}
$$

Demuestran que las correlaciones de corto tiempo en el plano $[J, \psi]$ afectan a la difusión en el otro. El tensor de difusión depende de los parámetros del sistema pero no de la acción, $I$. Remarcan que este sistema posee dos límites interesantes. Uno de ellos es el de capa gruesa, para $K>>1$ y $\epsilon<<1$ y el otro es el de capa fina, para $K \lesssim 1$ y $\epsilon<<1$. Realizaron experimentos numéricos para varios valores de los parámetros, encontrando un acuerdo con sus predicciones siempre que $K$ y $\epsilon$ fueran mayores a 2 .

\subsubsection{Teorías de origen estocástico}

Aquí se hará referencia a algunos de los autores que han aplicado el enfoque estocástico (también llamado cinético) al tratamiento de la difusión en problemas deterministas, en particular sistemas Hamiltonianos.

Lichtenberg y Lieberman (1992) deduce una ecuación de este tipo para la variable acción $I$ de un sistema Hamiltoniano 1.5DoF haciendo la aproximación de fase aleatoria. Dicha aproximación es parcialmente válida en regiones con régimen de overlap de resonancias y se basa en la suposición de que las variables ángulo de un sistema Hamiltoniano se mezclan más rápido que lo que lo hacen las variables acción. Varvoglis (2005) aplica la hipótesis de fase aleatoria a un sistema Hamiltoniano 2DoF, obteniendo la correspondiente ecuación de F-P con coeficiente de difusion dependiente de la acción.

Kominis et al. (2010) presenta una jerarquía de ecuaciones para la evolución de la función densidad de probabilidad en el espacio de fases para sistemas Hamiltonianos casi-integrables de dimensión arbitraria, con perturbación dependiente del tiempo. A diferencia de las teorías quasi-lineales (caracterizadas por hipótesis Markoviana, rápida decorrelación de fases, etc), en esta teoría la ecuación cinética posee coeficientes explícitamente dependientes del tiempo, 
aún en el caso en que la perturbación fuera autónoma. Estas ecuaciones sólo han sido probadas mediante experimentos numéricos para un Hamiltoniano integrable 1DoF.

Zaslavsky (2002) revee varios modelos de cinética fraccional y su conexión con modelos dinámicos, topología del espacio de fases y otras características del caos, como son las recurrencias de Poincaré y los dominios sticky. Presenta diversos aspectos de la ecuación cinética fraccional (ECF), incluyendo su derivación, condiciones de aplicabilidad y generalizaciones.

En Denisov et al. (2002) se propone un enfoque cinético al transporte en sistemas Hamiltonianos con espacio de fases mixto. El método se basa en utilizar el formalismo de RW de tiempo contínuo para descomponer la dinámica Hamiltoniana en dos partes alternantes: vuelos balísticos y random walks. La primera de ellas corresponde a la situación en la cual la partícula prueba queda "pegada" a los complejos bordes de las islas de estabilidad. Durante esta etapa, la partícula se comporta determinísticamente, moviéndose aproximadamente a una velocidad constante para cada isla. La duración del vuelo está completamente determinada por una función densidad de probabilidad, particular en cada isla. La otra parte modela con movimiento Browniano a las porciones de trayectoria que tienen lugar en la componente caótica, y que suceden entre dos vuelos consecutivos. También se utiliza una función densidad de probabilidad para modelar el tiempo que la partícula permanece haciendo RW. El enfoque descansa sobre la hipóthesis de que existan movimientos suficientemente largos en la región caótica como para que las correlaciones entre vuelos consecutivos sean despreciables.

Aplican el método a un Hamiltoniano 1.5DoF y estiman el comportamiento de la desviación media cuadrática adentro de la capa caótica del mismo. Obtienen un comportamiento superdifusivo para la coordenada espacial: $\left\langle x^{2}(t)>\propto t^{w}\right.$, con $w \approx 1.6$ y lo verifican con un experimento numérico, utilizando un ensamble de $10^{4}$ trayectorias.

Venegeroles (2008) estudia el fenómento de superdifusión, presente en un mapa simpléctico 2D, debido a los llamados accelerator modes. Computa analíticamente el coeficiente de superdifusión y su correspondiente exponente de transporte, partiendo de primeros principios y comparando contra el coficiente de difusión quasi-lineal. Verifica sus predicciones numéricamente.

Čubrović (2005) propone un modelo cinético de transporte en espacio de acciones de sistemas Hamiltonianos no integrables. El mismo está basado en una ecuación cinética fraccional con un coeficiente de difusión no homogéneo. Una particularidad de este método es que considera a la acción como un escalar y no considera la difusión de Arnold. Presenta evidencia numérica que valida su modelo.

Klafter y Zumofen (1994) estudia la difusión en un sistema Hamiltoniano con una componente no integrable de gran tamaño, haciendo una análisis estadístico en el marco de un proceso Lévy walk.

\subsection{Modelos de difusión en astronomía}

La astronomía y la física de aceleradores de partículas, han sido las principales impulsoras del desarrollo de la dinámica, en general, y de las teorías de difusión en sistemas Hamiltonianos, en particular.

Dentro de la astronomía, el sistema sobre el que se han realizado la mayoría de las apliaciones de teorías difusivas es el Sistema Solar, debido principalmente a la posibilidad de contar con datos observados de calidad.

El Hamiltoniano del problema gravitatorio de dos cuerpos (problema Kepleriano) posee 
solución analítica exacta. Tal solución puede expresarse en función de seis elementos orbitales $a, e, i, \omega, \Omega$ y $E$ que representan, respectivamente, el semieje mayor, la excentricidad, la inclinación del plano orbital respecto a algún plano de referencia, el argumento del pericentro, la longitud del nodo y la anomalía excéntrica. Los primeros cinco elementos orbitales son constantes de movimiento del problema Kepleriano.

Por otro lado, al modelar al Sistema Solar dinámicamente, considerando más de dos cuerpos atraídos por la fuerza gravitatoria, el sistema Hamiltoniano obtenido no es integrable. Dependiendo de los cuerpos bajo estudio y de las condiciones iniciales elegidas, dicho Hamiltoniano puede ser (o no) considerado como casi-integrable y analizado bajo el enfoque de la teoría perturbativa. En la misma, el Hamiltoniano integrable puede estar conformado por el problema gravitatorio de dos cuerpos (problema Kepleriano). Dicho Hamiltoniano presenta degeneración esencial y K-degeneración, su superficie de energía $\left(\mathcal{I}_{0}\right)$ no es convexa, y el espacio de fases no es acotado. Estas características hacen que este problema quede afuera de las hipótesis necesarias para las teorías de la difusión mencionadas en la sección anterior. Sin embargo, existen trabajos cuyo objetivo ha sido el lograr generalizaciones a dichas teorías capaces de ser aplicadas en el problema de dos cuerpos perturbado. En estos casos, en general, se estudia el proceso difusivo de cantidades asociadas a $a, e, i$, llamadas valores propios, para distintas configuraciones de los cuerpos y en distintas regiones del Sistema Solar, que por lo general son entornos de resonancias. Por citar algunos se menciona a Robutel et al. (2005), Robutel and Gabern (2006), Tsiganis et al. (2007) y Cachucho et al. (2010). Debido a la relación metodológica de esta tesis, en lo que respecta a cómputo de coeficientes de difusión a través de varianzas de ensamble, con los trabajos Cordeiro y Mendes de Souza (2005) y Cordeiro (2006), se resume el primero de ellos a continuación.

En dicho estudio se presenta evidencia numérica de que para describir los procesos difusivos que tienen lugar en regiones de resonancias Jovianas de primer orden, es más adecuado utilizar un modelo RW con difusión anómala que utilizar uno con difusión normal. En este trabajo se utilizan ensambles de partículas prueba para determinar la evolucion temporal de la desviación estándar del semieje mayor $\left(\sigma_{a}\right)$ y de la excentricidad $\left(\sigma_{e}\right)$. Cada una de estas curvas presenta oscilaciones de orden de magnitud relativo cercano a uno. Sin embargo, es posible apreciar que la envolvente de estas cantidades obedece, aproximadamente, una ley tipo MBF (1.74). Posteriormente proponen un modelo probabilístico para dicho comportamiento, ajustan el valor del exponente de Hurst $(v)$ y conforman mapas cromáticos de tal cantidad sobre el plano $a, e$, en entornos de las resonancias Jovianas 2:1, 3:2 y 4:3. Se debe aclarar que bajo el término "ensamble", los autores se refieren a un quasi-ensamble de partículas de prueba con condiciones iniciales quasi-idénticas. La elección de este método de ensambles está justificado por la siguiente propiedad: los períodos seculares no afectan a la evolución de la desviación estándar porque los cambios principales experimentados por una partícula de un ensamble son también experimentados por todas las otras partículas del ensamble. Esto permite adoptar intervalos de integración que son más pequeños que los períodos seculares típicos de $a$ y $e$ en las regiones resonantes estudiadas por ellos. Este método de ensambles será adoptado para el cálculo de cantidades estadísticas en capítulos 4 y 5 .

Los autores concluyen que en los bordes de resonancias (capas estocásticas), la difusión se comporta exponencialmente, es decir, $\sigma(t)=\alpha e^{\beta t^{\gamma}}$ ( $\alpha, \beta$ y $\gamma$ constantes) al inicio pero luego prosigue una lenta difusión con un crecimiento de las desviaciones estándar despreciable. También encuentran que en la región resonante, la desviación estándar no muestra inicialmente un comportamiento difusivo. Sin embargo, habiendo transcurrido un cierto intervalo de tiempo, la difusión actúa como difusión anómala asociada a una ley de potencias. Además, 
presentan un análisis de la correlación entre las regiones pobladas por asteroides y los valores de $v$ asociados.

En el capítulo 4 se medirá la difusión en los bordes de una resonancia de un sistema Hamiltoniano 3DoF, focalizando en la etapa de difusión lenta mencionada en párrafo anterior. $\mathrm{Al}$ integrar durante tiempos relativamente mayores, se podrá observar un aumento neto de la varianza y cuantificar su razón de cambio.

Otros sistemas astronómicos donde los efectos de la difusión caótica han sido estudiados son los modelos de sistemas extrasolares y las galaxias. En Efthymiopoulos et al. (2007) se analizan diversos aspectos de la dinámica galáctica, haciendo la conexión con el problema de la difusión en sistemas Hamiltonianos. En particular, se menciona que es de suma importancia para la dinámica galáctica comprender el rol de la difusión de Arnold y de la difusión en régimen de overlap. Asimismo se brindan ejemplos de difusión de órbitas debilmente caóticas a lo largo de lineas de resonancia en el espacio de frecuencias. En Efthymiopoulos (2010) se continua el estudio precedente, demostrando como la teoría perturbativa resonante lleva a una teoría orbital para la estructura de galaxias espirales.

\subsection{Objetivos}

El objetivo de esta tesis es avanzar en el conocimiento de los procesos difusivos que tienen lugar en el espacio de acciones no perturbadas de sistemas Hamiltonianos casi-integrables. En particular, se estudia la difusión de Arnold que tiene lugar a lo largo de una resonancia aislada, debido a los términos perturbativos de orden superior. Para ello se estudiarán dos sistemas dinámicos autónomos.

Uno de ellos es un flujo Hamiltoniano 3DoF casi-integrable cuya superficie de energía no perturbada $\left(\mathcal{I}_{0}\right)$ es convexa. En el capítulo 3 se determina el valor de $\epsilon_{c}$ que separa los regímenes de Nekhoroshev y de Chirikov, mediante la utilización del criterio de overlap. En el capítulo 4 se realizan experimentos numéricos de difusión para distintos valores de $\epsilon$. En los mismos se computará la evolución temporal de varianzas y desplazamientos medios cuadráticos asociados a ensambles de partículas de prueba, en las tres direcciones de la base de Chirikov, pero focalizando en aquella que mide la difusión de Arnold. Bajo la hipótesis de que las varianzas se comportan tipo ley de potencias, se harán ajustes de los correspondientes exponentes de Hurst, obteniendo comportamientos subdifusivos. Además, en intervalos temporales suficientemente cortos, como para observar comportamiento aproximadamente lineal (de la varianza), se calcularán los coeficientes de difusión, $\mathcal{D}_{\sigma}(\epsilon)$.

Asimismo, se hará un experimento fijando el valor de $\epsilon$ en uno correspondiente al régimen de Nekhoroshev, para ensambles de condiciones iniciales situados a la largo de la capa estocástica de una de las resonancias del sistema. En esta experiencia se podrá observar el efecto producido en la difusión por otras resonancias cercanas y se dará evidencia de una componente anómala en la difusión de algunos de los ensambles.

En el capítulo 5 se aplica empíricamente un método, desarrollado para sistemas estocásticos, a un mapa simpléctico 4D a priori inestable. Si bien este mapa no es casi-integrable, se estudia con objeto de poder controlar en forma independiente el grosor de la capa estocástica y el nivel de acople entre la misma y la acción, mediante los parámetros $K$ y $\epsilon$, respectivamente. Este mapa es similar al utilizado en el modelo de pumping estocástico y es igual al dado por la Ec. (1.107). A diferencia de lo obtenido por Kook y Meiss (1990), en el resultado de esta tesis el coeficiente de difusión asociado a la dirección de Arnold sí depende de la acción. 
Además, se verificará con experimentos numéricos, que la evolución de la función densidad de probabilidad de la mencionada acción satisface aproximadamente una ecuación de F-P. En dicho capítulo se muestra la concordancia entre predicción y medición para los valores $K=3$ (caso capa gruesa) y $K=0.9$ (caso capa fina), tanto en lo que respecta al coeficiente de difusión como a la solución de la mencionada ecuación cinética. 


\section{Indicadores de caos}

En el capítulo anterior se habló de caos. En este capítulo presentaremos una definición pragmática de dicho término y mostraremos algunas maneras de medirlo o identificarlo. Se introducirán algunos indicadores dinámicos utilizados para visualizar diversas secciones del espacio de fases. En los capítulos subsiguientes, esta herramienta nos permitirá ubicar resonancias y, en particular, sus capas estocásticas (o separatrices perturbadas). Esto será una tarea imprescindible para poder elegir con precisión ensambles de partículas de prueba dentro de las capas estocásticas.

Los indicadores utilizados pertenecen a la familia de los indicadores variacionales ya que se basan en el análisis del comportamiento de la solución de las ecuaciones variacionales del sistema. Estos son el Exponente Característico de Lyapunov (ECL) a tiempo finito, el FLI, el MEGNO y el SALI. Maffione et al. (2011) realiza una comparación entre estos y otros indicadores para el caso de mapas. En los últimos años han aparecido varios tipos de indicadores que intentan mejorar distintos aspectos, entre ellos la rapidez.

Para sistemas dinámicos de tiempo continuo (1.2) las correspondientes ecuaciones variacionales están dadas por:

$$
\frac{d}{d t} \mathbf{v}_{t}=\mathbf{D f}\left(\mathbf{r}_{t}\right) \cdot \mathbf{v}_{t},
$$

donde $\mathbf{D f}(\mathbf{r})$ es la matriz Jacobiana (1.4) de la función $\mathbf{f}$ y $\mathbf{v}_{t}$ es un vector desviación al tiempo $t$.

Análogamente para mapas (1.1), el correspondiente sistema ecuaciones variacionales a tiempo discreto, también llamado dinámica de mapa tangente, asociado a una dada órbita $\left\{\mathbf{r}_{t}\right\}_{t \in \mathbb{N}}$ es el siguiente:

$$
\mathbf{v}_{t+1}=\mathbf{D f}\left(\mathbf{r}_{t}\right) \cdot \mathbf{v}_{t} .
$$

\subsection{Exponentes Característicos de Lyapunov}

Skokos (2010) hace una revisión histórica de la definición de los ECL y habla sobre su conexión con la divergencia de órbitas cercanas. Oseledec (1968) establece el teorema que garantiza la existencia (salvo en un subconjunto del espacio de fases con medida nula) de un espectro de exponentes característicos de Lyapunov y, en particular, la actual definición del 
máximo ECL (mECL) en términos de la solución de las ecuaciones variacionales:

$$
\operatorname{mECL} \equiv \lim _{t \rightarrow \infty} \frac{1}{t} \ln \frac{\left\|\mathbf{v}_{t}\right\|}{\left\|\mathbf{v}_{0}\right\|},
$$

con $\|$.$\| alguna norma en el espacio de fases. En este capítulo se adoptará la adoptará la$ norma Euclideana.

Se define como órbita caótica a toda órbita cuyo mECL es positivo y esto implica la existencia de divergencia exponencial de órbitas cercanas. Por otro lado, una órbita regular es aquella con mECL igual a cero.

De forma tal de tener una cantidad computable numericamente definimos el mECL al tiempo finito $t$ como

$$
\operatorname{mECL}(t) \equiv \frac{1}{t} \ln \frac{\left\|\mathbf{v}_{t}\right\|}{\left\|\mathbf{v}_{0}\right\|},
$$

por lo que la Ecuación (2.3) puede ser reformulada así:

$$
\mathrm{mECL}=\lim _{t \rightarrow \infty} \operatorname{mECL}(t) .
$$

En la práctica, para evitar saturación numérica debido al crecimiento exponecial de los módulos $\left\|\mathbf{v}_{t}\right\|$, se acostumbra a utilizar la siguiente expresión para el el ECL de tiempo finito:

$$
\operatorname{mECL}(t)=\frac{1}{t} \sum_{k=1}^{t} \ln \frac{\left\|\mathbf{v}_{k}\right\|}{\left\|\mathbf{v}_{k-1}\right\|} .
$$

\section{2. $\quad$ Smaller Alignment Index}

El SALI, introducido por Skokos (2001), mide el grado con el cual un par de vectores inicialmente linealmente independientes tiende a alinearse. El principio subyacente es que, para órbitas caóticas, un vector desviación bajo la dinámica de las ecuaciones variacionales cambia de forma tal de volverse alineado con la dirección instantánea más inestable. En otras palabras, para casi todo par de vectores desviación iniciales $\left(\mathbf{v}_{0}, \mathbf{u}_{0}\right)$, mientras mas caótica sea la órbita, más rápido se reducirá a cero el ángulo entre ellos.

El SALI es definido como:

$$
\operatorname{SALI}(t)=\min \left\{\left\|\frac{\mathbf{v}_{\mathbf{t}}}{\left\|\mathbf{v}_{\mathbf{t}}\right\|}+\frac{\mathbf{u}_{\mathbf{t}}}{\left\|\mathbf{u}_{\mathbf{t}}\right\|}\right\|,\left\|\frac{\mathbf{v}_{\mathbf{t}}}{\left\|\mathbf{v}_{\mathbf{t}}\right\|}-\frac{\mathbf{u}_{\mathbf{t}}}{\left\|\mathbf{u}_{\mathbf{t}}\right\|}\right\|\right\}
$$

Para controlar el aumento exponencial de la norma de vectores y evitar problemas de overflow, Skokos et al. (2004) los han normalizado en cada paso temporal. Así definido, se cumple que $\operatorname{SALI}(t) \in[0, \sqrt{2}]$ y que SALI $=0$ sí y sólo sí ambos vectores normalizados poseen la misma dirección, siendo iguales u opuestos.

Christodoulidi and Bountis (2006) expone los distintos comportamientos temporales que puede presentar el indicador, resumidos a continuación, para sistemas Hamiltonianos con $n_{d}$ grados de libertad. Para órbitas caóticas, y $t \gg 1$, se cumple que

$$
\operatorname{SALI}(t) \sim e^{-\left(\lambda_{2}-\lambda_{1}\right) t},
$$

donde $\lambda_{1} \equiv$ mECL y $\lambda_{2}\left(<\lambda_{1}\right)$ es el ECL que le sigue en magnitud a $\lambda_{1}$. 
Para órbitas regulares, el comportamiento depende de la dimensión del toro, denotada $m$, donde $1 \leq m \leq n_{d}$. Asintóticamente, para $t \gg 1$, se tiene que:

$$
\operatorname{SALI}(\mathrm{t}) \sim \begin{cases}\text { constante } & \text { si } 2 \leq m, \\ \frac{1}{t^{2-m}} & \text { si } m<2 \leq 2 n_{d}-m, \\ \frac{1}{t^{2\left(2-n_{d}\right)}} & \text { si } 2 n_{d}-m<2 \leq 2 n_{d}\end{cases}
$$

Observando esta última ecuación se tiene que hay situaciones de órbitas regulares en las que el SALI converge a cero, al igual que en el caso caótico. Sin embargo, la diferencia en la velocidad de convergencia es lo que permite determinar si un movimiento es regular o caótico. En el primer caso, la convergencia a cero, de existir, obedece una ley de potencias, mientras que en el otro caso la convergencia a cero es exponencialmente rápida. Ver (Skokos et al., 2004) y (Skokos et al., 2007) para un test numérico del SALI y su generalización, la familia GALI.

\section{3. $\quad$ Fast Lyapunov Indicator}

Desde su presentación en Froeschlé et al. (1997b), el Fast Lyapunov Indicator (FLI) ha sido levemente modificado en algunas oportunidades de forma tal de responder a diferentes preguntas.

Sin embargo, la esencia del indicador básicamente se ha mantenido. Originalmente, fue concebido (Froeschlé et al., 1997a) como el supremo de las normas de una base de vectores desviación en evolución. Tal base se toma inicialmente unitaria. Un poco más tarde, esta definición fue subsituida en Froeschlé y Lega (2000) por una definición computacionalmente más económica que requería la evolución de sólo un vector desviación $(\mathbf{v})$ de longitud $\delta(t) \equiv$ $\|\mathbf{v}(t)\|:$

$$
\operatorname{FLI}(t) \equiv \ln \|\mathbf{v}(t)\|=\ln \delta(t) .
$$

Esta expresión ha sido usada para obtener resultados analíticos por Froeschlé y Lega (2000) and Guzzo et al. (2002).

Dentro de este capítulo trabajaremos con esta última definición, siendo la misma tanto para flujos Hamiltonianos como para mapas simplécticos. Para una futura utilización, mantendremos en mente que el promedio temporal del FLI en el intervalo $[0, t]$ esta, respectivamente para flujos y mapas, dado por

$$
\begin{aligned}
& \overline{\operatorname{FLI}}(t) \equiv \frac{1}{t} \int_{0}^{t} \operatorname{FLI}(s) d s=\frac{1}{t} \int_{0}^{t} \ln \delta(s) d s, \quad \mathrm{y} \\
& \overline{\operatorname{FLI}}(t) \equiv \frac{1}{t} \sum_{k=0}^{t} \operatorname{FLI}(k)=\frac{1}{t} \sum_{k=0}^{t} \ln \delta(k) .
\end{aligned}
$$

\subsection{Mean Exponential Growth Factor of Nearby Orbits}

El Mean Exponential Growth Factor of Nearby Orbits (MEGNO) fue introducido por primera vez por Cincotta and Simó (2000), para sistemas de tiempo continuo, como:

$$
Y(t) \equiv \frac{2}{t} \int_{0}^{t} \frac{\dot{\delta}(s)}{\delta(s)} s d s
$$


Es el doble del promedio pesado con el tiempo de la divergencia relativa de órbitas. Uno de los objetivos de su invención fue permitir una clara clasificación entre órbitas caóticas, regulares KAM y regulares resonantes. Su versión para tiempo discreto apareció en Cincotta et al. (2003) de acuerdo con la siguiente expresión:

$$
Y(t) \equiv \frac{2}{t} \sum_{k=1}^{t} k \ln \left(\frac{\delta(k)}{\delta(k-1)}\right)
$$

Cincotta et al. (2003) define una familia biparamétrica de indicadores MEGNO:

$$
Y_{m, j}(n)=(m+1) n^{j} \sum_{k=1}^{n} k^{m} \ln \frac{\left\|\mathbf{v}_{k}\right\|}{\left\|\mathbf{v}_{k-1}\right\|},
$$

donde $m$ y $j$ son números enteros. Ellos hicieron experimentos con $Y_{2,0}, Y_{3,1}$ y $Y_{1,-1}$ cocluyendo que el último permite tanto una rápida clasificación entre órbitas caóticas y regulares, como una clara identificación de órbitas periódicas estables e inestables. Debido a este hecho, usaremos $Y \equiv Y_{1,-1}$ durante todo este trabajo. Para reducir las rápidas oscilaciones que la evolución temporal del MEGNO presenta, en Cincotta et al. (2003) ellos utilizaron un promedio temporal cumulativo móvil de esta cantidad:

$$
\bar{Y}(n)=\frac{1}{n} \sum_{k=1}^{n} Y(k)
$$

Teóricamente, la evolución asintótica de $\bar{Y}(n)$ para cualquier régimen dinámico puede ser expresada como

$$
\bar{Y}(n) \approx \frac{\mathrm{mECL}}{2} n+c,
$$

donde $c \approx 0$ y 2 para movimiento caótico y regular, respectivamente.

\subsection{La relación entre FLI y MEGNO}

Ahora demostraremos una relación analítica exacta entre el FLI y el MEGNO, que ha sido publicada en Mestre et al. (2011). Empecemos analizando el caso de flujo Hamiltoniano mediante la reescritura de (2.13) en esta forma:

$$
Y(t)=\frac{2}{t} \int_{0}^{t} s \frac{1}{\delta(s)} \frac{d \delta(s)}{d s} d s=\frac{2}{t} \int_{0}^{t} s \frac{d}{d s}[\ln \delta(s)] d s .
$$

Integrando por partes eligiendo $u(s)=s$ y $d v(s)=\frac{d}{d s}[\ln (\delta(s))] d s$, tenemos que $d u(s)=d s$ y $v(s)=\ln (\delta(s))$, por lo cual:

$$
\begin{aligned}
Y(t) & =\frac{2}{t}\left\{\left.u(s) v(s)\right|_{0} ^{t}-\int_{0}^{t} v(s) d u(s)\right\} \\
& =2\left\{\ln \delta(t)-\frac{1}{t} \int_{0}^{t} \ln \delta(s) d s\right\}
\end{aligned}
$$


donde hemos usado que $\delta(0)=1$. Comparando esta ecuación con (2.10) y (2.11) llegamos al hecho de que el MEGNO es dos veces la diferencia entre el FLI y su promedio temporal en $[0, t]$ :

$$
Y(t)=2\{\operatorname{FLI}(t)-\overline{\operatorname{FLI}}(t)\}
$$

Este resultado permite entender dos hechos que han sido recientemente mencionados en la literatura.

Un punto es que el criterio del MEGNO toma ventaja de la información dinámica de la evolución del vector tangente a lo largo de la órbita completa, según Valk et al. (2009) y Hinse et al. (2010). La Ec. (2.21) nos dice exactamente en que forma el indicador incluye esta información; en cada paso temporal el MEGNO substrae del FLI el valor promedio de este último.

El otro punto a discutir, explicitamente mencionado en Breiter et al. (2008) y Barrio et al. (2009), es la razón por la cual el MEGNO da el grado de caoticidad en una escala absoluta mientras que el FLI sólo da valores relativos. Es decir, en el caso de órbitas regulares el MEGNO tiende asintóticamente hacia un valor constante (dos) mientras que el FLI se comporta logarítmicamente, no permitiendo tener un criterio independiente del tiempo para establecer el límite que divide órbitas caóticas de regulares. Esto es el motivo por el cual si queremos decidir si una órbita es o no caótica, a un tiempo fijo $t$, aplicando el criterio FLI, debemos primero hacer pruebas de su comportamiento en órbitas con carácter regular previamente establecido.

Para explicar la situación con un ejemplo analicemos primero el caso de una órbita regular KAM ideal. Debido a la bien conocida rotación diferencial que sucede en sistemas integrables no lineales, hacemos al aproximación de que para movimiento ordenado en órbitas KAM las normas de los vectores desviación satisfacen $\delta(t) \approx 1+\beta t$ ( $\beta \neq 0$ una constante). En este caso tenemos que:

$$
\operatorname{FLI}(t) \approx \ln (1+\beta t)
$$

$\mathrm{y}$

$$
\overline{\mathrm{FLI}}(t) \approx \ln (1+\beta t)+\frac{\ln (1+\beta t)}{\beta t}-1 .
$$

Por lo tanto, considerando (2.21):

$$
Y(t) \approx 2\left\{1-\frac{\ln (1+\beta t)}{\beta t}\right\}
$$

con lo que redescubrimos el ya mencionado límite asintótico del MEGNO para movimiento regular.

Por otro lado, en el caso de una órbita caótica, con $\delta(t) \approx e^{\lambda_{1} t}$, es posible utilizar la relación MEGNO-FLI para demostrar que ambos indicadores se comportan similarmente. De hecho, ambos se comportan linealmente con el tiempo con una pendiente igual a $\lambda_{1}$.

En el caso de mapas simplécticos, la relación entre el MEGNO y el FLI es levemente diferente. Empezando por (2.14), se tiene el siguiente desarrollo: 


$$
\begin{aligned}
Y(t) & =\frac{2}{t}\left\{\sum_{k=1}^{t} k \ln \delta(k)-\sum_{k=1}^{t} k \ln \delta(k-1)\right\} \\
& =\frac{2}{t}\left\{\sum_{k=1}^{t} k \ln \delta(k)-\sum_{k=0}^{t-1}(k+1) \ln \delta(k)\right\} \\
& =2\left\{\ln \delta(t)-\frac{1}{t} \sum_{k=0}^{t} \ln \delta(k)+\frac{1}{t} \ln \delta(t)\right\} .
\end{aligned}
$$

Luego, comparando con (2.10) y (2.12), llegamos a que

$$
Y(t)=2\{\operatorname{FLI}(t)-\overline{\mathrm{FLI}}(t)\}+(2 / t) \ln \delta(t),
$$

y como el término a la derecha tiende asintóticamente a $2 \lambda_{1}$, hemos demostrado que, para mapas, el MEGNO y el FLI satisfacen la Ec. (2.21) con un error de orden $\lambda_{1}$ :

$$
Y(t)=2\{\operatorname{FLI}(t)-\overline{\operatorname{FLI}}(t)\}+\mathcal{O}\left(\lambda_{1}\right) .
$$

Se remarca que en ambas situaciones, regular y caótica, el valor de $\lambda_{1}$ es despreciable respecto a los otro términos, como mostraremos con experimentos numéricos a continuación.

\subsubsection{Experimentos con el Mapa Standard.}

En estos experimentos revisaremos la validez de (2.27) tanto para movimiento ordenado como desordenado, trabajando con el Mapa Estándar (Standard Map, SM), donde la evolución temporal de las dos variables $I$ y $\theta$ está determinada por:

$$
\begin{aligned}
& I_{t+1}=I_{t}+K \sin \theta_{t} \\
& \theta_{t+1}=\theta_{t}+I_{t+1} \quad \bmod 2 \pi \text {. }
\end{aligned}
$$

Dicho mapa fue introducido en Chirikov (1979) y ha sido extensamente estudiado en la literatura. Entre sus características se menciona que existe un valor crítico del parámetro: $K_{c} \approx 0.97$ (Greene, 1979 ) en el cual el último toro KAM invariante ha sido destruído. Para $K \lesssim K_{c}$ se está en el régimen de capa delgada y para $K \gtrsim K_{c}$ se está en régimen de capa gruesa (overlap). En particular, si $K \lesssim 4$ aún se mantienen estables los puntos fijos de las resonancias enteras mientras que si $K \gtrsim 4$, éstos se tornan inestables y prácticamente todo el espacio de fases del SM es caótico.

En el siguiente experimento se ha trabajado con un parámetro $K=0.90$, En la Fig. 2.1 mostramos, para $t \in[0,2000]$, una órbita caótica y una regular en puntos rojos y azules, respectivamente. La trayectoria caótica pertenece a la capa estocástica (separatriz perturbada), con condición inicial $\left(I_{0}, \theta_{0}\right)=(\pi / 100,0.628)$, mientras que la regular está en el régimen oscilatorio, con condición inicial $\left(I_{0}, \theta_{0}\right)=(\pi / 100,1.884)$. En la Fig. 2.2 desplegamos, para la primera órbita, los valores de $\operatorname{FLI}(t), \overline{\mathrm{FLI}}(t), Y(t) / 2$ y $\operatorname{FLI}(t)-\overline{\mathrm{FLI}}(t)$, en colores rojo, azul, negro y amarillo, respectivamente. Existe buen acuerdo con la Ec. (2.27), incluso desde muy temprano. En la Fig. 2.3 podemos ver la evolución de las mismas cantidades, en los mismos colores, para la otra órbita. En este caso es mas evidente la diferencia inicial entre $Y(t) / 2$ y $\operatorname{FLI}(t)-\overline{\operatorname{FLI}}(t)$, para tiempos más cortos que cien unidades de tiempo. 


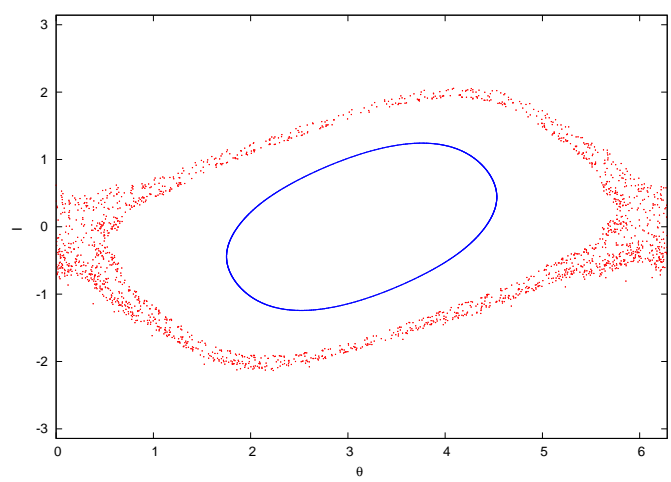

Figura 2.1: Una órbita caótica (rojo) y una regular (azul) del Mapa Standard, con $K=0.90$.

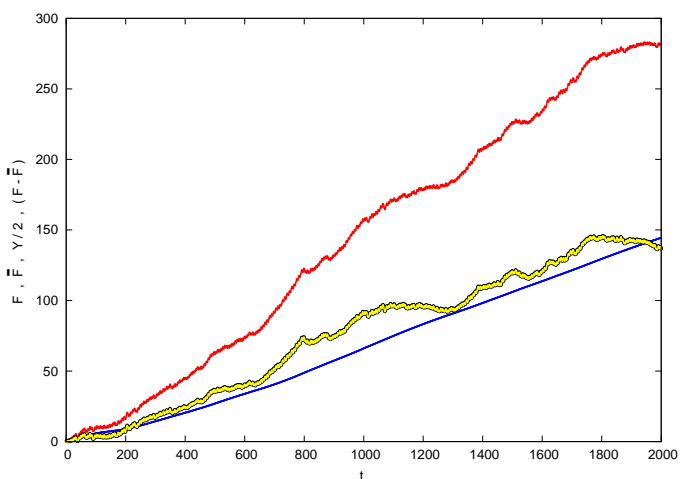

Figura 2.2: Valores de $\operatorname{FLI}(t)$ (rojo), $\overline{\operatorname{FLI}}(t)$ (azul), $Y(t) / 2$ (negro) y $\operatorname{FLI}(t)-\overline{\operatorname{FLI}}(t)$ (amarillo) para una órbita caótica del SM $(K=0.90)$. En la etiqueta del eje vertical, se ha aplicado la abreviación $\mathrm{F} \equiv$ FLI.

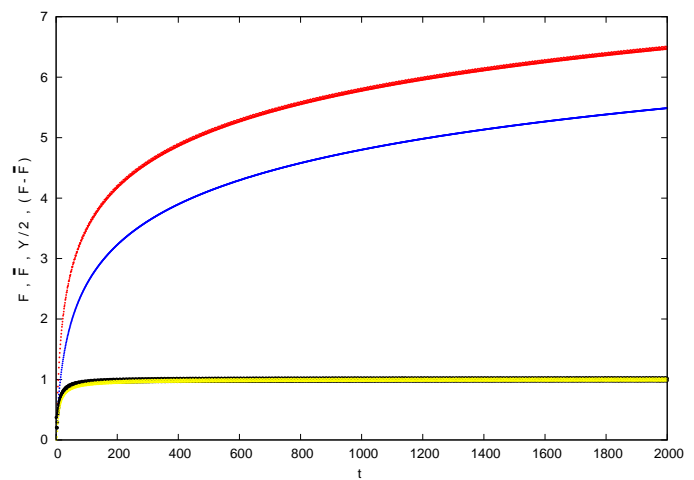

Figura 2.3: Valores de $\operatorname{FLI}(t)$ (rojo), $\overline{\operatorname{FLI}}(t)$ (azul), $Y(t) / 2$ (negro) y $\operatorname{FLI}(t)-\overline{\operatorname{FLI}}(t)$ (amarillo) para una órbita regular del SM $(K=0.90)$. En la etiqueta del eje vertical, se ha aplicado la abreviación F $\equiv$ FLI. 


\subsection{Visualización de estructura resonante}

En esta sección se muestra cómo los indicadores arriba mencionados constituyen una herramienta para visualizar la estructura resonante en sistemas Hamiltonianos, aplicando algunos de ellos a un mapa 4D no integrable. El trabajo presentado en esta sección será publicado en Faranda et al. (2012). Como ejemplo consideramos un mapa simpléctico casiintegrable extensamente utilizado en la literatura (Guzzo et al., 2005). Este mapa está definido así:

$$
\begin{aligned}
\theta_{n+1} & =\theta_{n}+I_{n} \quad \bmod 2 \pi \\
\phi_{n+1} & =\phi_{n}+J_{n} \quad \bmod 2 \pi \\
I_{n+1} & =I_{n}-\mu \frac{\partial V\left(\theta_{n+1}, \phi_{n+1}\right)}{\partial \theta_{n+1}} \\
J_{n+1} & =J_{n}-\mu \frac{\partial V\left(\theta_{n+1}, \phi_{n+1}\right)}{\partial \phi_{n+1}} ;
\end{aligned}
$$

donde $V \equiv 1 /(\cos (\theta)+\cos (\phi)+2+c)$, con $c>0$ y $\mu$ es el parámetro perturbativo. Hemos tomado una grilla de $1146 \times 1146$ condiciones iniciales con acciones $(I, J) \in[0,3.6] \times[0,3.6]$ y un par de ángulos fijos, $(\theta, \phi)=(0.5,0.5)$, y computado $\operatorname{mECL}(t), \bar{Y}(t)$ y $\operatorname{SALI}(t)$ para $t=10^{3}$. Asociando estos valores con cada condición inicial y utilizando una escala cromática hemos realizado la Figura 2.4, donde los valores de los parámetros utilizados son $c=2 \mathrm{y}$ $\mu=0.6$. La imagen superior izquierda corresponde al mECL, la superior derecha a $\bar{Y}$ y la inferior al SALI. En el caso del mECL y del SALI, se ha utilizado el logaritmo natural del valor absoluto del correspondiente indicador. Debido al hecho de que este último indicador converge a cero exponencialmente rápido para órbitas caóticas, se ha utilizado un valor de corte en SALI $=10^{-16}$. La red de Arnold es visible por cada indicador y su estructura es la misma. En particular, todos detectan las capas estocásticas de los modelos de péndulos asociados las resonancias y detectan la complejidad de la estructura resonante en los entornos de las intersecciones de resonancias. Además, valores más altos del mECL se encuentran sobre la diagonal $I=J$, lo cual es coherente con el hecho de que sobre la diagonal se encuentra la mayor inestabilidad.

\subsection{Conclusión}

En este capítulo se han mencionado algunos indicadores dinámicos variacionales y se ha demostrado una fuerte relación analítica entre dos de ellos, el MEGNO y el FLI. Además, se han estudiado implicaciones de esta conexión y presentado experimentos numéricos que soportan nuestras estimaciones teóricas. Finalmente se ha mostrado que estos indicadores permiten trazar la estructura resonante de un sistema Hamiltoniano, así como ubicar las capas estocásticas asociadas. 

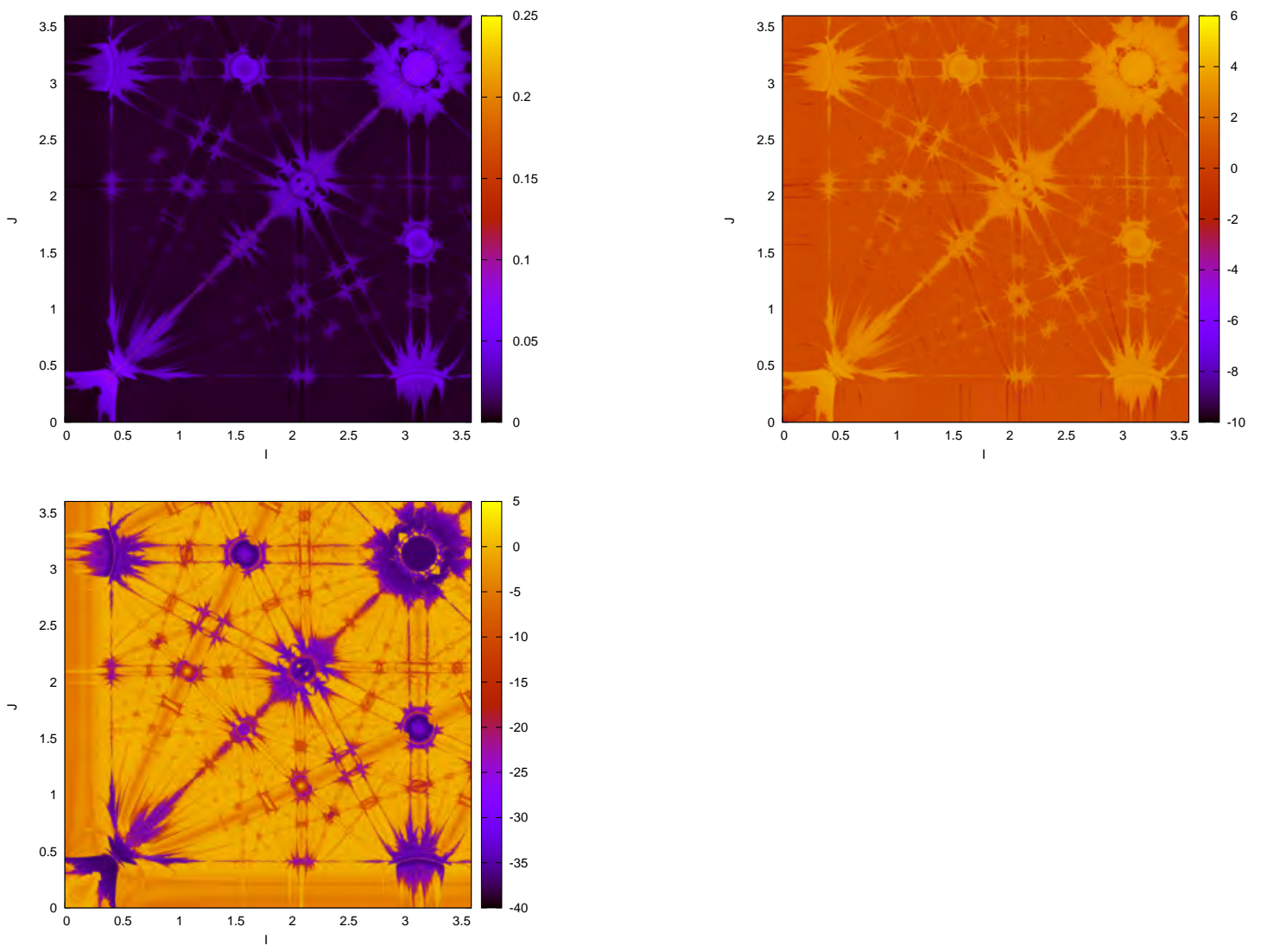

Figura 2.4: Estructura resonante en el espacio de acciones de mapa simpléctico 4D, determinada mediante mapas cromáticos de los indicadores $\operatorname{mECL}\left(10^{3}\right), \bar{Y}\left(10^{3}\right)$, y $\operatorname{SALI}\left(10^{3}\right)$, en las ubicaciones superior izquierda, superior derecha e inferior, respectivamente. 


\section{La estructura resonante del flujo Hamiltoniano y búsqueda del $\epsilon_{c}$.}

En la subsección 1.4.4 se analizó el motivo por el cual el ancho de una resonancia a $\mathcal{O}(\epsilon)$ es proporcional a $\sqrt{\epsilon}$ para perturbaciones suficientemente pequeñas. A su vez, en 1.2.8 se explicó por qué la separatriz se transforma en una capa estocástica al ser perturbada.

Consideremos un caso ideal de sólo dos resonancias en el espacio de acciones de un sistema de 2DoF. Sean $\boldsymbol{I}_{a}^{r}$ y $\boldsymbol{I}_{b}^{r}$ las correspondientes acciones resonantes. Sea $\epsilon$ suficientemente chico como para que la suma de los semi-anchos de ambas resonancias $\left(S_{a}^{\epsilon}\right.$ y $\left.S_{b}^{\epsilon}\right)$ sea mucho menor a la distancia entre las correspondientes acciones resonantes. Entonces, las órbitas con condiciones iniciales en la capa estocástica asociada a $\boldsymbol{I}_{a}^{r}$ se mantendrán indefinidamente allí, sin posibilidad de alcanzar el entorno de la otra resonancia. En este caso se dice que existe caos local, pero no difusión, ya que la región accesible por estas órbitas es de tamaño apenas igual a $2 S_{a}^{\epsilon}$. Ambas capas estocásticas se encuentran aisladas la una de la otra debido a los toros KAM aún persistentes que corresponden a acciones intermedias entre $\boldsymbol{I}_{a}^{r}$ y $\boldsymbol{I}_{b}^{r}$. Sin embargo, existe un valor de $\epsilon$, a partir del cual las capas estocásticas asociadas a dichas resonancias se tocan entre sí, permitiendo que órbitas con condiciones iniciales en entorno de $\boldsymbol{I}_{a}^{r}$ alcancen el entorno de $\boldsymbol{I}_{b}^{r}$, y viceversa. En otras palabras, a partir de este valor de $\epsilon$ ambas resonancias se solapan mutuamente, permitiendo que haya una inestabilidad caótica de tamaño igual a $S_{a}^{\epsilon}+\left|\boldsymbol{I}_{a}^{r}-\boldsymbol{I}_{b}^{r}\right|+S_{b}^{\epsilon}$. En este caso se habla de difusión o transporte caótico local ya que la variación de las acciones está limitada al entorno de estas dos resonancias.

El criterio de overlap, para determinar cuando empieza la difusión entre dos resonancias, consiste en encontrar el valor de $\epsilon$ en el cual sus separatrices se tocan mutuamente. El criterio también ha sido modificado para computar el valor en el cual aparece transporte entre los entornos de un número mayor de resonancias. Además, mediante simplificaciones adicionales, el criterio permite computar el valor de $\epsilon$ a partir del cual está topológicamente permitida la existencia de difusión global sobre la mayoría del espacio de acciones. El criterio de overlap utilizado en este capítulo corresponde con esta última versión.

En su trabajo pionero sobre el mapa estándar Chirikov (1979) muestra que la aplicación del criterio de overlap a resonancias primarias o enteras sobreestima el valor real del parámetro crítico, $K_{\mathrm{c}}$. Al considerar resonancias de orden mayor, el criterio mejora pero mantiene un error relativo de aproximadamente $\sim 0.35$. Sólo al considerar el espesor de las capas estocásticas asociados a las resonancias primarias, es que reduce este error relativo a $\sim 0.07$.

El criterio de overlap ha sido usado para distintos tipos de problemas físicos. En el campo 
astronómico ha sido aplicado al estudio de las inestabilidades en problemas gravitatorios de más de dos cuerpos. Por ejemplo, Wisdom (1980) lo utiliza para estimar el valor crítico del parámetro perturbativo que inicia el comportamiento estocástico macroscópico en el problema de tres cuerpos restringido circular. Lissauer (1995) extiende el método para determinar la estabilidad global de sistemas planetarios y obtiene restricciones a las masas de algunas de las lunas de Urano. Mudryk y Wu (2006) encuentran que el overlap es responsable de la eyección de planetas en sistemas binarios. Fuera del ámbito astronómico ha sido aplicado al problema Fermi-Pasta-Ulam (Flach and Ponno, 2008) y al estudio de la ionización del átomo de Hidrógeno (Sacha and Zakrzewski, 1997), entre otros.

En este capítulo realizaremos un análisis similar al realizado por Chirikov, pero usando un sistema Hamiltoniano casi-integrable 2DoF y uno 3DoF. Es decir, dos y tres oscilladores cuárticos desacoplados perturbados por un término cúbico, respectivamente. Parte del trabajo con la versión 2DoF corresponde a trabajo publicado en la tesis de licenciatura Mestre (2006). Esta información se ha agregado porque permite introducir el método en forma pausada y conocer la geometría resonante para un caso más sencillo, antes de pasar al caso más complejo de mayor dimensionalidad. La versión $3 \mathrm{DoF}$ de este modelo ha sido estudiada por Cincotta et al. (2003) y por Giordano y Cincotta (2004), donde los autores investigan numéricamente las propiedades dinámicas globales del modelo y estiman el valor crítico $\epsilon_{\mathrm{c}}$ a partir del cual el sistema es globalmente caótico, valor para el cual menos del $10 \%$ de la superficie de energía corresponde a toros KAM. Mediante el uso del criterio de overlap derivamos tal valor crítico para el parámetro perturbativo $\epsilon$ considerando resonancias primarias y de órdenes superiores, y la serie de Fourier de la perturbación truncada a $\mathcal{O}\left(1 / 23^{2}\right)$ en sus coeficientes. No se considera el espesor de las capas estocásticas. Luego comparamos, para cada caso, el valor crítico teórico con el obtenido numéricamente. La forma de aplicar el método teórico difiere entre el caso $2 \mathrm{DoF}$ y el $3 \mathrm{DoF}$, como se verá a lo largo del capítulo. Además, existe diferencia entre las formas de computar las respectivas contrapartidas numéricas. El conocer este valor crítico es una condición esencial para el posterior estudio numérico de la difusión realizado en el capítulo 4. Tal como fue explicado en 1.3.3, $\epsilon_{c}$ separa aproximadamente los regímenes de Chirikov y de Nekhoroshev, dominados respectivamente, por presencia y ausencia de overlap global.

Este capítulo está organizado de la siguiente manera. En 3.1 se define el oscilador cuártico de un grado de libertad. El sistema dinámico bajo estudio en su versión 2DoF es descripto en la sección 3.2, sus resonancias relevantes a $\mathcal{O}(\epsilon)$ son obtenidas en 3.3 y sus anchos calculados en 3.4. Posteriormente, las resonancias a $\mathcal{O}\left(\epsilon^{2}\right)$ son provistas en 3.5, donde se da una estimación del valor crítico del parámetro perturbativo. La comparación del resultado teórico con una estimación numérica se realiza en 3.6, mediante la realización de varias superficies de sección del sistema. El modelo 3 DoF es presentado en 3.7, cuyas resonancias a orden $\mathcal{O}(\epsilon)$ y $\mathcal{O}\left(\epsilon^{2}\right)$ son dadas en 3.8 y 3.9 , respectivamente. En 3.10 se analiza el cálculo teórico de la estimación del parámetro crítico, que resulta estar en muy buen acuerdo con el valor obtenido en Giordano y Cincotta (2004).

El material presentado en este capítulo ha sido publicado en Mestre et al. (2009). 


\subsection{El oscilador cuártico}

El oscilador cuártico de un grado de libertad esta dado por el Hamiltoniano

$$
\tilde{H}(p, q)=\frac{p^{2}}{2}+\frac{q^{4}}{4},
$$

y el sistema de ecuaciones diferenciales asociadas (1.3) es el siguiente:

$$
\frac{d p}{d t}=-q^{3}, \quad \frac{d q}{d t}=p .
$$

El hecho de que este sistema posee $n_{d}=1$ grados de libertad junto con la existencia de la integral de movimiento energía $(H(p, q)=h=$ cte $)$ son condicione suficientes para asegurar la integrabilidad del sistema (sección 1.2.2). De hecho, siendo a la amplitud de oscilación $\left(h=\frac{a^{4}}{4}\right)$, la Ec. 3.2 puede reescribirse:

$$
\left(\frac{d q}{d t}\right)^{2}=\frac{1}{2}\left(a^{4}-q^{4}\right)
$$

cuya solución se puede expresar en términos del coseno elíptico de Jacobi de módulo $k=\frac{1}{\sqrt{2}}$ :

$$
q(t)=a \operatorname{cn}(a t, k)=a \operatorname{cn}(a t, 1 / \sqrt{2}) .
$$

Utilizando el desarrollo en serie de Fourier del coseno elíptico dado por Gradshtyn y Ryzhik (1980), se tiene (Chirikov, 1979):

$$
q(t)=a \frac{\sqrt{2} \pi}{K_{0}} \sum_{n=1}^{\infty} \frac{1}{\cosh ((n-1 / 2) \pi)} \cos \left((2 n-1) \frac{\pi a t}{2 K_{0}}\right),
$$

donde $K_{0} \equiv K(1 / \sqrt{2})$ denota al valor de la integral elíptica completa de primera especie.

Introduciendo las constantes:

$$
\beta \equiv \frac{\pi}{2 K_{0}} \approx 0.847, \quad \alpha_{n} \equiv \frac{1}{\cosh ((n-1 / 2) \pi)} \quad \mathrm{y} \quad \omega \equiv \beta a
$$

se tiene que

$$
q(t)=2^{3 / 2} \omega \sum_{n=1}^{\infty} \alpha_{n} \cos ((2 n-1) \omega t)
$$

Los coeficientes $\alpha_{n}$ satisfacen:

$$
\frac{\alpha_{n+1}}{\alpha_{n}} \approx \frac{1}{23} \quad \text { para } \mathrm{n} \geq 2 \quad \mathrm{y} \quad \alpha_{1} \approx 0.4
$$

A partir la relación entre $a$ y $h$ mencionada anteriormente y de la Ec. (3.6) se obtiene la dependencia de la frecuencia $\omega$ con $h$ :

$$
\omega=\sqrt{2} \beta h^{1 / 4} .
$$

Dado que las ecuaciones de Hamilton en variables ángulo acción implican que $\omega(I)=\frac{\partial H(I)}{\partial I}$ es posible integrar (3.9) obteniendo la relación entre la acción y la energía del sistema:

$$
h=A I^{4 / 3}, \quad \text { o equivalentemente } \quad I=\left(\frac{h}{A}\right)^{3 / 4},
$$


donde $A \equiv(3 \beta / 2 \sqrt{2})^{4 / 3}$. A modo de completitud se menciona que la dependencia de la frecuencia con la acción es:

$$
\omega(I)=\frac{4}{3} A I^{1 / 3}
$$

La coordenada cartesiana $q$ puede expresarse en términos de las variables ángulo-acción $(\theta, I)$ del sistema de la siguiente forma:

$$
\begin{aligned}
& q(I, \theta)=(3 \beta I)^{1 / 3} \operatorname{cn}\left(\frac{\theta}{\beta}, \frac{1}{\sqrt{2}}\right) \\
& p(I, \theta)=\varrho \sqrt{2\left(A I^{4 / 3}-\frac{1}{4}[q(I, \theta)]^{4}\right)}
\end{aligned}
$$

donde $\varrho$ es el signo de $p$ y se determina en función del ángulo de la siguiente forma:

$$
\varrho \equiv\left\{\begin{aligned}
1 & \text { si } 0 \leq \theta<\pi \\
-1 & \text { si } \pi \leq \theta<2 \pi
\end{aligned}\right.
$$

A su vez, la tranformación inversa de (3.12) está dada por:

$$
\begin{aligned}
& I(p, q)=\left[\frac{1}{A}\left(\frac{1}{2} p^{2}+\frac{1}{4} q^{4}\right)\right]^{3 / 4}, \\
& \theta(p, q)= \begin{cases}\beta \mathrm{cn}^{-1}\left(\frac{q}{[3 \beta I(p, q)]^{1 / 3}}, \frac{1}{\sqrt{2}}\right) & \text { if } p \geq 0, \\
2 \pi-\beta \mathrm{cn}^{-1}\left(\frac{q}{[3 \beta I(p, q)]^{1 / 3}}, \frac{1}{\sqrt{2}}\right) & \text { if } p<0 .\end{cases}
\end{aligned}
$$

\subsection{El modelo dinámico $2 \mathrm{DoF}$}

A continuación analizaremos un sistema Hamiltoniano con dos grados de libertad que consiste en dos osciladores cuárticos perturbados. En coordenadas cartesianas, $\mathbf{q}=(x, y) \mathrm{y}$ $\mathbf{p}=\left(p_{x}, p_{y}\right)$, el sistema es descripto por el siguiente Hamiltoniano (Cincotta et al., 2003):

$$
\tilde{H}(\mathbf{p}, \mathbf{q})=\frac{1}{2}\left(p_{x}^{2}+p_{y}^{2}\right)+\frac{1}{4}\left(x^{4}+y^{4}\right)+\epsilon x^{2} y,
$$

donde $\epsilon$ es un parámetro perturbativo que controla la fuerza de la perturbación. Al fijar $\epsilon=0$ se obtiene el Hamiltoniano integrable de dos oscilador cuárticos libres. Utilizando (3.12) se puede expresar las coordenadas espaciales cartesianas en términos de las respectivas variables ángulo-acción:

$$
\begin{aligned}
& x\left(I_{1}, \theta_{1}\right)=\left(3 \beta I_{1}\right)^{1 / 3} \operatorname{cn}\left(\frac{\theta_{1}}{\beta}, \frac{1}{\sqrt{2}}\right) \\
& y\left(I_{2}, \theta_{2}\right)=\left(3 \beta I_{2}\right)^{1 / 3} \operatorname{cn}\left(\frac{\theta_{2}}{\beta}, \frac{1}{\sqrt{2}}\right) .
\end{aligned}
$$

Análogamente, utilizando algunos resultados y definiciones de la sección anterior, se define el vector de frecuencias, $\boldsymbol{\omega}$, como:

$$
\boldsymbol{\omega}(\boldsymbol{I}) \equiv\left(\omega_{1}\left(I_{1}\right), \omega_{2}\left(I_{2}\right)\right)=\frac{4}{3} A\left(I_{1}^{1 / 3}, I_{2}^{1 / 3}\right) .
$$


Utilizando Ecs. (3.17), (3.18) , (3.19) y el desarrollo de Fourier del coseno elíptico análogo al utilizado en (3.7), el Hamiltoniano completo se expresa en términos de las variables ánguloacción del Hamiltoniano no perturbado así:

$$
H(\boldsymbol{I}, \boldsymbol{\theta})=H_{0}(\boldsymbol{I})+\epsilon V(\boldsymbol{I}, \boldsymbol{\theta}),
$$

donde

$$
\begin{aligned}
H_{0}(\boldsymbol{I})= & A\left(I_{1}^{4 / 3}+I_{2}^{4 / 3}\right) \\
V(\boldsymbol{I}, \boldsymbol{\theta})= & 3 \beta I_{1}^{2 / 3} I_{2}^{1 / 3} \operatorname{cn}^{2}\left(\frac{\theta_{1}}{\beta}, \frac{1}{\sqrt{2}}\right) \operatorname{cn}\left(\frac{\theta_{2}}{\beta}, \frac{1}{\sqrt{2}}\right) \\
= & \hat{V}(\boldsymbol{I}) \sum_{n, m, k=1}^{\infty} \alpha_{n m k}\left\{\cos \left(2(n+m-1) \theta_{1} \pm(2 k-1) \theta_{2}\right)\right. \\
& \left.+\cos \left(2(n-m) \theta_{1} \pm(2 k-1) \theta_{2}\right)\right\}
\end{aligned}
$$

con $\alpha_{n m k} \equiv \alpha_{n} \alpha_{m} \alpha_{k}$, y $\hat{V}(\boldsymbol{I}) \equiv 2^{5 / 2} 3 \beta^{4} I_{1}^{2 / 3} I_{2}^{1 / 3}$, el signo \pm significando que ambos términos están incluídos en la serie.

\subsection{Resonancias a $\mathcal{O}(\epsilon)$}

Una mirada a la serie perturbativa dada en la ecuación (3.21) revela que el número de términos resonantes a primer orden en el parámetro perturbativo no es finito, lo cual hace imposible tener en cuenta numéricamente el ancho de cada resonancia a tal orden.

Sin embargo, la fuerte dependencia de las amplitudes de Fourier respecto de $(n+m+k)$, a través de las cantidades $\alpha_{n m k} \approx 1 / 23^{(n+m+k-3)}$, nos brinda ayuda respecto a cómo agrupar las resonancias de $\mathcal{O}(\epsilon)$ y dónde cortar la serie. Nuestra aproximación es quedarnos solamente con los términos hasta orden $\mathcal{O}\left(1 / 23^{2}\right)$.

Todas las posibles cominaciones de $n, m$ y $k$ que verifican que $n+m+k \leq 5$, nos dan 24 diferentes vectores listados en Tabla 3.1, junto con el número de veces que aparecen en un término con coeficiente $\alpha_{n m k}$ de un dado orden en $1 / 23$. Así, $N_{0}$ denota el número de veces que un vector aparece con coeficiente $\alpha_{n m k}=\alpha_{1}^{3}, N_{1}$ el número de veces que aparece con coeficiente $\alpha_{1}^{2} \alpha_{2}\left(\approx \alpha_{1}^{3} / 23\right)$, y $N_{2}$ corresponde al coeficiente $\alpha_{1} \alpha_{2}^{2}$ o $\alpha_{1}^{2} \alpha_{3}$ (que es aproximadamente $\left.\alpha_{1}^{3} / 23^{2}\right)$. Estos grupos de vectores serán respectivamente señalados como armónicos a $\mathcal{O}(\epsilon, 1), \mathcal{O}(\epsilon, 1 / 23)$ y $\mathcal{O}\left(\epsilon, 1 / 23^{2}\right)$. Juntos constituyen los vectores armónicos a $\mathcal{O}(\epsilon)$ hasta $\mathcal{O}\left(1 / 23^{2}\right)$.

Al aplicar la condición de resonancia $\boldsymbol{m} \cdot \boldsymbol{\omega}=0$, con $\boldsymbol{m} \in \mathbb{Z}^{2} \backslash\{\mathbf{0}\}$, al sistema no perturbado, la siguiente relación entre las energías en cada grado de libertad es obtenida:

$$
m_{1} h_{1}^{1 / 4}+m_{2} h_{2}^{1 / 4}=0,
$$

lo cual implica que la estructura resonante en ambos el espacio de acciones y el de energía consiste en líneas rectas (con pendiente positiva) dadas por

$$
h_{2}^{r}=\frac{m_{1}^{4}}{m_{2}^{4}} h_{1}^{r},
$$




\begin{tabular}{|c|c|c|c|c|c|c|c|}
\hline vector & $N_{0}$ & $N_{1}$ & $N_{2}$ & vector & $N_{0}$ & $N_{1}$ & $N_{2}$ \\
\hline$(2,1)$ & 1 & 1 & 0 & $(2,-1)$ & 1 & 1 & 0 \\
$(0,1)$ & 1 & 0 & 1 & $(0,-1)$ & 1 & 0 & 1 \\
$(2,3)$ & 0 & 1 & 1 & $(2,-3)$ & 0 & 1 & 1 \\
$(0,3)$ & 0 & 1 & 0 & $(0,-3)$ & 0 & 1 & 0 \\
$(2,5)$ & 0 & 0 & 1 & $(2,-5)$ & 0 & 0 & 1 \\
$(0,5)$ & 0 & 0 & 1 & $(0,-5)$ & 0 & 0 & 1 \\
$(4,1)$ & 0 & 2 & 1 & $(4,-1)$ & 0 & 2 & 1 \\
$(-2,1)$ & 0 & 1 & 0 & $(-2,-1)$ & 0 & 1 & 0 \\
$(4,3)$ & 0 & 0 & 2 & $(4,-3)$ & 0 & 0 & 2 \\
$(-2,3)$ & 0 & 0 & 1 & $(-2,-3)$ & 0 & 0 & 1 \\
$(6,1)$ & 0 & 0 & 3 & $(6,-1)$ & 0 & 0 & 3 \\
$(-4,1)$ & 0 & 0 & 1 & $(-4,-1)$ & 0 & 0 & 1 \\
\hline
\end{tabular}

Tabla 3.1: Armónicos en la expansión de Fourier (3.21) a $\mathcal{O}(\epsilon)$ hasta $\mathcal{O}\left(1 / 23^{2}\right)$

y

$$
I_{2}^{r}=\left|\frac{m_{1}^{3}}{m_{2}^{3}}\right| I_{1}^{r},
$$

respectivamente.

Además, Ec. (3.22) indica que $m_{1} m_{2} \leq 0$, por lo que, aquellos vectores que poseen ambas componentes con el mismo signo no pueden ser resonantes. Observemos, sin embargo, que no todos los restantes armónicos a $\mathcal{O}(\epsilon)$ hasta $\mathcal{O}\left(1 / 23^{2}\right)$ son resonantes, como será discutido en la sección siguiente.

\subsection{Ancho de las resonancias a $\mathcal{O}(\epsilon)$}

Al computar los anchos de resonancias, la aproximación del péndulo (ver por ejemplo sección 1.4.4, Chirikov 1979 o Cincotta 2002) nos provee de una descripción adecuada siempre que cada resonancia se asuma suficientemente aislada del resto.

Antes de proceder a estimar el ancho de una dada resonancia, todos los coeficientes $\alpha_{n m k}$ asociados a la misma función trigonométrica se adicionarán en un sólo coeficiente. De hecho, para cada vector $\boldsymbol{m}$, definimos el coeficiente:

$$
\alpha_{m} \equiv \sum_{m+n+k \leq 5} \alpha_{n m k},
$$

donde $n, m, \mathrm{y} k$ son números naturales que se combinan para formar el vector $\boldsymbol{m}$ en cualquiera de las cuatro formas desplegadas en Ec. (3.21). Debe notarse que

$$
\alpha_{\boldsymbol{m}}=\alpha_{1}^{3}\left(N_{0}+N_{1} / 23+N_{2} / 23^{2}\right)+\mathcal{O}\left(1 / 23^{3}\right) .
$$

Una vez que la aproximación del péndulo ha sido aplicada a este término resonante aislado, el nuevo Hamiltonian (resonante) resulta ser:

$$
H_{r}\left(p_{1}, \psi_{1}\right)=\frac{p_{1}^{2}}{2 M}+\epsilon \hat{V}\left(\boldsymbol{I}^{\boldsymbol{r}}\right) \alpha_{m} \cos \psi_{1},
$$


con

$$
M^{-1} \equiv \sum_{i} \sum_{j} \frac{\partial \omega_{i}^{r}}{\partial I_{j}} m_{j}=\sum_{i} m_{i}^{2} \frac{\partial \omega_{i}^{r}}{\partial I_{i}}, \quad \boldsymbol{I}=\boldsymbol{I}^{\boldsymbol{r}}+\boldsymbol{m} p_{1}, \quad \psi_{1}=\boldsymbol{m} \cdot \boldsymbol{\theta},
$$

donde se ha utilizado el hecho de que $\omega_{i}^{r}$ no depende de $I_{j}^{r}$ para $j \neq i$.

La expresión de la solución para el momento en la separatriz del péndulo descripto por Ec. (3.26) está dada por:

$$
p_{s}\left(\psi_{s}\right)= \pm 2 \sqrt{M \epsilon \hat{V}\left(\boldsymbol{I}^{\boldsymbol{r}}\right) \alpha_{\boldsymbol{m}}} \sin \left(\psi_{s} / 2\right)
$$

La diferencia de esta ecuación respecto de (1.34) se debe a la diferencia, en un signo, entre $(1.27)$ y (3.26).

Sea $p_{r}$ la máxima variación de $p_{1}$ dentro del régimen de oscilación. Dado que dicha cantidad coincide con el máximo valor alcanzado en la separatriz, se tiene que:

$$
p_{r}=2\left(\epsilon M \hat{V}\left(\boldsymbol{I}^{r}\right) \alpha_{n m k}\right)^{1 / 2}=2^{7 / 2} \beta^{1 / 2}\left|\frac{m_{1}^{3} m_{2}^{-1}}{m_{1}^{4}+m_{2}^{4}}\right|^{1 / 2} \epsilon^{1 / 2} \alpha_{n m k}^{1 / 2}\left(h_{1}^{r}\right)^{5 / 8}
$$

Como consecuencia de la dinámica del péndulo simple, el máximo desplazamiento de las acciones no perturbadas depende de $\boldsymbol{m}$ y $p_{r}$ de la forma: $(\Delta \boldsymbol{I})^{\boldsymbol{r}} \equiv\left(\boldsymbol{I}-\boldsymbol{I}^{\boldsymbol{r}}\right)_{\max }=p_{r} \boldsymbol{m}$.

Además, el máximo desplazamiento de la energía no perturbada está dado por $\left|\left(\Delta h_{i}\right)_{m}^{r}\right|=$ $\left|\omega_{i}^{r}\left(\Delta I_{i}\right)_{m}^{r}\right|$; y es la máxima amplitud alcanzada en la oscilación de cualquiera de las energías no perturbadas que mide el ancho de la resonancia.

Recordemos ahora que en cualquier problema de 2DoF la condición de conservación de la energía

$$
h=h_{1}+h_{2}
$$

junto con la condición resonante (3.22) permiten a ambas $h_{1}^{r}$ y $h_{2}^{r}$ ser escritas en términos de la energía total no perturbada $h$.

Así, la amplitud puede ser reescrita en términos de $m_{1}, m_{2}, \epsilon, \alpha_{\boldsymbol{m}}$ y $h$ como sigue:

$$
\begin{aligned}
\left|\left(\Delta h_{1}\right)_{m}^{r}\right| & =2^{4} \beta^{3 / 2} \frac{\left|m_{1}\right|^{5 / 2}\left|m_{2}\right|^{3}}{\left|m_{1}^{4}+m_{2}^{4}\right|^{11 / 8}} \epsilon^{1 / 2} \alpha_{\boldsymbol{m}}^{1 / 2} h^{r 7 / 8} \\
\left|\left(\Delta h_{2}\right)_{\boldsymbol{m}}^{r}\right| & =\left|\left(\Delta h_{1}\right)_{\boldsymbol{m}}^{r}\right| .
\end{aligned}
$$

La última identidad en (3.31) es debida al hecho de que en presencia de una sola resonancia, el movimiento del sistema es tangente a la superficie de energía no perturbada, y para este modelo particular tal superficie esta dada por (3.30), lo cual nos lleva a $\Delta h_{2}=-\Delta h_{1}$.

Podemos notar que el ancho de una resonancia a $\mathcal{O}(\epsilon)$ hasta $\mathcal{O}\left(1 / 23^{2}\right)$ depende de los números armónicos en la manera mostrada en la Fig. 3.1, por lo que aquellas resonancias con valores de $\gamma=m_{2} / m_{1}$ fuera del rango [0.5, 2.5] son angostas.

Una mirada a la Ec. (3.31) y a la Fig. 3.1 revela que aquellos vectores armónicos con alguna de sus components igual a cero no manifiestan variaciones en las energías, y consecuentemente, no deben ser considerados como vectores resonantes.

Asimismo, debido a que los términos perturbativos son pares, si $\boldsymbol{m}$ es un vector resonante entonces $-\boldsymbol{m}$ es igualmente un vector resonante (ambos correspondientes a la misma resonancia). Por lo tanto, para cada resonancia consideramos un sólo vector resonante representativo, 


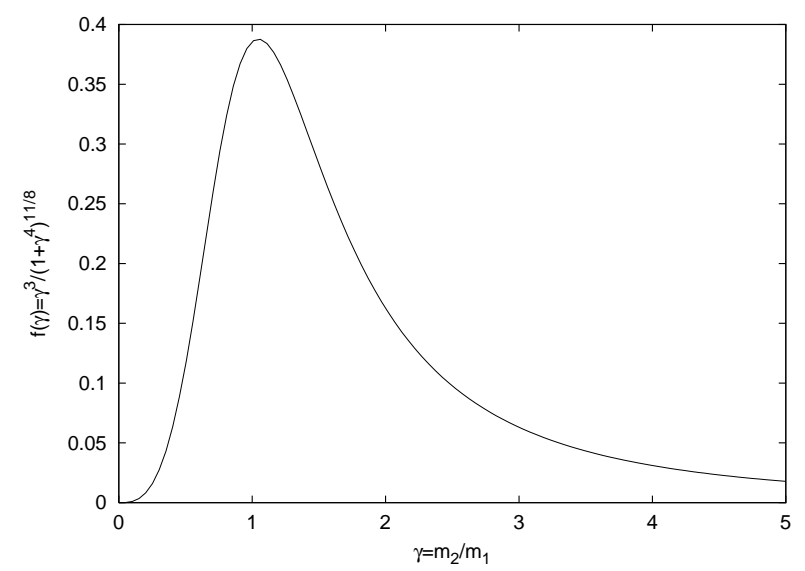

Figura 3.1: Dependencia del ancho de la resonancia respecto a $\gamma \equiv m_{2} / m_{1}$.

pero se incluye en su coeficiente asociado la contribución correspondiente a su vector opuesto. Toda la información relevante requerida para computar el ancho de cada resonancia a $\mathcal{O}(\epsilon)$ hasta $\mathcal{O}\left(1 / 23^{2}\right)$ está expuesto en la Tabla 3.2, donde hemos además incluído el valor de $h_{1}^{r}$ correspondiente a una energía no perturbada total de $h=0.485$ (similar a la utilizada en Cincotta et al. 2003). Este valor de $h$ corresponde a un período característico ${ }^{1}$ del sistema de valor $\approx 2 \pi$ y será utilizado durante toda la tesis.

\begin{tabular}{|c|c|c|c|c|}
\hline vector & $N_{0}$ & $N_{1}$ & $N_{2}$ & $h_{1}^{r}$ \\
\hline$(2,-5)$ & 0 & 0 & 1 & 0.4729 \\
$(2,-3)$ & 0 & 1 & 2 & 0.4050 \\
$(4,-3)$ & 0 & 0 & 2 & 0.1166 \\
$(2,-1)$ & 1 & 2 & 0 & 0.0285 \\
$(4,-1)$ & 0 & 2 & 2 & 0.0019 \\
$(6,-1)$ & 0 & 0 & 3 & 0.0004 \\
\hline
\end{tabular}

Tabla 3.2: Vectores resonantes a $\mathcal{O}(\epsilon)$ hasta $\mathcal{O}\left(1 / 23^{2}\right)$.

Hemos computado los anchos de resonancia correspondientes a $\epsilon$ en el rango $[0,0.5]$. La Fig. 3.2 muestra los valores máximo y mínimo de $h_{1}$ para cada resonancia contra el parámetro perturbativo $\epsilon$; siendo la energía total no perturbada $h=1 /\left(4 \beta^{4}\right)$. Lo que se observa es que para $\epsilon \sim 0.15$, las resonancias $(6,-1),(4,-1),(2-1)$ y $(4,-3)$ se solapan entre sí pero se mantienen separadas del par de resonancias $(2,-3)$ y $(2,-5)$. Por lo tanto, podríamos inferir que la superficie de energía presenta dos regiones disconexas de movimiento caótico, por lo que una transición global al caos no toma lugar para $\epsilon \lesssim 0.5$, lo cual lleva a un valor teórico del parámetro perturbativo crítico $\epsilon_{\mathrm{c}} \gg 0.5$.

\subsection{Resonancias a $\mathcal{O}\left(\epsilon^{2}\right)$}

En regiones del espacio de fases que están suficientemente lejos de cualquier resonancia primaria (por ejemplo: condición diofántica), es posible introducir nuevas variables canónicas

\footnotetext{
${ }^{1}$ En este caso se refiere al período de las órbitas periódicas de eje $y$ o $z$, que permanecen estables para todo valor de $\epsilon$.
} 


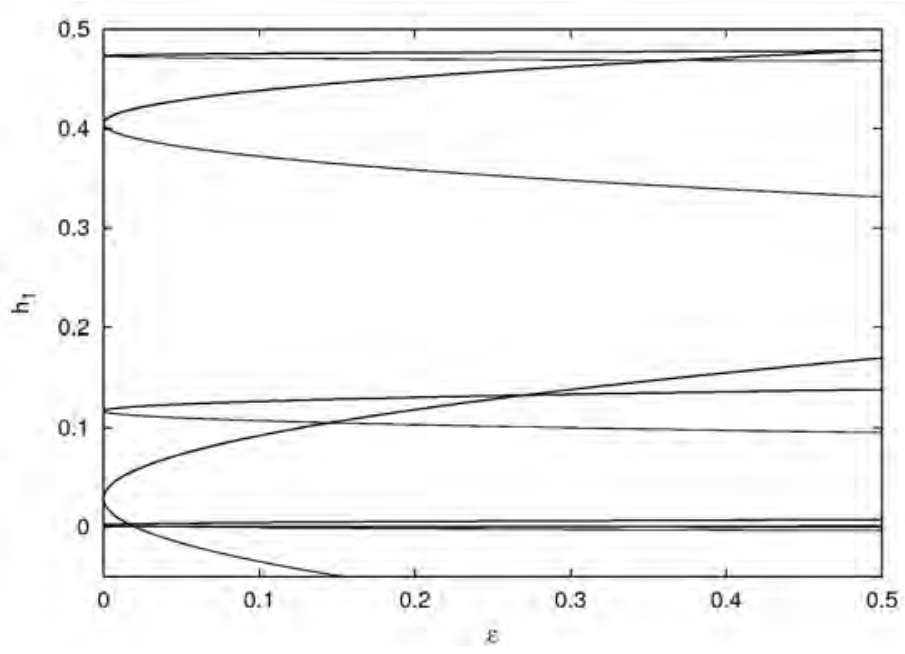

Figura 3.2: Anchos de las resonancias a $\mathcal{O}(\epsilon)$ hasta $\mathcal{O}\left(1 / 23^{2}\right)$, en términos de $\epsilon$.

$(\boldsymbol{J}, \boldsymbol{\varphi})$, de forma tal que el Hamiltoniano transformado consiste de una parte que depende del nuevo momento y una perturbación que, aunque no integrable, posee una amplitud de $\mathcal{O}\left(\epsilon^{2}\right)$.

$$
\begin{aligned}
\mathcal{H}(\boldsymbol{J}, \boldsymbol{\varphi}) & =H_{0}(\boldsymbol{J})+\epsilon^{2}\left\{\frac{1}{2} \frac{\partial^{2} H_{0}(\boldsymbol{J})}{\partial J_{j} \partial J_{i}} \frac{\partial \Phi(\boldsymbol{J}, \boldsymbol{\varphi})}{\partial \varphi_{j}} \frac{\partial \Phi(\boldsymbol{J}, \boldsymbol{\varphi})}{\partial \varphi_{i}}+\frac{\partial V(\boldsymbol{J}, \boldsymbol{\varphi})}{\partial J_{i}} \frac{\partial \Phi(\boldsymbol{J}, \boldsymbol{\varphi})}{\partial \varphi_{i}}\right\} \\
& +\mathcal{O}\left(\epsilon^{3}\right),
\end{aligned}
$$

donde $\Phi$ representa la parte trigonométrica de la función generatriz de la transformación canónica:

$$
F(\boldsymbol{J}, \boldsymbol{\theta})=\boldsymbol{J} \cdot \boldsymbol{\theta}+\epsilon \Phi(\boldsymbol{J}, \boldsymbol{\theta}) \quad \Phi(\boldsymbol{J}, \boldsymbol{\theta})=\sum_{\boldsymbol{m} \in \mathbb{Z}^{2} \backslash\{\mathbf{0}\}} \Phi_{\boldsymbol{m}}(\boldsymbol{J}) \sin (\boldsymbol{m} \cdot \boldsymbol{\theta}),
$$

$\operatorname{con} \Phi_{\boldsymbol{m}}(\boldsymbol{J})=-V_{\boldsymbol{m}}(\boldsymbol{J}) / \boldsymbol{m} \cdot \boldsymbol{\omega}(\boldsymbol{J})$.

Después de computar el lado derecho de la expresión (3.32) se encuentra:

$$
\begin{aligned}
\mathcal{H}(\boldsymbol{J}, \boldsymbol{\varphi})=H_{0}(\boldsymbol{J})+\epsilon^{2} \sum_{\boldsymbol{m}, \boldsymbol{m}^{\prime}} \mathbb{C}\left(\boldsymbol{m}, \boldsymbol{m}^{\prime}, \boldsymbol{J}\right)\left\{\cos \left(\left(\boldsymbol{m}+\boldsymbol{m}^{\prime}\right) \cdot \boldsymbol{\varphi}\right)\right. & \\
& \left.+\cos \left(\left(\boldsymbol{m}-\boldsymbol{m}^{\prime}\right) \cdot \boldsymbol{\varphi}\right)\right\}+\mathcal{O}\left(\epsilon^{3}\right),
\end{aligned}
$$

donde los coeficientes están dados por

$$
\begin{aligned}
\mathbb{C}\left(\boldsymbol{m}, \boldsymbol{m}^{\prime}, \boldsymbol{J}\right)=\alpha_{\boldsymbol{m}} \alpha_{\boldsymbol{m}^{\prime}} & \left\{2^{3} 3^{4 / 3} \beta^{28 / 3} \frac{\left(m_{1} m_{1}^{\prime} J_{1}^{2 / 3} J_{2}^{2 / 3}+m_{2} m_{2}^{\prime} J_{1}^{4 / 3}\right)}{(\boldsymbol{m} \cdot \boldsymbol{\omega}(\boldsymbol{J}))\left(\boldsymbol{m}^{\prime} \cdot \boldsymbol{\omega}(\boldsymbol{J})\right)}\right. \\
& \left.-2^{4} 3 \beta^{8} \frac{\left(2 m_{1}^{\prime} J_{1}^{1 / 3} J_{2}^{2 / 3}+m_{2}^{\prime} J_{1}^{4 / 3} J_{2}^{-1 / 3}\right)}{\left(\boldsymbol{m}^{\prime} \cdot \boldsymbol{\omega}(\boldsymbol{J})\right)}\right\} .
\end{aligned}
$$

Deben hacerse las siguientes aclaraciones:

(i) Al trabajar a $\mathcal{O}(\epsilon)$ se han considerado sólo las resonancias hasta $\mathcal{O}\left(1 / 23^{2}\right)$ en los coeficientes de Fourier. Por lo tanto, la serie en Ec. (3.33) es reemplazada por una suma finita 
sobre aquellos armónicos, $\boldsymbol{m}$ y $\boldsymbol{m}^{\prime}$, cuyos coeficientes asociados $\left(\alpha_{\boldsymbol{m}}\right.$ y $\alpha_{\boldsymbol{m}^{\prime}}$, respectivamente) son tales que su producto es de $\mathcal{O}(1), \mathcal{O}(1 / 23)$ o $\mathcal{O}\left(1 / 23^{2}\right)$; (ii) existen muchos pares de armónicos distintos $\left(\boldsymbol{m}, \boldsymbol{m}^{\prime}\right)$ a $\mathcal{O}(\epsilon)$, que se combinan formando el mismo armónico, $\boldsymbol{n}$, a $\mathcal{O}\left(\epsilon^{2}\right)$; (iii) como consecuencia de la paridad de los términos perturbativos, si $\boldsymbol{n}$ es un vector resonante, entonces $\boldsymbol{-} \boldsymbol{n}$ también lo es; (iv) la condición de resonancia implica que $n_{1} n_{2} \leq 0$; y $(\mathrm{v})$ la condición de estar lejos de las resonancias a $\mathcal{O}(\epsilon)$ implica que debemos descartar todo aquel armónico que es un múltiplo de cualquier vector resonante a $\mathcal{O}(\epsilon)$ hasta $\mathcal{O}\left(1 / 23^{2}\right)$.

Para resolver la situación planteada por los incisos (ii) y (iii), se han sumado todas las correspondientes contribuciones en un sólo coeficiente, es decir,

$$
\mathbb{D}(\boldsymbol{n}, \boldsymbol{J})=\sum_{\boldsymbol{m}, \boldsymbol{m}^{\prime}} \mathbb{C}\left(\boldsymbol{m}, \boldsymbol{m}^{\prime}, \boldsymbol{J}\right),
$$

donde la suma se extiende hacia todos los armónicos $\left(\boldsymbol{m}, \boldsymbol{m}^{\prime}\right)$ a $\mathcal{O}(\epsilon)$ hasta $\mathcal{O}\left(1 / 23^{2}\right)$ tales que $\boldsymbol{n}=\boldsymbol{m}+\boldsymbol{m}^{\prime}, \boldsymbol{n}=\boldsymbol{m}-\boldsymbol{m}^{\prime},-\boldsymbol{n}=\boldsymbol{m}+\boldsymbol{m}^{\prime}$, o $-\boldsymbol{n}=\boldsymbol{m}-\boldsymbol{m}^{\prime}$, y para los cuales $\alpha_{\boldsymbol{m}} \alpha_{\boldsymbol{m}^{\prime}} \lesssim \alpha_{1}^{6} / 23^{2}$.

Teniendo en cuenta todas estas consideraciones, el Hamiltoniano puede ser escrito en la forma:

$$
\mathcal{H}(\boldsymbol{J}, \boldsymbol{\varphi})=H_{0}(\boldsymbol{J})+\epsilon^{2} \sum_{\boldsymbol{n}} \mathbb{D}(\boldsymbol{n}, \boldsymbol{J}) \cos (\boldsymbol{n} \cdot \boldsymbol{\varphi}) .
$$

Por lo tanto, en la vecindad de un toro resonante $\boldsymbol{J}^{r}$, y haciendo uso de la aproximación del péndulo, obtenemos el nuevo Hamiltoniano resonante:

$$
\mathcal{H}_{r}\left(\mathcal{P}_{1}, \Psi_{1}\right)=\frac{\mathcal{P}_{1}^{2}}{2 \mu}+\mathcal{U}_{0} \cos \left(\Psi_{1}\right)
$$

donde

$$
\begin{aligned}
\mu^{-1} & \equiv n_{i} \frac{\partial \omega_{i}^{r}}{\partial J_{j}} n_{j} \\
\mathcal{U}_{0} & \equiv \epsilon^{2} \mathbb{D}\left(\boldsymbol{n}, \boldsymbol{J}^{\boldsymbol{r}}\right)
\end{aligned}
$$

Así, el máximo desplazamiento de las acciones no perturbadas esta dada por $(\Delta \boldsymbol{J})^{r} \equiv$ $\left(\boldsymbol{J}-\boldsymbol{J}^{r}\right)_{\max }=\mathcal{P}_{r} \boldsymbol{n}$, con $\mathcal{P}_{r}=2\left(\mu\left|\mathcal{U}_{0}\right|\right)^{1 / 2}$, y el ancho de la resonancias a $\mathcal{O}\left(\epsilon^{2}\right)$ está dado por

$$
\begin{aligned}
\left|\left(\Delta h_{1}\right)_{\boldsymbol{n}}^{r}\right|= & 2^{4} \beta^{3} h^{3 / 4} \epsilon \frac{\left|n_{2}\right|^{3} n_{1}^{2}}{\left(n_{1}^{4}+n_{2}^{4}\right)^{5 / 4}} \\
& \times\left|\sum_{\boldsymbol{m}, \boldsymbol{m}^{\prime}} \alpha_{\boldsymbol{m}} \alpha_{\boldsymbol{m}^{\prime}}\left\{\frac{2\left(2\left|n_{1}\right|^{3} m_{1}^{\prime}+\left|n_{2}\right|^{3} m_{2}^{\prime}\right)}{\left|n_{1} n_{2}\right|\left(m_{1}^{\prime}\left|n_{2}\right|+m_{2}^{\prime}\left|n_{1}\right|\right)} \pm \frac{n_{1}^{2} m_{1}^{\prime} m_{1}+n_{2}^{2} m_{2}^{\prime} m_{2}}{\left(m_{1}^{\prime}\left|n_{2}\right|+m_{2}^{\prime}\left|n_{1}\right|\right)^{2}}\right\}\right|^{1 / 2},
\end{aligned}
$$

donde el signo "+" corresponde a $\boldsymbol{n}=\boldsymbol{m}+\boldsymbol{m}^{\prime}$ y el signo "-" corresponde a $\boldsymbol{n}=\boldsymbol{m}-\boldsymbol{m}^{\prime}$; $m_{i}$ debe ser escrito en términos de $m_{i}^{\prime}$ y $n_{i}$.

Los armónicos que satisfacen todas las condiciones establecidas para la perturbación a $\mathcal{O}\left(\epsilon^{2}\right)$ resultan ser sólo cinco, que están listados en la Tabla 3.3. Ellos serán llamados vectores resonantes a $\mathcal{O}\left(\epsilon^{2}\right)$ hasta $\mathcal{O}\left(1 / 23^{2}\right)$. Hemos computado los correspondientes anchos de 


\begin{tabular}{|c|c|}
\hline vector & $h_{1}^{r}$ \\
\hline$(2,-6)$ & 0.4791 \\
$(2,-4)$ & 0.4565 \\
$(2,-2)$ & 0.2425 \\
$(6,-4)$ & 0.0800 \\
$(6,-2)$ & 0.0059 \\
\hline
\end{tabular}

Tabla 3.3: Vectores resonantes a $\mathcal{O}\left(\epsilon^{2}\right)$ hasta $\mathcal{O}\left(1 / 23^{2}\right)$.

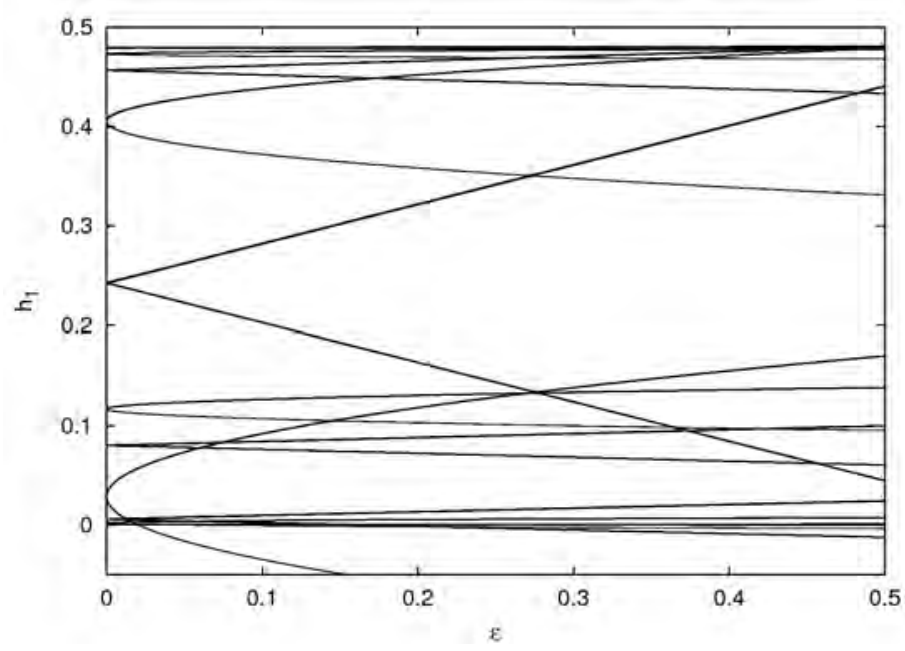

Figura 3.3: Ancho de las resonancias a $\mathcal{O}\left(\epsilon^{2}\right)$ hasta $\mathcal{O}\left(1 / 23^{2}\right)$, en términos de $\epsilon$.

resonancias para $\epsilon$ en el rango $[0,0.5]$. Los resultados están presentados en la Fig. 3.3, donde también se han incluído las resonancias $\mathcal{O}(\epsilon)$ hasta $\mathcal{O}\left(1 / 23^{2}\right)$ previamente graficadas.

De la Fig. 3.3 y la Tabla 3.3 se tiene que la nueva resonancia $(2,-2)$ conecta a dos conjuntos de resonancias que hasta $\mathcal{O}\left(\epsilon, 1 / 23^{2}\right)$ aparecían aisladas para el rango de $\epsilon$ considerado. Las restantes resonancias a $\mathcal{O}\left(\epsilon^{2}\right)$ hasta $\mathcal{O}\left(1 / 23^{2}\right)$ aparecen completamente solapadas con alguno de los dos conjuntos de las resonancias hasta $\mathcal{O}\left(\epsilon, 1 / 23^{2}\right)$. A partir de este gráfico, se ha podido derivar el valor crítico del parámetro perturbativo, $\epsilon_{\mathrm{c}} \approx 0.28$.

\subsection{Estimación numérica del valor crítico del parámetro per- turbativo}

En esta sección se estima, empírica y numéricamente, el valor de $\epsilon_{\mathrm{c}}$ mediante superficies de sección de Poincaré (SSP). Para ello, se tomaron las intersecciones sobre el plano $x=0$ (de hecho $|x|<10^{-8}$ ) siempre que $p_{x}>0$, para muchas condiciones iniciales a lo largo del eje $y$.

La Fig. 3.4 muestra las SSP correspondientes a $\epsilon=0.12$ (a la izquierda) y a $\epsilon=0.14$ (a la derecha), respectivamente. Allí se puede distinguir la resonancia $(2,-1)$, muy cerana a la última curva invariante que corresponde a la órbita periódica de eje $y$, la resonancia $(1,0)$. Además se pueden ver las resonancias $(2,-2)$ y $(2,-3)$. Las resonancias $(2,-4),(2,-5)$ y $(2,-6)$ no son visibles debido al hecho de que están completamente destruídas por overlap, 
como puede corroborarse en la Fig. 3.3, para este valor de $\epsilon$. Aparecen muchas otras resonancias de orden superior que no han sido teóricamente computadas.

Para $\epsilon=0.12$, se observa que el dominio caótico donde las resonancias $(2,-4),(2,-5)$ y $(2,-6)$ aparecen destruídas por overlap esta acotado por algunos toros KAM y así, permanece desconectado de la componente caótica externa alrededor de las resonancias $(2,-3)$ y $(2,-1)$. Por otro lado, para $\epsilon=0.14$, ambas zonas caóticas están conectadas, habiendo caos global, en el sentido de que la mayoría de las órbitas irregulares podrían explorar casi toda la componente caótica del espacio de fases.
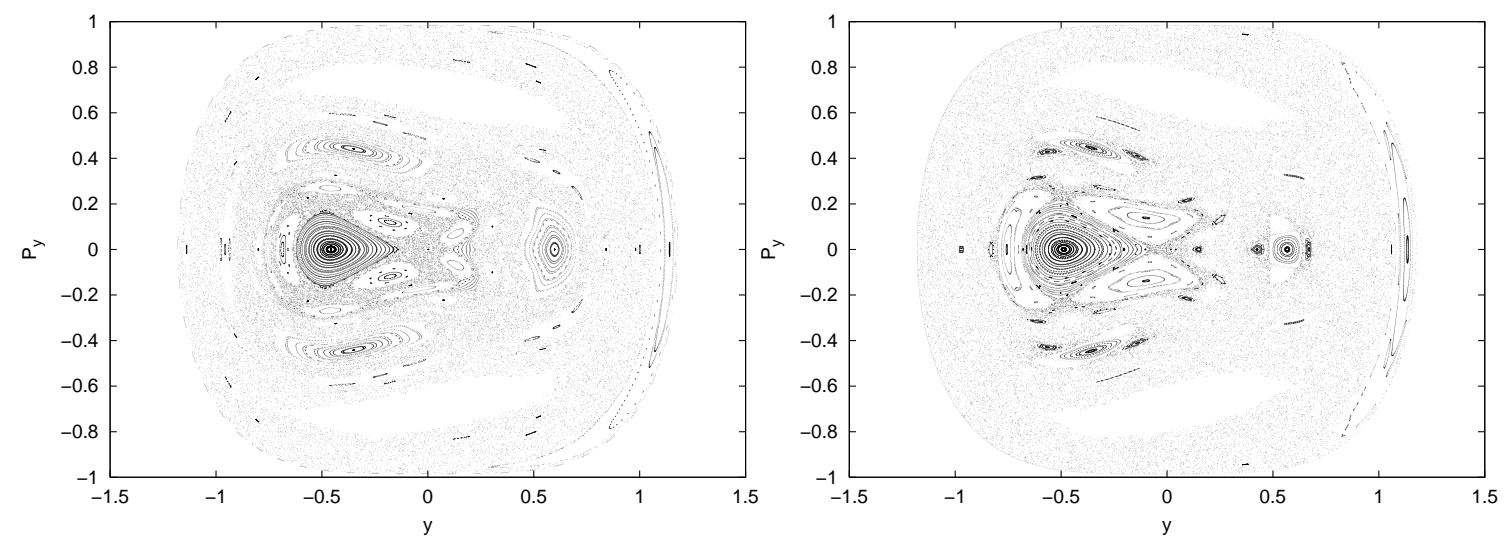

Figura 3.4: Superficies de sección de Poincaré para $\epsilon=0.12$ (a la izquierda), y para $\epsilon=0.14$ (a la derecha).

Por lo tanto, la evidencia determina que $\epsilon_{\mathrm{c}}$ yace en algún punto en el rango $(0.12,0.14)$. Luego de realizar una exploración numérica, se encontró que para $\epsilon=0.135$, varios toros KAM aún persisten, los cuales se muestran en la Fig. 3.5,donde se presenta una ampliación de la ventana $[0,0.4] \times[0,0.3]$.

Allí, tales toros KAM pueden distinguirse claramente y se observa cómo separan a ambos dominios caóticos en el espacio de fases. Sin embargo, esta región acotada de movimiento irregular no incluye a las resonancias que en este trabajo se está considerando para derivar la estimación analítica de $\epsilon_{\mathrm{c}}$, sino otras de orden superior.

Por lo tanto, a partir de medios experimentales, se puede decir que $\epsilon_{c} \gtrsim 0.135$ es una cota inferior para el valor de $\epsilon_{c}$.

\subsection{El modelo dinámico $3 \mathrm{DoF}$}

Ahora se focalizará en la versión 3DoF del sistema dinámico bajo estudio. En coordenadas cartesianas, $\mathbf{q}=(x, y, z)$ y $\mathbf{p}=\left(p_{x}, p_{y}, p_{z}\right)$, su Hamiltoniano está dado por:

$$
\tilde{H}(\mathbf{p}, \mathbf{q})=\frac{1}{2}\left(p_{x}^{2}+p_{y}^{2}+p_{z}^{2}\right)+\frac{1}{4}\left(x^{4}+y^{4}+z^{4}\right)+\epsilon x^{2}(y+z) .
$$

Para un valor nulo del parámetro perturbativo se obtienen tres osciladores cuárticos desacoplados, donde la forma funcional de $x\left(I_{1}, \theta_{1}\right)$ e $y\left(I_{2}, \theta_{2}\right)$ es equivalente a la dada en (3.18) para el sistema de $2 \mathrm{DoF}$, mientras que la tercera coordenada, $z$, adopta una forma análoga: 


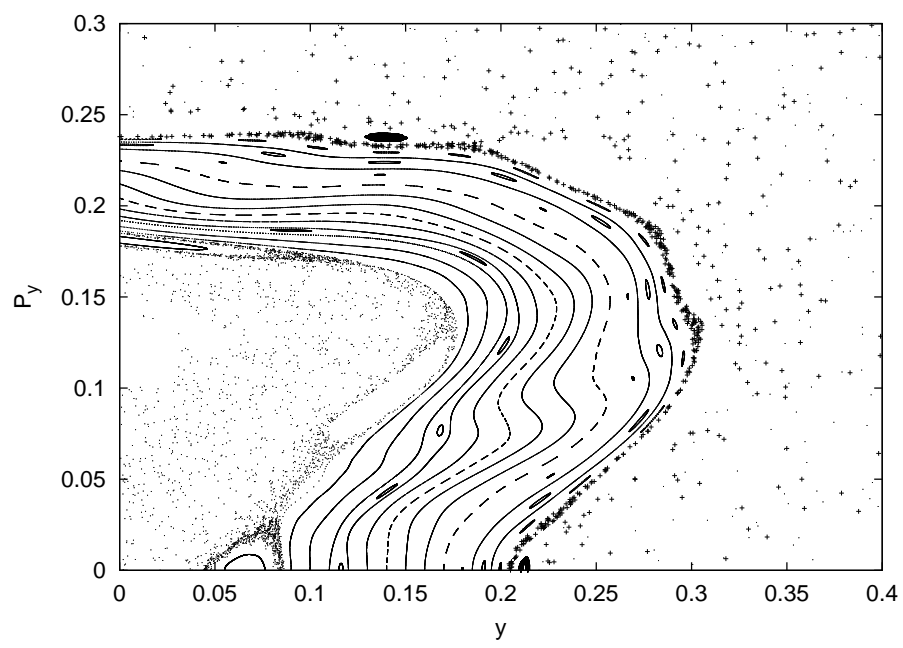

Figura 3.5: Superficie de sección de Poincaré para $\epsilon=0.135$, ilustrando la existencia de toros KAM que separan ambas componentes.

$$
z\left(I_{3}, \theta_{3}\right)=\left(3 \beta I_{3}\right)^{1 / 3} \mathrm{cn}\left(\frac{\theta_{3}}{\beta}, \frac{1}{\sqrt{2}}\right),
$$

y el vector de frecuencias queda:

$$
\boldsymbol{\omega}(\boldsymbol{I}) \equiv\left(\omega_{1}\left(I_{1}\right), \omega_{2}\left(I_{2}\right), \omega_{3}\left(I_{3}\right)\right)=\frac{4}{3} A\left(I_{1}^{1 / 3}, I_{2}^{1 / 3}, I_{3}^{1 / 3}\right) .
$$

En términos de las variables ángulo-acción del Hamiltoniano no perturbado, el Hamiltoniano completo (3.39) se reescribe así:

$$
H(\boldsymbol{I}, \boldsymbol{\theta})=H_{0}(\boldsymbol{I})+\epsilon V(\boldsymbol{I}, \boldsymbol{\theta}),
$$

con

$$
\begin{array}{rlrl}
H_{0}(\boldsymbol{I})=A\left(I_{1}^{4 / 3}+I_{2}^{4 / 3}+I_{3}^{4 / 3}\right), & \\
V(\boldsymbol{I}, \boldsymbol{\theta})=3 \beta I_{1}^{2 / 3} \operatorname{cn}^{2}\left(\frac{\theta_{1}}{\beta}, \frac{1}{\sqrt{2}}\right) & {\left[I_{2}^{1 / 3} \operatorname{cn}\left(\frac{\theta_{2}}{\beta}, \frac{1}{\sqrt{2}}\right)+\mathrm{I}_{3}^{1 / 3} \operatorname{cn}\left(\frac{\theta_{3}}{\beta}, \frac{1}{\sqrt{2}}\right)\right]} \\
=\hat{V}_{12}(\boldsymbol{I}) \sum_{n, m, k=1}^{\infty} \alpha_{n m k} & \left\{\cos \left(2(n+m-1) \theta_{1} \pm(2 k-1) \theta_{2}\right)\right. \\
& \left.+\cos \left(2(n-m) \theta_{1} \pm(2 k-1) \theta_{2}\right)\right\} \\
+\hat{V}_{13}(\boldsymbol{I}) \sum_{n, m, k=1}^{\infty} \alpha_{n m k} & \left\{\cos \left(2(n+m-1) \theta_{1} \pm(2 k-1) \theta_{3}\right)\right. \\
& & \left.+\cos \left(2(n-m) \theta_{1} \pm(2 k-1) \theta_{3}\right)\right\},
\end{array}
$$

donde se han introducido las nuevas cantidades $\hat{V}_{1 j}(\boldsymbol{I}) \equiv 2^{5 / 2} 3 \beta^{4} I_{1}^{2 / 3} I_{j}^{1 / 3}$; el signo \pm significando que ambos términos están incluídos en la serie. 


\subsection{Resonancias a $\mathcal{O}(\epsilon)$}

La perturbación dada en Ec. (3.43) muestra que para cada combinación de $n, m$ y $k$ existen ocho vectores armónicos, $\boldsymbol{m}$. Nuevamente, debido al carácter par de los términos perturbativos, se toma sólo un vector resonante representativo, $\boldsymbol{m}$, cuyo coeficiente $\alpha_{\boldsymbol{m}}$ también contiene la contribución de su vector opuesto $-\boldsymbol{m}$; y se mantiene sólo aquellos armónicos tales que $\alpha_{m} \lesssim \alpha_{1} / 23^{2}$.

Como los vectores armónicos a $\mathcal{O}(\epsilon)$ hasta $\mathcal{O}\left(1 / 23^{2}\right)$ pueden ser separados en dos grupos, se denota con $\mathcal{Y}$ al subconjunto de vectores cuya tercera componente es nula y con $\mathcal{Z}$ a aquellos vectores que tienen su segunda componente nula. Por lo tanto, la perturbación puede ser escrita en la forma:

$$
V(\boldsymbol{I}, \boldsymbol{\theta})=\hat{V}_{12}(\boldsymbol{I}) \sum_{\boldsymbol{m} \in \mathcal{Y}} \alpha_{\boldsymbol{m}} \cos (\boldsymbol{m} \cdot \theta)+\hat{V}_{13}(\boldsymbol{I}) \sum_{\boldsymbol{m} \in \mathcal{Z}} \alpha_{\boldsymbol{m}} \cos (\boldsymbol{m} \cdot \theta) .
$$

Al aplicar la condición de resonancia, $\boldsymbol{m} \cdot \boldsymbol{\omega}=0$, con $\boldsymbol{m} \in \mathbb{Z}^{3} \backslash\{\mathbf{0}\}$, al sistema no perturbado, se obtiene la siguiente relación entre las acciones en cada grado de libertad:

$$
m_{1} I_{1}^{1 / 3}+m_{2} I_{2}^{1 / 3}+m_{3} I_{3}^{1 / 3}=0,
$$

por lo que ningún vector resonante puede tener sus tres componentes del mismo signo. Además, en la perturbación no hay armónicos con dos de sus componentes iguales a cero.

Así, se obtuvieron doce vectores resonantes diferentes, agrupados en el siguiente conjunto:

$$
\begin{aligned}
\mathcal{V}_{r}\left(\epsilon, 1 / 23^{2}\right)=\{ & (2,-1,0),(2,-3,0),(2,-5,0),(4,-1,0),(4,-3,0),(6,-1,0),(6,-1,0), \\
& (2,0,-1),(2,0,-3),(2,0,-5),(4,0,-1),(4,0,-3),(6,0,-1)\}
\end{aligned}
$$

Para aquellos vectores $\boldsymbol{m} \in \mathcal{Y}$, la condición de resonancia junto con la conservación de la energía define una curva en el espacio de acciones (no sólo un punto, como en el caso 2DoF) dada por:

$$
\left\{\begin{array}{l}
I_{2}^{r}=-\left(\frac{m_{1}}{m_{2}}\right)^{3} I_{1}^{r} \\
I_{3}^{r}=\left\{\frac{h}{A}-\left(1+\frac{m_{1}^{4}}{m_{2}^{4}}\right)\left(I_{1}^{r}\right)^{4 / 3}\right\}^{3 / 4}
\end{array}\right.
$$

donde $I_{1}^{r} \in\left[0, I_{\text {max }}^{\mathcal{Y}}\right]$, siendo $I_{\text {max }}^{\mathcal{Y}}=\left(\frac{h / A}{1+\left(m_{1} / m_{2}\right)^{4}}\right)^{3 / 4}$ la cota superior.

La correspondiente curva para $\boldsymbol{m} \in \mathcal{Z}$ está dada por

$$
\left\{\begin{array}{l}
I_{2}^{r}=\left\{\frac{h}{A}-\left(1+\frac{m_{1}^{4}}{m_{3}^{4}}\right)\left(I_{1}^{r}\right)^{4 / 3}\right\}^{3 / 4} \\
I_{3}^{r}=-\left(\frac{m_{1}}{m_{3}}\right)^{3} I_{1}^{r}
\end{array}\right.
$$

con $I_{1}^{r} \in\left[0, I_{\text {max }}^{\mathcal{Z}}\right]$, con $I_{\max }^{\mathcal{Z}}=\left(\frac{h / A}{1+\left(m_{1} / m_{3}\right)^{4}}\right)^{3 / 4}$. Al aplicar la aproximación del péndulo, se obtiene un Hamiltoniano resonante similar al dado por la ecuación (3.26), es decir,

$$
H_{r}\left(p_{1}, \psi_{1}\right)=\frac{p_{1}^{2}}{2 M}+\epsilon V_{m} \cos \psi_{1}
$$

donde $V_{\boldsymbol{m}}=\hat{V}_{12}\left(\boldsymbol{I}^{\boldsymbol{r}}\right) \alpha_{\boldsymbol{m}}$ para $\boldsymbol{m} \in \mathcal{Y}$, y $V_{\boldsymbol{m}}=\hat{V}_{13}\left(\boldsymbol{I}^{\boldsymbol{r}}\right) \alpha_{\boldsymbol{m}}$ para $\boldsymbol{m} \in \mathcal{Z}$. 
En el espacio de energía, los anchos de resonancia en cada grado de libertad miden lo siguiente:

$$
\left(\Delta h_{i}\right)_{\boldsymbol{m}}^{r}=\omega_{i}\left(I_{i}^{r}\right)\left(\Delta I_{i}\right)_{\boldsymbol{m}}^{r}=\frac{4}{3} A\left(I_{i}^{r}\right)^{1 / 3} 2\left(\epsilon M V_{\boldsymbol{m}}\right)^{1 / 2} m_{i}=\frac{8}{3} A\left(I_{i}^{r}\right)^{1 / 3}\left(\epsilon M V_{\boldsymbol{m}}\right)^{1 / 2} m_{i} .
$$

Mientras que en el modelo 2DoF las condiciones de resonancia y conservación de energía fuerzan a que la resonancia dependa de sólo una variable, sea $h, h_{1}^{r}$ o $I_{1}^{r}$, en el modelo 3DoF el ancho de resonancia es una función de dos variables independientes, que se ha elegido sean $I_{1}^{r} \mathrm{y} h$.

Con los anchos computados mediante el uso de (3.49), es posible trazar los desplazamientos de la energías resonantes, $h_{i}^{r}+\left(\Delta h_{i}\right)_{m}^{r}$ y $h_{i}^{r}-\left(\Delta h_{i}\right)_{m}^{r}$, para valores de $I_{1}^{r} \in\left[0, I_{\max }\right]$. Por lo tanto, siguiendo a Cincotta et al. (2003) hemos realizado el siguiente cambio global de coordenadas:

$$
\begin{aligned}
& e_{1}=\frac{1}{\sqrt{6}}\left(h_{1}-2 h_{2}+h_{3}\right), \\
& e_{2}=\frac{1}{\sqrt{2}}\left(h_{1}-h_{3}\right), \\
& e_{3}=\frac{1}{\sqrt{3}}\left(h_{1}+h_{2}+h_{3}\right),
\end{aligned}
$$

donde $e_{1} \in\left[-\sqrt{\frac{2}{3}} h, \frac{h}{\sqrt{6}}\right], e_{2} \in\left[-\frac{h}{\sqrt{2}}, \frac{h}{\sqrt{2}}\right], e_{3}=\frac{h}{\sqrt{3}}$, adoptando el valor $h=0.485$, para finalmente mostrar en la Fig. 3.6 la región de la superficie de energía ocupada por la estructura resonante de los vectores pertenecientes a $\mathcal{V}_{r}\left(\epsilon, 1 / 23^{2}\right)$ para dos valores de $\epsilon$. Muchas de las resonancias en $\mathcal{V}_{r}\left(\epsilon, 1 / 23^{2}\right)$ son apenas observables debido a su delgadez o a su proximidad a algún borde.
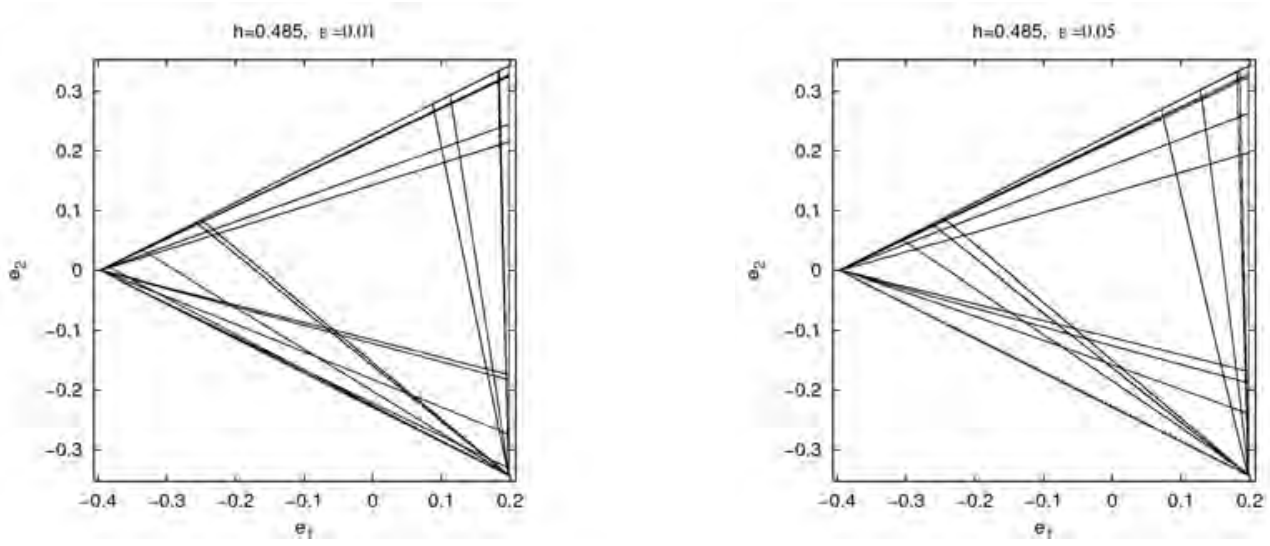

Figura 3.6: Resonancias de $\mathcal{V}_{r}\left(\epsilon, 1 / 23^{2}\right)$ para $\epsilon=0.01$ (izquierda) y $\epsilon=0.05$ (derecha).

\subsection{Resonancias a $\mathcal{O}\left(\epsilon^{2}\right)$}

Como en el caso 2DoF, se realiza una transformación canónica para remover la perturbación a $\mathcal{O}(\epsilon)$ y llevarla a $\mathcal{O}\left(\epsilon^{2}\right)$, siendo la función generatriz de la transformación de la forma: 


$$
F(\boldsymbol{J}, \boldsymbol{\theta})=\boldsymbol{J} \cdot \boldsymbol{\theta}+\epsilon \Phi(\boldsymbol{J}, \boldsymbol{\theta}),
$$

donde

$$
\Phi(\boldsymbol{J}, \boldsymbol{\theta})=\sum_{\boldsymbol{m} \in \mathcal{Y}} \Phi_{\boldsymbol{m}}(\boldsymbol{J}) \sin (\boldsymbol{m} \cdot \boldsymbol{\theta})+\sum_{\boldsymbol{m} \in \mathcal{Z}} \Phi_{\boldsymbol{m}}(\boldsymbol{J}) \sin (\boldsymbol{m} \cdot \boldsymbol{\theta}),
$$

$\operatorname{con} \Phi_{m}(\boldsymbol{J})=-V_{\boldsymbol{m}}(\boldsymbol{J}) / \boldsymbol{m} \cdot \boldsymbol{\omega}(\boldsymbol{J})$.

El nuevo Hamiltoniano esta descripto por la expresión formal dada en (3.32), donde ambos el primer y el segundo término dentro de las llaves adoptan los valores:

$$
\frac{1}{2} \frac{\partial^{2} H_{0}(\boldsymbol{J})}{\partial J_{j} \partial J_{i}} \frac{\partial \Phi(\boldsymbol{J}, \boldsymbol{\varphi})}{\partial \varphi_{j}} \frac{\partial \Phi(\boldsymbol{J}, \boldsymbol{\varphi})}{\partial \varphi_{i}}=
$$

$$
\begin{aligned}
& =\sum_{\boldsymbol{m} \in \mathcal{Y}} \sum_{\boldsymbol{m}^{\prime} \in \mathcal{Y}} \varrho_{\boldsymbol{m}, \boldsymbol{m}^{\prime}} \frac{\left(m_{1} m_{1}^{\prime} J_{1}^{2 / 3} J_{2}^{2 / 3}+m_{2} m_{2}^{\prime} J_{1}^{4 / 3}\right)}{\left(m_{1} J_{1}^{1 / 3}+m_{2} J_{2}^{1 / 3}\right)\left(m_{1}^{\prime} J_{1}^{1 / 3}+m_{2}^{\prime} J_{2}^{1 / 3}\right)}\left\{\cos \left(\left(\boldsymbol{m}+\boldsymbol{m}^{\prime}\right) \cdot \boldsymbol{\varphi}\right)+\cos \left(\left(\boldsymbol{m}-\boldsymbol{m}^{\prime}\right) \cdot \boldsymbol{\varphi}\right)\right\}+ \\
& +\sum_{\boldsymbol{m} \in \mathcal{Z}} \sum_{\boldsymbol{m}^{\prime} \in \mathcal{Z}} \varrho_{\boldsymbol{m}, \boldsymbol{m}^{\prime}} \frac{\left(m_{1} m_{1}^{\prime} J_{1}^{2 / 3} J_{3}^{2 / 3}+m_{3} m_{3}^{\prime} J_{1}^{4 / 3}\right)}{\left(m_{1} J_{1}^{1 / 3}+m_{3} J_{3}^{1 / 3}\right)\left(m_{1}^{\prime} J_{1}^{1 / 3}+m_{3}^{\prime} J_{3}^{1 / 3}\right)}\left\{\cos \left(\left(\boldsymbol{m}+\boldsymbol{m}^{\prime}\right) \cdot \boldsymbol{\varphi}\right)+\cos \left(\left(\boldsymbol{m}-\boldsymbol{m}^{\prime}\right) \cdot \boldsymbol{\varphi}\right)\right\}+ \\
& +\sum_{\boldsymbol{m} \in \mathcal{Y}} \sum_{\boldsymbol{m}^{\prime} \in \mathcal{Z}} \varrho_{\boldsymbol{m}, \boldsymbol{m}^{\prime}} \frac{m_{1} m_{1}^{\prime} J_{1}^{2 / 3} J_{2}^{1 / 3} J_{3}^{1 / 3}}{\left(m_{1} J_{1}^{1 / 3}+m_{2} J_{2}^{1 / 3}\right)\left(m_{1}^{\prime} J_{1}^{1 / 3}+m_{3}^{\prime} J_{3}^{1 / 3}\right)}\left\{\cos \left(\left(\boldsymbol{m}+\boldsymbol{m}^{\prime}\right) \cdot \boldsymbol{\varphi}\right)+\cos \left(\left(\boldsymbol{m}-\boldsymbol{m}^{\prime}\right) \cdot \boldsymbol{\varphi}\right)\right\}+ \\
& +\sum_{\boldsymbol{m} \in \mathcal{Z}} \sum_{\boldsymbol{m}^{\prime} \in \mathcal{Y}} \varrho_{\boldsymbol{m}, \boldsymbol{m}^{\prime}} \frac{m_{1} m_{1}^{\prime} J_{1}^{2 / 3} J_{2}^{1 / 3} J_{3}^{1 / 3}}{\left(m_{1} J_{1}^{1 / 3}+m_{3} J_{3}^{1 / 3}\right)\left(m_{1}^{\prime} J_{1}^{1 / 3}+m_{2}^{\prime} J_{2}^{1 / 3}\right)}\left\{\cos \left(\left(\boldsymbol{m}+\boldsymbol{m}^{\prime}\right) \cdot \boldsymbol{\varphi}\right)+\cos \left(\left(\boldsymbol{m}-\boldsymbol{m}^{\prime}\right) \cdot \boldsymbol{\varphi}\right)\right\},
\end{aligned}
$$

donde $\varrho_{\boldsymbol{m}, \boldsymbol{m}^{\prime}}=2^{3} 3^{2 / 3} \beta^{20 / 3} \alpha_{\boldsymbol{m}} \alpha_{\boldsymbol{m}^{\prime}} \mathrm{y}$

$$
\frac{\partial V(\boldsymbol{J}, \boldsymbol{\varphi})}{\partial J_{i}} \frac{\partial \Phi(\boldsymbol{J}, \boldsymbol{\varphi})}{\partial \varphi_{i}}=
$$

$$
\begin{aligned}
= & -\sum_{\boldsymbol{m} \in \mathcal{Y}} \sum_{\boldsymbol{m}^{\prime} \in \mathcal{Y}} 2 \varrho_{\boldsymbol{m}, \boldsymbol{m}^{\prime}} \frac{\left(2 m_{1}^{\prime} J_{1}^{1 / 3} J_{2}^{2 / 3}+m_{2}^{\prime} J_{1}^{4 / 3} J_{2}^{-1 / 3}\right)}{\left(m_{1}^{\prime} J_{1}^{1 / 3}+m_{2}^{\prime} J_{2}^{1 / 3}\right)}\left\{\cos \left(\left(\boldsymbol{m}+\boldsymbol{m}^{\prime}\right) \cdot \boldsymbol{\varphi}\right)+\cos \left(\left(\boldsymbol{m}-\boldsymbol{m}^{\prime}\right) \cdot \boldsymbol{\varphi}\right)\right\}+ \\
& -\sum_{\boldsymbol{m} \in \mathcal{Z}} \sum_{\boldsymbol{m}^{\prime} \in \mathcal{Z}} 2 \varrho_{\boldsymbol{m}, \boldsymbol{m}^{\prime}} \frac{\left(2 m_{1}^{\prime} J_{1}^{1 / 3} J_{3}^{2 / 3}+m_{3}^{\prime} J_{1}^{4 / 3} J_{3}^{-1 / 3}\right)}{\left(m_{1}^{\prime} J_{1}^{1 / 3}+m_{3}^{\prime} J_{3}^{1 / 3}\right)}\left\{\cos \left(\left(\boldsymbol{m}+\boldsymbol{m}^{\prime}\right) \cdot \boldsymbol{\varphi}\right)+\cos \left(\left(\boldsymbol{m}-\boldsymbol{m}^{\prime}\right) \cdot \boldsymbol{\varphi}\right)\right\}+ \\
& -\sum_{\boldsymbol{m} \in \mathcal{Y}} \sum_{\boldsymbol{m}^{\prime} \in \mathcal{Z}} 2^{2} \varrho_{\boldsymbol{m}, \boldsymbol{m}^{\prime}} \frac{m_{1}^{\prime} J_{1}^{1 / 3} J_{2}^{1 / 3} J_{3}^{1 / 3}}{\left(m_{1}^{\prime} J_{1}^{1 / 3}+m_{3}^{\prime} J_{3}^{1 / 3}\right)}\left\{\cos \left(\left(\boldsymbol{m}+\boldsymbol{m}^{\prime}\right) \cdot \boldsymbol{\varphi}\right)+\cos \left(\left(\boldsymbol{m}-\boldsymbol{m}^{\prime}\right) \cdot \boldsymbol{\varphi}\right)\right\}+ \\
& -\sum_{\boldsymbol{m} \in \mathcal{Z}} \sum_{\boldsymbol{m}^{\prime} \in \mathcal{Y}} 2^{2} \varrho_{\boldsymbol{m}, \boldsymbol{m}^{\prime}} \frac{m_{1}^{\prime} J_{1}^{1 / 3} J_{2}^{1 / 3} J_{3}^{1 / 3}}{\left(m_{1}^{\prime} J_{1}^{1 / 3}+m_{2}^{\prime} J_{2}^{1 / 3}\right)}\left\{\cos \left(\left(\boldsymbol{m}+\boldsymbol{m}^{\prime}\right) \cdot \boldsymbol{\varphi}\right)+\cos \left(\left(\boldsymbol{m}-\boldsymbol{m}^{\prime}\right) \cdot \boldsymbol{\varphi}\right)\right\},
\end{aligned}
$$


Por lo tanto, el Hamiltoniano puede ser reescrito como:

$$
\begin{aligned}
\mathcal{H}(\boldsymbol{J}, \boldsymbol{\varphi})=H_{0}(\boldsymbol{J})+\epsilon^{2} \sum_{n \in \mathcal{A}}\{ & \sum_{\boldsymbol{m} \in \mathcal{Y}} \sum_{\boldsymbol{m}^{\prime} \in \mathcal{Y}}\left[a\left(\boldsymbol{n}, \boldsymbol{m}, \boldsymbol{m}^{\prime}, \boldsymbol{J}\right)+a\left(\boldsymbol{n}, \boldsymbol{m},-\boldsymbol{m}^{\prime}, \boldsymbol{J}\right)\right] \cos (\boldsymbol{n} \cdot \boldsymbol{\varphi})+ \\
& +\sum_{\boldsymbol{m} \in \mathcal{Z}} \sum_{\boldsymbol{m}^{\prime} \in \mathcal{Z}}\left[b\left(\boldsymbol{n}, \boldsymbol{m}, \boldsymbol{m}^{\prime}, \boldsymbol{J}\right)+b\left(\boldsymbol{n}, \boldsymbol{m},-\boldsymbol{m}^{\prime}, \boldsymbol{J}\right)\right] \cos (\boldsymbol{n} \cdot \boldsymbol{\varphi})+ \\
& +\sum_{\boldsymbol{m} \in \mathcal{Y}} \sum_{\boldsymbol{m}^{\prime} \in \mathcal{Z}}\left[c\left(\boldsymbol{n}, \boldsymbol{m}, \boldsymbol{m}^{\prime}, \boldsymbol{J}\right)+c\left(\boldsymbol{n}, \boldsymbol{m},-\boldsymbol{m}^{\prime}, \boldsymbol{J}\right)\right] \cos (\boldsymbol{n} \cdot \boldsymbol{\varphi})+ \\
& \left.+\sum_{\boldsymbol{m} \in \mathcal{Z}} \sum_{\boldsymbol{m}^{\prime} \in \mathcal{Y}}\left[d\left(\boldsymbol{n}, \boldsymbol{m}, \boldsymbol{m}^{\prime}, \boldsymbol{J}\right)+d\left(\boldsymbol{n}, \boldsymbol{m},-\boldsymbol{m}^{\prime}, \boldsymbol{J}\right)\right] \cos (\boldsymbol{n} \cdot \boldsymbol{\varphi})\right\},
\end{aligned}
$$

donde $\mathcal{A}$ es un conjunto de vectores armónicos que aparecen a través de cualquier combinación de vectores de $\mathcal{Y} \cup \mathcal{Z}$ y cuya primer componente no nula es positiva, y donde los coeficientes $a, b, c$, y $d$ están definidos como sigue:

$$
\begin{aligned}
& a\left(\boldsymbol{n}, \boldsymbol{m}, \boldsymbol{m}^{\prime}, \boldsymbol{J}\right)=\varrho_{\boldsymbol{m}, \boldsymbol{m}^{\prime}}\left[\delta\left(\boldsymbol{m}+\boldsymbol{m}^{\prime}-\boldsymbol{n}\right)+\delta\left(\boldsymbol{m}+\boldsymbol{m}^{\prime}+\boldsymbol{n}\right)\right] \\
& \times\left[\frac{\left(m_{1} m_{1}^{\prime} J_{1}^{2 / 3} J_{2}^{2 / 3}+m_{2} m_{2}^{\prime} J_{1}^{4 / 3}\right)}{\left(m_{1} J_{1}^{1 / 3}+m_{2} J_{2}^{1 / 3}\right)\left(m_{1}^{\prime} J_{1}^{1 / 3}+m_{2}^{\prime} J_{2}^{1 / 3}\right)}-2 \frac{\left(2 m_{1}^{\prime} J_{1}^{1 / 3} J_{2}^{2 / 3}+m_{2}^{\prime} J_{1}^{4 / 3} J_{2}^{-1 / 3}\right)}{\left(m_{1}^{\prime} J_{1}^{1 / 3}+m_{2}^{\prime} J_{2}^{1 / 3}\right)}\right] . \\
& b\left(\boldsymbol{n}, \boldsymbol{m}, \boldsymbol{m}^{\prime}, \boldsymbol{J}\right)=\varrho_{\boldsymbol{m}, \boldsymbol{m}^{\prime}}\left[\delta\left(\boldsymbol{m}+\boldsymbol{m}^{\prime}-\boldsymbol{n}\right)+\delta\left(\boldsymbol{m}+\boldsymbol{m}^{\prime}+\boldsymbol{n}\right)\right] \\
& \times\left[\frac{\left(m_{1} m_{1}^{\prime} J_{1}^{2 / 3} J_{3}^{2 / 3}+m_{3} m_{3}^{\prime} J_{1}^{4 / 3}\right)}{\left(m_{1} J_{1}^{1 / 3}+m_{3} J_{3}^{1 / 3}\right)\left(m_{1}^{\prime} J_{1}^{1 / 3}+m_{3}^{\prime} J_{3}^{1 / 3}\right)}-2 \frac{\left(2 m_{1}^{\prime} J_{1}^{1 / 3} J_{3}^{2 / 3}+m_{3}^{\prime} J_{1}^{4 / 3} J_{3}^{-1 / 3}\right)}{\left(m_{1}^{\prime} J_{1}^{1 / 3}+m_{3}^{\prime} J_{3}^{1 / 3}\right)}\right] . \\
& c\left(\boldsymbol{n}, \boldsymbol{m}, \boldsymbol{m}^{\prime}, \boldsymbol{J}\right)=\varrho_{\boldsymbol{m}, \boldsymbol{m}^{\prime}}\left[\delta\left(\boldsymbol{m}+\boldsymbol{m}^{\prime}-\boldsymbol{n}\right)+\delta\left(\boldsymbol{m}+\boldsymbol{m}^{\prime}+\boldsymbol{n}\right)\right] \\
& \times\left[\frac{m_{1} m_{1}^{\prime} J_{1}^{2 / 3} J_{2}^{1 / 3} J_{3}^{1 / 3}}{\left(m_{1} J_{1}^{1 / 3}+m_{2} J_{2}^{1 / 3}\right)\left(m_{1}^{\prime} J_{1}^{1 / 3}+m_{3}^{\prime} J_{3}^{1 / 3}\right)}-4 \frac{m_{1}^{\prime} J_{1}^{1 / 3} J_{2}^{1 / 3} J_{3}^{1 / 3}}{\left(m_{1}^{\prime} J_{1}^{1 / 3}+m_{3}^{\prime} J_{3}^{1 / 3}\right)}\right] . \\
& d\left(\boldsymbol{n}, \boldsymbol{m}, \boldsymbol{m}^{\prime}, \boldsymbol{J}\right)=\varrho_{\boldsymbol{m}, \boldsymbol{m}^{\prime}}\left[\delta\left(\boldsymbol{m}+\boldsymbol{m}^{\prime}-\boldsymbol{n}\right)+\delta\left(\boldsymbol{m}+\boldsymbol{m}^{\prime}+\boldsymbol{n}\right)\right] \\
& \times\left[\frac{m_{1} m_{1}^{\prime} J_{1}^{2 / 3} J_{2}^{1 / 3} J_{3}^{1 / 3}}{\left(m_{1} J_{1}^{1 / 3}+m_{3} J_{3}^{1 / 3}\right)\left(m_{1}^{\prime} J_{1}^{1 / 3}+m_{2}^{\prime} J_{2}^{1 / 3}\right)}-4 \frac{m_{1}^{\prime} J_{1}^{1 / 3} J_{2}^{1 / 3} J_{3}^{1 / 3}}{\left(m_{1}^{\prime} J_{1}^{1 / 3}+m_{2}^{\prime} J_{2}^{1 / 3}\right)}\right] .
\end{aligned}
$$

En estas ecuaciones se ha utilizado la función $\delta$-Kronecker (vectorial), definida como

$$
\delta(\boldsymbol{m})= \begin{cases}1 & \text { si } \boldsymbol{m}=0 \\ 0 & \text { si } \boldsymbol{m} \neq 0\end{cases}
$$


Además, introduciendo un coeficiente análogo al usado en la perturbación a $\mathcal{O}\left(\epsilon^{2}\right)$ para el modelo 2DoF,

$$
\begin{aligned}
\mathbb{D}(\boldsymbol{n}, \boldsymbol{J}) & =\sum_{\boldsymbol{m} \in \mathcal{Y}} \sum_{\boldsymbol{m}^{\prime} \in \mathcal{Y}}\left[a\left(\boldsymbol{n}, \boldsymbol{m}, \boldsymbol{m}^{\prime}, \boldsymbol{J}\right)+a\left(\boldsymbol{n}, \boldsymbol{m},-\boldsymbol{m}^{\prime}, \boldsymbol{J}\right)\right] \\
& +\sum_{\boldsymbol{m} \in \mathcal{Z}} \sum_{\boldsymbol{m}^{\prime} \in \mathcal{Z}}\left[b\left(\boldsymbol{n}, \boldsymbol{m}, \boldsymbol{m}^{\prime}, \boldsymbol{J}\right)+b\left(\boldsymbol{n}, \boldsymbol{m},-\boldsymbol{m}^{\prime}, \boldsymbol{J}\right)\right] \\
& +\sum_{\boldsymbol{m} \in \mathcal{Y}} \sum_{\boldsymbol{m}^{\prime} \in \mathcal{Z}}\left[c\left(\boldsymbol{n}, \boldsymbol{m}, \boldsymbol{m}^{\prime}, \boldsymbol{J}\right)+c\left(\boldsymbol{n}, \boldsymbol{m},-\boldsymbol{m}^{\prime}, \boldsymbol{J}\right)\right] \\
& +\sum_{\boldsymbol{m} \in \mathcal{Z}} \sum_{\boldsymbol{m}^{\prime} \in \mathcal{Y}}\left[d\left(\boldsymbol{n}, \boldsymbol{m}, \boldsymbol{m}^{\prime}, \boldsymbol{J}\right)+d\left(\boldsymbol{n}, \boldsymbol{m},-\boldsymbol{m}^{\prime}, \boldsymbol{J}\right)\right]
\end{aligned}
$$

el Hamiltoniano admite la expresión:

$$
\mathcal{H}(\boldsymbol{J}, \boldsymbol{\varphi})=H_{0}(\boldsymbol{J})+\epsilon^{2} \sum_{\boldsymbol{n} \in \mathcal{A}} \mathbb{D}(\boldsymbol{n}, \boldsymbol{J}) \cos (\boldsymbol{n} \cdot \boldsymbol{\varphi}) .
$$

Cerca de cualquier acción resonante asociada a un vector resonante a $\mathcal{O}\left(\epsilon^{2}\right)$ se puede aplicar la aproximación del péndulo para obtener la misma expresión formal para el Hamiltoniano resonante dado por (3.37).

Las variaciones de las energías son similares a aquellas computadas a $\mathcal{O}(\epsilon)$; es decir,

$$
\Delta h_{i}=\frac{8}{3} A J_{i}^{1 / 3}\left(\mu\left|\mathcal{U}_{0}\right|\right)^{1 / 2} n_{i} .
$$

Para aquellos $\boldsymbol{n}$ con $n_{3}=0$ o $n_{2}=0$, las curvas resonantes están dadas por las ecuaciones (3.46) y (3.47) respectivamente, con la substitución de $m_{i}$ y $I_{i}$ por $n_{i}$ y $J_{i}$. Mientras tanto, cuando $n_{1}=0$ las curvas resonantes están dadas por

$$
\left\{\begin{array}{l}
J_{2}^{r}=\frac{1}{\left(1+\left(n_{2} / n_{3}\right)^{4}\right)^{3 / 4}}\left\{\frac{h}{A}-\left(J_{1}^{r}\right)^{4 / 3}\right\}^{3 / 4}, \\
J_{3}^{r}=\frac{1}{\left(1+\left(n_{3} / n_{2}\right)^{4}\right)^{3 / 4}}\left\{\frac{h}{A}-\left(J_{1}^{r}\right)^{4 / 3}\right\}^{3 / 4},
\end{array}\right.
$$

con $J_{1}^{r} \in\left[0,\left(\frac{h}{A}\right)^{3 / 4}\right]$.

Sea $\mathcal{V}_{r}\left(\epsilon^{2}, 1 / 23^{2}\right)$ el conjunto de vectores resonantes que pertenecen a $\mathcal{A}$ y que pueden ser construídos por al menos un par $\left(\boldsymbol{m}, \boldsymbol{m}^{\prime}\right)$ tal que $\alpha_{\boldsymbol{m}} \alpha_{\boldsymbol{m}^{\prime}} \lesssim \alpha_{1}^{6} / 23^{2}$.

Al computar los elementos de $\mathcal{V}_{r}\left(\epsilon^{2}, 1 / 23^{2}\right)$ se observa que este conjunto consiste de 15 vectores con una componente nula junto con 48 vectores que poseen sus tres componentes distintas a cero.

La Fig. 3.7 muestra el area de la superficie de energía canónica $(h=0.485)$ ocupada por aquellas resonancias pertenencientes a $\mathcal{V}_{r}\left(\epsilon^{2}, 1 / 23^{2}\right)$ que poseen una componente nula más la resonancia $(2,-1,-1)$, para los mismo dos valores del parámetro perturbativo usados en la Fig. 3.6.

Tal subconjunto de $\mathcal{V}_{r}\left(\epsilon^{2}, 1 / 23^{2}\right)$, así como también el conjunto completo $\mathcal{V}_{r}\left(\epsilon, 1 / 23^{2}\right)$ han sido considerados en la Fig. 3.8-izquierda para $\epsilon=0.005$.

Esta imagen puede ser comparada con el mapa de niveles del promedio temporal del MEGNO, para el mismo valor de $\epsilon$, que se muestra en la Fig. 3.8-derecha ${ }^{2}$. Para confeccionar

\footnotetext{
${ }^{2}$ Material perteneciente a Cincotta et al. (2003)
} 

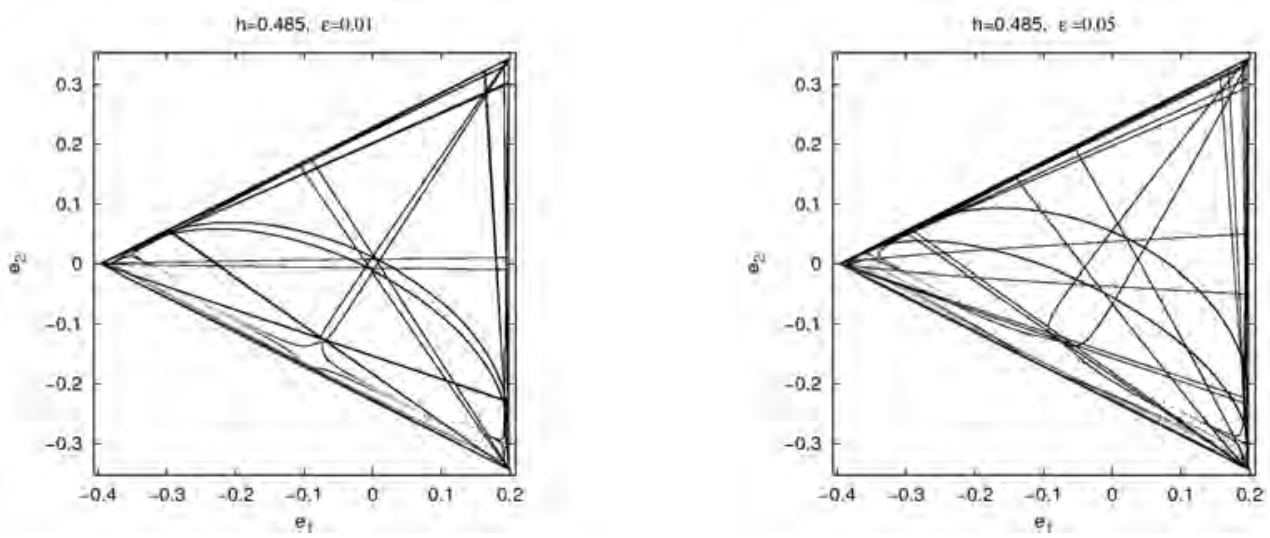

Figura 3.7: Resonancias de $\mathcal{V}_{r}\left(\epsilon^{2}, 1 / 23^{2}\right)$ para $\epsilon=0.01$ (izquierda) y $\epsilon=0.05$ (derecha).

dicho mapa, los valores de $\bar{Y}\left(t_{T}\right)$ (para $t_{T}$ igual a 3500 periodos característicos) fueron agrupados en cinco intervalos: $[1.99,1.995),[2,2.015),[2.015,20),[20,160),[160,215)$. Al asignar un tamaño de punto distinto a cada intervalo, se simuló una escala de grises del mencionado indicador.

Esta exploración numérica evidencia que las resonancias que más fuertemente se manifiestan son aquellas con sólo una componente nula (las rectas) y la resonancia $(2,-1,-1)$, que es la resonancia con forma curva más ancha.

Dicho mapa fue confeccionado con condiciones iniciales tales que los ángulos $\left\{\theta_{i}\right\}_{i=1,2,3}$ sean iguales a $\pi / 2$, por lo que la correspondiente condición inicial de la fase resonante asociada a una dada resonancia $\mathbf{m}$ es de la forma $\psi_{1}=\left(m_{1}+m_{2}+m_{3}\right) \pi / 2$. Observando Ec. 3.28 se tiene que, debido a la dependencia con la fase resonante de la separatriz $\left(\psi_{s}\right)$, el verdadero ancho medido con mapa de caoticidad puede variar respecto del teórico debido a un factor que será igual a $\sin (\pi / 4) \approx 0.7$ si $m_{1}+m_{2}+m_{3}$ es un número impar y será igual a $\sin (\pi / 2)=1$ en caso contrario. A los fines de determinar un valor global de $\epsilon_{c}$, estas correcciones no fueron consideradas en este capítulo ${ }^{3}$.

Observando la Fig. 3.8-izquierda se revela que en algunas intersecciones entre las resonancias a $\mathcal{O}(\epsilon)$ y a $\mathcal{O}\left(\epsilon^{2}\right)$, los anchos de éstas últimas tienden asintóticamente a infinito. Esto es debido a la emergencia de pequeños denominadores en los coeficientes de Fourier de la perturbación, un hecho que nos recuerda que la transformación canónica realizada para eliminar los términos perturbativos proporcionales a $\epsilon$ no es válida en el entorno de ninguna resonancia a $\mathcal{O}(\epsilon)$ (sección 1.2.4).

Un ejemplo de este comportamiento es la intersección entre las resonancias $(0,1,-1)$ y $(2,-1,0)$. La primera es una resonancia a $\mathcal{O}\left(\epsilon^{2}\right)$ que se inicia en la esquina superior derecha de la superficie de energía y cruza por la mitad de la misma mientras que la segunda es de $\mathcal{O}(\epsilon)$ y puede ser identificada como la más ancha de las resonancias que parten desde la esquina inferior derecha de la superficie de energía.

\footnotetext{
${ }^{3}$ Estas correcciones sí serán consideradas en el capítulo 4, donde será necesario establecer condiciones iniciales con precisión en la capa estocástica de una resonancia
} 

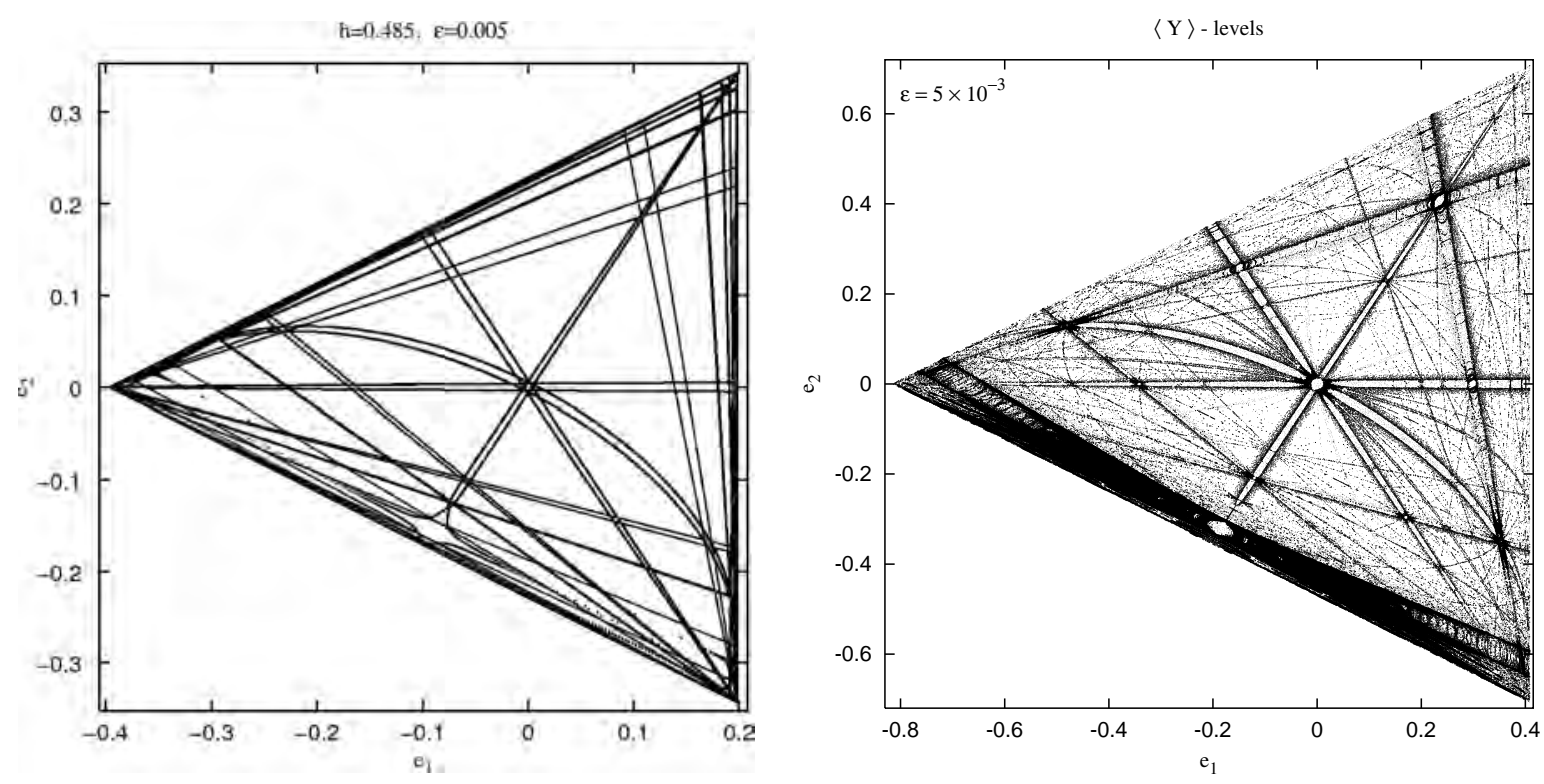

Figura 3.8: Resonancias hasta $\mathcal{O}\left(\epsilon^{2}, 1 / 23^{2}\right)$ para $\epsilon=0.005$ (izquierda). Estructura resonante obtenida al agrupar los valores de $\bar{Y}(3500 \times 2 \pi)$ en los intervalos $[1.99,1.995),[2,2.015)$, $[2.015,20),[20,160)$ y $[160,215)$, asignando un tamaño de punto distinto a cada uno. Se utilizó la misma energía, pero las variables $e_{1}, e_{2}$ fueron reescaleadas posteriormente (derecha).

\subsection{Estimación analítica del valor crítico del parámetro per- turbativo para el sistema $3 \mathrm{DoF}$}

Como puede verse de la Fig. 3.8, muchas resonancias poseen forma triangular. Tal es el caso de todas aquellas resonancias asociadas a un armónico que posee su segunda o tercera componente nula.

Puede demostrarse que para un vector resonante con su primer componente $n_{1}$ nula, los coeficientes $a\left(\boldsymbol{n}, \boldsymbol{m}, \pm \boldsymbol{m}^{\prime}, \boldsymbol{J}\right)$ y $b\left(\boldsymbol{n}, \boldsymbol{m}, \pm \boldsymbol{m}^{\prime}, \boldsymbol{J}\right)$ son nulos $\forall\left(\boldsymbol{m}, \boldsymbol{m}^{\prime}\right)$. Así, la contribución de tal vector procede sólo a través de sus correspondientes coeficientes $c\left(\boldsymbol{n}, \boldsymbol{m}, \pm \boldsymbol{m}^{\prime}, \boldsymbol{J}\right)$ y $d\left(\boldsymbol{n}, \boldsymbol{m}, \pm \boldsymbol{m}^{\prime}, \boldsymbol{J}\right)$. Además, de la ecuación (3.58) puede establecerse que el ancho de resonancia tiende a cero cuando $J_{1}$ se acerca a 0 y cuando se acerca a $(h / A)^{3 / 4}$. Consecuentemente, las regiones pertenecientes a separatrices de resonancias con $n_{1}=0$ no poseen forma triangular.

$\mathrm{Al}$ estimar $\epsilon_{\mathrm{c}}$ para el modelo $3 \mathrm{DoF}$, se han hecho las siguientes simplificaciones fuertes:

(i) se toma como $\epsilon_{\mathrm{c}}$ al valor de $\epsilon$ para el cual el área total cubierta por regiones resonantes, $\left(A_{r}\right)$, es igual al $90 \%$ de toda el área de la superficie de energía, que notaremos $\left(A_{h}\right)$. Este método está parcialmente motivado por el utilizado en sección 4.5 de Chirikov (1979). (ii) las regiones resonantes que corresponden a vectores resonantes con una sola componente nula son aproximadas por triángulos, salvo aquellas con $n_{1}=0$, que no se consideran. (iii) la región correspondiente a la resonancia $(2,-1,-1)$ es aproximada por dos triángulos. (iv) no se consideran resonancias adicionales. (v) se ha sumado el área de cada resonancia, independientemente de las intersecciones debidas a cruces u overlap, por lo que aquella area correspondientes simultáneamente a dos resonancias diferentes son consideradas dos veces.

En la Fig. 3.9-izquierda hemos graficado la fracción $A_{r} / A_{h}$ para el parámetro perturbativo variando en el rango $\epsilon \in[0.00001,0.2]$. Allí se puede observar que $A_{r}(\epsilon)$ alcanza el $90 \%$ de 
$A_{h}$ para algún $\epsilon_{\mathrm{c}}$ entre 0.03 y 0.04 . Este resultado está en acuerdo con el obtenido en la Fig. 3.9-derecha ${ }^{4}$ que muestra la fracción de movimiento caótico según valores de $\bar{Y}$, para el mismo rango de $\epsilon$.
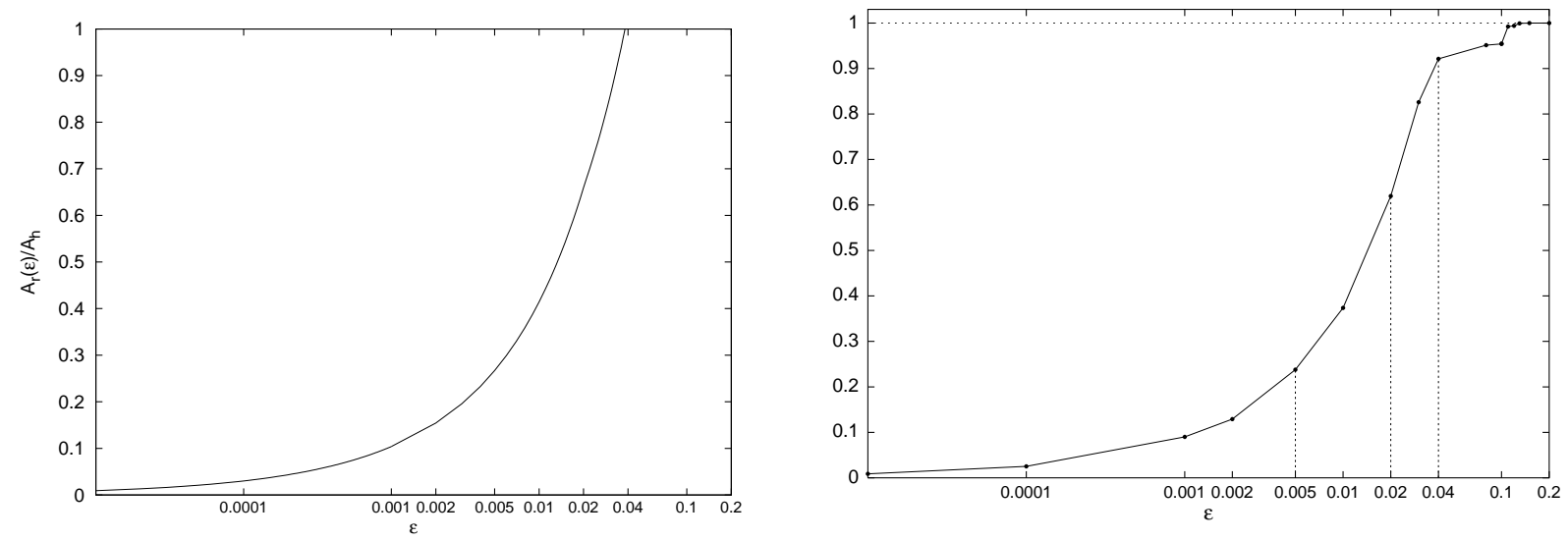

Figura 3.9: $A_{r} / A_{h}$ (izquierda) y fracción de movimiento caótico (derecha), ambos contra el parámetro perturbativo en escala logarítmica

\subsection{Conclusión}

Se ha revisado la precisión del criterio de overlap, al aplicarse a un sistema Hamiltoniano casi-integrable simple en ambas versiónes $2 \mathrm{DoF}$ y 3 DoF. Para este fin, se han computado las resonancias hasta orden $\mathcal{O}\left(\epsilon^{2}\right)$ para ambos sistemas, y modelado cada resonancia mediante la aproximación del péndulo de forma tal de estimar el valor crítico del parámetro perturbativo que determina la transición entre los regímenes de Nekhoroshev y de Chirikov.

$\mathrm{Al}$ realizar varias superficies de sección para el caso $2 \mathrm{DoF}$ se ha derivado un valor empírico de $\epsilon_{c}$ que fue comparado con la estimación teórica. Existe una diferencia significativa entre ambos valores.

Para el caso 3DoF se obtuvo un estimación teórica de $\epsilon_{c}$, que se muestra en acuerdo con la dada en Giordano y Cincotta (2004), donde tal valor es logrado computando la fracción de órbitas caóticas contra $\epsilon$ de acuerdo a los valores de $\bar{Y}$.

Debe aclararse que el concepto de transición global al caos utilizada para la estimación numérica de $\epsilon_{\mathrm{c}}$ en el caso $2 \mathrm{DoF}$ es de una naturaleza diferente respecto a la adoptada para el caso 3DoF. El sistema con 2DoF es considerado globalmente caótico si la componente caótica del espacio de fases aparece casi toda conectada, mientras que en el caso con 3DoF, el sistema es considerado como globalmente caótico cuando a lo sumo el $10 \%$ de la superficie de energía corresponde a toros invariantes.

Por lo tanto, considerando los resultados, tanto numéricos como teóricos, de este capítulo, se estableció un valor adecuado de $\epsilon_{c}$ para el modelo $3 \mathrm{DoF}$, mediante el uso del criterio de overlap, considerando algunas de las resonancias hasta $\mathcal{O}\left(\epsilon^{2}\right)$. Por otro lado, considerando términos sólo hasta $\mathcal{O}(\epsilon)$, se sobreestima el valor de $\epsilon_{c}$, como ya fue mostrado en Chirikov (1979) para el mapa estándar.

\footnotetext{
${ }^{4}$ Material perteneciente a Giordano y Cincotta (2004)
} 


\section{Fenómeno difusivo en sistema Hamiltoniano 3DoF}

En este capítulo se estudiará, mediante experimentos numéricos, cómo se manifiesta la difusión a lo largo de la separatriz perturbada de una resonancia del sistema Hamiltoniano 3DoF casi-integrable analizado en el capítulo anterior. Se trabajará con el mismo valor de $h$.

En la sección 4.1 se describe el integrador simpléctico utilizado, justificando brevemente tal elección. En la sección 4.2 se detallan los parámetros del sistema y la resonancia guía elegida, así como también las características de su entorno como por ejemplo qué resonancias vecinas posee. En las secciones 4.3, 4.4 y 4.5 se describe los experimentos realizados con ensambles de partículas prueba, analizando la forma funcional de la evolución temporal de algunas cantidades estadísticas. En dichas secciones se brindará información sobre la difusión que tiene lugar en la capa estocástica de la resonancia guía antes definida. Los mediciones realizadas pueden agruparse en tres experimentos numéricos, a los que llamaremos experimentos $\mathscr{A}, \mathscr{B}$ y $\mathscr{C}$. En el primero de ellos se trabaja con una acción resonante fija, para luego estudiar la difusión para distintos valores de $\epsilon$. En el segundo experimento se vuelven a realizar mediciones para una acción resonante fija, y para distintos valores de $\epsilon$, con la diferencia de que se utiliza un rango más amplio que el estudiado en $\mathscr{A}$. En el tercer experimento se fija el valor de $\epsilon$ de forma tal que el sistema esté en régimen de Nekhoroshev y se estudia la difusión para ensambles correspondientes a distintas acciones resonantes, ubicadas a lo largo de la resonancia guía. En todos los caso se hacen estimaciones de coeficientes de difusión.

\subsection{El integrador simpléctico}

En este capítulo, el integrador utilizado para computar las trayectorias con las que se realizaron los mapas SALI y las evoluciones de las cantidades estadísticas de ensambles de partículas prueba es un integrador simpléctico elaborado por E. Teloy, Freiburg, según mención en Schlier y Seiter (2000). Dicha subrutina integradora consiste de cinco integradores distintos, dos de orden 6 y tres de orden 8, llamados respectivamente S6a, S6b, S8a, S8b y S8c. El núcleo del código fuente, aquel que realiza la integración de un paso simpléctico, es llamado testsymp ${ }^{1}$ y fue escrito en lenguaje Fortran 90 . Dicho código contiene los coeficientes para los cinco esquemas de integración con 35 decimales, lo cual es suficiente para cómputos de hasta cuádruple precisión. Todos los integradores requieren que la energía cinética, $T$, sea separable del potencial, $V$. Es decir, requieren que el Hamiltoniano sea de la forma

\footnotetext{
${ }^{1}$ Disponible en CPC Program Library, Queen's University of Belfast, N. Ireland.
} 
$H=T(\mathbf{p})+V(\mathbf{q})$. A su vez, todos estos esquemas salvo el S6a, requieren que la energía cinética sea una forma cuadrática de los momentos. Por lo tanto, utilizando variables cartesianas para la integración de las ecuaciones de movimiento generadas por nuestro Hamiltoniano (3.39), cualquiera de estos esquemas es aplicable. En Schlier and Seiter (1998) se pone a prueba la precisión en la conservación de la energía del esquema $S 6 a$. Posteriormente, en Schlier y Seiter (2000) se prueban intensamente los cinco esquemas, trabajando con aritméticas de precisión doble y cuádruple, y midiendo la precisión con el error cometido en la posición final en el espacio de fases (y en el de configuraciones) a un tiempo fijo dado. Ellos señalan que estos integradores son precisos en este sentido, además de en la conservación de la energía. Además, afirman que los integradores de orden ocho son muy poderosos, especialmente para trayectorias que se hallan atrapadas en las capas estocásticas del espacio de fases. Justamente, ésta es la situación de las órbitas que nos interesa integrar para estudiar la difusión que tiene lugar en la red de Arnold. Dicho estudio requiere precisión y velocidad para poder rastrear las trayectorias de un número considerable de partículas de forma tal que sus propiedades estadísticas sean significativas durante intervalos de tiempo de más de una decena de millones de períodos característicos del sistema.

Previo a la utilización de este integrador para esta investigación, se realizaron varias comparaciones. Se comparó el desempeño de estos cinco integradores simplécticos junto con el DOPRI8 (Prince y Dormand, 1981), que es un integrador Runge-Kutta de orden siete con control de paso. Estos códigos se probaron usando tanto sistemas Hamiltonianos integrables como no integrables, y en estos últimos, usando tanto órbitas caóticas como regulares. Las cantidades medidas en la comparación fueron el grado de conservación de la energía, los tiempos de cpu utilizados y el número de veces que se computaron las derivadas del Hamiltoniano. La conclusión fue que los dos integradores simplécticos de orden ocho resultaron ser los más precisos respecto a la conservación de la energía. Para un mismo valor de tiempo final, los integradores de orden ocho calculan más derivadas que los de orden seis pero muchas menos que el esquema DOPRI8. Los tiempos de cpu utilizados resultaron ser proporcionales al número de evaluaciones de las derivadas, como era de esperarse. De los tres integradores simplécticos de orden ocho se eligió el llamado $S 8 b$, por su precisión en la conservación de la energía. Cabe ramarcar que respecto al cómputo de cantidades estadísticas (o promedio) en ensambles, cualquiera de los integradores simplécticos es adecuado. Lo mismo sucedió al realizar mapas del indicador SALI, en el que tanto los simplécticos de orden seis como los de orden ocho dieron estructuras resonantes análogas, con diferencias entre sí casi imperceptibles.

Posteriormente, el integrador $S 8 b$ fue comparado con un integrador Taylor (Jorba y Maorong 2005, Darriba 2008). El mismo se basa en realizar desarrollos de Taylor de las órbitas mediante el uso de álgebra simbólica. En la comparación ambos mostraron acuerdo al calcular órbitas regulares individuales y al computar cantidades estadísticas de ensambles de órbitas caóticas.

Debido a que un integrador simpléctico es un método de paso temporal (denotado $\Delta t_{\text {int }}$ ), fijo, se tiene que el tamaño de éste último es un parámetro importante a determinar. Se decidió utilizar en casi todos los experimentos $\Delta t_{\text {int }}=10^{-2}$, con algunas excepciones en las que se utilizó $\Delta t_{\text {int }}=10^{-1}$. Con ambos tamaños de paso se obtuvo prácticamente la misma estructura resonante en los mapas SALI, así como también se obtuvo concordancia en órbitas caóticas hasta tiempos del órden de $10^{3}$ y en los comportamientos generales de las cantidades estadísticas. Esto es una demostración de que estos integradores simplécticos, para nuestro sistema Hamiltoniano particular y para energías del orden de $h$, utilizando pasos de integración menores o iguales a $10^{-1}$, son estables a los fines de este trabajo de tesis. Por supuesto, al 
reducir el paso en un orden de magnitud, el tiempo total de integración es aumentado en un órden de magnitud.

Existen algunos argumentos a favor de la utilización de integradores simplécticos eficientes, como los de E. Teloy, para cálculos que involucren el seguimiento de gran cantidad de órbitas durante largos tiempos de evolución. Como ya se mencionó previamente en este trabajo, el objeto de estudio de este capítulo es la dinámica generada por las ecuaciones diferenciales asociadas al Hamiltoniano (3.39). La importancia del Hamiltoniano elegido radica en que modela la estructura resonante encontrada en otros sistemas Hamiltonianos más realistas respecto a la representación de procesos dinámicos que ocurren en sistemas Astrofísicos. Sin embargo, cualquier perturbación del mismo que mantenga la estructura resonante en rasgos generales, serviría perfectamente a los fines prácticos. Y es aquí donde juega un papel fundamental el integrador simpléctico. Éste nos permite integrar órbitas exactas, salvo error de redondeo numérico, de algún sistema Hamiltoniano suficientemente "cercano" a nuestro oscilador cuártico perturbado como para presentar la misma estructura resonante. Y además, nos permite integrar con una gran economía de tiempo de cpu. Existen muchos trabajos en la literatura en los que se estudian fenómenos de difusión utilizando mapas simplécticos en vez de flujos Hamiltonianos. Si bien en algunas ocasiones, esto se realiza con la intención de estudiar específicamente la dinámica de sistemas de tiempo discreto, en otras situaciones el objetivo es puramente el de disminuir los tiempos largos de integración necesarios para un sistema de tiempo continuo. Sin embargo, esta última elección tiene un costo ya que se termina estudiando un sistema con un número de grados de libertad no entero. Entonces, los integradores simplécticos de alta precisión y velocidad pueden pensarse como una alternativa al uso de mapas simplécticos, pues un integrador simpléctico es un mapa simpléctico que prácticamente no agrega ningún grado de libertad extra y que mantiene la estructura resonante del flujo Hamiltoniano original.

Teniendo en cuenta lo expuesto en esta sección, se decidió utilizar a lo largo de los diversos experimentos con el flujo Hamiltoniano que se presentarán en este capítulo, los integradores simplécticos $S 6 a$ y $S 8 b$. Asimismo, estos dos integradores junto con el DOPRI8, serán utilizados para confeccionar mapas de indicadores de caos, que trazarán la estructura resonante del espacio de fases. Al detallar cada experimento numérico se especificará cual de ellos fue utilizado.

\subsection{La resonancia guía y su entorno}

A continuación se brindarán algunas características geométricas del espacio de fases del sistema Hamiltoniano dado por la Ec. (3.39). En particular, se definirá la resonancia guía. Tal información se ilustrará para el caso $\epsilon=0.02$, que según los resultados del capítulo anterior, es inferior al valor crítico $\left(0.3 \leq \epsilon_{c} \leq 0.4\right)$.

La Fig. 4.1 fue construída utilizando niveles del promedio temporal del MEGNO, $\bar{Y}$, para condiciones iniciales que abarcan toda la superficie de energía no perturbada. Los valores de $\bar{Y}$ fueron agrupados en cinco intervalos: [1.993, 2.005] (rojo), [2.005,5] (verde), [5, 45] (azul), $[45,150]$ (violeta) y $[150,383]$ (celeste). A diferencia del mapa de la Fig. 3.8-der. donde se utilizaron variables que son combinación lineal de las energías no perturbadas, en la Fig. 4.1 se utiliza el espacio de acciones no perturbadas. La integración de estas órbitas fue realizada con el integrador DOPRI8 durante un tiempo total $\left(t_{T}\right)$ de 3500 períodos característicos $\left(t_{T}=3500 \times 2 \pi\right.$ unidades de tiempo). En la misma se puede ver la estructura resonante en 
el espacio de acciones para una sección de los tres ángulos $\left(\left\{\theta_{i}\right\}_{i=1,2,3}\right)$ iguales a $\pi / 2$. Tal elección de los ángulos iniciales corresponde a valores iniciales de $x, y$ y $z$ nulos, según puede deducirse de la transformación biyectiva entre variables cartesianas y ángulo-acción dada en ecuaciones (3.12) y de propiedades del coseno elíptico. Es por esta razón que se verifica $\tilde{V}(\mathbf{q}(0))=\tilde{V}(\mathbf{0})=0$, implicando que

$$
\tilde{H}(\mathbf{p}(0), \mathbf{q}(0))=\tilde{H}_{0}(\mathbf{p}(0), \mathbf{0}) .
$$

Luego, el valor inicial de $I_{3}$ se despejó de la ecuación $H_{0}(\boldsymbol{I})=h$, utilizando la expresión:

$$
I_{3}(0)=\left(\frac{h}{A}-I_{1}(0)^{4 / 3}-I_{2}(0)^{4 / 3}\right)^{3 / 4},
$$

donde la constante $A$ fue definida en el capítulo anterior.

En la mencionada figura se identifica en cyan la zona de overlap de resonancias que corresponde, para otro valor de $\epsilon$, con las resonancias cercanas al borde inferior del triángulo analizado en la Fig. 3.8, del capítulo anterior. Además, se puede ver como las condiciones iniciales más regulares (puntos rojos) se ubican en el complemento de la red de Arnold, que aparece definida por el resto de los colores, asociados a órbitas caóticas.

Como se ha dicho en la sección 2.6, el indicador SALI tiende exponencialmente a cero para órbitas caóticas. A partir de ahora, en los mapas generados con la escala cromática de este indicador, se utilizará el logaritmo decimal en vez del natural mientras que el criterio de corte consistirá en asignar el valor $\log (\mathrm{SALI})=-10$ a todas aquellas órbitas con SALI $<10^{-10}$.

En la Fig. 4.2 se muestra un mapa bidimensional del mencionado indicador para una porción de la superficie de energía no perturbada, proyectada sobre el plano $\left[I_{1}, I_{2}\right]$. Para realizar esta figura se integró una grilla de $1000 \times 1000$ condiciones iniciales pertenecientes al cuadrado $\left(I_{1}, I_{2}\right) \in[0.2,0.4] \times[0.0,0.2]$ y a la misma sección de ángulos iniciales que fue utilizada en la figura anterior. Para cada condición inicial se utilizó $t_{T}=10^{4}$ unidades de tiempo, lo que corresponde con más de mil veces el período característico del sistema. El comportamiento del SALI y de la paleta de colores es tal que las órbitas más caóticas aparecen en negro mientras que las más regulares lo hacen en amarillo.

En dicha figura se pueden apreciar tres grandes resonancias junto con muchas otras de tamaño menor. La resonancia que cruza "horizontalmente" desde $\left(I_{1}, I_{2}\right) \approx(0.2,0.05)$ hasta $\left(I_{1}, I_{2}\right) \approx(0.4,0.1)$ corresponde al vector resonante $\boldsymbol{m}_{g}=(2,-3,0)$, cuya dirección es identificada mediante una flecha verde. Esta será la resonancia sobre cuya separatriz perturbada estudiaremos la difusión y que según 1.4.4 es llamada resonancia guía. La resonancia más ancha de la figura, aquella que intersecta a la resonancia guía aproximadamente en $(0.22,0.06)$, corresponde al vector resonante $\boldsymbol{m}=(2,-1,-1)$, mientras que aquella resonancia situada en el lado derecho, que intersecta a la resonancia guía aproximadamente en $(0.37,0.10)$ corresponde al vector resonante $\boldsymbol{m}=(2,0,-2)$.

Se puede apreciar que para estos valores iniciales de los ángulos, la separatriz perturbada de la resonancia guía posee un grosor mayor respecto al de sus otras dos resonancias vecinas. El espesor de esta capa estocástica no es constante a lo largo de la resonancia, sobre todo en el extremo izquierdo, donde existe un tipo de overlap debido al hecho de que el entorno de $(0.22,0.06)$ es una región resonante de multiplicidad mayor a uno.

El resto de las resonancias, de tamaño mucho más delgado, parecieran no agregar nuevas perturbaciones sobre la separatriz, salvo aquella que se hace notar desde $\left(I_{1}, I_{2}\right) \approx(0.27,0.09)$ hasta $\left(I_{1}, I_{2}\right) \approx(0.33,0.14)$. Esta resonancia genera una protuberancia adicional en la separatriz. 


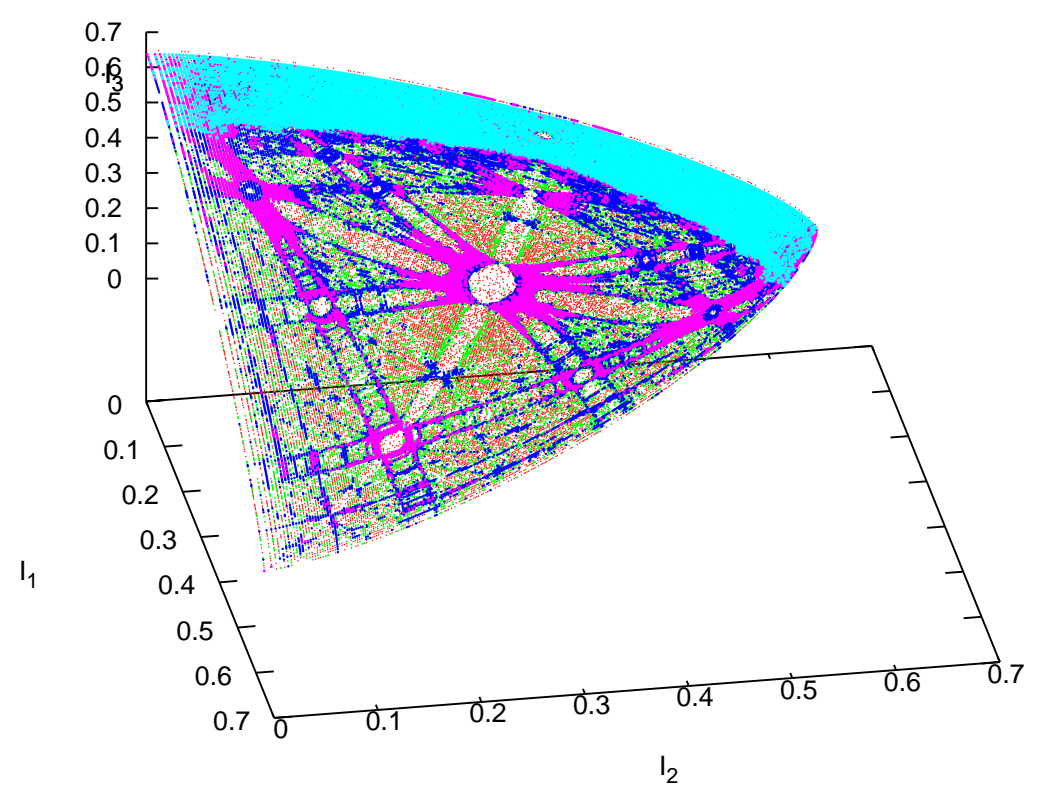

Figura 4.1: Niveles del promedio temporal del MEGNO, $\bar{Y}\left(t_{T}\right)$ con $t_{T}=3500 \times 2 \pi$, para la superficie de energía no perturbada en espacio de acciones, utilizando $\epsilon=0.02$ y $h=0.485$. La asociación entre intervalos del indicador y colores esta dada por: [1.993, 2.005]-rojo, [2.005, 5]verde, [5, 45]-azul, [45, 150]-magenta y [150,383]-cyan.

\subsection{Experimento $\mathscr{A}$}

En este experimento se estudiará la difusión para valores de $\epsilon$ pertenecientes al siguiente conjunto:

$$
\mathscr{A}_{\star}=\left\{\begin{array}{ll}
0.016,0.018,0.020,0.022,0.024,0.026,0.028,0.030
\end{array} .\right.
$$

\subsubsection{Los ensambles}

La elección de las condiciones iniciales de los ensambles se basa en la similitud de la estructura del espacio de fases en el entorno de una resonancia de multiplicidad unitaria respecto de la estructura del espacio de fases de un péndulo perturbado, de acuerdo a lo aprendido con el desarrollo perturbativo de la subsección 1.4.4. Dicha similitud será verificada gráficamente a continuación.

Consideremos la resonancia guía anteriormente definida y recordemos el cambio de variables del péndulo dado por Ec. (1.89). Ahora situémonos en un valor de la acción resonante,

$$
\boldsymbol{I}^{r}=\left(\begin{array}{l}
0.303515780714549 \\
0.089930601693199 \\
0.420802747838120
\end{array}\right)
$$

y consideremos el conjunto de condiciones iniciales que pertenecen a una porción bidimensional 


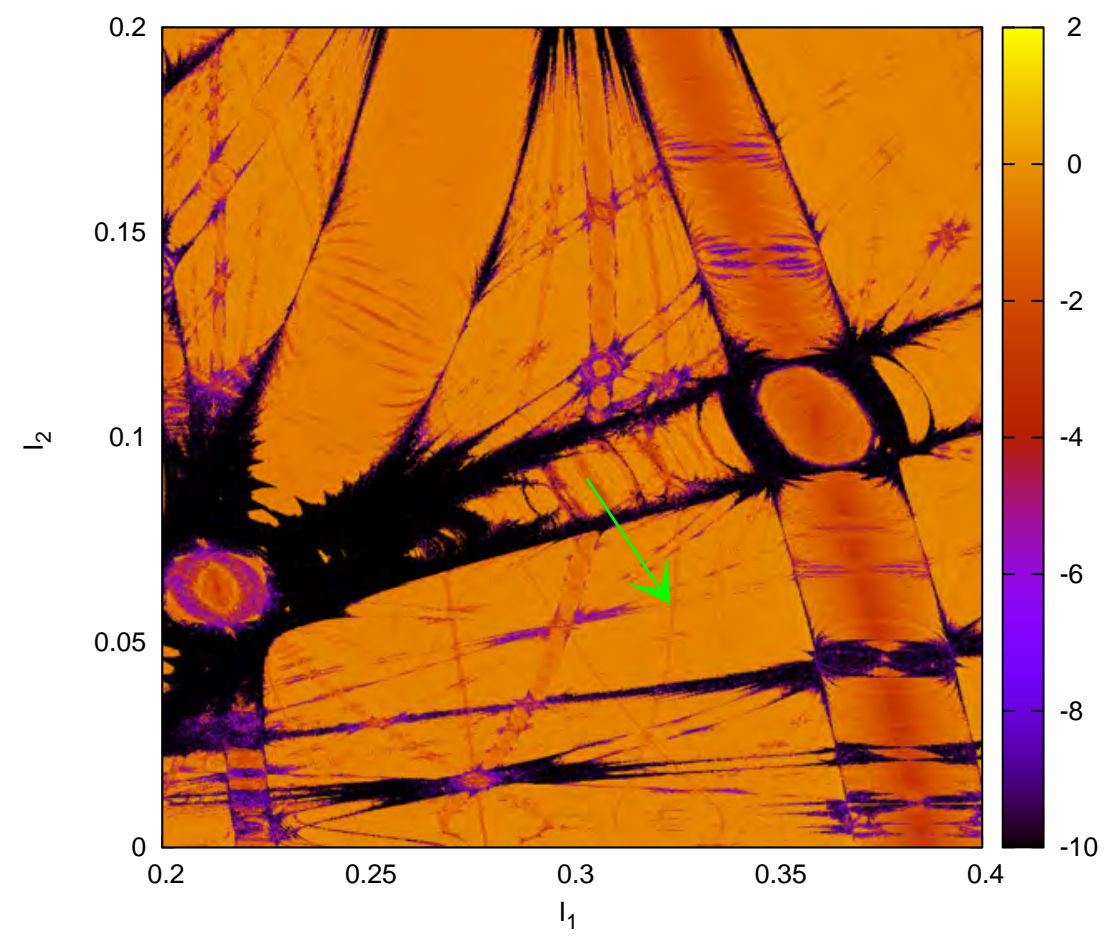

Figura 4.2: Escala cromática de $\log (\mathrm{SALI})$ en espacio de acciones; $\epsilon=0.02$ y $h$ canónico. La flecha verde es paralela al vector $\boldsymbol{m}_{g}$.

del espacio de fases parametrizada por la siguiente expresión en variables mixtas:

$$
\begin{gathered}
\left\{\begin{array}{lll}
\psi_{1} & \in[0,2 \pi] \\
p_{1} & \in\left[-10^{-2}, 10^{-2}\right]
\end{array}\right. \\
\begin{cases}\theta_{1} & =\pi / 2 \\
\theta_{2} & =\left(2 \theta_{1}-\psi_{1}\right) / 3, \quad \bmod 2 \pi \\
\theta_{3} & =\pi / 2 \\
\boldsymbol{I} & =\boldsymbol{I}^{r}+p_{1} \boldsymbol{m}_{g} .\end{cases}
\end{gathered}
$$

Así elegidas ${ }^{2}$, dichas condiciones iniciales no pertenecerían a la mísma superficie energética, $H(\boldsymbol{I}, \boldsymbol{\theta})=h$, aunque sus energías diferirían en sólo una cantidad de $\mathcal{O}(\epsilon)$. A diferencia de lo que sucede cuando los ángulos son tales que la perturbación se anula, donde despejar alguna de las acciones en función de las restantes es una tarea directa utilizando, por ejemplo, la Ec. (4.2), cuando los ángulos son arbitrarios, como es el caso de las Ecs. (4.5)(4.6), debe adoptarse otra estrategia: $\boldsymbol{I}$ es elegida proyectando sobre la superficie isoenergética de la siguiente manera. Se despeja $I_{1}$ en función de las otras dos acciones, de los ángulos, de la

\footnotetext{
${ }^{2}$ La última condición de 4.6 es equivalente a pedir $p_{2}=p_{3}=0$.
} 
energía total y del parámetro perturbativo. Definiendo

$$
\begin{aligned}
b & \equiv \frac{\epsilon 3 \beta}{A} \mathrm{cn}^{2}\left(\frac{\theta_{1}}{\beta}, \frac{1}{\sqrt{2}}\right)\left[\mathrm{I}_{2}^{1 / 3} \operatorname{cn}\left(\frac{\theta_{2}}{\beta}, \frac{1}{\sqrt{2}}\right)+\mathrm{I}_{3}^{1 / 3} \operatorname{cn}\left(\frac{\theta_{3}}{\beta}, \frac{1}{\sqrt{2}}\right)\right], \\
c & \equiv \frac{h}{A}-\left(I_{2}^{4 / 3}+I_{3}^{4 / 3}\right),
\end{aligned}
$$

y considerando el Hamiltoniano en variables ángulo-acción dado en (3.43), la condición isoenergética se expresa así:

$$
I_{1}^{4 / 3}+b I_{1}^{2 / 3}=c .
$$

Esta ecuación tiene solución real y no negativa:

$$
\begin{aligned}
I_{1} & =\left(\frac{\sqrt{b^{2}+4 c}-b}{2}\right)^{3 / 2} \\
& \equiv H^{-1}\left(I_{2}, I_{3}, \theta_{1}, \theta_{2}, \theta_{3} ; \epsilon, h\right) .
\end{aligned}
$$

siempre que se cumpla que $\sqrt{b^{2}+4 c}-b \geq 0$.

Para el valor de $h$ utilizado en esta tesis y para el rango de valores de $\epsilon$ utilizados en las mediciones de las siguiente sección, tal inversión fue posible de realizar para todas las condiciones iniciales de $p_{1}$ y $\psi_{1}$ dadas en (4.5). Si para $\epsilon=0.02$ se realiza un nuevo mapa cromático de $\bar{Y}(3500 \times 2 \pi)$, en función de las variables canónicamente conjugadas $\psi_{1}$ y $p_{1}$, que corresponden, respectivamente, a la fase y momento resonantes, obtenemos la Fig. 4.3.

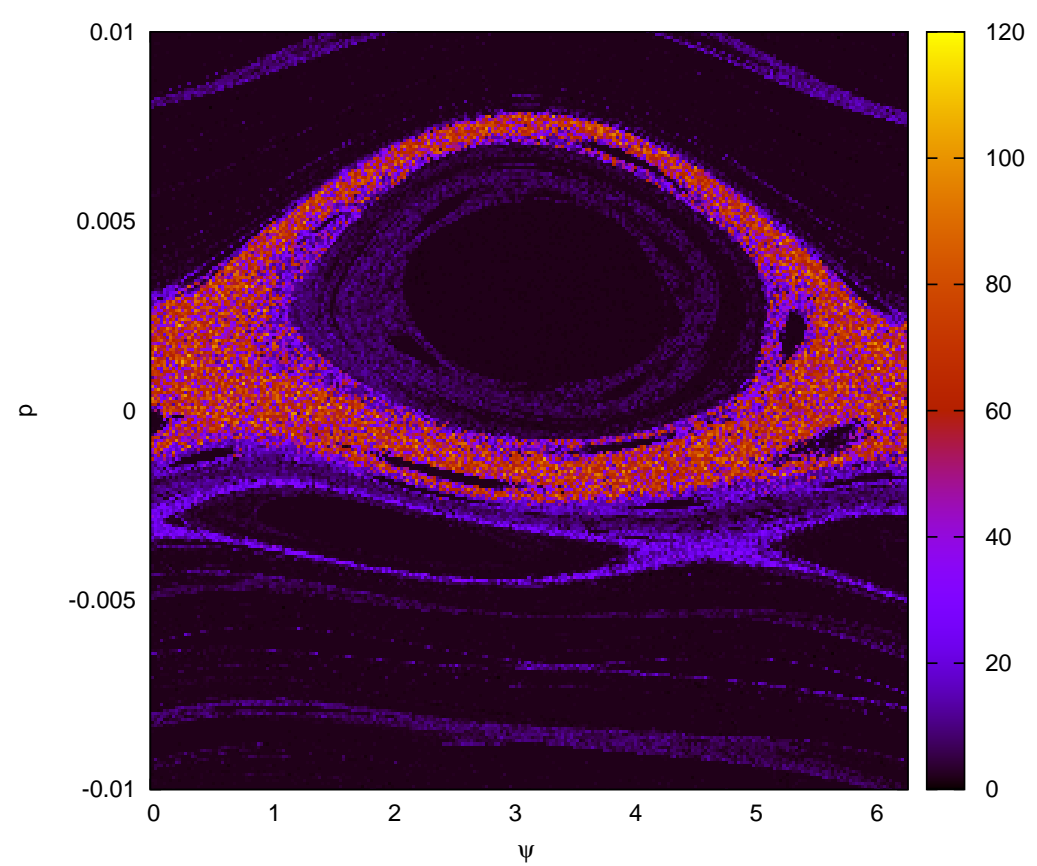

Figura 4.3: Niveles de $\bar{Y}(3500 \times 2 \pi)$ en entorno de resonancia guía, usando variables resonantes, para $\epsilon=0.02$.

El análisis del indicador nos dice que la región con colores amarillos y anaranjados es la más caótica y corresponde a la capa estocástica asociada a la resonancia. Se observa que, aunque 
estemos trabajando con un sistema en el cual existe una jerarquía infinita de resonancias que perturban a la $(2,-3,0)$, el espacio de fases resonante presenta cierta similitud con el obtenido para el sistema del péndulo simple perturbado periódicamente, como es el caso del Mapa Estandar.

Las condiciones iniciales fueron elegidas utilizando Ec. (4.6), con $\psi_{1} \in[0,0.7]$ y $p_{1} \in$ $\left[0,10^{-5}\right]$. En dicho rectángulo se estableció una grilla equiespaciada con 200 condiciones iniciales. Para los valores de $\epsilon \in \mathscr{A}_{\star}$, dichos ensambles pertenecen a las correspondientes capas estocásticas de las resonancias. En particular, se encuentran en el entorno de la fase resonante $\left(\psi_{1}, p_{1}\right)=(0,0)$, que corresponde al punto de equilibrio inestable del modelo de péndulo. En la Fig. 4.4 se señala en verde la ubicación de dicho ensamble superpuesto sobre el mapa MEGNO, para los valores de $\epsilon=0.015$ (izquierda) y $\epsilon=0.03$ (derecha).
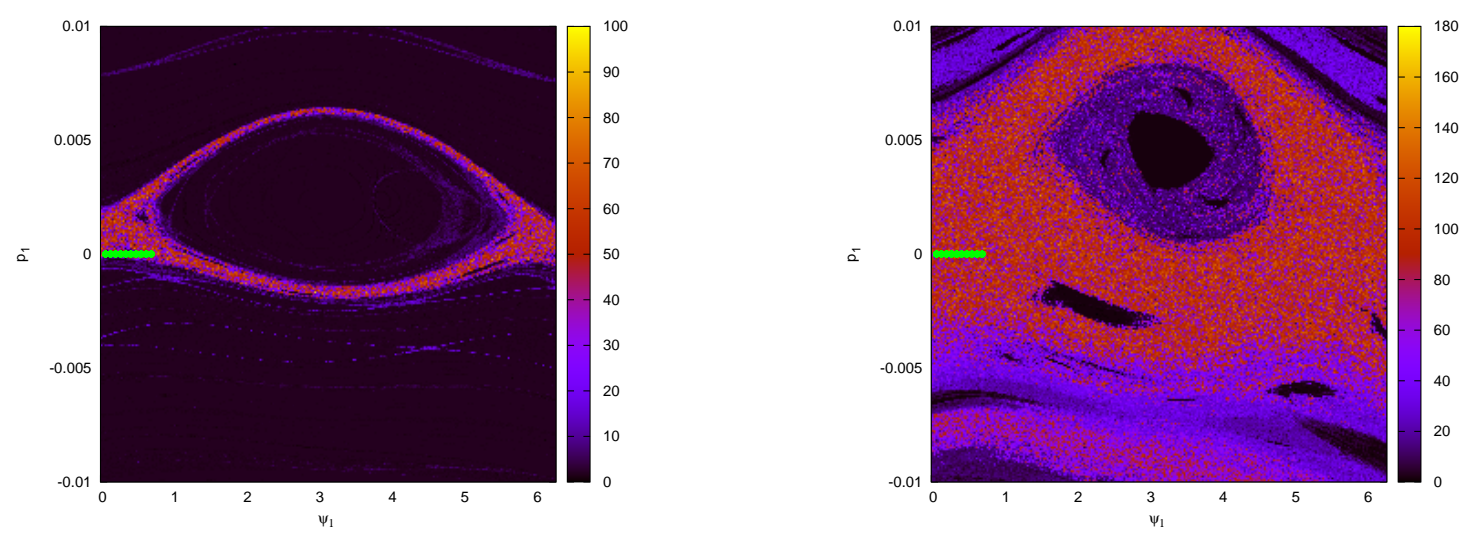

Figura 4.4: Niveles de $\bar{Y}(3500 \times 2 \pi)$ en entorno de resonancia guía, usando variables del péndulo, para $\epsilon=0.015$ (izquierda) y $\epsilon=0.030$ (derecha). Los puntos verdes identifican a las condiciones iniciales.

\subsubsection{Las mediciones}

Para los valores de $\epsilon$ pertenecientes a $\mathscr{A}_{\star}$ se integraron las trayectorias de cada uno de estos ensambles utilizando al integrador $S 8 b$ con paso de integración $\Delta t_{\text {int }}=10^{-1}$ unidades de tiempo, utilizando variables cartesianas. El paso de escritura elegido fue de tamaño $\Delta t_{e s c}=10^{2}$. La tarea del programa, antes de cada impresión, está dada por los siguientes procesos. Primero, se computan las variables acción utilizando la fórmula (3.15). Segundo, se transforman las acciones a la base de Chirikov (1.87), obteniéndose los valores del vector $\boldsymbol{p}$. Tercero, se computan las varianzas de ensamble, $\sigma_{j}^{2}(1.49)$ y desplazamientos cuadráticos medios (también de ensamble), $\gamma_{i}^{2}(1.50)$, de cada una de las variables $p_{j}(j=1,2,3)$. Dado que el vector base $\boldsymbol{\mu}_{1}$ tiene norma $\left\|\boldsymbol{m}_{g}\right\|=\sqrt{13} \neq 1$, a diferencia de los otros dos vectores base (unitarios), las cantidades estadísticas asociadas a $p_{1}$ han sido normalizadas. En otras palabras, durante el experimento $\mathscr{A}$ al utilizar la notación $\sigma_{1}, \sigma_{1}^{2}, \gamma_{1}$ y $\gamma_{1}^{2}$, nos estaremos refiriendo realmente a las cantidades $\sqrt{13} \sigma_{1}, 13 \sigma_{1}^{2}, \sqrt{13} \gamma_{1}$ y $13 \gamma_{1}^{2}$, respectivamente. Estas cantidades son las que se habrían medido si se hubiese tomado a $\boldsymbol{\mu}_{1}$ de norma uno.

Antes de analizar los resultados de la evolución de estas cantidades estadísticas se ejemplificará cualitativamente, otra vez para $\epsilon=0.02$, la dinámica de estos ensambles, median- 
te snapshots o "fotografías" de las proyecciones de los mismos sobre el plano $\left[I_{1}, I_{2}\right]$. En la Fig. 4.5-izq. se observa la situación de los ensambles para los tiempos $n \times 10^{2}$, con $n=1, \ldots, 5$, en colores rojo, verde, azul, cyan y amarillo, respectivamente. Cada punto corresponde a una partícula prueba (que aparecen superpuestas debido a su gran densidad) y la ubicación de las condiciones iniciales ha sido marcada con una cruz blanca. Debe aclararse que el hecho de que el ensamble al tiempo inicial parezca estar en la zona regular e interior a la resonancia es un efecto de proyección debido a que en este experimento los valores iniciales de los ángulos para la confección del mapa SALI son diferentes a los utilizados en las condiciones iniciales de los ensambles ${ }^{3}$. Por el mismo motivo de origen proyectivo es que para tiempos posteriores se observan partículas superpuestas sobre zonas mayoritarimante regulares.
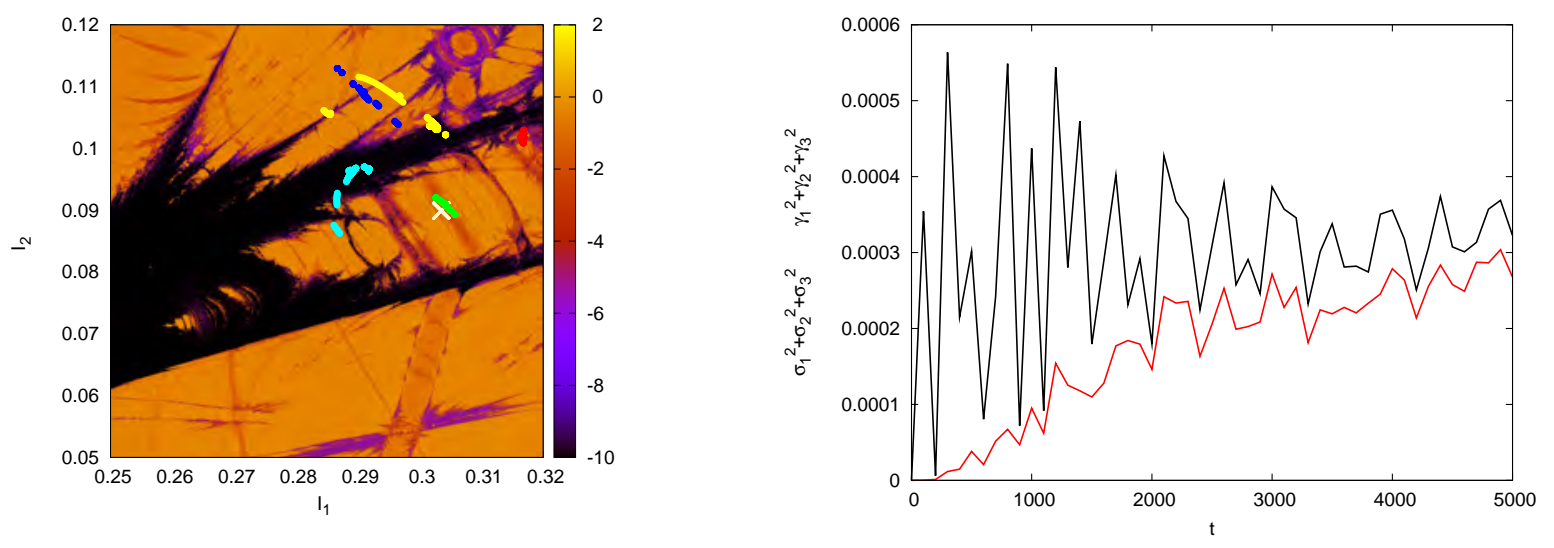

Figura 4.5: A la izquierda se muestra el ensamble para tiempos 0 (cruz blanca), $10^{2}$ (rojo), $2 \times 10^{2}$ (verde), $3 \times 10^{2}$ (azul), $4 \times 10^{2}$ (cyan), y $5 \times 10^{2}$ (amarillo). A la derecha las curvas de $\sigma_{*}^{2} \equiv \sigma_{1}^{2}+\sigma_{2}^{2}+\sigma_{3}^{2}$ (rojo) y $\gamma_{*}^{2} \equiv \gamma_{1}^{2}+\gamma_{2}^{2}+\gamma_{3}^{2}$ (negro), para $0 \leq t \leq 5 \times 10^{3}$.

Se observan principalmente dos fenómenos. Uno de ellos es un movimiento oscilatorio del centro de masa de orden de magnitud similar al del tamaño de la resonancia. El otro es una disgregación del ensamble, que está relacionada con la decorrelación entre las fases resonantes asociadas a las partículas. Estos dos fenómenos son cuantificables observando la Fig. 4.5-der. donde se muestra la evolución de las cantidades $\sigma_{*}^{2} \equiv \sigma_{1}^{2}+\sigma_{2}^{2}+\sigma_{3}^{2}$ (rojo) y $\gamma_{*}^{2} \equiv \gamma_{1}^{2}+\gamma_{2}^{2}+\gamma_{3}^{2}$ (negro), para las primeras $5 \times 10^{3}$ unidades de tiempo. El desplazamiento cuadrático medio $\left(\gamma_{*}^{2}\right)$ presenta crecimientos del orden de $3.5 \times 10^{-4}$ al menos desde las primeras 100 unidades de tiempo. Esto implica que la distancia del ensamble respecto de la condición inicial es del orden $\gamma_{*} \approx \sqrt{3.5 \times 10^{-4}} \approx 0.019$, lo cual es verificado por la ubicación de los puntos rojos del snapshot. Por otro lado, la varianza $\left(\sigma_{*}^{2}\right)$ crece lentamente y recién a partir de tiempos $\gtrsim 2000$ adquiere valores del orden de los de $\gamma_{*}^{2}$. Es a partir de este punto, que el proceso adquiere características difusivas. Esta equivalencia entre estas dos cantidades estadísticas, para tiempos mayores al mencionado transitorio, puede apreciarse en la Fig. 4.6, donde se brindan las posiciones de las partículas para tiempos de la forma $n \times 10^{m}$, con $n=1, \ldots, 5$ y $m=3, \ldots, 6$. En las mismas se puede apreciar como el ensamble se va expandiendo gradualmente. Se observa que para tiempos del orden de $10^{3}$ (Fig. 4.6-sup. izq.)

\footnotetext{
${ }^{3}$ Se recuerda que para confeccionar el mapa del indicador se inicializaron los tres ángulos en $\pi / 2$ mientras que los ensambles fueron elegidos satisfaciendo Ecs. (4.5) y (4.6).
} 

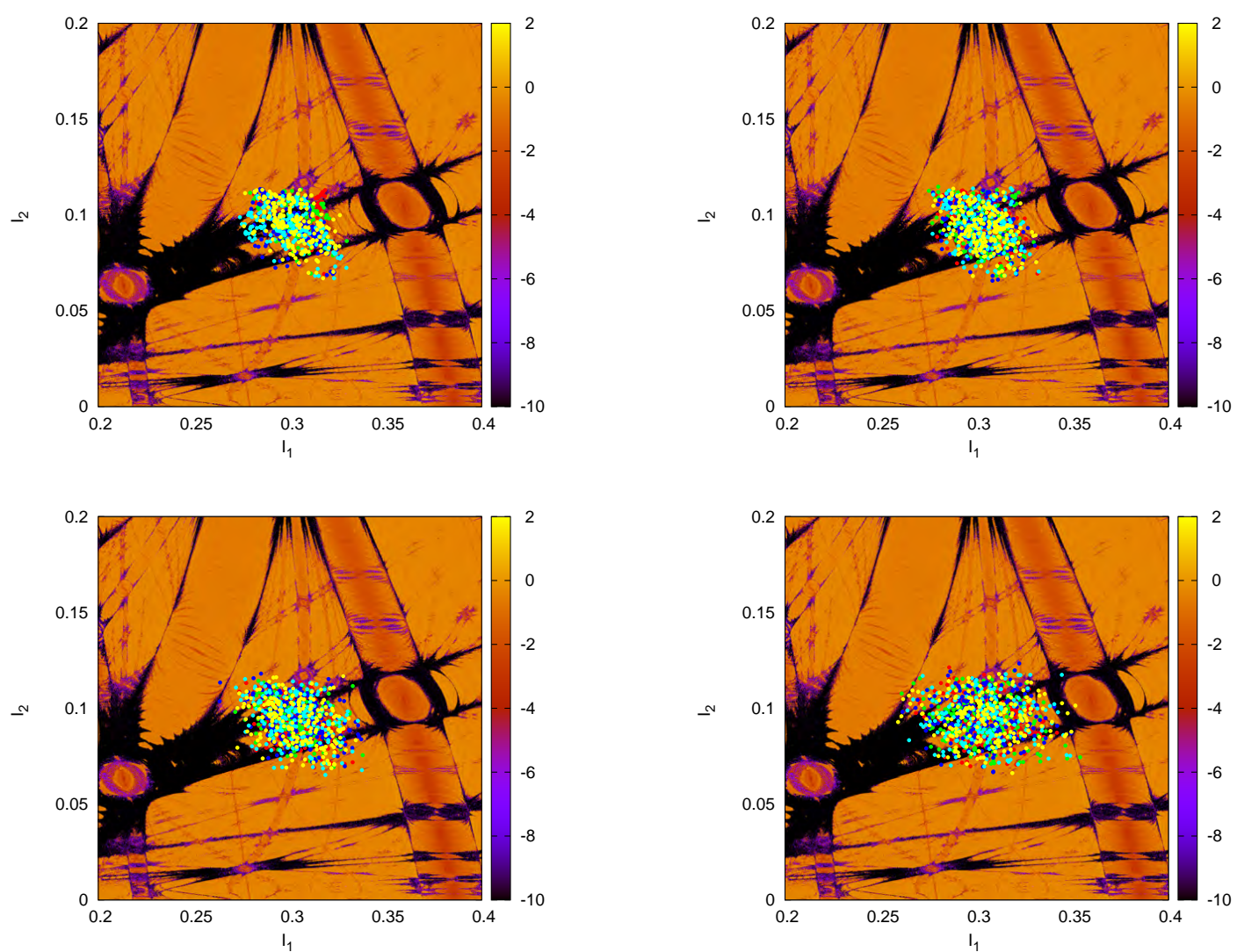

Figura 4.6: La imagen sup. izq. corresponde a tiempos $10^{3}$ (rojo), $2 \times 10^{3}$ (verde), $3 \times 10^{3}$ (azul), $4 \times 10^{3}$ (cyan) y $5 \times 10^{3}$ (amarillo). La imagen sup. der. corresponde a tiempos $10^{4}$ (rojo), $2 \times 10^{4}$ (verde), $3 \times 10^{4}$ (azul), $4 \times 10^{4}$ (cyan) y $5 \times 10^{4}$ (amarillo). La imagen inf. izq. corresponde a tiempos $10^{5}$ (rojo), $2 \times 10^{5}$ (verde), $3 \times 10^{5}$ (azul), $4 \times 10^{5}$ (cyan) y $5 \times 10^{5}$ (amarillo). La imagen inf. der. corresponde a tiempos $10^{6}$ (rojo), $2 \times 10^{6}$ (verde), $3 \times 10^{6}$ (azul) y $4 \times 10^{6}$ (cyan) y $5 \times 10^{6}$ (amarillo).

la extensión del ensamble en dirección paralela a $\boldsymbol{\mu}_{1}$ es levemente mayor a aquella en dirección paralela a $\boldsymbol{\mu}_{3}$. Sin embargo, tal situación se invierte al considerar tiempos del orden de $10^{6}$ (Figs. 4.6-inf. der. y 4.7-izq.), en donde se evidencia la existencia de transporte macroscópico neto a lo largo de la resonancia guía.

En la Fig. 4.7-izq., que muestra los ensambles para tiempos $n \times 10^{6}$, con $n=6, \ldots, 10$, en colores rojo, verde, azul, cyan y amarillo, se demuestra que hay partículas que arriban a los entornos de las resonancias vecinas $(2,-1,-1)$ y $(2,0,-2)$. Las curvas de $\sigma_{*}^{2}(t)$ y $\gamma_{*}^{2}(t)$ se despliegan en la Fig. 4.7-der. para $0 \leq t \leq 10^{7}$. Con el objeto de conocer la dependencia funcional de la varianza con el tiempo, se asumió como ansatz una ley de potencias de la forma

$$
\sigma_{*}^{2}=\kappa t^{w},
$$

que es equivalente a la correspondiente dependencia de un movimiento Browniano fraccional (1.74), si se asocia $w$ con el exponente de Hurst según $w=2 v$. Aplicando el logaritmo 

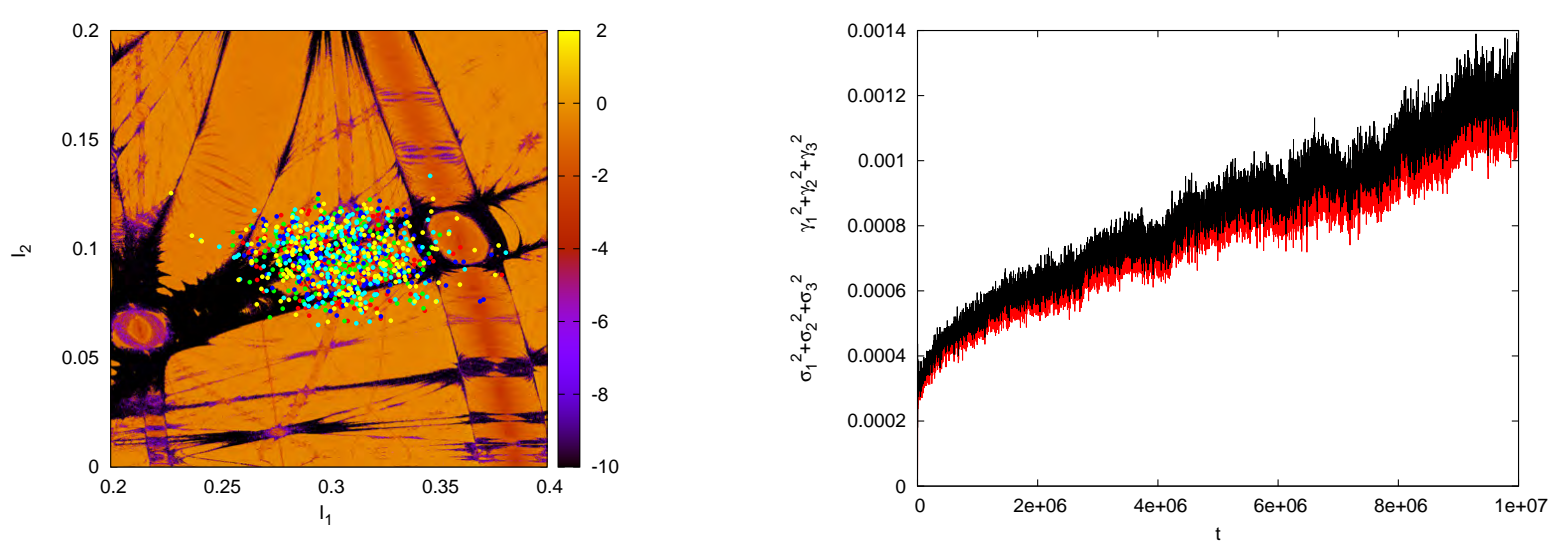

Figura 4.7: A la izquierda se muestra el ensamble para tiempos de la forma $n \times 10^{6}$, con $n=6, \ldots, 10$, en colores rojo, verde, azul, cyan y amarillo, respectivamente. A la derecha las curvas de $\sigma_{*}^{2} \equiv \sigma_{1}^{2}+\sigma_{2}^{2}+\sigma_{3}^{2}$ (rojo) y $\gamma_{*}^{2} \equiv \gamma_{1}^{2}+\gamma_{2}^{2}+\gamma_{3}^{2}$ (negro), para $0 \leq t \leq 10^{7}$.

decimal a ambos miembros de (4.10), se obtiene

$$
\log \left(\sigma_{*}^{2}\right)=\log (\kappa)+w \log (t)
$$

lo cual implica una relación lineal entre el logaritmo del tiempo y el logaritmo de la varianza, siendo la pendiente de la misma $(w)$ el exponente de la mencionada ley de potencias. Un ajuste numérico de las variables $\log (\kappa)$ y $w$, para $t \in\left[10^{5}, 10^{6.5}\right]$, dió como resultado que $\kappa \approx 2.23 \times 10^{-5}$ y que $w \approx 0.225$. El hecho de que $w$ resultó ser considerablemente menor a la unidad, es una prueba directa de que durante ese intervalo de tiempo la difusión es anómala, y en particular, es un proceso subdifusivo. La longitud de este intervalo de tiempo, que abarca alrededor de $3 \times 10^{6}$ unidades de tiempo, no es despreciable. Por el contrario, observando los partículas en azul de la Fig. 4.6-inf. der, se tiene que este intervalo conforma gran parte de la etapa en que el ensamble está exclusivamente contenido en la resonancia guía, antes de que las partículas lleguen a los cruces con otras resonancias. La Fig. 4.8-izq. muestra a $\sigma_{*}^{2}(t)$ en escala logarítmica, junto con el ajuste. Por otro lado, la Fig. 4.8-der. evidencia que dicho ajuste se aparta de la medición real para tiempos mayores.

A continuación consideramos la evolución de las varianzas individuales asociadas a cada dirección. La Fig. (4.9) despliega las curvas $\sigma_{1}^{2}(t), \sigma_{2}^{2}(t)$ y $\sigma_{3}^{2}(t)$ respectivamente en colores rojo, verde y azul, para el mismo intervalo de tiempo anteriormente utilizado. Se observa una aproximada estabilización de $\sigma_{1}^{2}$ y $\sigma_{2}^{2}$ en valores constantes, al tiempo que $\sigma_{3}^{2}$ posee un crecimiento neto con el tiempo. En el caso de $\sigma_{2}^{2}$, dicha estabilización se debe a la conservación de la energía (1.76). En el caso de $\sigma_{1}^{2}$ se debe a que el momento resonante $\left(p_{1}\right)$ está acotado debido a la conservación (local y aproximada) de la energía de la forma normal del péndulo. Esta es la situación típica que se esperaría en un proceso de difusión de Arnold (secciones 1.4.2 y 1.4.4), durante la etapa en que el ensamble está mayoritariamente contenido en la capa estocástica de la resonancia guía inicial. Una vez que la difusión procede considerablemente a lo largo de otras resonancias, la base de Chirikov asociada a la resonancia guía original deja de tener sentido geométrico. Cuando se cambia de resonancia guía, no sólo cambian los vectores $\boldsymbol{\mu}_{1}$ y $\boldsymbol{\mu}_{3}$ sino que, debido a la convexidad de $\mathcal{I}_{0}$, también lo hace $\boldsymbol{\mu}_{2}$. 

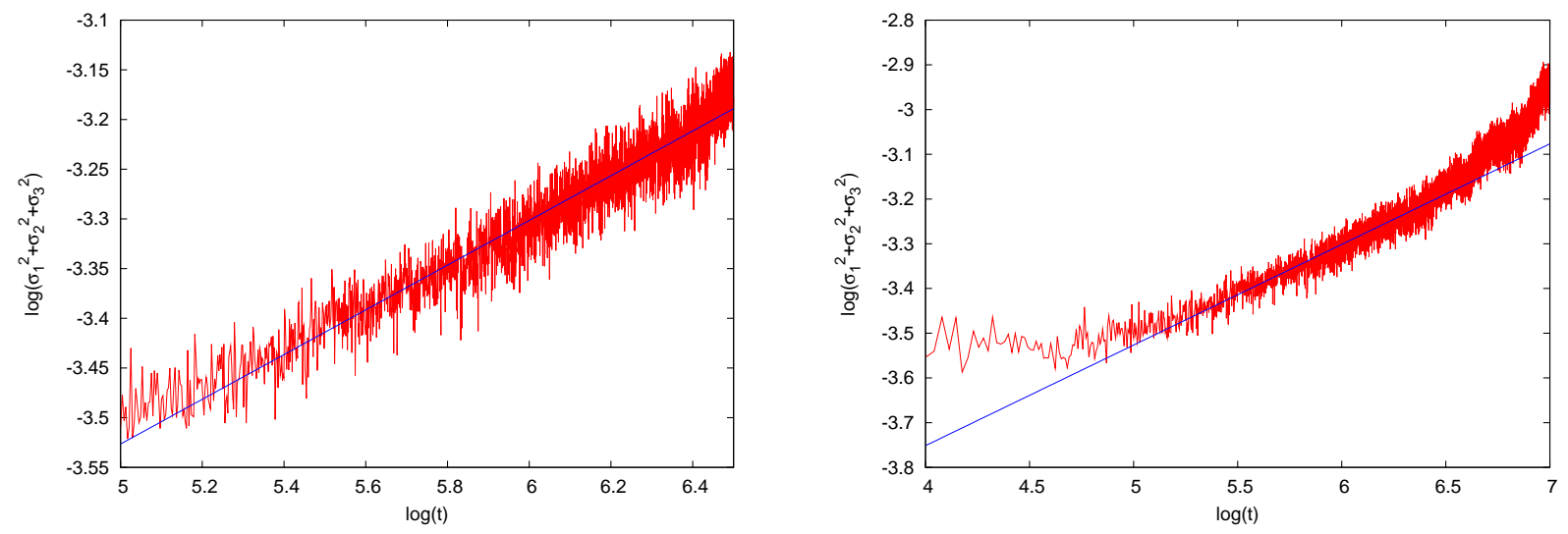

Figura 4.8: A la izquierda se muestra $\log \left(\sigma_{*}^{2}\right)$ en función $\log (t)$ (rojo) junto con el ajuste lineal de pendiente $w \approx 0.225$ (azul), para $t \in\left[10^{5}, 10^{6.5}\right]$. A la derecha se comprueba que este comportamiento tipo ley de potencias $\left(\sigma_{*}^{2} \propto t^{w}\right)$ deja de ser válida al considerar un intervalo de tiempo más ámplio.

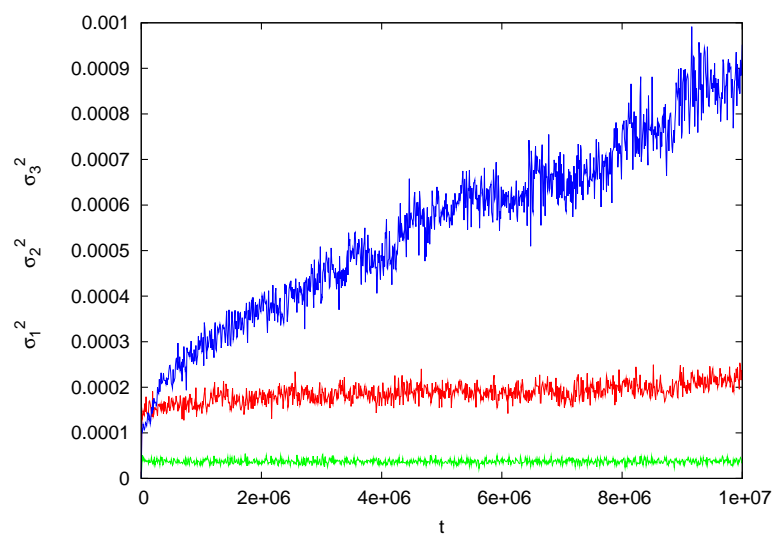

Figura 4.9: $\sigma_{1}^{2}(t), \sigma_{2}^{2}(t)$ y $\sigma_{3}^{2}(t)$ respectivamente en colores rojo, verde y azul, para $\epsilon=0.02 \mathrm{y}$ $h$ canónico.

En la Fig. 4.10 se despliegan los valores de las tres varianzas para cada uno de los valores pertencientes a $\mathscr{A}_{\star}$. Se evidencian cambios en el comportamiento de las varianzas a medida que se aumenta gradualmente la intensidad de la perturbación. De las Figs. 4.10 (a,b,c) se observa que para $\epsilon \in\{0.016,0.018\}$ el comportamiento es cualitativamente similar al obtenido para $\epsilon=0.020$. Las Figs. 4.10 (d,e,f) muestran que para $\epsilon \in\{0.022,0.024,0.026\}, \sigma_{1}^{2}$ no se estabiliza en un valor constante, sino que posee un crecimiento neto. Por otro lado, $\sigma_{2}^{2}$ sí converge aproximadamente a un valor constante, mientras que $\sigma_{3}^{2}$ sigue siendo la varianza que más crece en el intervalo de temporal analizado. Por último, en las Figs 4.10 (g,h) se nota que para $\epsilon \in\{0.028,0.030\}, \sigma_{2}^{2}$ presenta un crecimiento neto y, además, la magnitud del crecimiento de $\sigma_{1}^{2}$ es del orden de $\sigma_{3}^{2}$. El hecho de que para $\epsilon$ tan chico como 0.028 exista un comportamiento que claramente no corresponde con una difusión de Arnold, no esta en contradicción con el resultado obtenido en capítulo anterior, en el que se determinó que 


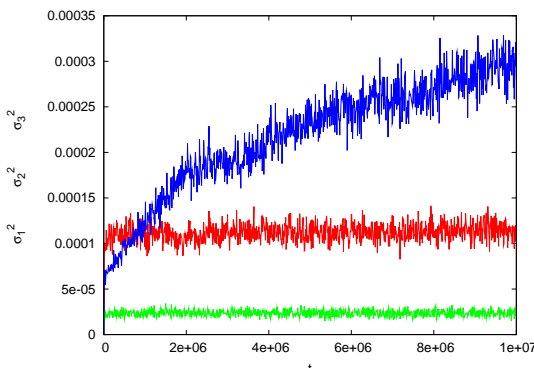

(a) $\epsilon=0.016$

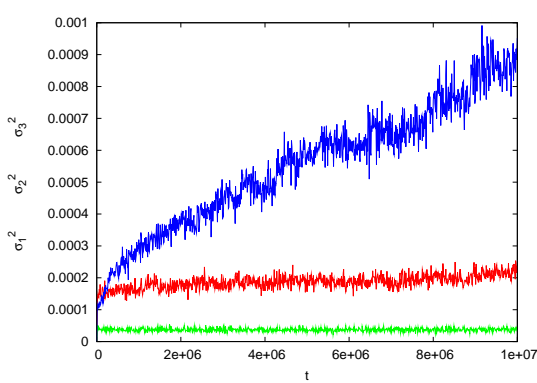

(c) $\epsilon=0.020$

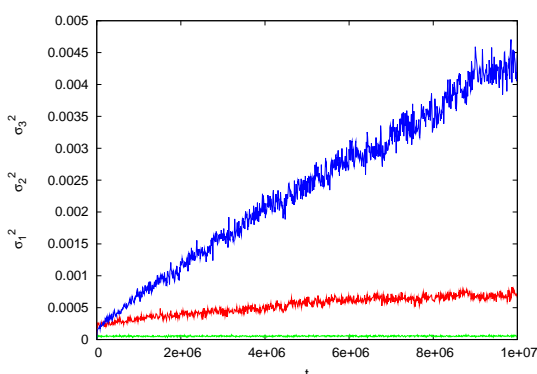

(e) $\epsilon=0.024$

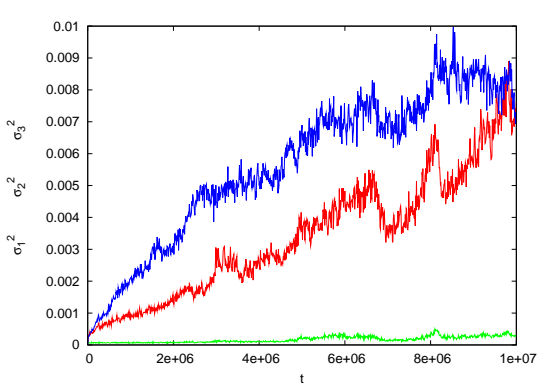

(g) $\epsilon=0.028$

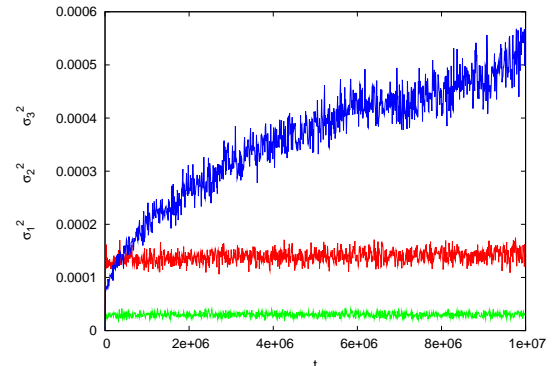

(b) $\epsilon=0.018$

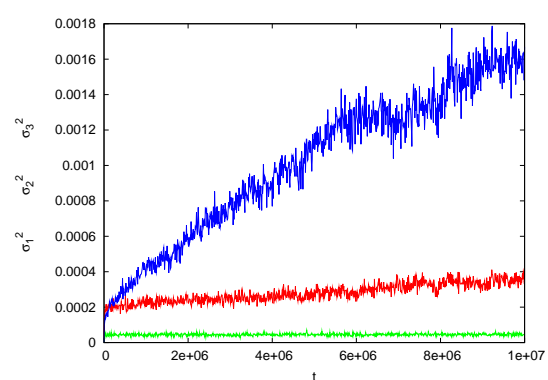

(d) $\epsilon=0.022$

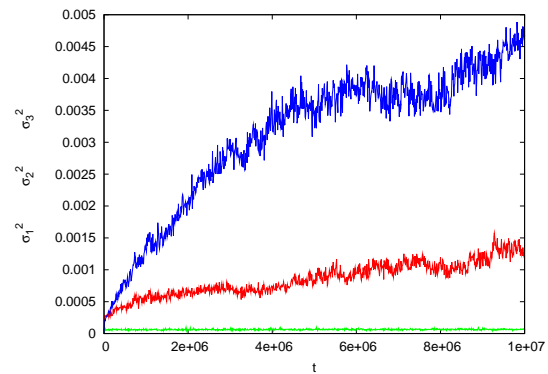

(f) $\epsilon=0.026$

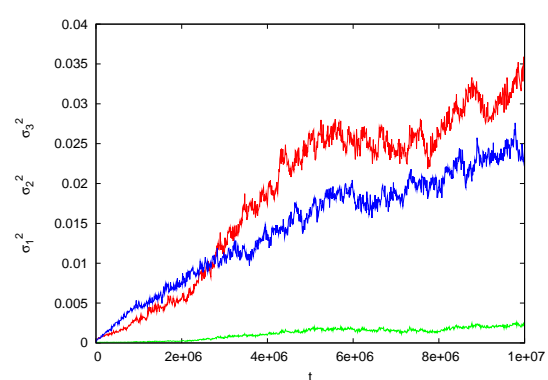

(h) $\epsilon=0.030$

Figura 4.10: $\sigma_{i}^{2}(t)(i=1,2,3)$ respectivamente en colores rojo, verde y azul, para $\epsilon \in \mathscr{A}_{\star}$ y $h$ canónico.

$\epsilon_{c}>0.030$. La explicación de ello radica en que el valor crítico es una cantidad que considera globalmente el grado de solapamiento entre resonancias, mientras que si se considera sólo el 
entorno local de la resonancia guía bajo estudio, y de la acción resonante particular el overlap se manifiesta para valores más pequeños de la perturbación. Por ejemplo, la Fig. 4.4-der., muestra que para $\epsilon=0.030$, en el entorno de este valor particular de $\boldsymbol{I}^{r}$, la resonancia se halla en un avanzado régimen de overlap.

Una vez que hemos analizado como son los cambios de las varianzas (asociadas a las tres direcciones de la mencionada base) que tienen lugar al pasar desde un régimen de Nekhoroshev hacia un régimen de Chirikov, se realizará un estudio cuantitativo trabajando sólo con $\sigma_{3}^{2}$. En la Fig. 4.11-izq. se graficó $\sigma_{3}^{2}(t)$, en escala logarítmica, para $\epsilon \in \mathscr{A}_{\star}$, en colores rojo, verde, azul, magenta, cyan, amarillo, negro y naranja, respectivamente. Se observa que, en general, para cada tiempo fijo $t$, a mayor valor de $\epsilon$, mayor es el valor de $\sigma_{3}^{2}(t)$. Para cada uno de estas curvas se realizó un ajuste lineal de forma similar a lo realizado anteriormente para la cantidad $\sigma_{*}^{2}$, utilizando el mismo intervalo temporal: $t \in\left[10^{5}, 10^{6.5}\right]$. En la Tabla 4.1 se muestran los valores de los ajustes. En la quinta columna se agregó la cantidad $w_{*}$, que corresponde con el valor del exponente ajustado para un intervalo temporal más amplio, $t \in\left[10^{5}, 10^{7}\right]$, mientras que en la sexta columna se ha agregado la diferencia relativa porcentual correspondiente. Puede apreciarse que para algunos valores de $\epsilon$, dicha diferencia llega a valores mayores al $15 \%$, lo cual es una prueba más de que los comportamientos de las cantidades estadísticas son altamente dependientes del intervalo temporal analizado. En la Fig. 4.11-der. se muestra gráficamente la concordancia entre los ajustes lineales realizados en $10^{5} \leq t \leq 10^{6.5}$ y las correspondientes curvas. Análogamente, en la Fig. 4.12-izq., se despliega la situación obtenida al considerar el rango $10^{5} \leq t \leq 10^{7}$. En la Fig. 4.12-der. se muestran los valores de los exponents $w$ y $w_{*}$ en función de $\epsilon$, respectivamente en colores rojo y negro.
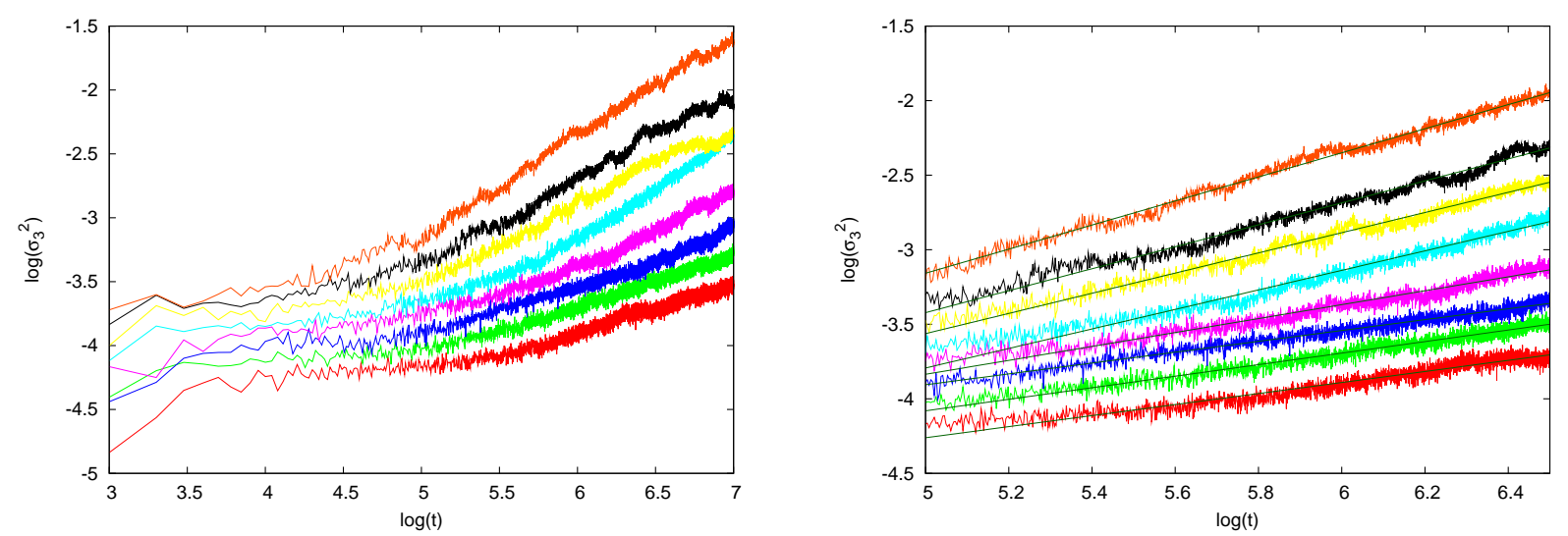

Figura 4.11: $\sigma_{3}^{2}(t)$ en escala logarítmica, para $\epsilon \in \mathscr{A}_{\star}$, respectivamente en colores rojo, verde, azul, magenta, cyan, amarillo, negro y naranja. En la figura de la izquierda se utilizó $t \in$ $\left[10^{3}, 10^{7}\right]$. En la figura de la derecha se han agregado las correspondientes rectas que ajustan aproximadamente a dichas curvas, para $t \in\left[10^{5}, 10^{6.5}\right]$.

Debido a que todos los valores de $w\left(\mathrm{y}\right.$ de $w_{*}$ ) son inferiores a la unidad, se puede concluir que para los dos rangos temporales y para los ocho valores de $\epsilon$ recién analizados, la evolución de las varianzas corresponden a procesos de difusión anómala del tipo subdifusiva.

A pesar de esto, la Fig. 4.13 indica que al considerar tiempos suficientemente menores a los recientemente utilizados, las varianzas siguen un comportamiento aproximadamente lineal. 


\begin{tabular}{|c|c|c|c|c|c|}
\hline$\epsilon$ & $w$ & $\log (\kappa)$ & $\kappa$ & $w_{*}$ & $\frac{\mid w-w_{*}}{w} \times 100$ \\
\hline 0.016 & 0.370366 & -6.11277 & $7.713 \times 10^{-7}$ & 0.365061 & $\sim 1$ \\
0.018 & 0.387073 & -6.01603 & $9.638 \times 10^{-7}$ & 0.395128 & $\sim 2$ \\
0.020 & 0.363961 & -5.72554 & $1.881 \times 10^{-6}$ & 0.444984 & $\sim 22$ \\
0.022 & 0.466993 & -6.17054 & $6.752 \times 10^{-7}$ & 0.564104 & $\sim 20$ \\
0.024 & 0.654695 & -7.06677 & $8.575 \times 10^{-8}$ & 0.755714 & $\sim 15$ \\
0.026 & 0.676915 & -6.94744 & $1.129 \times 10^{-7}$ & 0.562896 & $\sim 16$ \\
0.028 & 0.736347 & -7.10383 & $7.874 \times 10^{-8}$ & 0.655036 & $\sim 11$ \\
0.030 & 0.807722 & -7.19636 & $6.363 \times 10^{-8}$ & 0.756279 & $\sim 6$ \\
\hline
\end{tabular}

Tabla 4.1: Resultados del ajuste numérico, de acuerdo a ansatz similar al dado por (4.10), con la correspondiente substitución de $\sigma_{*}$ por $\sigma_{3}$. En la quinta columna se agregó la cantidad $w_{*}$, que corresponde al valor del exponente ajustado para un intervalo temporal más amplio, $t \in\left[10^{5}, 10^{7}\right]$, mientras que en la sexta columna se ha agregado la diferencia relativa porcentual correspondiente.
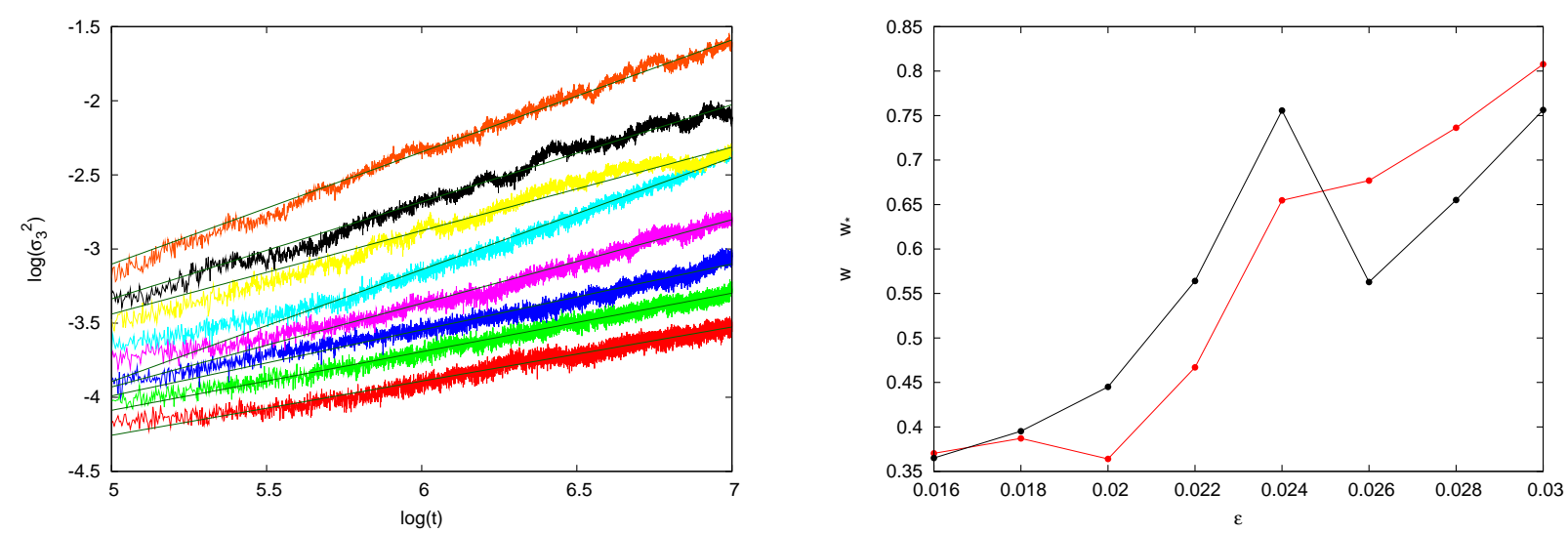

Figura 4.12: En la figura izquierda se despliegan las curvas de $\sigma_{3}^{2}(t)$ en escala logarítmica, para $\epsilon \in \mathscr{A}$, respectivamente en colores rojo, verde, azul, magenta, cyan, amarillo, negro y naranja, junto con las correspondientes rectas que ajustan aproximadamente a dichas curvas, para $t \in\left[10^{5}, 10^{7}\right]$. La figura derecha muestra las curvas $w(\epsilon)$ y $w_{*}(\epsilon)$, respectivamente en colores rojo y negro.

Por lo tanto, a modo de tener una estimación de la tasa de cambio promedio de la varianza durante los primeros $5 \times 10^{5}$ unidades de tiempo, se computará el coeficiente de difusión $\mathcal{D}_{\sigma}$ (1.40), eligiendo como tiempo inicial $t_{0}=10^{4}$ y como tiempo final $t=5 \times 10^{5}$. Dicha información es brindada por la Tabla 4.2 y por la Figura 4.14, en función de $\epsilon$. Observando esta figura no es posible determinar la relación funcional entre el coeficiente de difusión y el parámetro perturbativo. Debido a la pequeñez del rango utilizado en $\epsilon$, los datos obtenidos podrían ser compatibles con varios tipos de formas funcionales, como por ejemplo una ley de potencias o una ley exponencial. En el experimento de la próxima sección se analizará un conjunto de valores de $\epsilon$ que abarca un rango de tamaño mayor. 

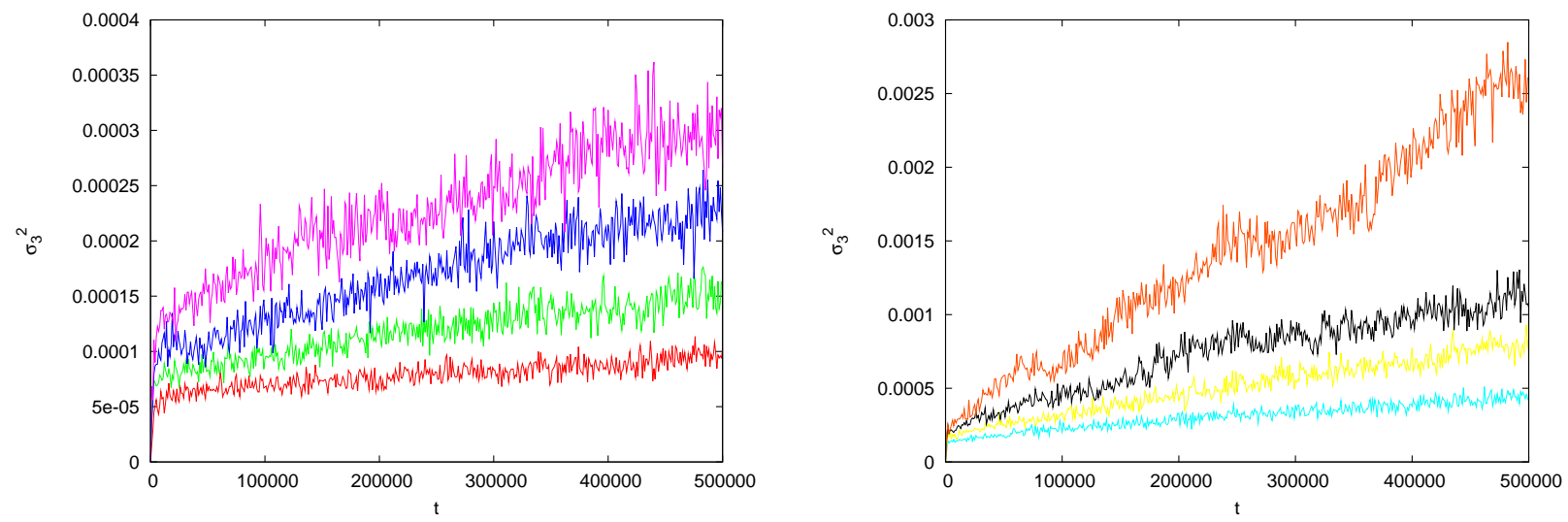

Figura 4.13: $\sigma_{3}^{2}(t)$ para $\epsilon \in \mathscr{A}_{\star}$, para $t \leq 5 \times 10^{5}$. Cada color identifica a un valor de $\epsilon$, siguiendo el mismo criterio utilizado en la Fig. 4.11.

\begin{tabular}{|c|c|c|c|}
\hline$\epsilon$ & $\sigma_{3}^{2}\left(t_{0}\right)$ & $\sigma_{3}^{2}(t)$ & $\mathcal{D}_{\sigma}$ \\
\hline 0.016 & $0.571 \times 10^{-4}$ & $0.102 \times 10^{-3}$ & $4.59 \times 10^{-11}$ \\
0.018 & $0.739 \times 10^{-4}$ & $0.162 \times 10^{-3}$ & $9.01 \times 10^{-11}$ \\
0.020 & $0.107 \times 10^{-3}$ & $0.208 \times 10^{-3}$ & $1.02 \times 10^{-10}$ \\
0.022 & $0.136 \times 10^{-3}$ & $0.265 \times 10^{-3}$ & $1.31 \times 10^{-10}$ \\
0.024 & $0.140 \times 10^{-3}$ & $0.414 \times 10^{-3}$ & $2.78 \times 10^{-10}$ \\
0.026 & $0.153 \times 10^{-3}$ & $0.839 \times 10^{-3}$ & $6.99 \times 10^{-10}$ \\
0.028 & $0.237 \times 10^{-3}$ & $0.105 \times 10^{-2}$ & $8.30 \times 10^{-10}$ \\
0.030 & $0.288 \times 10^{-3}$ & $0.261 \times 10^{-2}$ & $2.37 \times 10^{-9}$ \\
\hline
\end{tabular}

Tabla 4.2: $\mathcal{D}_{\sigma}(\epsilon)$, utilizando $t_{0}=10^{4}$ y $t=5 \times 10^{5}$.

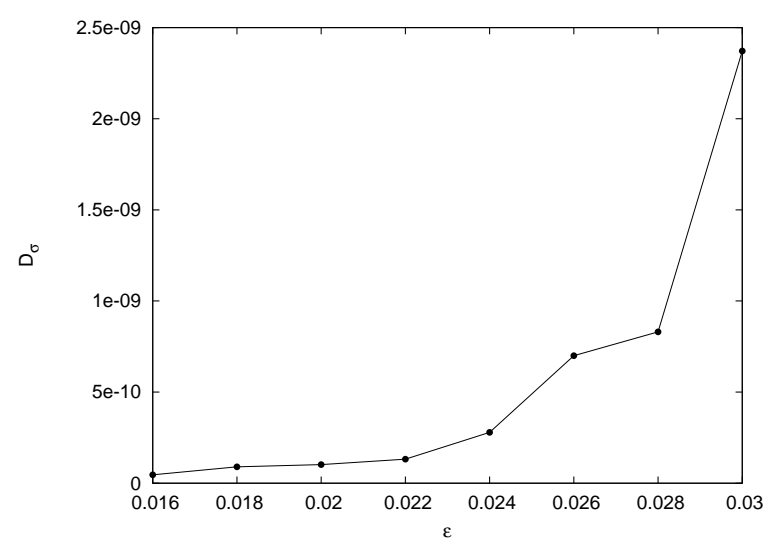

Figura 4.14: $\mathcal{D}_{\sigma}(\epsilon)$, utilizando $t_{0}=10^{4}$ y $t=5 \times 10^{5}$.

\subsection{Experimento $\mathscr{B}$}

En este experimento sólo nos interesaremos por el comportamiento de la cantidad estadística $\sigma_{3}^{2}$. Se considerará un conjunto de valores de $\epsilon$ que abarca casi tres órdenes de magnitud 
dado por:

$$
\begin{aligned}
\mathscr{B}_{\star}=\left\{4 \times 10^{-3}, 6 \times 10^{-3}, 8 \times 10^{-3}, 10^{-2}, 2 \times 10^{-2},\right. \\
\\
\left.4 \times 10^{-2}, 6 \times 10^{-2}, 8 \times 10^{-2}, 10^{-1}\right\} .
\end{aligned}
$$

\subsubsection{Los ensambles}

La elección de las condiciones iniciales se hará de forma diferente a lo realizado en los cálculos de difusión del experimento anterior, ya que ahora las mismas serán elegidas aleatoriamente tomando entornos sobre el plano $\left[I_{1}, I_{2}\right]$ y fijando todos los ángulos en $\pi / 2$. Dichos entornos serán cuadrados de área $4 \times 10^{-12}$. En la Figura 4.15 se puede apreciar el mapa SALI correspondiente a cada uno de los mencionados valores de $\mathscr{B}_{\star}$, para una grilla de condiciones iniciales ubicadas en el cuadrado $\left(I_{1}, I_{2}\right) \in[0.25,0.32] \times[0.05,0.12]$. Se observan los cambios que suceden en la estructura resonante a medida que aumenta $\epsilon$. Se puede apreciar como se van ensanchando las resonancias y las capas estocásticas al tiempo que se produce la transición entre un régimen de Nekhoroshev y uno de Chirikov. Al igual que en la experiencia $\mathscr{A}$, se trabajará con una acción resonante fija, que en este caso es la siguiente:

$$
\boldsymbol{I}^{r}=\left(\begin{array}{l}
0.290000000000000 \\
0.085925925925926 \\
0.434838361446344
\end{array}\right)
$$

La misma se halla muy cercana a la que fue utilizada en la experiencia anterior, dada por Ec. (4.4). Este ligero cambio en la elección de la acción resonante, es al efecto de experimentar, un poco, la sensibilidad de los resultados de difusión.

La proyección de dicha acción resonante sobre el plano $\left[I_{1}, I_{2}\right]$ ha sido señalada con un punto rojo en las mencionadas figuras. A su vez, se ha estimado teóricamente la ubicación de la separatriz (rama superior) correspondiente a esta acción resonante, mediante la utilización de la fórmula:

$$
\boldsymbol{I}=\boldsymbol{I}^{r}+p_{s}\left(\psi_{s}(0)\right) \boldsymbol{m}_{g},
$$

donde la función $p_{s}\left(\psi_{s}\right)$ está dada por la Ec. (3.28) y donde $\psi_{s}(0)=-\pi / 2$ es el valor que corresponde a los ángulos iniciales elegidos para esta resonancia guía, teniendo en cuenta la argumentación brindada al final de la sección 3.9. Las ubicaciones de estas acciones han sido señaladas con puntos verdes en sus correspondientes separatrices. Dado que la posición de la separatriz teórica de una resonancia, puede presentar diferencias respecto de la posición de la verdadera capa estocástica, no resulta adecuado utilizar estos puntos verdes como centros de las condiciones iniciales de los ensambles con los cuales se pretende medir la difusión; en especial al trabajar con perturbaciones pequeñas, donde las capas estocásticas son muy delgadas. No obstante, estos puntos verdes fueron utilizados como centros de nuevos mapas SALI, con el propósito de amplificar dichos entornos, que son desplegados en la Fig. 4.16. Para asegurar que los ensambles elegidos estén totalmente contenidos en la capa estocástica, se realizó otro proceso de amplificación para los cuatro valores de $\epsilon$ de menor tamaño pero estas imágenes no se muestran por no aportar nueva información. Los centros de dichos ensambles, se detallan en la Tabla 4.3 y han sido señalados con cruces blancas en las correspondientes imágenes de la Fig. 4.16. La precisión en el cómputo de cantidades estadísticas fue mejorada respecto a los experimentos $\mathscr{A}$, ya que aquí se utilizan ensambles de $N_{p}=10^{3}$ partículas. 


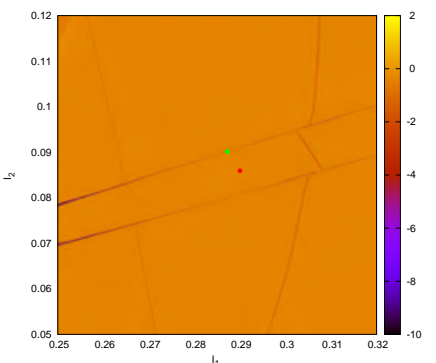

(a) $\epsilon=4 \times 10^{-3}$

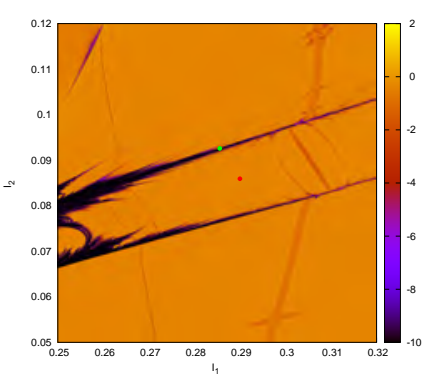

(d) $\epsilon=10^{-2}$

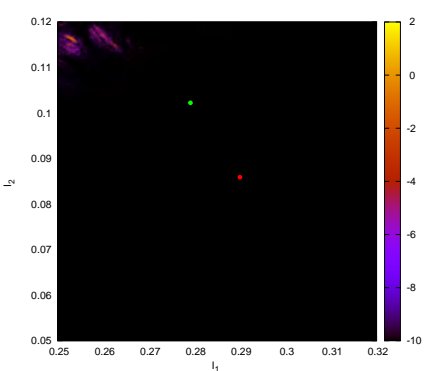

(g) $\epsilon=6 \times 10^{-2}$

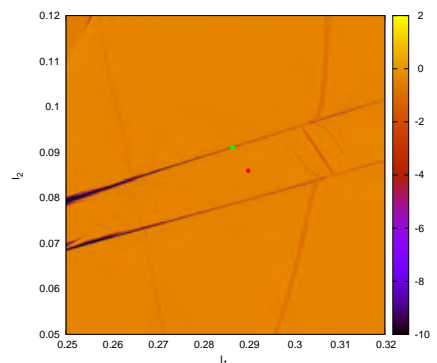

(b) $\epsilon=6 \times 10^{-3}$

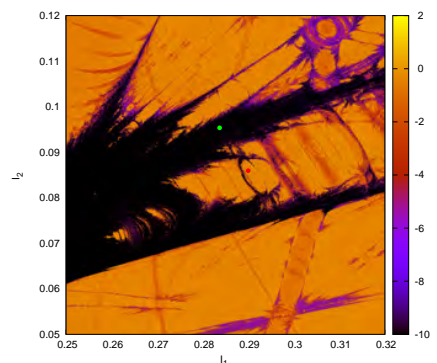

(e) $\epsilon=2 \times 10^{-2}$

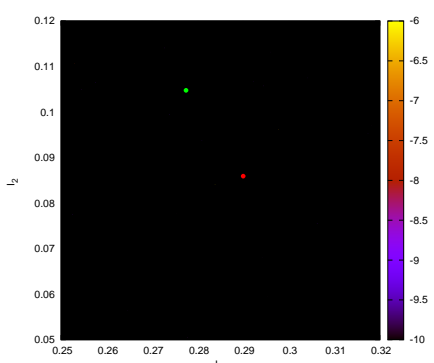

(h) $\epsilon=8 \times 10^{-2}$

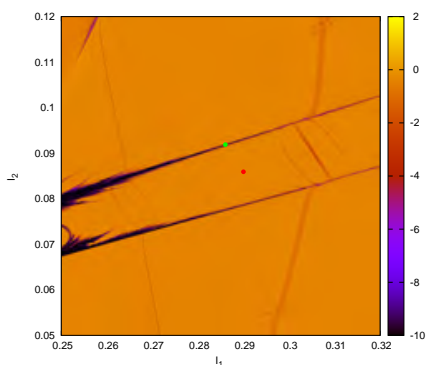

(c) $\epsilon=8 \times 10^{-3}$

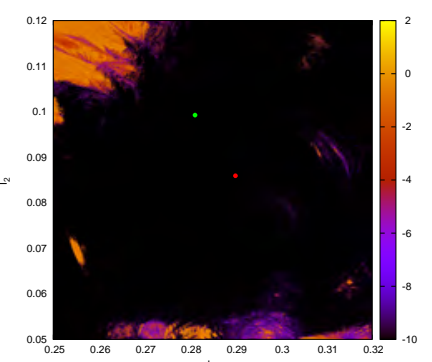

(f) $\epsilon=4 \times 10^{-2}$

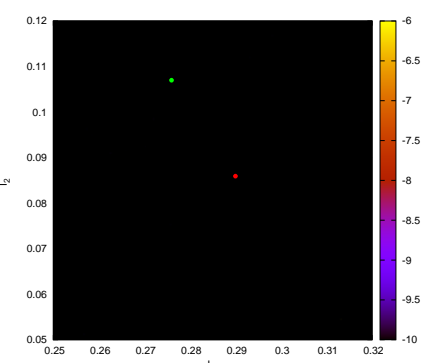

(i) $\epsilon=10^{-1}$

Figura 4.15: Estructura resonante para $\epsilon \in \mathscr{B}_{\star}$ y $h$ canónico. Los punto rojos señalan la acción resonante utilizada en los experimentos de esta sección. Los puntos verdes son la estimación teórica de la posición de la separatriz correspondiente a dicha acción resonante.

\subsubsection{Las mediciones}

En esta primera etapa del experimento se trabajó con el integrador $S 8 b$ utilizando un paso de integración $\Delta t_{i n t}=10^{-2}$ y alcanzando un tiempo total de $10^{6.5}$. El paso de escritura fue $\Delta_{\text {esc }}=10^{3}$. Para todos los ensambles se cumple que $\sigma_{3}^{2}(0) \approx 0.7 \times 10^{-12}$. En la Fig. 4.17 se muestra en escala logarítmica la evolución de $\sigma_{3}^{2}(t)$ para $\epsilon \in \mathscr{B}_{\star}$ y $t \in\left[10^{3}, 10^{6.5}\right]$. De la misma se puede concluir que en el rango de intensidades de la perturbación consideradas existen comportamientos muy disímiles entre sí. Por un lado, para $\epsilon$ igual a $4 \times 10^{-3}$ (rojo), $6 \times 10^{-3}$ (verde), $8 \times 10^{-3}$ (azul), $10^{-2}$ (magenta) y $2 \times 10^{-2}$ (cyan) se observa que para tiempos del orden de $10^{3}$, las correspondientes varianzas han alcanzado valores superiores a $10^{-6}$. Este comportamiento inicialmente abrupto es similar a lo observado en la experiencia de 


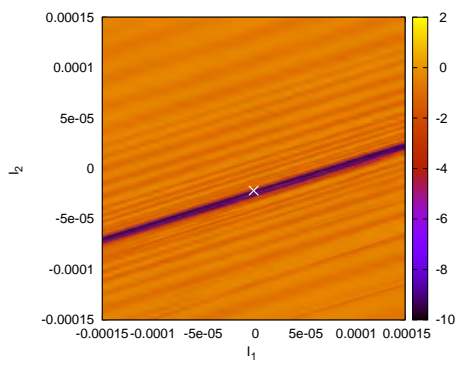

(a) $\epsilon=4 \times 10^{-3}$

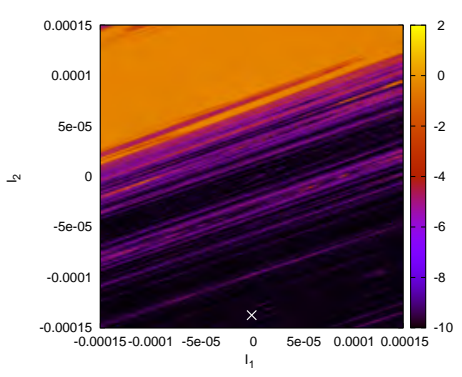

(d) $\epsilon=10^{-2}$

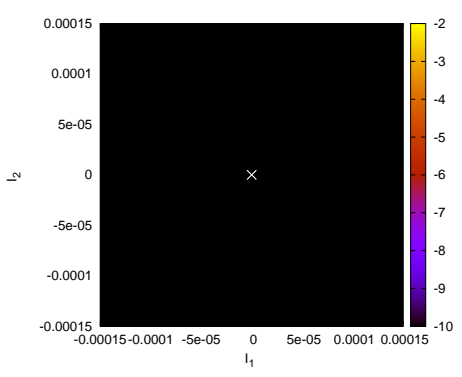

(g) $\epsilon=6 \times 10^{-2}$

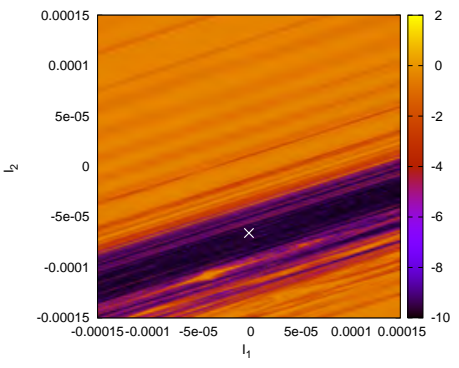

(b) $\epsilon=6 \times 10^{-3}$

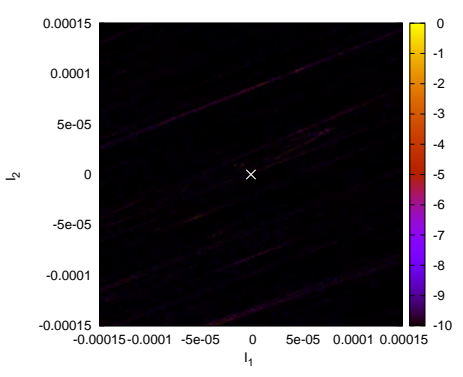

(e) $\epsilon=2 \times 10^{-2}$

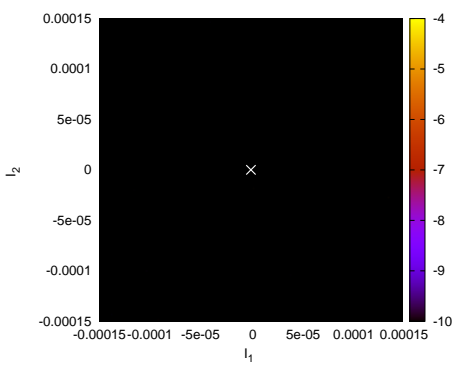

(h) $\epsilon=8 \times 10^{-2}$

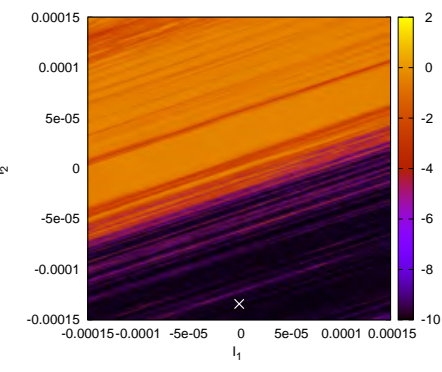

(c) $\epsilon=8 \times 10^{-3}$

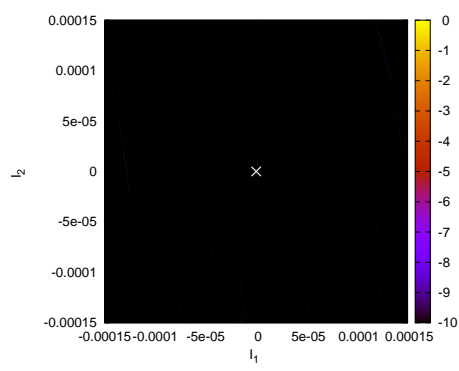

(f) $\epsilon=4 \times 10^{-2}$

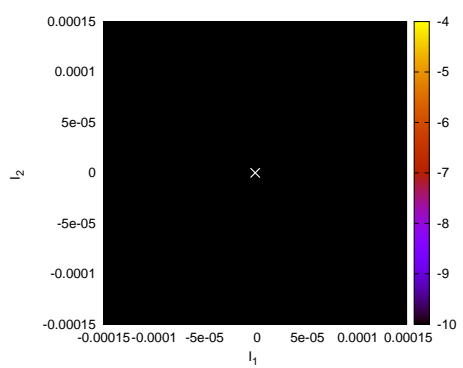

(i) $\epsilon=10^{-1}$

Figura 4.16: Entornos de la separatrices, para $\epsilon \in \mathscr{B}_{\star}$ y $h$ canónico, realizados durante el proceso de selección de las condiciones iniciales del ensamble de partículas prueba. La cruz blanca indica los centros de los correspondientes ensambles. Para cada valor de $\epsilon$, el origen de los ejes ha sido trasladado hacia la posición teórica de las respectivas separatrices.

la sección anterior. Salvo el caso de $\epsilon=2 \times 10^{-2}$, todos estos valores mencionados presentan una aparente estabilización en $10^{4} \leq t \leq 10^{6.5}$ (una vez superado el tiempo transitorio del crecimiento abrupto). Por otro lado, para $\epsilon$ igual $4 \times 10^{-2}$ (amarillo), $6 \times 10^{-2}$ (negro), $8 \times 10^{-2}$ (naranja) y $10^{-1}$ (gris) se observa un crecimiento "exponencial" o "super-exponencial" hasta la saturación en un único valor: $\sigma_{3}^{2} \approx 0.045$. Esta saturación se debe a que para $\epsilon \geq 4 \times 10^{-2}$, el sistema se halla en régimen de Chirikov por lo que los ensambles tienen acceso a la mayoría de la superficie energética. Para este último grupo de valores de $\epsilon$ se computó $\mathcal{D}_{\sigma}(\epsilon)$, utilizando $t_{0}=0 \mathrm{y}$ dos tiempos finales distintos, a pesar de que la varianza carece de comportamiento lineal. En la Fig. 4.18 se muestra con cuadrados vacíos el coeficiente para $t=1000$ y con cuadrados llenos el coeficiente para $t=2000$. Se observa que ambos intervalos de tiempo, 


\begin{tabular}{|r|c|c|}
\hline$\epsilon$ & $\mu_{1}(0)$ & $\mu_{2}(0)$ \\
\hline $4 \times 10^{-3}$ & 0.28719124745789 & 0.09011720000000 \\
$6 \times 10^{-3}$ & 0.28655999472904 & 0.09102000000000 \\
$8 \times 10^{-3}$ & 0.28602782406160 & 0.09175000000000 \\
$10^{-2}$ & 0.28555897229157 & 0.09245000000000 \\
$2 \times 10^{-2}$ & 0.28371943838387 & 0.09534676835013 \\
$4 \times 10^{-2}$ & 0.28111794458314 & 0.09924900905121 \\
$6 \times 10^{-2}$ & 0.27912174818079 & 0.10224330365474 \\
$8 \times 10^{-2}$ & 0.27743887676773 & 0.10476761077433 \\
$10^{-1}$ & 0.27595623728945 & 0.10699156999175 \\
\hline
\end{tabular}

Tabla 4.3: Centros de las condiciones iniciales de los ensambles, $\mu_{1}(0)=\left\langle I_{1}(0)\right\rangle$ y $\mu_{2}(0)=$ $\left\langle I_{2}(0)\right\rangle$, para $\epsilon \in \mathscr{B}_{\star}$.
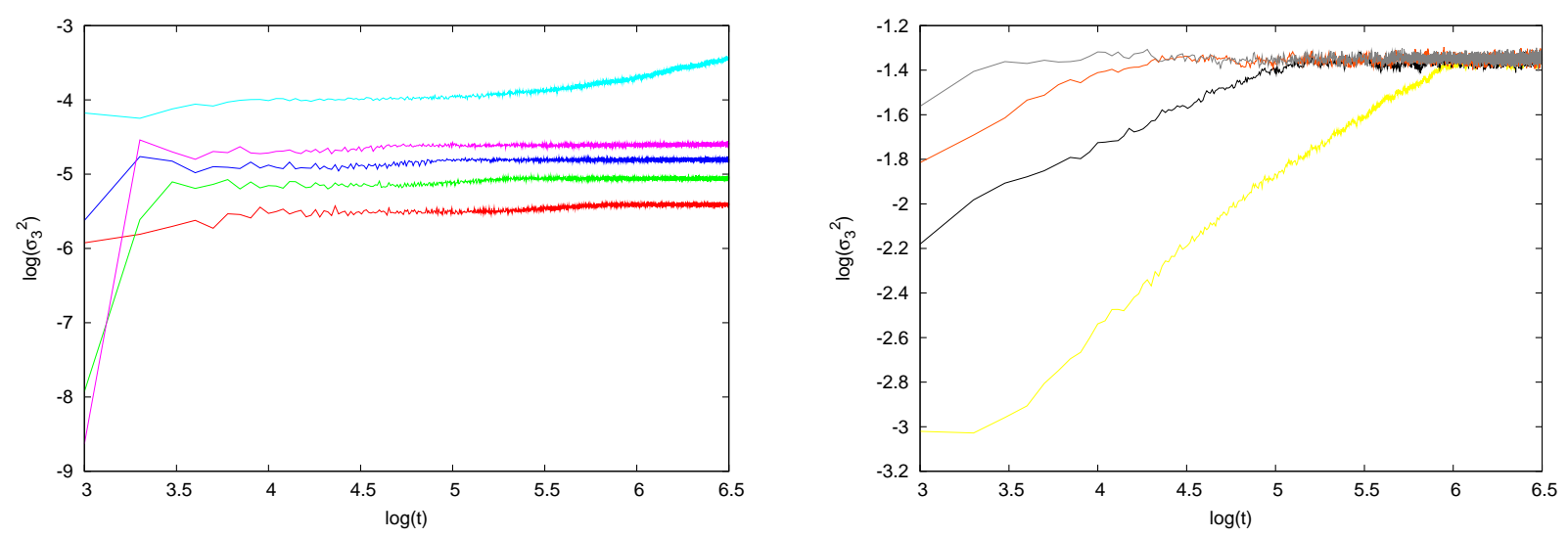

Figura 4.17: $\sigma_{3}^{2}(t)$ en escala logarítmica, para $\epsilon \in \mathscr{B}_{\star}$ y $t \in\left[10^{3}, 10^{6.5}\right]$, respectivamente en colores rojo, verde, azul, magenta, cyan, amarillo, negro, naranja y gris.

arrojan valores dentro del mismo orden de magnitud. A modo de poder comparar con los

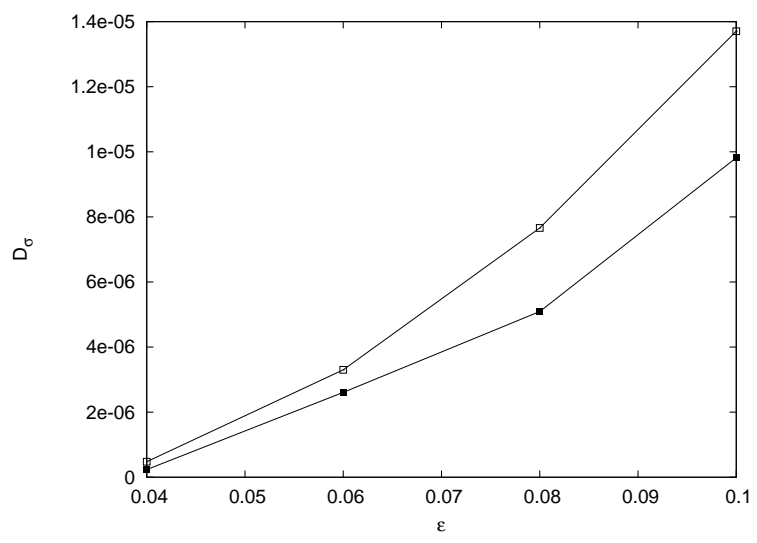

Figura 4.18: $\mathcal{D}_{\sigma}(\epsilon)$ utilizando $t_{0}=0 \mathrm{y}$ dos tiempos finales: $t=1000$ (cuadrados vacíos) y $t=2000$ (cuadrados llenos). 
coeficientes recientemente obtenidos y con los de $\mathscr{A}$, se ha computado nuevamente $\mathcal{D}_{\sigma}$ para $\epsilon$ igual a 0.04 y a 0.02 , utilizando el rango $10^{4} \leq t \leq 5 \times 10^{5}$, obteniéndose los valores $2.96 \times 10^{-8}$ y $6.63 \times 10^{-11}$, respectivamente. En la Fig. 4.19 se muestran, en escala semi-logarítmica, dichos valores (señalados con puntos triangulares) junto con los correspondientes a la Fig. 4.14 y a la Fig. 4.18. Se aprecia un desacuerdo de un orden de magnitud en el caso de $\epsilon=0.04$. Sin

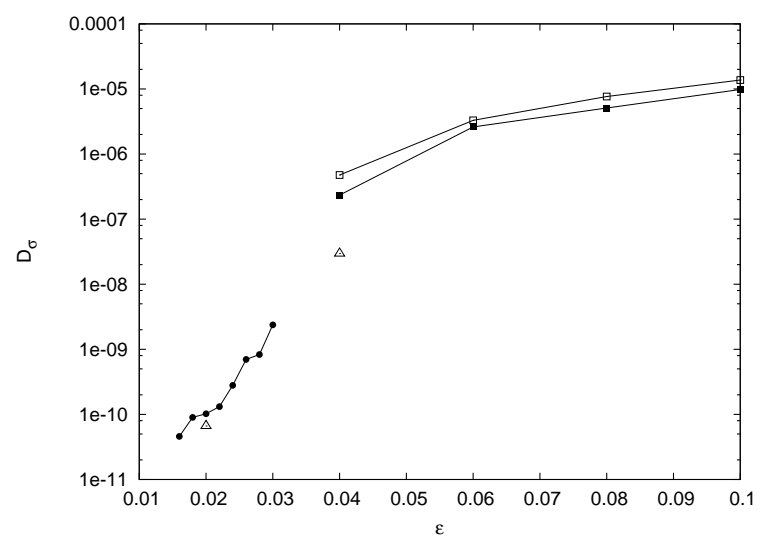

Figura 4.19: $\mathcal{D}_{\sigma}(0.02)$ y $\mathcal{D}_{\sigma}(0.04)$, utilizando $t_{0}=10^{4}$ y $t=5 \times 10^{5}$, señalados con triángulos. Para comparación se han agregado los datos de la Fig. 4.14 (círculos) y de la Fig. 4.18 (cuadrados).

embargo, para el caso de $\epsilon=0.02$, donde ambos coeficientes se computan en el mismo rango temporal, se obtiene concordancia en el orden de magnitud. Esto era esperable ya que, como fue antes mencionado, la acción resonante utilizada en $\mathscr{A}$ es muy próxima a la utilizada en $\mathscr{B}$, por lo que ambos coeficientes miden la difusión local en prácticamente la misma región.

Para estimar el coeficiente de difusión para los cuatro valores más pequeños de $\mathscr{B}_{\star}$, se debió extender el tiempo de integración, utilizando el integrador $S 6 a^{4}$ con un paso menor, $\Delta t_{i n t}=$ $10^{-1}$, alcanzándose un tiempo final de $2 \times 10^{8}$. En la Fig. 4.20 se muestra, en rojo, la evolución de las varianzas para $t \in\left[10^{7}, 2 \times 10^{8}\right]$. Se observa un comportamiento aproximadamente lineal, pero esta vez no se utilizó la fórmula (1.40). Para evitar que las oscilaciones de corto período arruinaran el cómputo de los coeficientes, se procedió a realizar un ajuste numérico de la forma:

$$
\sigma_{3}^{2}(t)=2 \mathcal{D}_{\sigma} t+c
$$

para $t \in\left[10^{7}, 2 \times 10^{8}\right]$, cuyo resultado se despliega en la Tabla 4.4. Dichos ajustes se muestran,

\begin{tabular}{|c|c|c|}
\hline$\epsilon$ & $\mathcal{D}_{\sigma}$ & $c$ \\
\hline $10^{-2}$ & $2.9062 \times 10^{-13}$ & $1.6963 \times 10^{-5}$ \\
$8 \times 10^{-3}$ & $1.9984 \times 10^{-14}$ & $1.5441 \times 10^{-5}$ \\
$6 \times 10^{-3}$ & $6.4117 \times 10^{-16}$ & $8.7747 \times 10^{-6}$ \\
$4 \times 10^{-3}$ & $<10^{-16}$ & $3.8848 \times 10^{-6}$ \\
\hline
\end{tabular}

Tabla 4.4: $\mathcal{D}_{\sigma}(\epsilon)$, ajustando ansatz (4.15) para $t \in\left[10^{7}, 2 \times 10^{8}\right]$.

\footnotetext{
${ }^{4}$ La confianza en la precisión de $S 6 a$ usando $\Delta t_{\text {int }}=10^{-1}$ fue reforzada al verificar que los valores de $\sigma_{3}^{2}$ obtenidos en esta integración concuerdan (salvo fluctuaciones de pequeño tamaño) durante las primeros $6 \times 10^{6}$ unidades de tiempo con los obtenidos usando el $S 8 b$ y paso $\Delta t_{\text {int }}=10^{-2}$.
} 


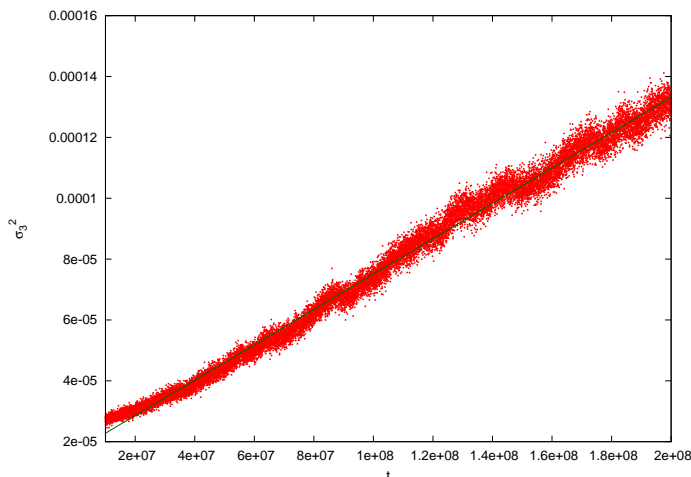

(a) $\epsilon=10^{-2}$

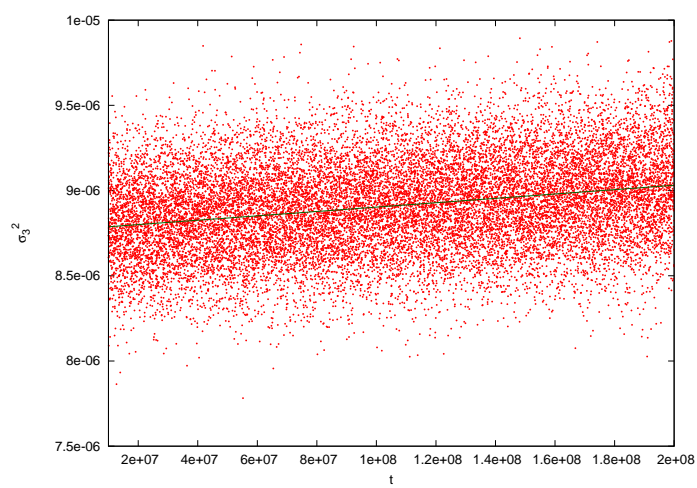

(c) $\epsilon=6 \times 10^{-3}$

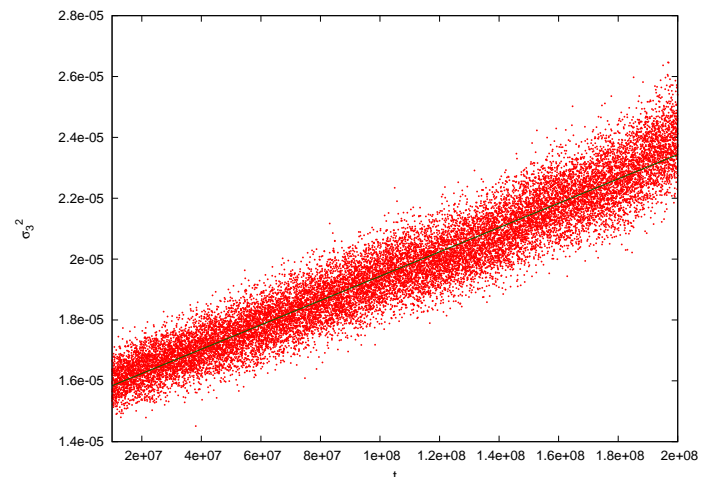

(b) $\epsilon=8 \times 10^{-3}$

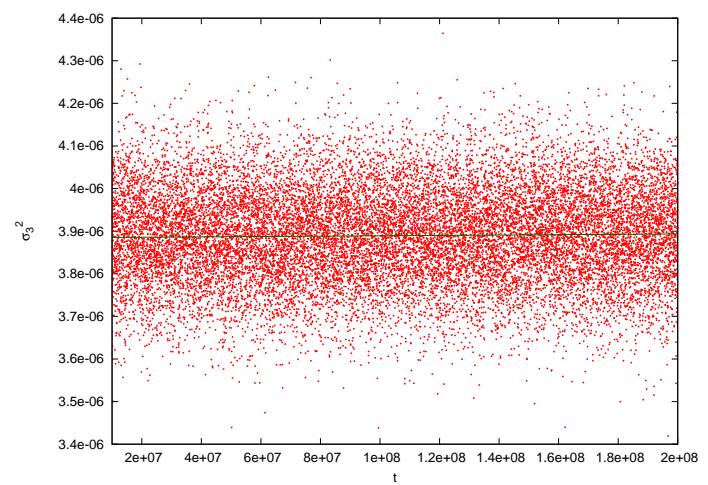

(d) $\epsilon=4 \times 10^{-3}$

Figura 4.20: $\sigma_{3}^{2}(t)$ para $10^{7} \leq t \leq 2 \times 10^{8}$ con $\epsilon=10^{-2}, 8 \times 10^{-3}, 6 \times 10^{-3}$ y $4 \times 10^{-3}$. Las rectas señalan la bondadez del ajuste numérico de $\mathcal{D}_{\sigma}$.

en color verde, en la misma figura.

Los valores de $\mathcal{D}_{\sigma}(\epsilon)$ recién computados se muestran, señalados con cruces, en la Fig. 4.21. El caso de $\epsilon=4 \times 10^{-3}$ ha sido omitido debido a que no presenta difusión en el intervalo temporal integrado. Con los datos disponibles en todas las mediciones realizadas hasta ahora no fue posible realizar una ajuste numérico de la relación (subsección 1.4.3):

$$
\mathcal{D}_{\sigma}(\epsilon)=a \exp \left(-\frac{b}{\epsilon^{c}}\right)
$$

Ello se debe a que el comportamiento exponencial predicho por Nekhorshev, no es numéricamente detectable si $\epsilon$ se mantiene tan cercano al $\epsilon_{c}$. Una situación similar se da para la resonancia y el sistema dados en Lega et al. (2003), para los cuales Efthymiopoulos (2008) justifica el motivo de que el comportamiento exponencial se haga evidente recién para $\epsilon$ un orden de magnitud menor al $\epsilon_{c}$. Por lo tanto, para ajustar $\mathcal{D}_{\sigma}(\epsilon)$ en dicho sistema, es necesario tener mediciones de $\epsilon$ hasta al menos dos órdenes de magnitud inferior al $\epsilon_{c}$. 


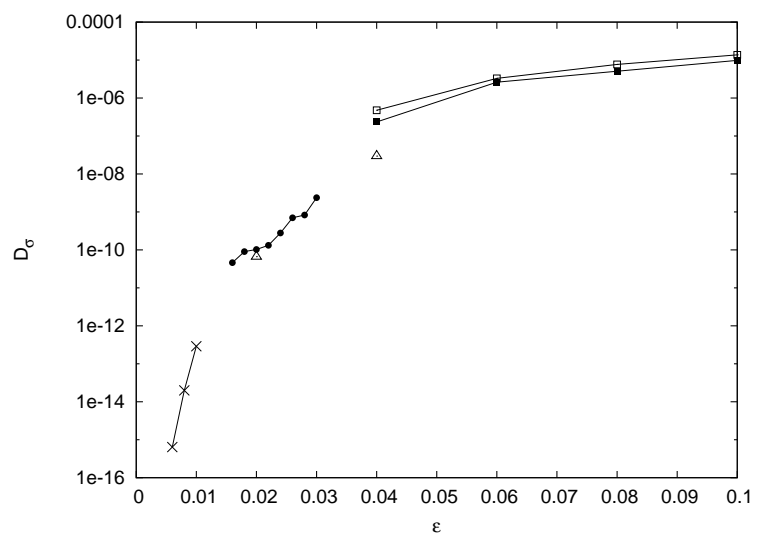

Figura 4.21: Valores de $\mathcal{D}_{\sigma}(\epsilon)$ tomados de Tabla 4.4, señalados con cruces, junto con los datos de la Fig. 4.19.

\subsection{Experimento $\mathscr{C}$}

En este último experimento con la difusión en el oscilador cuártico nos focalizaremos en un valor fijo del parámetro perturbativo, $\epsilon=0.02$, y dejaremos variar la ubicación de los ensambles iniciales a lo largo de la resonancia guía.

\subsubsection{Los ensambles}

La Fig. (4.22) muestra diez puntos de colores ubicados a lo largo de la "rama superior" de la capa estocástica asociada a la resonancia guía. Cada uno de ellos señala el centro de un ensamble de $10^{3}$ partículas elegidas aleatoriamente en entornos cuadrados de área $4 \times 10^{-12}$ sobre el plano $\left[I_{1}, I_{2}\right]$ y fijando todos los ángulos en $\pi / 2$. La Tabla 4.5 enumera dichos ensambles y brinda la información de la acción resonante utilizada y de la posición de sus centros geométricos iniciales. El procedimiento para la elección de tales centros fue similar a lo explicado en la sección anterior, es decir, a través de la exploración con amplificaciones de alta definición de la estructura resonante. La acción resonante utilizada para el quinto ensamble (cyan) es exactamente la misma que fue utilizada en el experimento $\mathscr{B}$, pero las condiciones iniciales de los respectivos ensambles fueron elegidas levemente diferentes.

\subsubsection{Las mediciones}

Los diez ensambles anteriormente definidos fueron integrados durante $10^{7}$ unidades de tiempo, utilizando el integrador $S 8 b$, un paso de integración $\Delta_{i n t}=10^{-1}$ y un paso de escritura $\Delta_{e s c}=10^{3}$. En la Fig. 4.23 se puede ver la evolución de $\sigma_{3}^{2}$, de los diez ensambles para $t_{T}=10^{6}$. Los colores de cada curva corresponden con los definidos en la Tabla 4.5. Se ve que existen seis ensambles cuya varianza evoluciona linealmente con el tiempo, salvo fluctuaciones de tamaño relativo menor, mientras que los otros 4 restantes presentan comportamientos claramente no lineales.

Se registra que los ensambles que poseen comportamiento normal en la difusión son aquellos cuyas condiciones iniciales se encuentran más lejos de las intersecciones de la resonancia guía con la $(2,-1,-1)$ y la $(2,0,-2)$. En otras palabras, los ensambles $4-9$ poseen comportamiento lineal de la varianza mientras que los ensambles 1 - 3 y 10 tienen una evolución de la 


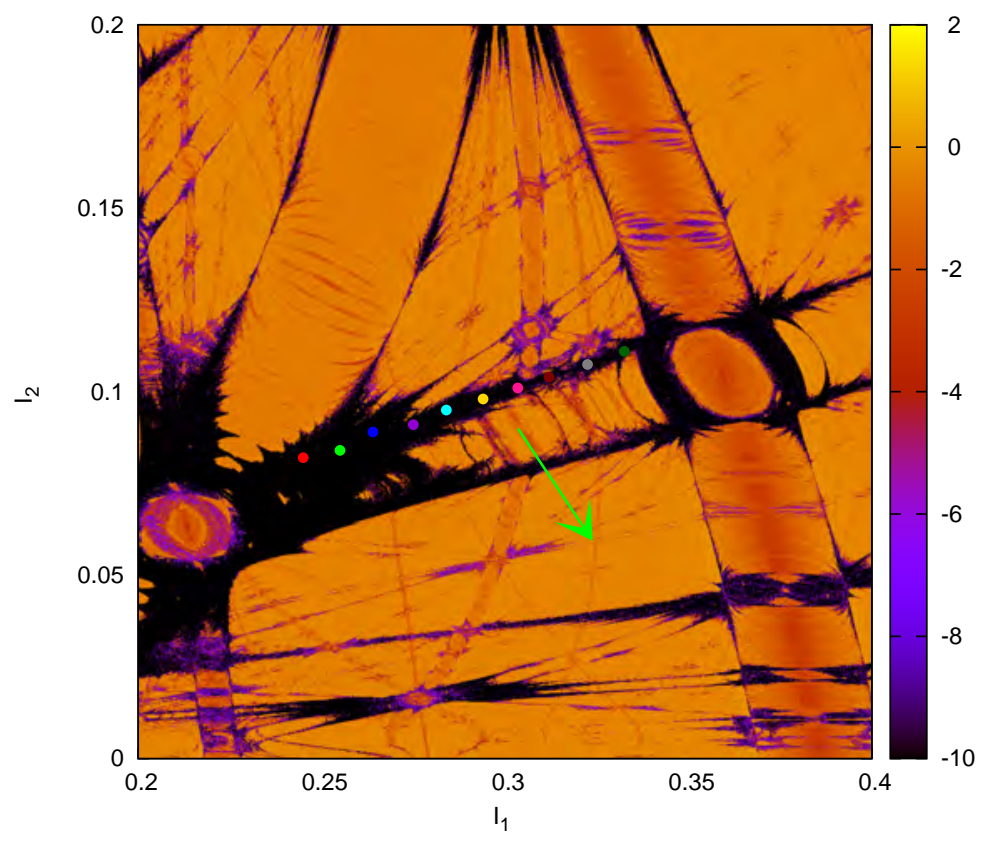

Figura 4.22: Ubicación de los ensambles del experimento $\mathscr{C}$, para $\epsilon=0.02$. La enumeración de los mismos está dada en Tabla 4.5. La flecha verde es paralela al vector $\boldsymbol{m}_{g}$.

\begin{tabular}{|c|c|c|c|c|}
\hline Ensamble & Color & $I_{1}^{r}$ & $\mu_{1}(0)$ & $\mu_{2}(0)$ \\
\hline 1 & Rojo & 0.25 & 0.2450 & 0.0820 \\
2 & Verde & 0.26 & 0.2550 & 0.0840 \\
3 & Azul & 0.27 & 0.2640 & 0.0890 \\
4 & Violeta & 0.28 & 0.2750 & 0.0910 \\
5 & Cyan & 0.29 & 0.2840 & 0.0950 \\
6 & Amarillo & 0.30 & 0.2940 & 0.0980 \\
7 & Magenta & 0.31 & 0.3035 & 0.1010 \\
8 & Marrón & 0.32 & 0.3120 & 0.1040 \\
9 & Gris & 0.33 & 0.3225 & 0.1074 \\
10 & Verde Oscuro & 0.34 & 0.3325 & 0.1110 \\
\hline
\end{tabular}

Tabla 4.5: Datos de ensambles del experimento $\mathscr{C}$. Los correspondientes valores de $I_{2}^{r}$ y de $I_{3}^{r}$ pueden ser calculados a partir de $I_{1}^{r}, \boldsymbol{m}_{g}, h$ y $A$, utilizando la Ec. (3.46).

varianza correspondiente a una difusión anómala. También se puede apreciar que, para (casi) todo tiempo fijo, todos los ensambles con comportamiento normal en la difusión mantienen sus varianzas con valores por debajo de aquellos correspondientes a los ensambles con difusión anómala.

Sin embargo, si miramos durante un período mayor de tiempo, como $t_{T}=10^{7}$, la situación es bastante diferente. En la Fig. 4.24 se puede ver que los ensambles $4-10$ son los que más han aumentado su varianza luego de $10^{7}$ unidades de tiempo. Por lo tanto, podría decirse que existe una tendencia general de que los ensambles ubicados en el entorno de dominios de resonancia doble posean una difusión inicial fuerte pero estable a tiempos grandes, mientras que los 
ensambles localizados en dominios de resonancias simples sufran una difusión, inicialmente lenta, pero eficiente a tiempos grandes. Para los seis ensambles cuya difusión es normal, se

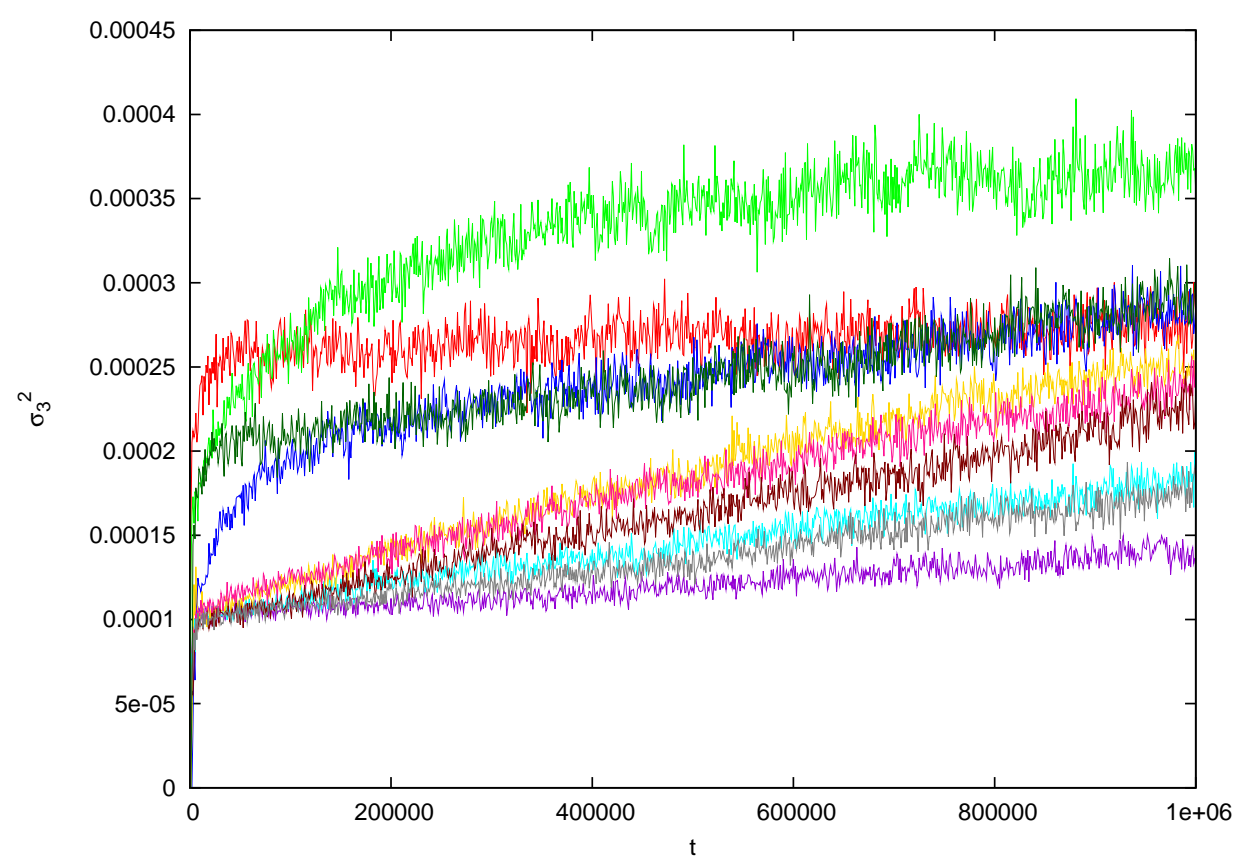

Figura 4.23: $\sigma_{3}^{2}(t)$, con $t \leq 10^{6}$, para los diez ensambles de Tabla 4.5

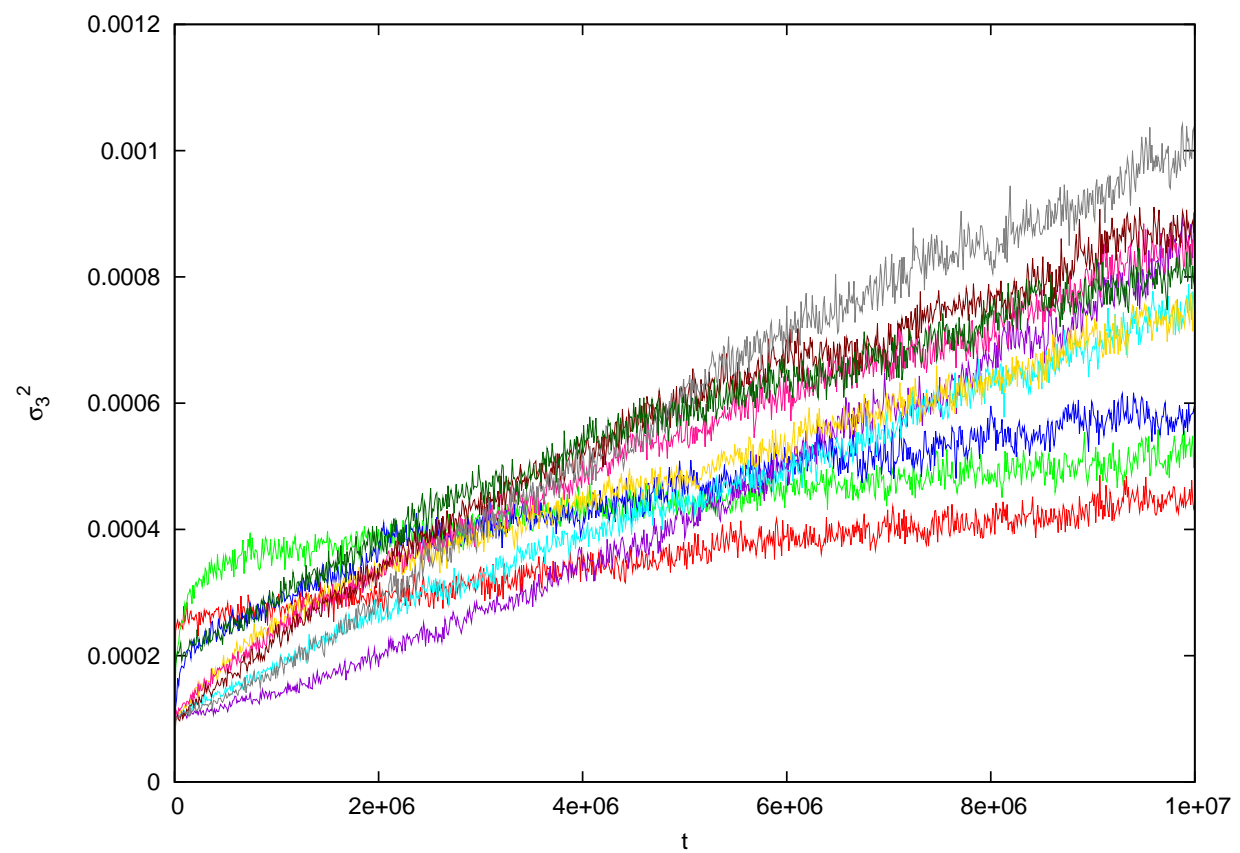

Figura 4.24: $\sigma_{3}^{2}(t)$, con $t \leq 10^{7}$, para los diez ensambles de Tabla 4.5

computó $\mathcal{D}_{\sigma}$, ajustando $(4.15)$ para $10^{4} \leq t \leq 10^{6}$. Los valores obtenidos fueron $1.95 \times 10^{-11}$, 
$4.04 \times 10^{-11}, 7.61 \times 10^{-11}, 6.54 \times 10^{-11}, 6.23 \times 10^{-11}$ y $3.85 \times 10^{-11}$, respectivamente para los ensambles $4-9$. Los mismos se despliegan en la Fig. 4.25, junto con aquellos coeficientes computados en experimentos anteriores, focalizando sólo en el rango $0.016 \leq \epsilon \leq 0.024$. Se

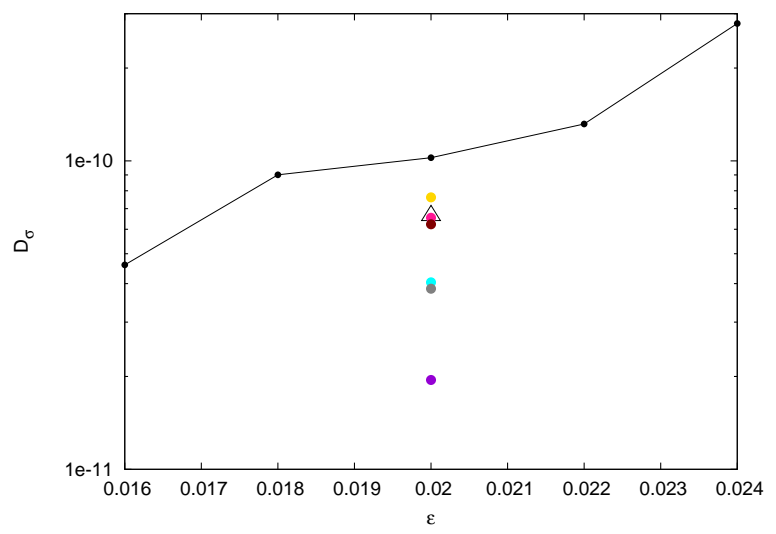

Figura 4.25: Valores de $\mathcal{D}_{\sigma}(\epsilon)$ para los ensambles $4-9$ de Tabla 4.5, señalados con los correspondientes colores, junto con los datos computados en experimentos anteriores en el rango $0.014 \leq \epsilon \leq 0.024$.

observa que los tres ensambles que más difunden (amarillo, magenta y marrón), son los que están más alejados de los cruces de resonancias. Además, se observa que el valor del coeficiente medido para el ensamble cyan difiere un poco del medido en $\mathscr{B}$ (señalado con un triángulo). Por otro lado, los ensambles magenta y marrón, dan valores del coeficiente cuyos puntos se ubican cercanos al triángulo, a pesar de que estos dos ensambles corresponden a acciones resonantes diferentes.

\subsection{Conclusión}

En este capítulo se estudió la difusión de Arnold que tiene lugar a lo largo de la capa estocástica de la resonancia guía $\boldsymbol{m}_{g}=(2,-3,0)$ del oscilador cuártico perturbado 3DoF. Las mediciones pueden agruparse en tres experimentos numéricos.

En el experimento $\mathscr{A}$ se estudió la difusión de ocho ensambles de partículas de prueba, uno por cada valor de $\epsilon \in \mathscr{A}_{\star}$. Se midió la evolución de las varianzas en las tres direcciones de la base de Chirikov, determinándose que para $\epsilon \leq 0.02$ se obtiene la situación típica esperable en un proceso de difusión de Arnold. Dicha situación está caracterizada por el hecho de que $\sigma_{1}^{2}(t)$ y $\sigma_{2}^{2}(t)$ se estabilizan aproximadamente en valores constantes, mientras que $\sigma_{3}^{2}(t)$ posee un crecimiento prácticamente monótono, en el intervalo temporal considerado: $t \in\left[0,10^{7}\right]$. Para valores de $\epsilon$ mayores, se obtiene que $\sigma_{1}^{2}(t)$ tiene una velocidad de crecimiento promedio que aumenta con $\epsilon$, en concordancia con el hecho de que el overlap con resonancias cercanas empieza a tener una incidencia cada vez mayor a medida que se aumenta $\epsilon$.

Para $t \lesssim 10^{7}$ se observó un comportamiento anómalo de $\sigma_{3}^{2}(t)$ en los ocho valores de $\epsilon$. Mediante ajustes del tipo de ley de potencias, se encontró que todos los exponentes son menores a uno (se mantienen por debajo de $w \sim 0.8$ ), lo cual es evidencia de que se comporta como un proceso subdifusivo en esta escala temporal. Por otro lado, para $t \lesssim 5 \times 10^{5}$ se observó que dichas varianzas siguen un comportamiento aproximadamente lineal (difusión normal) y se computaron los coeficientes de difusión asociados. 
En el experimento $\mathscr{B}$ se estudió la difusión de nueve ensambles de partículas de prueba, uno por cada valor de $\epsilon \in \mathscr{B}_{\star}$. Analizando la evolución de $\sigma_{3}^{2}(t)$, para $t \leq 10^{6.5}$, se observan dos comportamientos muy diferentes entre sí. Para $\epsilon \geq 0.04$ se evidencia un crecimiento de velocidad exponencial, hasta alcanzar un valor común de saturación de $\sigma_{3}^{2} \approx 0.045$, compatible con el hecho de que para estos valores el sistema se halla, efectivamente, en régimen de overlap generalizado. Para $\epsilon \leq 0.01$, las varianzas presentan una aparente estabilización, en valores mucho más pequeños que el de saturación. Recurriendo a diferentes estrategias, se computaron estimaciones numéricas del coeficiente de difusión. Sin embargo, no se intentó realizar un ajuste de la dependencia del coeficiente con $\epsilon$, debido a que para ello se necesitaría contar con mediciones para valores de $\epsilon$ mucho menores.

Finalmente, en el experimento $\mathscr{C}$ se midió $\sigma_{3}^{2}(t)$ para $\epsilon$ fijo en 0.02 , que corresponde con el régimen de Nekhoroshev. En este caso, se consideraron diez ensambles distribuidos a lo largo de un segmento de la capa estocástica asociada a la resonancia guía. Para $t \leq$ $10^{6}$ se observa que seis ensambles, cuyas condiciones iniciales son las más alejadas de los cruces con otras resonancias, presentan difusión normal; mientras que los restantes ensambles presentan comportamiento anómalo. Al considerar tiempos mayores, $t \leq 10^{7}$, se registra que, en general, aquellos ensambles ubicados en entornos de resonancias aisladas y alejados de los cruces, difunden a largo plazo, más eficientemente que aquellos ubicados cercanos a dichos cruces. Para los seis ensambles con difusión normal se calcularon los coeficientes de difusión, obteniéndose valores del orden de los obtenidos en experimentos anteriores para este valor del parámetro perturbativo. 


\section{El enfoque estocástico}

El objetivo general de este capítulo consiste en comprender algunos aspectos de la interrelación entre sistemas caóticos y procesos estocásticos. El objetivo específico consiste en caracterizar y probar una ecuación cinética para la función densidad de probabilidad de una acción, en un mapeo simple cuya geometría mantiene cierta semejanza con el problema de la difusión a lo largo de una resonancia.

Para ello, se realizará una aplicación empírica, sobre un mapa simpléctico $4 D$ a priori inestable, de un teorema proveniente de la teoría de procesos estocásticos. La motivación de esta idea es de origen fenomenológico y será explicada en la sección 5.4.1. Se mostrarán ejemplos de que existe un comportamiento difusivo descriptible en términos de la ecuación de FokkerPlanck y que existe un método numérico para predecir aproximadamente el correspondiente coeficiente de difusión. La estructura del capítulo se da a continuación.

En la sección 5.1 se describirá el teorema (y método) estocástico, originalmente desarrollado por Beccaceci (1996) para ser aplicado a sistemas Hamiltonianos integrables perturbados estocásticamente.

En la sección 5.2 se ejemplificará como funciona el método, mediante experimentos propios, en sistemas cuya parte integrable será la misma del sistema caótico que realmente nos interesa estudiar. Se utilizarán distintos tipos de ruidos Gaussianos en el papel de perturbaciones estocásticas. En 5.2.1 se utilizará ruido blanco Gaussiano. En las subsecciones 5.2.2, 5.2.3 y 5.2.4 se analizan tres tipos de ruidos coloreados Gaussianos. Uno de ellos consiste en un ruido generado mediante el filtrado de un proceso blanco Gaussiano bidimensional a través de una convolución particular. Los otros dos ruidos analizados son generados por ecuaciones diferenciales estocásticas con ruido Browniano aditivo y coeficientes constantes. Éstos son el proceso de Ornstein-Uhlenbeck y la dinámica de Langevin de un oscilador forzado, respectivamente. Además, en esta sección se definirán otras clases de coeficientes de difusión que serán utilizados tanto en los cómputos estocásticos como en los deterministas.

En la sección 5.3 se presentará el mapa simpléctico 4D de interés y se generalizará el método para computar el coeficiente de difusión, de forma de permitir una clase más amplia de perturbaciones.

En la sección 5.4 se aplicará el método para una geometría del problema que modela (con sencillez) una capa estocástica gruesa. Se verificará que este método permite predecir aproximadamente el coeficiente de difusión medido numéricamente y se demostrará empíricamente que la evolución de la función densidad de probabilidad de la acción (más específicamente 
una quasi-acción) se comporta como un proceso difusivo regido por una ecuación de FokkerPlanck.

En la sección 5.5 se aplicará el mismo procedimiento que en la sección anteriormente mencionada, considerando esta vez, una geometría que modela una capa estocástica delgada. De esta manera, se estará trabajando en el modelo de pumping estocástico para la difusión de Arnold en un mapa simpléctico a priori inestable.

\subsection{Un teorema de promediado}

A continuación se enunciará un teorema de averaging o promediado, para sistemas de tiempo discreto integrables perturbados estocásticamente, que provee de una ecuación de Fokker-Planck para la distribución en la variable acción.

El primer teorema de promediado para una ecuación diferencial estocásticamente perturbada fue establecido por Khasminskii (1966). Posteriormente, en Cogburn and Ellison (1992) se generaliza este resultado probando que bajo ciertas condiciones una integral primera del movimiento del sistema no perturbado converge débilmente (ver más adelante) a un proceso difusivo. Este teorema pudo ser extendido a computar el límite difusivo de la dinámica de las acciones de un sistema Hamiltoniano perturbado estocásticamente (Beccaceci, 1996).

Bazzani and Beccaceci (1998) utilizan esta extensión del teorema para estudiar la dinámica de un flujo Hamiltoniano integrable perturbado estocásticamente. Comprueban numéricamente una de las implicancias del teorema: para perturbaciones suficientemente chicas, la función densidad de probabilidad de la variable acción satisface una ecuación de Fokker-Planck cuyo coeficiente de difusión depende de la función de correlación del proceso estocástico. Además, muestran el efecto de las resonancias entre la densidad espectral del ruido y las frecuencias propias del sistema integrable, mediante el uso de ruido armónico. Bazzani and Mais (1998) aplican el mencionado teorema al estudio de la dinámica de un mapa simpléctico perturbado estocásticamente. Prueban que en el límite de ruido con amplitud pequeña, un ruido coloreado puede excitar una difusión local en el espacio de fases. A continuación se establece, sin demostración, el teorema tal como fue enunciado por dichos autores.

Sea $M: \mathbb{R}^{2} \rightarrow \mathbb{R}^{2}$ un mapa simpléctico con un punto fijo elíptico en el origen y sea $\mathcal{U}$ un entorno de dicho origen que define una región estable. Se asume que en el entorno $\mathcal{U}$ la medida de las resonancias no lineales y de las regiónes caóticas es despreciable por lo que es plausible reemplazar al mapa inicial por uno integrable, $M_{0}: \mathbb{R}^{2} \rightarrow \mathbb{R}^{2}$. Sea además, $\mathbf{x} \equiv(x, p) \in \mathbb{R}^{2}$.

Se considera un mapa simpléctico estocástico, $P_{n}$, de la forma

$$
P_{n}(\mathbf{x})=\mathbf{x}+\epsilon \xi_{n} \mathbf{v}(\mathbf{x})
$$

donde $n \in \mathbb{N}, \xi_{n}$ es un proceso estocástico estacionario definido en algún espacio de probabilidad tal que $\left\langle\xi_{n}\right\rangle=0 \mathrm{y}$

$$
\mathbf{v}(\mathbf{x})=\left(\begin{array}{c}
0 \\
-\frac{d \tilde{V}(x)}{d x}
\end{array}\right)
$$

Luego, se estudiará la dinámica del siguiente mapa simpléctico estocásticamente perturbado:

$$
\mathbf{x}_{n+1}=P_{n} \circ M_{0}\left(\mathbf{x}_{n}\right),
$$

donde "o" denota la operación de composición. 
Introduciendo las variables ángulo-acción $(I, \theta)$ del mapa $M_{0}$, se reescribe el mapa (5.3) en la forma

$$
\left(\begin{array}{c}
\theta_{n+1} \\
I_{n+1}
\end{array}\right)=\exp \left(\epsilon \xi_{n} L_{V(I, \theta)}\right) \circ\left(\begin{array}{c}
\theta_{n}+\Omega\left(I_{n}\right) \\
I_{n}
\end{array}\right),
$$

donde $V \equiv V(I, \theta)=\tilde{V}(x(I, \theta)), L_{V}$ es el operador de Lie definido por el corchete de Poisson $\{\cdot, V\}$ con el potencial $V(x)$ y $\Omega(I)$ es la frecuencia del mapa $M_{0}$.

Se precisará el desarrollo de Fourier del potencial:

$$
V(I, \theta)=\sum_{k} V_{k}(I) e^{i k \theta}
$$

Para un valor fijo de $\epsilon$ introducimos una frecuencia de corte $k_{\text {max }}$ en la expansión (5.5) al despreciar los términos tales que $\left\|V_{k}\right\| \leq \epsilon$, donde $\|\cdot\|$ denota la norma supremo o infinito en el entorno $\mathcal{U}$.

Las hipótesis del teorema son las siguientes:

1. El mapa inicial (no perturbado) es al menos de clase $\mathcal{C}^{2}$ en $\mathcal{U}$.

2. Se considera que todas las partículas que arriban a la frontera $\partial \mathcal{U}$ del dominio de estabilidad son dispersadas, son eliminadas, por lo cual se simula el efecto introduciendo una barrera absorbente en $\partial \mathcal{U}$.

3. El ruido $\xi_{n}$ satisface una condición de mezclado- $\varphi$; es decir, si $f(x)$ y $g(x)$ son funciones medibles acotadas entonces la siguiente desigualdad se cumple

$$
\left|E\left[f\left(\xi_{n}\right) g\left(\xi_{0}\right)\right]-E\left[f\left(\xi_{n}\right)\right] E\left[g\left(\xi_{0}\right)\right]\right| \leq\|f|\||| g\| \varphi(n) ; \quad n \in \mathbb{N}
$$

donde la función $\varphi(n)$ es tal que $\operatorname{lím}_{n \rightarrow \infty} n^{6} \varphi(n)=0$ y $E[\cdot]$ es el valor de expectación (1.73).

4. No se cumple ninguna condición resonante $k \Omega(I)-2 \pi q=0(q \in \mathbb{Z})$ para $|k|<2 k_{\text {max }}$ $(k \neq 0)$ en la región $\mathcal{U}$ cuando $V_{k}(I) \neq 0$ en la expansión (5.5).

5. El siguiente límite existe:

$$
\mathcal{D}(I) \equiv \lim _{N \rightarrow \infty} \frac{1}{N} \sum_{n=0}^{N-1} \sum_{m=0}^{N-1} \frac{\partial V}{\partial \theta_{0}}\left(I, \theta_{0}+\Omega(I) n\right) \frac{\partial V}{\partial \theta_{0}}\left(I, \theta_{0}+\Omega(I) m\right) E\left[\xi_{n} \xi_{m}\right],
$$

donde se ha utilizado la notación

$$
\frac{\partial V}{\partial \theta_{0}}\left(I, \theta_{0}+\Omega(I) n\right) \equiv \frac{\partial V}{\partial \theta}(I, \theta)_{\mid \theta=\theta_{0}+\Omega(I) n} .
$$

El significado de la tercera suposición es que el proceso $\xi_{n}$ esta perdiendo memoria de su historia y la función $\varphi(n)$ da una medida de la independencia entre el futuro y el pasado; esta condición es necesaria si se quiere aproximar la dinámica en la acción con un proceso difusivo. El cuarto requisito evita la aparición de resonancias entre el movimiento no perturbado y la componente determinista de la perturbación, $V(I, \theta)$. Resonancias de este tipo introducirían discontinuidades en el coeficiente de difusión de la acción, efecto que aún no es comprendido. Debido a que se introdujo un truncamiento en la expansión de Fourier de $V(I, \theta)$ en un $k_{\max } \mathrm{y}$ 
a la condición de ausencia de resonancia en $\mathcal{U}$, se tiene que este coeficiente de difusión resulta ser independiente del ángulo inicial $\left(\theta_{0}\right)$.

Bajo estas hipótesis recién mencionadas vale la siguiente tesis.

Introduciendo el tiempo lento $L=\epsilon^{2} n$, en el límite $\epsilon \rightarrow 0$ el proceso estocástico $I_{\epsilon}(L) \equiv$ $I\left(L / \epsilon^{2}\right)$ converge débilmente en $\mathcal{U}$ hacia un proceso de difusión $\hat{I}(L)$ con una barrera absorbente en $\partial \mathcal{U}$ cuya densidad de probabilidad $\rho(\hat{I}, L)$ satisface la ecuación de Fokker-Planck ${ }^{1}$ :

$$
\frac{\partial \rho}{\partial L}(\hat{I}, L)=\frac{1}{2} \frac{\partial}{\partial \hat{I}}\left\{\mathcal{D}(\hat{I}) \frac{\partial \rho}{\partial \hat{I}}(\hat{I}, L)\right\}
$$

El concepto de convergencia débil debe entenderse como convergencia de la función densidad de probabilidad, definición que se clarifica a continuación y que no tiene relación con las realizaciones individuales del proceso. Sea $\rho(I, 0)$ una distribución inicial satisfecha por el ensamble que se evolucionará según el mapa (5.4), para un dado valor finito de $\epsilon$. Tal evolución otorgará, para cada tiempo $t$, una distribución $\rho_{\epsilon}(I, t)$. Por otro lado, la misma distribución inicial, evolucionada según la ecuación de F-P (5.9) dará, para cada tiempo $L$, la distribución $\rho(I, L)$. La convergencia de la función densidad de probabilidad significa que para cada $I$ y $L$ fijos, vale el siguiente límite:

$$
\lim _{\epsilon \rightarrow 0} \rho_{\epsilon}\left(I, L / \epsilon^{2}\right)=\rho(I, L)
$$

De Ecs. (5.9) y (5.10) se tiene que, para $\epsilon$ suficientemente chico, $\rho_{\epsilon}(I, t)$ satisface (aproximadamente) la siguiente ecuación de F-P:

$$
\frac{\partial \rho_{\epsilon}}{\partial t}(I, t)=\frac{1}{2} \frac{\partial}{\partial I}\left\{\epsilon^{2} \mathcal{D}(I) \frac{\partial \rho_{\epsilon}}{\partial I}(I, t)\right\}
$$

En estas circunstancias, $\mathcal{D}(I)$ es un coeficiente de difusión reescaleado y, antes de computarlo analíticamente, se introducen dos definiciones.

Una de ellas es la función de autocorrelación del ruido $\phi(n, m) \equiv E\left[\xi_{n}, \xi_{m}\right]$. Debido a la propiedad estacionaria del ruido, se tiene que $\phi(n, m)=\phi(n-m, 0) \quad \forall n, m \in \mathbb{N}$, por lo que en algunas oportunidades se utilizará la notación $\phi(n) \equiv \phi(n, 0)$.

La otra cantidad es la densidad espectral:

$$
\tilde{\phi}(\nu)=\sum_{j=-\infty}^{\infty} \phi(|j|) e^{i j \nu}
$$

Luego, utilizando la cuarta hipótesis junto con la expansión de Fourier (5.5), vale la siguiente aproximación:

\footnotetext{
${ }^{1}$ Esta ecuación corresponde a un caso particular de la Ec. (1.65), en el cual el coeficiente de drift, A, se relaciona con el de difusión, $\mathcal{D} \equiv B$, de la forma: $A=\frac{1}{2} \partial_{\hat{I}} \mathcal{D}$
} 


$$
\begin{aligned}
\mathcal{D}(I)= & \lim _{N \rightarrow \infty} \frac{\epsilon}{N} \sum_{j=-N+1}^{N-1} \sum_{n=j}^{j+N-1} \frac{\partial V}{\partial \theta_{0}}\left(I, \theta_{0}+\Omega(I) n\right) \frac{\partial V}{\partial \theta_{0}}\left(I, \theta_{0}+\Omega(I)(n-j)\right) \phi(|j|) \\
\approx & -\sum_{k}^{|k| \leq k_{\max }} \sum_{k^{\prime} \mid \leq k_{\max }} V_{k}(I) \bar{V}_{k^{\prime}}(I) k k^{\prime} e^{i\left(k-k^{\prime}\right) \theta_{0}} \\
& \times\left[\lim _{N \rightarrow \infty} \sum_{j=-N+1}^{N-1} \phi(|j|) e^{i k^{\prime} \Omega(I) j} \frac{1}{N} \sum_{n=j}^{j+N-1} e^{i\left(k-k^{\prime}\right) \Omega(I) n}\right] \\
= & \sum_{k}^{|k| \leq k_{\max }} k^{2}\left|V_{k}(I)\right|^{2} \hat{\phi}(k \Omega(I)),
\end{aligned}
$$

que es válida salvo por un error de $\mathcal{O}(\epsilon)$ debido a la presencia del truncamiento. Finalmente, se define el coeficiente de difusión analítico como:

$$
\mathcal{D}_{a}(I) \equiv \sum_{k}^{|k| \leq k_{\max }} k^{2}\left|V_{k}(I)\right|^{2} \hat{\phi}(k \Omega(I)) .
$$

En Bazzani and Mais (1998) se remarca la importancia de la densidad espectral $\hat{\phi}(\nu)$ en el comportamiento del coeficiente de difusión. En el límite difusivo $\epsilon \rightarrow 0$ sólo las componentes espectrales $k \Omega(I)\left(k \in \mathbb{Z},|k| \leq k_{\max }\right)$ del ruido contribuyen al coeficiente de difusión y ninguna difusión es observada cuando estas componentes se anulan.

\subsection{Ejemplos estocásticos con ruidos Gaussianos}

Nuestro mapa simpléctico perturbado estocásticamente es de la forma

$$
\begin{aligned}
& I_{n+1}=I_{n}-\epsilon \frac{\partial H_{1}}{\partial \theta_{n}}\left(I_{n+1}, \theta_{n}, \xi_{n}\right) \\
& \theta_{n+1}=\theta_{n}+\Omega\left(I_{n+1}\right)=\theta_{n}+I_{n+1} \quad \bmod 2 \pi
\end{aligned}
$$

donde

$$
H_{1}(I, \theta, \xi)=\xi V(\theta)=\xi \cos \theta,
$$

y $\xi_{n}$ es el valor de una realización particular del ruido al tiempo $n$. En otras palabras, nuestro sistema es un rotador libre, con ángulo $\theta$ y velocidad angular $\Omega(I) \equiv I$, perturbado por un término estocástico.

A continuación se definen dos coeficientes de difusión semi-numéricos. Dado un valor de la acción $I$, un ángulo inicial $\theta_{0} \mathrm{y}$ un valor finito pero suficientemente grande del tiempo final $N$, se define uno de los coeficientes semi-numéricos como la cantidad:

$$
\mathcal{D}_{s n}(I ; N) \equiv \frac{1}{N} \sum_{n=0}^{N-1} \sum_{m=0}^{N-1} \frac{\partial V}{\partial \theta_{0}}\left(\theta_{0}+\Omega(I) n\right) \frac{\partial V}{\partial \theta_{0}}\left(\theta_{0}+\Omega(I) m\right) \phi(n, m) .
$$

En caso de no disponer de la función $\phi(n, m)$, es posible reemplazarla por una autocorrelación numérica (promedio de ensambles), computando la siguiente variante del coeficiente 
semi-numérico:

$$
\mathcal{D}_{s n}^{\star}(I ; N) \equiv \frac{1}{N} \sum_{n=0}^{N-1} \sum_{m=0}^{N-1} \frac{\partial V}{\partial \theta_{0}}\left(\theta_{0}+\Omega(I) n\right) \frac{\partial V}{\partial \theta_{0}}\left(\theta_{0}+\Omega(I) m\right)\left\langle\xi_{n} \xi_{m}\right\rangle .
$$

Obtener el valor de $\mathcal{D}_{s n}^{\star}(I ; N)$ implica no sólo elegir valores de $I, \theta_{0}$ y $N$, si no también elegir el número de realizaciones del ruido $N_{r}$ que serán numéricamente computadas.

Como el desarrollo de Fourier de nuestro potencial es:

$$
V(\theta)=\cos (\theta)=\frac{1}{2} e^{i \theta}+\frac{1}{2} e^{-i \theta},
$$

se tiene que

$$
V_{k}(I)= \begin{cases}\frac{1}{2} & \text { si } k= \pm 1 \\ 0 & \text { si } k \neq \pm 1\end{cases}
$$

La existencia de sólo dos términos, y del mismo tamaño, en el desarrollo, implica que no es necesario adoptar valor de corte $\left(k_{\max }\right)$ alguno. Además, junto con la cuarta hipótesis del teorema de la sección 5.1 y el hecho de que $\Omega(I) \equiv I$, se tiene que en nuestro caso:

$$
\mathcal{U}=\{I \in \mathbb{R}: \quad I \neq 2 \pi q, \quad q \in \mathbb{Z}\} .
$$

Luego, se arriba a que la expresión analítica (5.13) toma la forma

$$
\mathcal{D}_{a}(I)=\frac{1}{4}\{\tilde{\phi}(-I)+\tilde{\phi}(I)\} .
$$

Ya se han definido previamente cuales serán las cantidades de predicción de coficientes de difusión: $\mathcal{D}_{a}(I), \mathcal{D}_{s n}(I ; N)$ y $\mathcal{D}_{s n}^{\star}(I ; N)$, que serán utilizadas durante el resto del capítulo. Estos valores serán oportunamente comparados contra la medición de un coeficiente de difusión numérico. El mismo será medido a través del desplazamiento cuadrático medio, a diferencia de lo que fue realizado en el capítulo precedente, donde se utilizó la varianza. Se medirá $\mathcal{D}_{\gamma}$, utilizando la convención asociada a la Ec. (1.65). Con los cambios de notación de $t_{0}, t, I\left(t_{0}\right)$ e $I(t)$, respectivamente por $0, N, I$ e $I_{N}$, se tiene que

$$
\mathcal{D}_{\gamma}(I ; N)=\frac{\left\langle\left(I_{N}-I\right)^{2}\right\rangle}{N}
$$

Finalmente, reescaleando esta última expresión, se define el coeficiente de difusión numérico (para $N$ unidades de tiempo), como:

$$
\mathcal{D}_{n u}(I ; N) \equiv \frac{1}{\epsilon^{2}} \mathcal{D}_{\gamma}(I ; N)=\frac{\left\langle\left(I_{N}-I\right)^{2}\right\rangle}{\epsilon^{2} N} .
$$

Para el cómputo de este coeficiente se utilizará, en la mayoría de los casos, el valor $N=10^{3}$. Para el conjunto de valores de $\epsilon$ que se utilizarán en este capítulo, dicho valor de $N$ es suficientemente pequeño como para que la difusión sea normal (debido a que el ensamble no se alcanza a alejar de su acción inicial) y suficientemente grande como para que las oscilaciones de corto período sean despreciables.

A continuación se ejemplificará el funcionamiento del método utilizando ruido blanco y tres tipos de ruidos coloreados. Se le llama ruido blanco a aquel ruido cuya densidad espectral es constante, o equivalentemente, que su autocorrelación está descripta por la distribución $\delta$ de Dirac. Por otro lado, se le llama ruido coloreado a todo ruido que no es blanco. Los ruidos coloreados que se verán a continuación estarán caracterizados por tener una o más frecuencias características, en el entorno de las cuales la densidad espectral poseerá máximos. 


\subsubsection{Ruido blanco}

En el caso de ruido Gaussiano $\delta$-correlacionado, con desviación estándar $\sigma_{w}=1$, la distribución de autocorrelación es $\phi(t)=\sigma_{w}^{2} \delta(t)=\delta(t)$. Esto implica $\tilde{\phi}(x)=\sigma_{w}^{2}=1$. Así el coeficiente analítico resulta ser:

$$
\mathcal{D}_{a}(I)=\frac{1}{4}\{\tilde{\phi}(-I)+\tilde{\phi}(I)\}=\frac{\sigma_{w}^{2}}{2}=\frac{1}{2} .
$$

El coeficiente semi-numérico $\mathcal{D}_{s n}$ no puede computarse debido a que la función autocorrelación es una función singular.

\subsubsection{Ruido generado mediante convolución de ruidos blancos.}

Ahora se verá el efecto, sobre el coeficiente de difusión, de un ruido generado mediante el filtrado de un proceso blanco Gaussiano bidimensional, que ha sido utilizado por Bazzani and Mais (1998). En esta oportunidad, sólo se computará el coeficiente semi-numérico junto con la densidad espectral, con el objetivo de estudiar el efecto de cambiar dos parámetros del ruido asociados a la frecuencia característica y al tiempo de correlación, pero no se contrastará con la medición numérica. Sean $w_{1}(t)$ y $w_{2}(t)$ ruidos blancos Gaussianos independientes de igual varianza, $\sigma_{w}^{2}$. Eso significa que $\left\langle w_{i}(t) w_{j}(s)\right\rangle=\sigma_{w}^{2} \delta_{i j} \delta(t-s) ; i, j=1,2$. Sea también el siguiente proceso:

$$
\xi_{t}=\frac{\sigma_{w}}{(\sqrt{2 \pi} \eta)^{1 / 2}} \int_{-\infty}^{\infty}\left(w_{1}(s) \cos \left[\omega_{0}(t-s)\right]+w_{2}(s) \sin \left[\omega_{0}(t-s)\right]\right) e^{-t^{2} / 4 \eta^{2}} d s
$$

El mismo es estacionario, con valor medio nulo y función de autocorrelación:

$$
\phi(\tau)=\sigma_{w}^{2} \cos \left(\omega_{0} \tau\right) e^{-\tau^{2} / 8 \eta^{2}}
$$

Utilizando las ecuaciones $(5.12),(5.27)$ y pidiendo la condición $\sigma_{w}=1$ la densidad espectral se expresa así:

$$
\tilde{\phi}(\nu)=\sum_{m=-\infty}^{\infty} \cos \left(\omega_{0} m\right) e^{-m^{2} / 8 \eta^{2}} e^{-i m \nu} .
$$

Es posible graficar, aproximadamente, la densidad espectral evaluando la parte real de la expresión (5.28), para un número finito de términos. Luego, se ha evaluado numéricamente la fórmula:

$$
\tilde{\phi}(\nu) \approx \sum_{m=-N}^{N} \cos \left(\omega_{0} m\right) e^{-m^{2} / 8 \eta^{2}} \cos (m \nu)
$$

para $\eta=50, \omega_{0}=0.3$ y $N=10^{3}$, y el resultado se despliega con trazo rojo en la Fig. 5.1 para $\nu \in[0.2,0.4]$ (izquierda) y para $\nu \in[-0.5,0.5]$ (derecha). Además, en ambas imágenes se ha trazado en negro la parte imaginaria de la evaluación numérica de $\tilde{\phi}(\nu)$, verificando que es nula. De las mismas se concluye que existen máximos centrados en $\nu= \pm \omega_{0}= \pm 0.3$. En la Fig. 5.2-izq. se compara el efecto de cambiar la frecuencia del ruido un orden de magnitud, tomando $\omega_{0}=0.03$. El resultado consiste en que los máximos cambian hacia $\nu= \pm 0.03$, mientras que el ancho y alto de la curva no perciben modificaciones. Asimismo, en la Fig. 5.2der. se compara el efecto de cambiar el tiempo de correlación un orden de magnitud, tomando $\eta=5$ y $\omega_{0}=0.3$. En este caso, la función se vuelve más ancha y pequeña, mientras que los máximos permanecen en sus valores originales. 

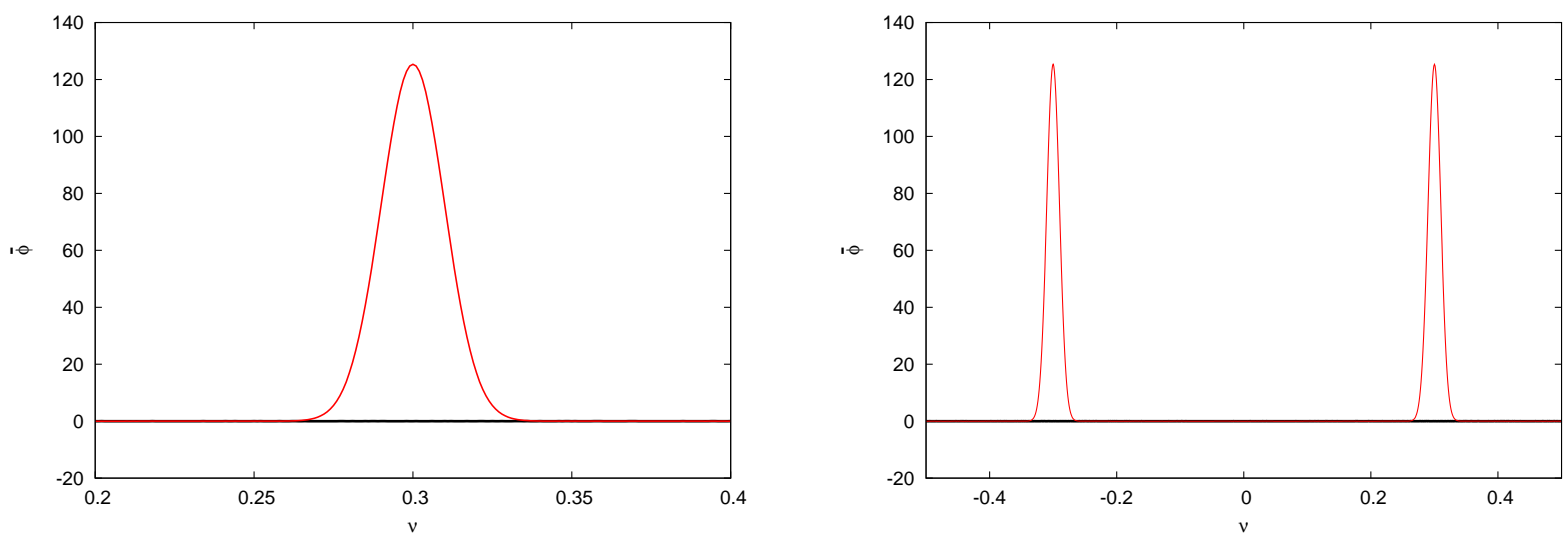

Figura 5.1: $\operatorname{Re}[\tilde{\phi}(\nu)]$ (rojo) y $\operatorname{Im}[\tilde{\phi}(\nu)]$ (negro) para $\sigma_{w}=1, \eta=50, \omega_{0}=0.3$ y $N=10^{3}$.
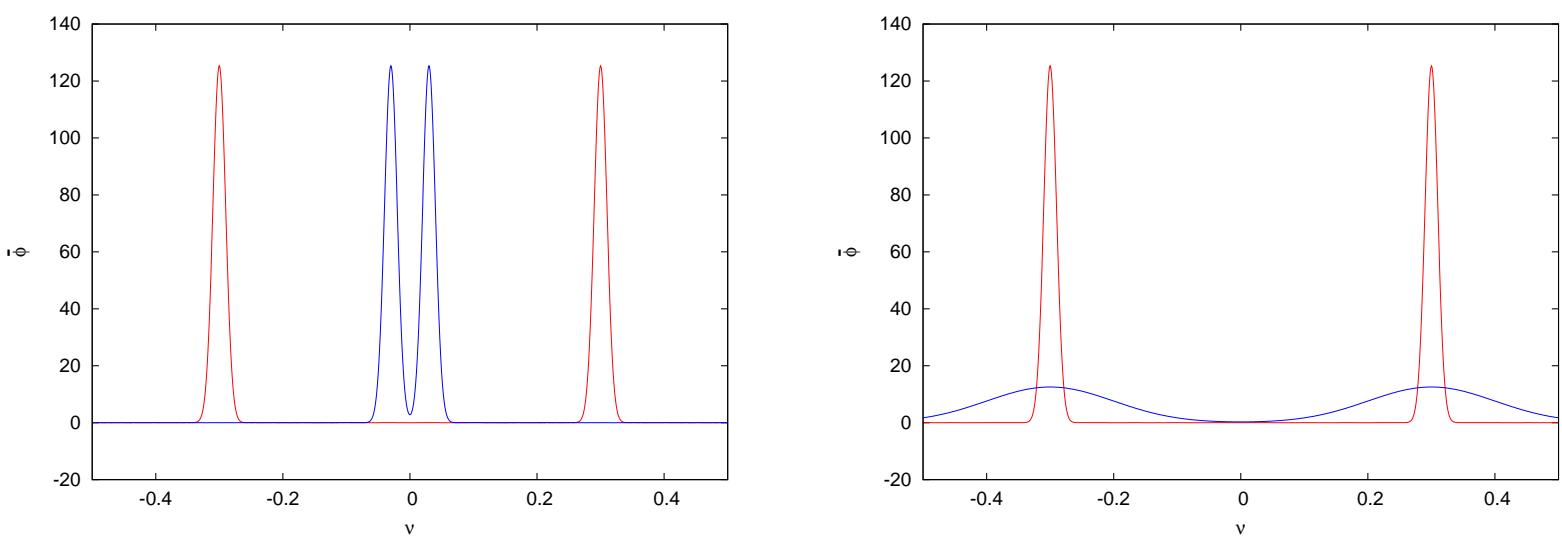

Figura 5.2: A la izq. se grafica $\tilde{\phi}(\nu)$ para $\eta=50, N=10^{3}$, utilizando $\omega_{0}=0.3$ (rojo) y $\omega_{0}=0.03$ (azul). A la der. se grafica $\tilde{\phi}(\nu)$ para $\omega_{0}=0.3, N=10^{3}$, utilizando $\eta=50$ (rojo) y $\eta=5$ (azul).

Introduciendo la expresión de $\phi(\tau)$ (5.27) en el algorítmo semi-numérico (5.17), se ha computado el coeficiente de difusión para $\omega_{0}=0.5$ y $\eta=50$, eligiendo un ángulo inicial al azar entre 0 y $2 \pi$, que resultó ser $\theta_{0}=0.21435176600573$. Esta cantidad es mostrada en la Fig. 5.3 para tres tiempos totales, $N=10^{3}, 3 \times 10^{3}, 5 \times 10^{3}$, respectivamente en colores rojo, azul y negro. En la figura de la derecha se ha utilizado escala logarítmica en el eje vertical. En ambas figuras se observa como repercute en $\mathcal{D}_{s n}(I ; N)$ el valor de $N$ elegido. Además, se observa que para cada valor fijo de $I$, el método tiende a converger al incremetar $N$, sobre todo en la región donde dicho coeficiente es considerablemente distinto de cero.

\subsubsection{Proceso Ornstein-Uhlenbeck}

A continuación se analizará el efecto sobre la difusión que tiene un ruido que consiste en un proceso de Ornstein-Uhlenbeck (O-U). El mismo ha sido extensamente utilizado en la 

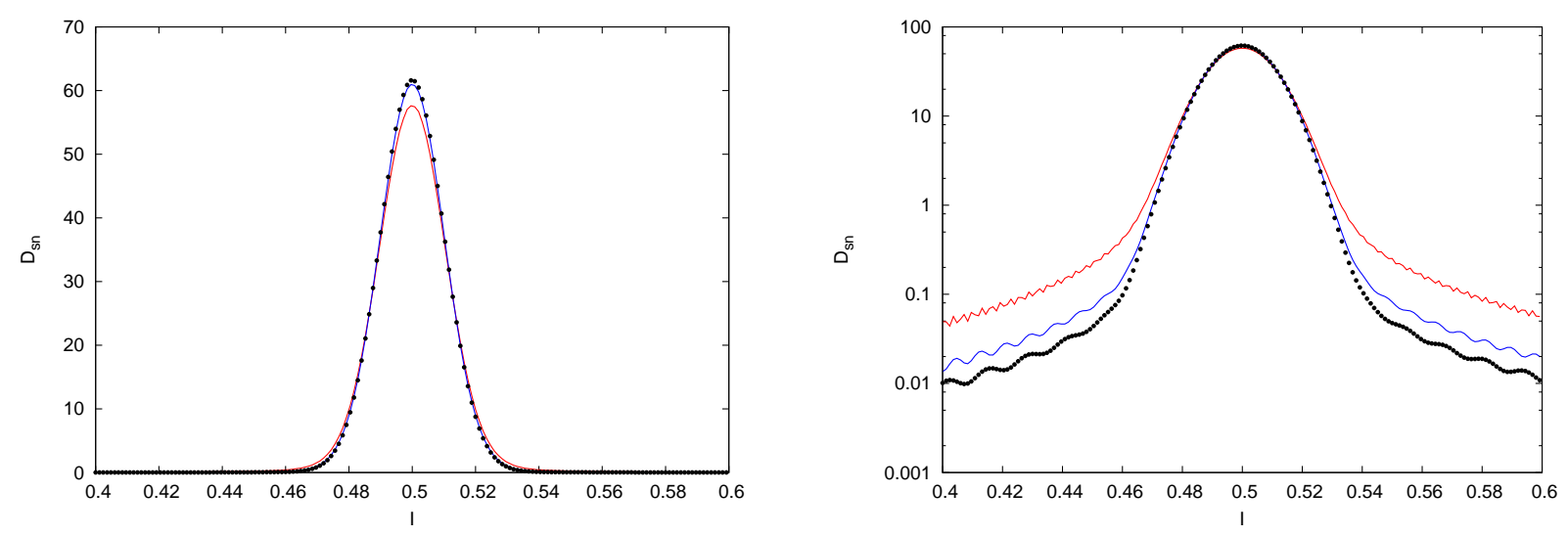

Figura 5.3: $\mathcal{D}_{s n}(I ; N)$, para $N=10^{3}, 3 \times 10^{3}, 5 \times 10^{3}$, respectivamente en colores rojo, azul y negro, utilizando $\omega_{0}=0.5, \eta=50$ y $\theta_{0} \approx 0.214$. A la derecha en escala semi-logarítmica.

literatura (Uhlenbeck y Ornstein 1930, Gillespie 1996b, entre otros).

Utilizando la terminología de Gillespie (1996a) y Gillespie (1996b), se tiene que el proceso de O-U puede ser descripto a través de la siguiente ecuación diferencial estocástica ${ }^{2}$ :

$$
\frac{d \xi_{t}}{d t}=-\frac{1}{\eta} \xi_{t}+c^{1 / 2} \frac{d W_{t}}{d t}
$$

donde $W_{t}$ es un movimiento Browniano (sección 1.3.2), con tiempo de correlación $\eta$, y coeficiente de difusión c. No debe confundirse este coeficiente de difusión del ruido (O-U), dado como parámetro, con el que se desea computar, que es el correspondiente a la quasi-acción $I$. Es posible demostrar que, para una condición inicial puntual $\xi_{0}$, la densidad de probabilidad del proceso O-U se escribe en términos de la función normal (1.63) así:

$$
\rho(\xi, t)=\mathcal{N}\left(\xi ; \xi_{0} e^{-\left(t-t_{0}\right) / \eta}, \frac{c \eta}{2}\left(1-e^{-2\left(t-t_{0}\right) / \eta}\right)\right) \quad\left(t \geq t_{0}\right) .
$$

Se dice que el proceso O-U es estable porque existe el límite

$$
\rho_{*}(\xi)=\lim _{t_{0} \rightarrow-\infty} \rho(\xi, t)=\mathcal{N}(\xi ; 0, \eta c / 2),
$$

que resulta ser independiente de ambos $\xi_{0}$ y $t$. Esta función es llamada distribución estacionaria del mencionado proceso mientras que la función de autocorrelación correspondiente es:

$$
\phi(t, s)=\sigma_{\xi}^{2} e^{-|s-t| / \eta} ;
$$

donde $\sigma_{\xi}^{2}=\eta c / 2$ es la varianza de la distribución del proceso $\mathrm{O}-\mathrm{U}$.

Aplicando la transformada de Fourier a la función de autocorrelación se llega a:

$$
\tilde{\phi}(\nu)=\sigma_{\xi}^{2}\left\{\frac{1}{1-e^{\left(-\frac{1}{\eta}+i \nu\right)}}+\frac{e^{\left(-\frac{1}{\eta}-i \nu\right)}}{1-e^{\left(-\frac{1}{\eta}-i \nu\right)}}\right\},
$$

\footnotetext{
${ }^{2}$ Las definiciones de integral estocástica y de ecuación diferencial estocástica pueden ser consultadas en Arnold (1973).
} 
y el coeficiente analítico resulta ser la siguiente función:

$$
\mathcal{D}(I)=\frac{\sigma_{\xi}^{2}}{4}\left\{\frac{1}{1-e^{\left(-\frac{1}{\eta}-i I\right)}}+\frac{e^{\left(-\frac{1}{\eta}+i I\right)}}{1-e^{\left(-\frac{1}{\eta}+i I\right)}}+\frac{1}{1-e^{\left(-\frac{1}{\eta}+i I\right)}}+\frac{e^{\left(-\frac{1}{\eta}-i I\right)}}{1-e^{\left(-\frac{1}{\eta}-i I\right)}}\right\}
$$

La función de autocorrelación y el coeficiente de difusión analítico son ambas funciones reales ya que las correspondientes partes imaginarias son nulas.

En la Fig. 5.4-izq. se comparan entre sí la predicción analítica, $\mathcal{D}_{a}(I)$ (rojo) y la medición numérica, $\mathcal{D}_{n u}\left(I ; 10^{3}\right)$ (azul), para ruido de O-U utilizando $\eta=50$ y $\theta_{0} \approx 0.214$, en el intervalo $0 \leq I \leq 7$. El coeficiente numérico se computó utilizando la fórmula (5.24) con $\epsilon=10^{-6} \mathrm{y}$ promediando sobre $N_{r}=2 \times 10^{3}$ realizaciones del ruido. Para generar este ruido se utilizó el algoritmo desarrollado en Fox et al. (1988). Se observa un acuerdo entre ambas formas de computar el coeficiente de difusión, salvo para $I \approx 0$. En la Fig. 5.4-der. se ha agregado a la comparación el coeficiente semi-numérico, $\mathcal{D}_{s n}^{\star}\left(I ; 10^{4}\right)$ (negro), focalizando en el intervalo $0 \leq I \leq 0.05$, utilizando $N_{r}=10^{4}$. Aquí se puede apreciar que en el entorno de $I=0$, ambas predicciones $\mathcal{D}_{s n}^{\star}$ y $\mathcal{D}_{a}$ difieren de la medición $\mathcal{D}_{n u}$. Lo mismo sucede para el resto de los valores de la forma $I=2 \pi q, q \in \mathbb{Z}$, que son justamente aquellos donde la validez del teorema no esta garantizada.
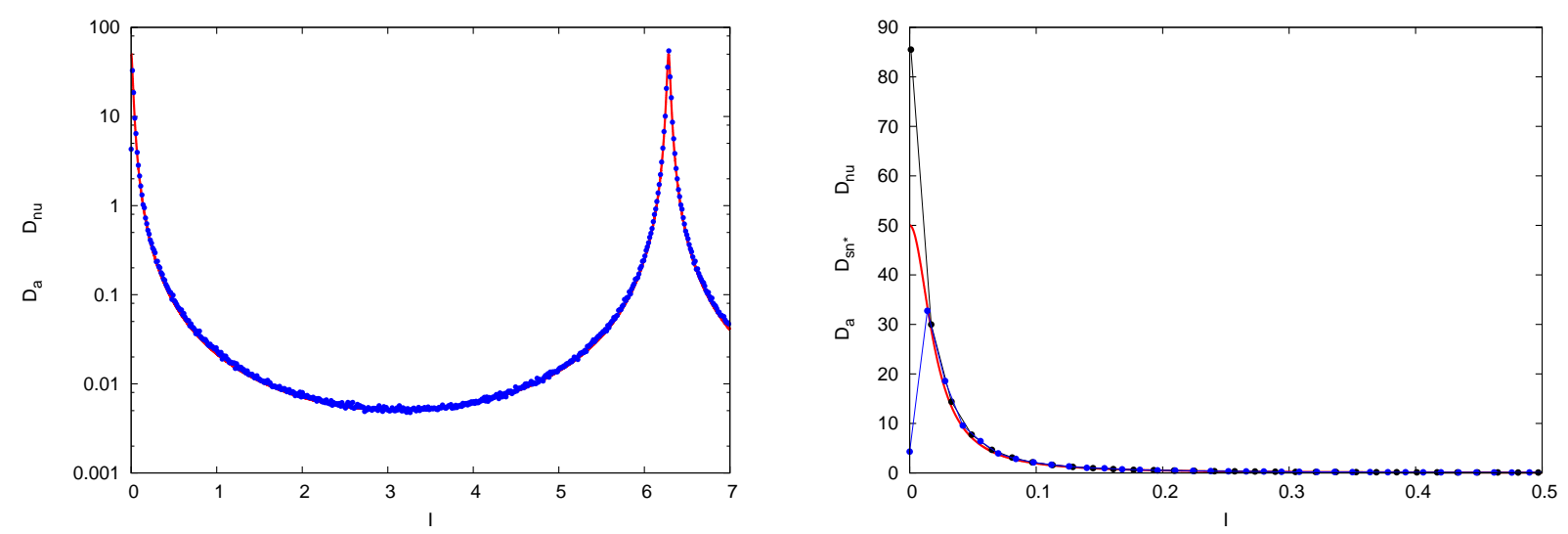

Figura 5.4: A la izquierda se comparan entre sí la predicción analítica, $\mathcal{D}_{a}(I)$ (rojo) y la medición numérica, $\mathcal{D}_{n u}\left(I ; 10^{3}\right)$ (azul), para ruido de O-U utilizando $\eta=50$ y $\theta_{0} \approx 0.214$, en el intervalo $0 \leq I \leq 7$. El coeficiente numérico se computó utilizando formula (5.24) con $\epsilon=10^{-6}$ y promediando sobre $N_{r}=2 \times 10^{3}$ realizaciones del ruido. A la derecha se ha agregado a la comparación el coeficiente semi-numérico, $\mathcal{D}_{s n}^{\star}\left(I ; 10^{4}\right)$ (negro), focalizando en el intervalo $0 \leq I \leq 0.05$, utilizando $N_{r}=10^{4}$.

\subsubsection{Ecuación de Langevin de un oscilador armónico amortiguado}

La ecuación de Langevin para la posición $\xi_{t}$ de una partícula Browniana de masa unitaria sujeta a un campo de fuerzas de un oscilador ideal es:

$$
\frac{d^{2} \xi_{t}}{d t^{2}}=-\lambda \frac{d \xi_{t}}{d t}-\omega^{2} \xi_{t}+c^{1 / 2} \frac{d W_{t}}{d t}
$$


donde $\omega$ es la frecuencia determinista del oscilador no perturbado, $\lambda$ es el coeficiente de fricción y $c$ se relaciona con el coeficiente de difusión, $D_{W}$, que tendría la partícula Browniana en caso de potencial nulo $(\omega=0)$, así:

$$
D_{W}=c / 2 \lambda^{2} .
$$

Introduciendo el momento de la partícula $p=\dot{\xi}$ se reescribe a la Ec. (5.41) como un sistema de ecuaciones diferenciales estocásticas de primer orden:

$$
d\left(\begin{array}{c}
p_{t} \\
\xi_{t}
\end{array}\right)=\left(\begin{array}{cc}
-\lambda & -\omega^{2} \\
1 & 0
\end{array}\right)\left(\begin{array}{c}
p_{t} \\
\xi_{t}
\end{array}\right) d t+\left(\begin{array}{c}
c^{1 / 2} \\
0
\end{array}\right) d W_{t}
$$

Para valores de parámetros $\omega=1, \lambda=0.2$ y $c=0.4$ se ha realizado una integración ${ }^{3}$ numérica de esta ecuación con un esquema de Euler-Maruyama (Higham, 2001), obteniendo una función de autocorrelación que puede ser ajustada, aproximadamente, como:

$$
\phi(\tau)=a \cos \left(\omega_{0} \tau\right) e^{-b \tau} ;
$$

con valores $a=1.051 \approx 1, b=0.096 \approx 0.1 \mathrm{y} \omega_{0}=0.980 \approx 1$.

La densidad espectral correspondiente está dada por la siguiente función real:

$$
\tilde{\phi}(\nu)=\frac{a}{2}\left\{\frac{1}{1-e^{\left(i\left(\nu+\omega_{0}\right)-b\right)}}+\frac{1}{1-e^{\left(i\left(\nu-\omega_{0}\right)-b\right)}}+\frac{e^{-\left(i\left(\nu+\omega_{0}\right)+b\right)}}{1-e^{-\left(i\left(\nu+\omega_{0}\right)+b\right)}}+\frac{e^{-\left(i\left(\nu-\omega_{0}\right)+b\right)}}{1-e^{-\left(i\left(\nu-\omega_{0}\right)+b\right)}}\right\} .
$$

Utilizando las Ecs. (5.44) y (5.17) se ha computado $\mathcal{D}_{s n}\left(I ; 10^{3}\right)$ con $\theta_{0}=\sqrt{5} / 2 \approx 1.118$, mientras que utilizando las Ecs. (5.45) y (5.22) se ha computado $\mathcal{D}_{a}(I)$. Ambas curvas se presentan en la Fig. 5.7, donde se aprecia un buen acuerdo entre ellas.
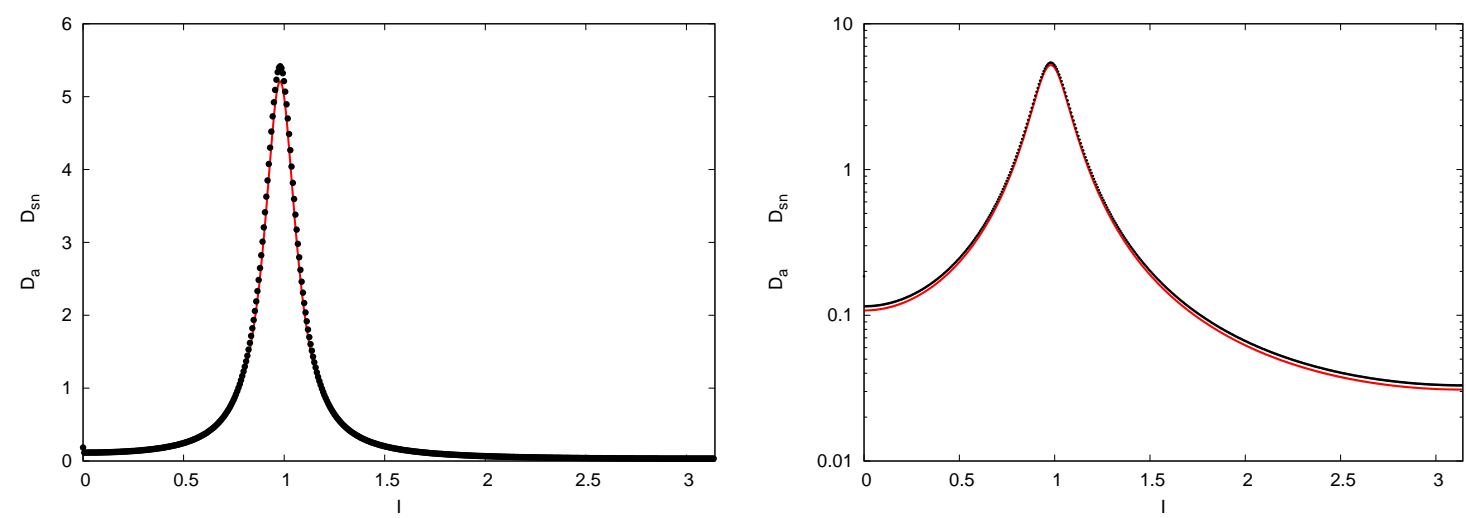

Figura 5.5: $\mathcal{D}_{a}(I)$ (rojo) y $\mathcal{D}_{s n}\left(I ; 10^{3}\right)$ (negro) en escalas natural (izquierda) y semi-logarítmica (derecha). Los parámetros utilizados fueron $\omega=1, \lambda=0.2$ y $c=0.4$. Además, para $\mathcal{D}_{s n}$ se utilizó $\theta_{0}=\sqrt{5} / 2$.

Luego, se ha computado el coeficiente numérico, $\mathcal{D}_{n u}\left(I ; 10^{3}\right)$, utilizando $N_{r}=10^{4}$ realizaciones del ruido, $\epsilon=10^{-7}$ y $\theta_{0} \approx 0.214$. En la Fig. 5.8 se compara dicha cantidad con

\footnotetext{
${ }^{3}$ Dado que la ecuación diferencial estocástica (5.43) es lineal y que en la misma el término proporcional a $d W$ es una constante, y por lo tanto una función determinista, no fue necesario adoptar una de las (infinitas) interpretaciones de la integral estocástica (por ejemplo: Itô, Stratonovich; ver Stratonovich and Silverman (1967)). Para esta situación todas devienen en la misma cantidad.
} 
su contrapartida analítica. Puede apreciarse que en ciertas regiones hay concordancia entre ambos coeficientes, mientras que en otras no. Por ejemplo, cerca de $I \approx 1$, que corresponde al maximo absoluto, se obtiene un error relativo de aproximadamente el $30 \%$. Este máximo corresponde con la resonancia $(1,1)$ entre el mapa integrable y el ruido. Se realizaron otros experimentos numéricos variando $\epsilon$. Se concluyó que siempre que se mantenga dicho parámetro por debajo de $10^{-3}$, no se aprecian diferencias entre los coficientes numéricos normalizados (reescaleados).
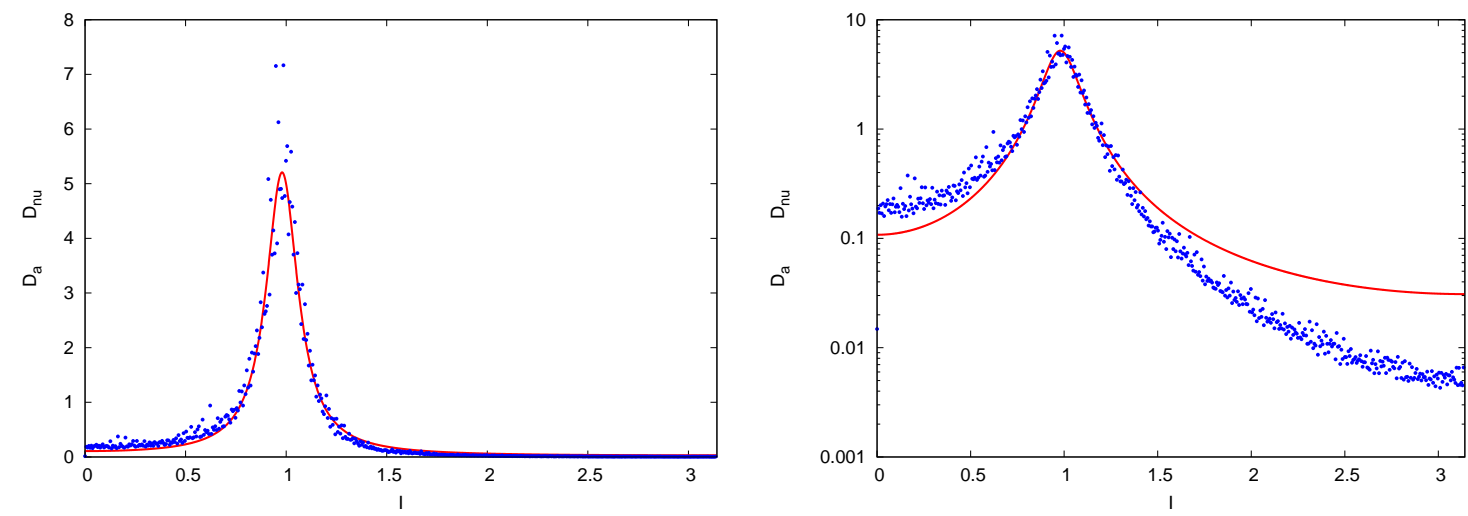

Figura 5.6: y $\mathcal{D}_{a}(I)$ (rojo) $\left.\mathcal{D}_{n u}\left(I ; 10^{3}\right)\right)$ (azul) en escalas natural (izquierda) y semi-logarítmica (derecha). Los parámetros usados son $N_{r}=10^{4}, \omega=1, \lambda=0.2$ y $c=0.4$. Además, para $\mathcal{D}_{n u}$ se utilizó $\theta_{0} \approx 0.214$.

La ecuación de Langevin para la posición $\xi_{t}$ de una partícula Browniana de masa unitaria sujeta a un campo de fuerzas de un oscilador ideal es:

$$
\frac{d^{2} \xi_{t}}{d t^{2}}=-\lambda \frac{d \xi_{t}}{d t}-\omega^{2} \xi_{t}+c^{1 / 2} \frac{d W_{t}}{d t}
$$

donde $\omega$ es la frecuencia determinista del oscilador no perturbado, $\lambda$ es el coeficiente de fricción y $c$ se relaciona con el coeficiente de difusión, $D_{W}$, que tendría la partícula Browniana en caso de potencial nulo $(\omega=0)$, así:

$$
D_{W}=c / 2 \lambda^{2} .
$$

Introduciendo el momento de la partícula $p=\dot{\xi}$ se reescribe a la Ec. (5.41) como un sistema de ecuaciones diferenciales estocásticas de primer orden:

$$
d\left(\begin{array}{c}
p_{t} \\
\xi_{t}
\end{array}\right)=\left(\begin{array}{cc}
-\lambda & -\omega^{2} \\
1 & 0
\end{array}\right)\left(\begin{array}{c}
p_{t} \\
\xi_{t}
\end{array}\right) d t+\left(\begin{array}{c}
c^{1 / 2} \\
0
\end{array}\right) d W_{t}
$$

Para valores de parámetros $\omega=1, \lambda=0.2$ y $c=0.4$ se ha realizado una integración ${ }^{4}$ numérica de esta ecuación con un esquema de Euler-Maruyama (Higham, 2001), obteniendo una función de autocorrelación que puede ser ajustada, aproximadamente, como:

$$
\phi(\tau)=a \cos \left(\omega_{0} \tau\right) e^{-b \tau} ;
$$

\footnotetext{
${ }^{4}$ Dado que la ecuación diferencial estocástica (5.43) es lineal y que en la misma el término proporcional a $d W$ es una constante, y por lo tanto una función determinista, no fue necesario adoptar una de las (infinitas) interpretaciones de la integral estocástica (por ejemplo: Itô, Stratonovich; ver Stratonovich and Silverman (1967)). Para esta situación todas devienen en la misma cantidad.
} 
con valores $a=1.051 \approx 1, b=0.096 \approx 0.1 \mathrm{y} \omega_{0}=0.980 \approx 1$.

La densidad espectral correspondiente está dada por la siguiente función real:

$$
\tilde{\phi}(\nu)=\frac{a}{2}\left\{\frac{1}{1-e^{\left(i\left(\nu+\omega_{0}\right)-b\right)}}+\frac{1}{1-e^{\left(i\left(\nu-\omega_{0}\right)-b\right)}}+\frac{e^{-\left(i\left(\nu+\omega_{0}\right)+b\right)}}{1-e^{-\left(i\left(\nu+\omega_{0}\right)+b\right)}}+\frac{e^{-\left(i\left(\nu-\omega_{0}\right)+b\right)}}{1-e^{-\left(i\left(\nu-\omega_{0}\right)+b\right)}}\right\} .
$$

Utilizando las Ecs. (5.44) y (5.17) se ha computado $\mathcal{D}_{s n}\left(I ; 10^{3}\right)$ con $\theta_{0}=\sqrt{5} / 2 \approx 1.118$, mientras que utilizando las Ecs. (5.45) y (5.22) se ha computado $\mathcal{D}_{a}(I)$. Ambas curvas se presentan en la Fig. 5.7, donde se aprecia un buen acuerdo entre ellas.
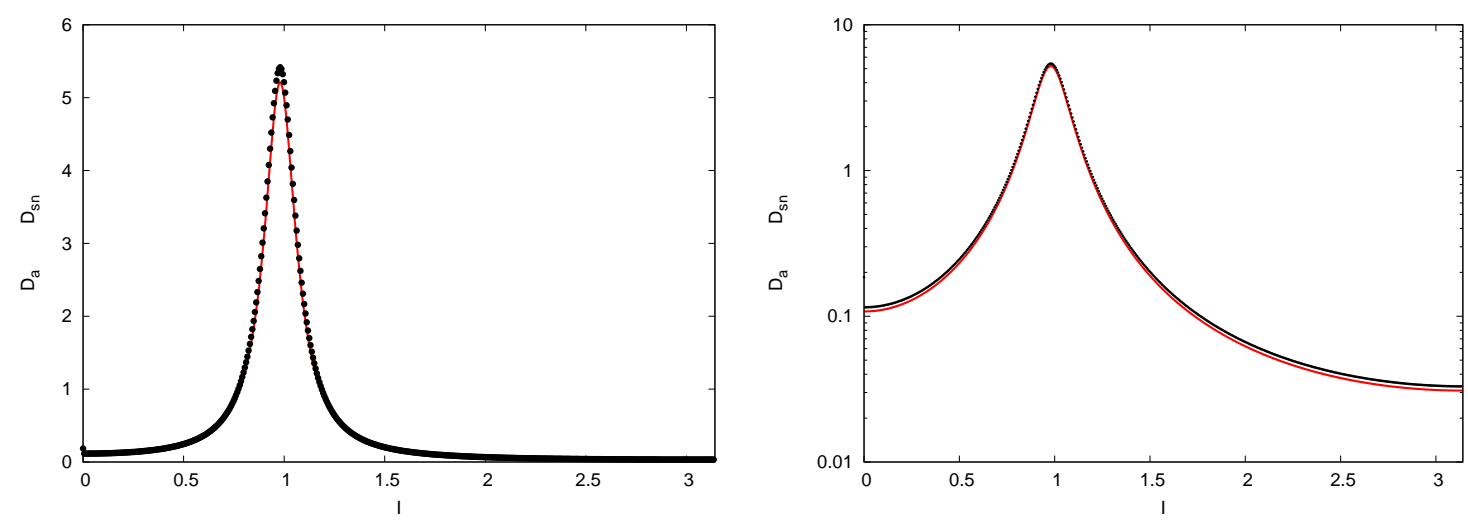

Figura 5.7: $\mathcal{D}_{a}(I)$ (rojo) y $\mathcal{D}_{s n}\left(I ; 10^{3}\right)$ (negro) en escalas natural (izquierda) y semi-logarítmica (derecha). Los parámetros utilizados fueron $\omega=1, \lambda=0.2$ y $c=0.4$. Además, para $\mathcal{D}_{s n}$ se utilizó $\theta_{0}=\sqrt{5} / 2$.

Luego, se ha computado el coeficiente numérico, $\mathcal{D}_{n u}\left(I ; 10^{3}\right)$, utilizando $N_{r}=10^{4}$ realizaciones del ruido, $\epsilon=10^{-7}$ y $\theta_{0} \approx 0.214$. En la Fig. 5.8 se compara dicha cantidad con su contrapartida analítica. Puede apreciarse que en ciertas regiones hay concordancia entre ambos coeficientes, mientras que en otras no. Por ejemplo, cerca de $I \approx 1$, que corresponde al maximo absoluto, se obtiene un error relativo de aproximadamente el $30 \%$. Este máximo corresponde con la resonancia $(1,1)$ entre el mapa integrable y el ruido. Se realizaron otros experimentos numéricos variando $\epsilon$. Se concluyó que siempre que se mantenga dicho parámetro por debajo de $10^{-3}$, no se aprecian diferencias entre los coficientes numéricos normalizados (reescaleados).

\subsection{Perturbaciones deterministas}

A lo largo de este capítulo se ha podido entender que la función de autocorrelación del ruido tiene un efecto importante en la difusión. De hecho, la expresión para $\mathcal{D}(I)$ depende separadamente de las órbitas de la parte integrable y de la función de autocorrelación de la perturbación o ruido.

Así, es posible preguntarnos si las hipótesis bajo las cuales es válido el teorema para sistemas estocásticos, cuyo resultado es la ec. de F-P (5.9) con coeficiente de difusión dado por la Ec. (5.13), podrían ser modificadas para admitir una perturbación determinista. Se mostrará empíricamente que una perturbación caótica produce una difusión en la parte integrable que 

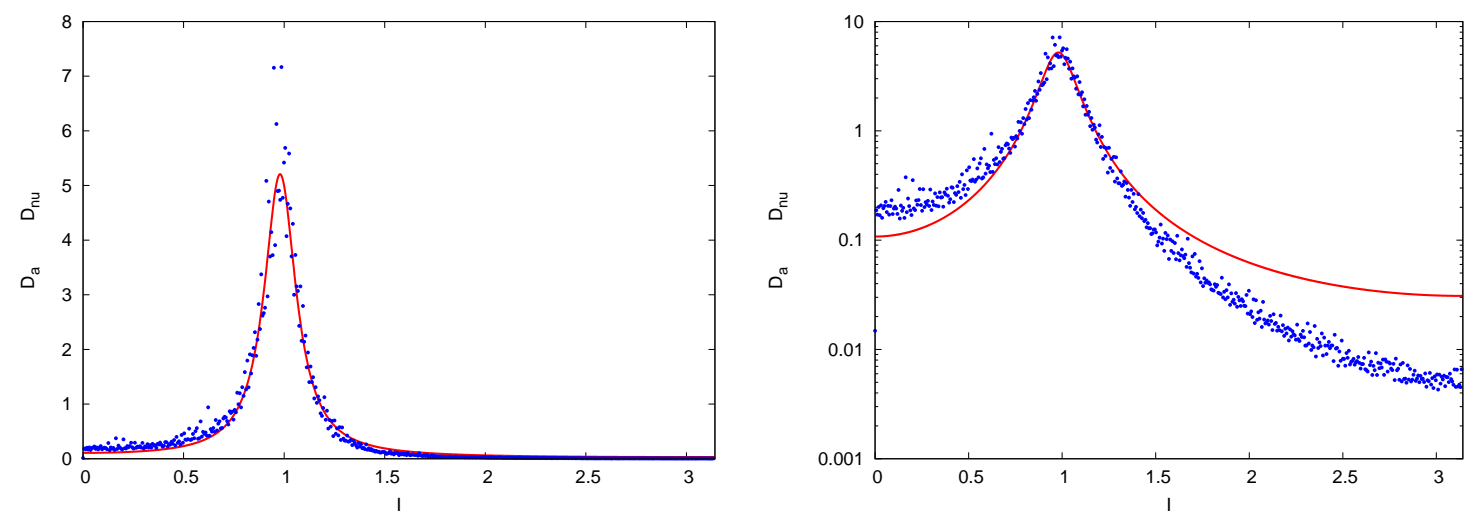

Figura 5.8: y $\mathcal{D}_{a}(I)$ (rojo) $\left.\mathcal{D}_{n u}\left(I ; 10^{3}\right)\right)$ (azul) en escalas natural (izquierda) y semi-logarítmica (derecha). Los parámetros usados son $N_{r}=10^{4}, \omega=1, \lambda=0.2$ y $c=0.4$. Además, para $\mathcal{D}_{n u}$ se utilizó $\theta_{0} \approx 0.214$.

es descriptible por dicha ecuación cinética. La única diferencia radicará en la fórmula para el coeficiente de difusión.

Luego de introducir dos mapas intermedios se definirá un mapa simpléctico 4D cuyas propiedades difusivas estudiaremos durante el resto de este capítulo. Además, para estos tres mapas se computará $\mathcal{D}_{n u}$. Posteriormente se realizarán cambios necesarios en las fórmulas de predicción de los coeficientes de difusión.

\subsubsection{Del formalismo estocástico a la simplecticidad}

Se inicia considerando el mapa dado por (5.15), donde ahora $\xi_{n}$ es el valor al tiempo $n$ de alguna variable dinámica determinísticamente obtenida. Se desea observar qué le sucede al coeficiente numérico cuando $\xi_{n}=\sin \left(\psi_{n}\right)$, donde $\psi_{n}$ es el ángulo al tiempo $n$ correspondiente a alguna trayectoria caótica de un SM:

$$
\begin{aligned}
& J_{n+1}=J_{n}+K \sin \left(\psi_{n}\right) \\
& \psi_{n+1}=\psi_{n}+J_{n+1} \quad \bmod 2 \pi \text {. }
\end{aligned}
$$

El concepto de realización de un proceso aleatorio, es así, reinterpretado, utilizando órbitas caóticas del $S M$. Ahora, el espacio de muestras (sección 1.3.2) es algún subconjunto de trayectorias pertenecientes a la capa estocástica del SM. Debido a la propiedad de unicidad de trayectorias deterministas, es posible etiquetar unívocamente a $\mathcal{M}$ con los valores de las condiciones iniciales en el plano $[J, \psi]$. Por ejemplo, en esta sección y en la siguiente se trabajará con un SM de parámetro $K=3, \mathrm{y}$

$$
\mathcal{M} \equiv\left\{\left(J_{0}, \psi_{0}\right): \quad J_{0}=3, \quad 0 \leq \psi_{0} \leq 2 \pi\right\}
$$

Para este valor de $K$, el espacio de fases consiste en un mar caótico generalizado excepto por dos islas de estabilidad (enteras), que son relativamente pequeñas y están centradas en puntos de la forma $(J, \psi) \approx(2 k \pi, \pi)(k \in \mathbb{Z})$. La elección de $\mathcal{M}$ evita los centros de tales islas de regularidad, por lo que consiste fundamentalmente de órbitas caóticas. Esto hace que 
estas órbitas, si bien deterministas, tengan ciertas características similares a realizaciones de ruidos ${ }^{5}$.

El sistema completo de ecuaciones parcialmente acopladas resulta ser:

$$
\begin{array}{rlrl}
I_{n+1} & =I_{n}-\epsilon \sin \left(\psi_{n}\right) \sin \left(\theta_{n}\right) & \\
J_{n+1} & =J_{n}+K \sin \left(\psi_{n}\right) & \\
\theta_{n+1} & =\theta_{n}+I_{n+1} & & \bmod 2 \pi \\
\psi_{n+1} & =\psi_{n}+J_{n+1} & & \bmod 2 \pi .
\end{array}
$$

Se ha iterado este mapa para $\epsilon=10^{-7}, N_{r}=2 \times 10^{4}, \theta_{0} \approx 0.2143$ y $0 \leq I \leq 2 \pi$, computando $\mathcal{D}_{n u}\left(I ; 10^{3}\right)$. El resultado se muestra en la Fig. 5.9, donde se observan dos máximos absolutos en $I \approx \frac{\pi}{2}$ y en $I \approx \frac{3 \pi}{2}$ y dos máximos relativos en $I \approx 0$ y $I \approx \pi$.

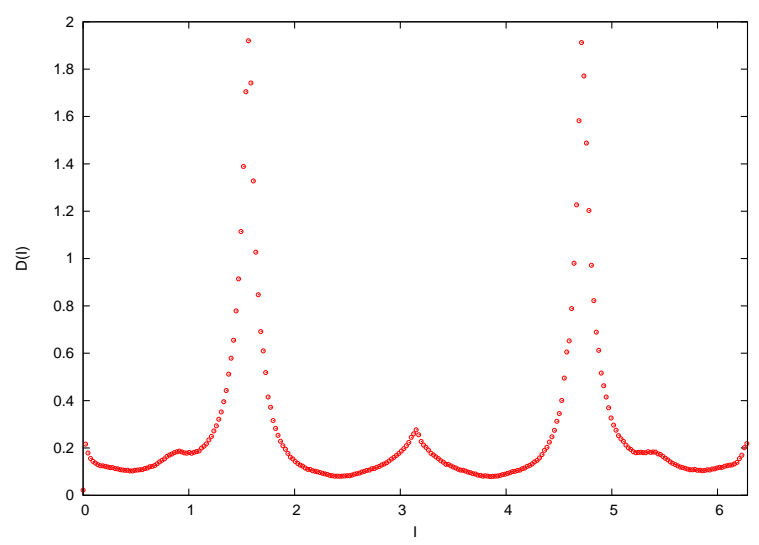

Figura 5.9: $\mathcal{D}_{n u}\left(I ; 10^{3}\right)$ para $\epsilon=10^{-7}, N_{r}=2 \times 10^{4}$ y $\theta_{0} \approx 0.2143$ para Ec. (5.48).

Como segundo modelo intermedio tenemos el siguiente mapa parcialmente acoplado:

$$
\begin{array}{rlrl}
I_{n+1} & =I_{n}-\epsilon \sin \left(\theta_{n}+\psi_{n}\right) & \\
J_{n+1} & =J_{n}+K \sin \left(\psi_{n}\right) & \\
\theta_{n+1} & =\theta_{n}+I_{n+1} & & \bmod 2 \pi \\
\psi_{n+1} & =\psi_{n}+J_{n+1} & & \bmod 2 \pi .
\end{array}
$$

Se realizó el cómputo de $\mathcal{D}_{n u}\left(I ; 10^{3}\right)$ utilizando los mismos parámetros que en caso anterior y dicho resultado se despliega en la Fig. 5.10, utilizando puntos rojos. Se puede apreciar una gran diferencia de los valores del coeficiente, respecto del mapa anterior, en el entorno de $I \approx \pi$.

La generalización final consiste en el mapa simpléctico 4D que nos interesa estudiar:

$$
\begin{aligned}
& I_{n+1}=I_{n}-\epsilon \sin \left(\theta_{n}+\psi_{n}\right) \\
& J_{n+1}=J_{n}+K \sin \left(\psi_{n}\right)-\epsilon \sin \left(\theta_{n}+\psi_{n}\right) \\
& \theta_{n+1}=\theta_{n}+I_{n+1} \quad \bmod 2 \pi \\
& \psi_{n+1}=\psi_{n}+J_{n+1} \quad \bmod 2 \pi ;
\end{aligned}
$$

\footnotetext{
${ }^{5} \mathrm{Al}$ utilizar un generador de números aleatorios se está, en el fondo, aprovechando justamente esta propiedad. Es decir, se utiliza un mapa ergódico determinista (no necesariamente caótico) para emular realizaciones de un proceso estocástico.
} 
que es generado por el Hamiltoniano dependiente del tiempo:

$$
H(I, J, \theta, \psi, t)=\frac{1}{2}\left(I^{2}+J^{2}\right)+\{K \cos (\psi)-\epsilon \cos (\theta+\psi)\} \sum_{n=-\infty}^{\infty} \delta(t-n),
$$

y puede ser interpretado com un rotador libre acoplado con un SM. Es el mismo mapa que fue utilizado por Kook y Meiss (1990) al estudiar el método de pumping estocástico.

Utilizando nuevamente los mismos parámetros de iteración, se ha computado $\mathcal{D}_{n u}\left(I ; 10^{3}\right)$ y se despliega en la Fig. 5.10 utilizando puntos verdes. Dicha figura muestra la similitud entre los coeficientes numéricos de difusión de los mapas (5.49) y (5.50). Esto no es sorprendente por la siguiente razón. Ya es bien conocido que las propiedades estadísticas de mapas ergódicos son estables ante perturbaciones suficientemente pequeñas. Las variables caóticas $(J, \psi)$ no pertenecen estrictamente a un sistema ergódico pero poseen aproximadamente esta cualidad en el mar caótico (capa estocástica gruesa). Luego, como la diferencia entre el mapa parcialmente acoplado (5.49) y el mapa simpléctico (5.50) es sólo una perturbación de $\mathcal{O}(\epsilon)$ a la parte "ergódica", se tiene la plausibilidad en la similitud entre ambos coeficientes de difusión.

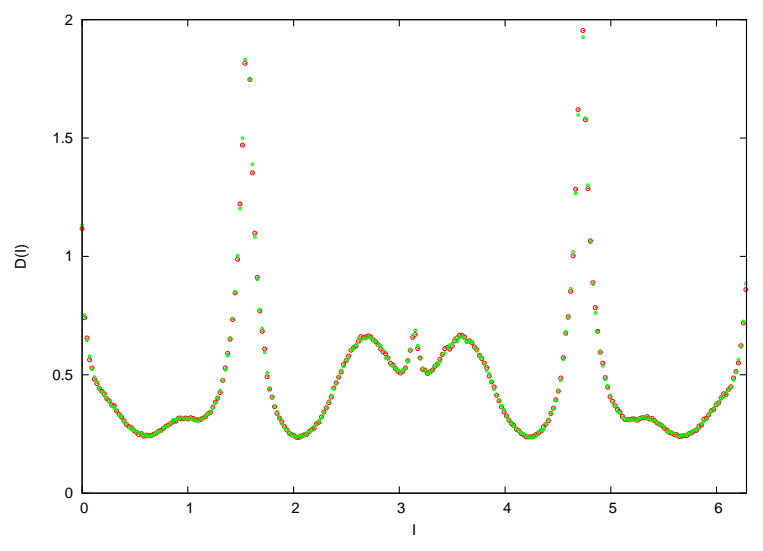

Figura 5.10: $\mathcal{D}_{n u}\left(I ; 10^{3}\right)$ para ambos mapas (5.49) y (5.50), usando $\epsilon=10^{-7}, N_{r}=2 \times 10^{4} \mathrm{y}$ $\theta_{0} \approx 0.2143$.

\subsubsection{Generalización del método}

Anteriormente se observó la igualdad entre los coeficientes numéricos del mapa simpléctico (5.50) y del parcialmente acoplado (5.49). Se desea aplicar a éste último mapa, el método estocástico definido y aplicado en las secciones anteriores, con la intención de poder predecir el coeficiente $\mathcal{D}_{n u}$ correspondiente al mapa simpléctico. Para ello, antes es necesario generalizar las fórmulas teóricas para incluir una perturbación más general.

Entonces, suponiendo que en vez de la Ec. (5.16) se tiene una perturbación de la forma:

$$
H_{1}(I, \theta, \psi)=\sum_{k} h_{k}(I) e^{i k(\theta+\psi)}
$$

e introduciendo las funciones de autocorrelación armónico-dependientes:

$$
\phi_{k, k^{\prime}}(n, m)=E\left[e^{i k \psi_{n}} e^{i k^{\prime} \psi_{m}}\right],
$$


se tiene que el coeficiente de difusión semi-numérico (5.17) puede escribirse de la forma:

$$
\begin{aligned}
\mathcal{D}_{s n}(I ; N)= & \frac{1}{N} \sum_{n=0}^{N-1} \sum_{m=0}^{N-1} \sum_{k} \sum_{k^{\prime}} k k^{\prime} h_{k}(I) h_{k^{\prime}}(I) e^{i\left(k+k^{\prime}\right) \theta} e^{i\left(k \Omega(I) n+k^{\prime} \Omega(I) m\right)} \phi_{k, k^{\prime}}(n, m) \\
= & \sum_{k} \sum_{k^{\prime}} k k^{\prime} h_{k}(I) h_{k^{\prime}}(I) e^{i\left(k-k^{\prime}\right) \theta} \\
& \times\left\{\sum_{m=-N+1}^{N-1} \phi_{k, k^{\prime}}(|m|) e^{i k^{\prime} \Omega(I) m} \frac{1}{N} \sum_{n=j}^{j+N-1} e^{i\left(k-k^{\prime}\right) \Omega(I) n}\right\} .
\end{aligned}
$$

Usando estas ecuaciones junto con (5.7) y tomando en cuenta el hecho de que

$$
\lim _{N \rightarrow \infty} \frac{1}{N} \sum_{n=j}^{j+N-1} e^{i\left(k-k^{\prime}\right) \Omega(I) n}=\delta_{k, k^{\prime}}
$$

se expresa el coeficiente analítico así:

$$
\begin{aligned}
\mathcal{D}_{a}(I) & =\lim _{N \rightarrow \infty} \mathcal{D}_{s n}(I ; N) \\
& =\sum_{k}^{|k| \leq k_{\max }} k^{2} h_{k}^{2}(I) \sum_{m=-\infty}^{\infty} \phi_{k, k}(|m|) e^{i k \Omega(I) m} \\
& =\sum_{k}^{|k| \leq k_{\max }} k^{2} h_{k}^{2}(I) \tilde{\phi}_{k, k}(k \Omega(I)), \quad \forall I \in \mathcal{U} .
\end{aligned}
$$

Comparando este resultado con Ec. (5.13) se tiene que en este enfoque más general cada armónico de la perturbación contribuye con una función densidad espectral diferente.

Para nuestro sistema determinista el método semi-numérico puede ser reescrito utilizando funciones sinusoidales y consenoidales (en vez de exponenciales) así:

$$
\begin{aligned}
\mathcal{D}_{s n}(I ; N)=\frac{1}{N} \sum_{n=0}^{N-1} \sum_{m=0}^{N-1} & \left\{\phi_{1}(n, m) \cos (\theta+\Omega(I) n) \cos (\theta+\Omega(I) m)\right. \\
& +\phi_{2}(n, m) \sin (\theta+\Omega(I) n) \sin (\theta+\Omega(I) m) \\
& +\phi_{3}(n, m) \cos (\theta+\Omega(I) n) \sin (\theta+\Omega(I) m) \\
& \left.+\phi_{4}(n, m) \sin (\theta+\Omega(I) n) \cos (\theta+\Omega(I) m)\right\} ;
\end{aligned}
$$

donde

$$
\begin{aligned}
& \phi_{1}(n, m)=E\left[\sin \psi_{n} \sin \psi_{m}\right], \\
& \phi_{2}(n, m)=E\left[\cos \psi_{n} \cos \psi_{m}\right], \\
& \phi_{3}(n, m)=E\left[\sin \psi_{n} \cos \psi_{m}\right], \\
& \phi_{4}(n, m)=E\left[\cos \psi_{n} \sin \psi_{m}\right] .
\end{aligned}
$$

No se conoce expresión analítica, válida hasta tiempos del orden de $10^{3}$, de dichas funciones de autocorrelación para el SM. En Meiss et al. (1983) y Cary et al. (1981) se presentan 
aproximaciones válidas bajo ciertas condiciones y para tiempos $\lesssim 10$. Por lo tanto, a partir de ahora la predicción del coeficiente de difusión se realizará utilizando la otra versión del coeficiente semi-numérico:

$$
\begin{aligned}
\mathcal{D}_{s n}^{\star}(I ; N)=\frac{1}{N} \sum_{n=0}^{N-1} \sum_{m=0}^{N-1} & \left\{\left\langle\sin \psi_{n} \sin \psi_{m}\right\rangle \cos (\theta+\Omega(I) n) \cos (\theta+\Omega(I) m)\right. \\
& +\left\langle\cos \psi_{n} \cos \psi_{m}\right\rangle \sin (\theta+\Omega(I) n) \sin (\theta+\Omega(I) m) \\
& +\left\langle\sin \psi_{n} \cos \psi_{m}\right\rangle \cos (\theta+\Omega(I) n) \sin (\theta+\Omega(I) m) \\
& \left.+\left\langle\cos \psi_{n} \sin \psi_{m}\right\rangle \sin (\theta+\Omega(I) n) \cos (\theta+\Omega(I) m)\right\}
\end{aligned}
$$

\subsection{Capa estocástica gruesa}

\subsubsection{Funciones de correlación}

A partir de ahora sólo se trabajarán con funciones de autocorrelación numéricas. Por lo tanto, a modo de simplificar la notación, durante el resto del capítulo se utilizará la siguiente convención:

$$
\phi_{i}(\tau) \equiv \phi_{i}\left(t_{0}+\tau, t_{0}\right) \equiv\left\langle\xi_{t_{0}+\tau} \xi_{t_{0}}\right\rangle \quad \text { para } \quad \tau>\mathrm{t}_{0},
$$

al referirnos a las funciones de autocorrelación numéricas. La elección de un valor inicial $t_{0}$, para empezar a computar las autocorrelaciones, se debe a que el conjunto $\mathcal{M}$ no es invariante y por lo tanto se precisa un cierto tiempo para que las realizaciones se distribuyan en toda la región caótica, superando así el transitorio inicial. Eligiendo $t_{0} \geq 500$, se tiene que las funciones $\phi_{i}$ son prácticamente independientes del valor de $t_{0}$. Un procedimiento similar fue realizado en Bazzani and Beccaceci (1998).

A continuación se analizará la función de autocorrelación del SM, y se comparará con la del oscilador armónico forzado perturbado estocásticamente, que fue analizado en la subsección 5.2.4. Allí, se obtuvo que este sistema estocástico posee un perfil de $\phi(\tau)$ que obedece (5.44). Tanto la función numérica como el ajuste se muestran en la Fig. 5.11 en colores rojo y verde, respectivamente. Allí es posible apreciar que el decaimiento de la amplitud de la correlación es exponencial al igual que la del ajuste.

Análogamente, se computó $\phi_{1}(\tau)$, con $t_{0}=500$, para el SM, utilizando $N_{r}=2 \times 10^{4}$. Dichos valores se despliegan, en color rojo, en la Fig. 5.12, utilizando dos rangos temporales distintos. Un ajuste numérico de la forma funcional (5.44) arrojó los parámetros: $a=0.436$, $b=0.117$ y $\omega_{0}=1.550$. Los mismos se despliegan en la mencionada figura, utilizando curvas de color verde. De estos datos se evidencia que en el SM las correlaciones no decaen a cero para $\tau>>1$, como sí sucedía en el caso estocástico. A pesar de esta diferencia, existe una clara similitud entre ambas funciones de autocorrelación. Esta semejanza motivó la idea de aplicar el método estocástico a un mapa simpléctico y fue sugerida en Bazzani and Beccaceci (1998) pero nunca desarrollada hasta la fecha.

Posteriormente se ajustó una función levemente distinta. Las Figs. 5.13 y 5.14 muestran que una función de correlación que funciona mejor está dada por

$$
\phi(\tau)=\left(a_{0}+a_{1} e^{-b \tau}\right) \cos \left(\omega_{0} \tau\right) ;
$$

con parámetros ajustados: $a_{0}=0.014, a_{1}=0.442 b=0.143$ y $\omega_{0}=1.567$. Sin embargo, observando ambas imágenes de la Fig. 5.14 se aprecia que para ciertos valores de $\tau$, los valores 


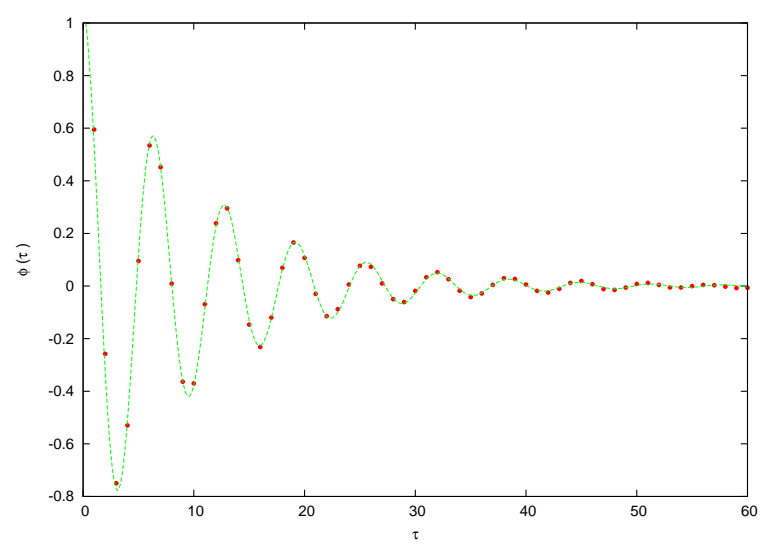

Figura 5.11: Gráfico de la función de correlación $\phi(\tau)$ (puntos) computada usando un ensamble de $10^{4}$ órbitas de ruido de Langevin con $\omega=1, \lambda=0.2$ y $c=0.4$. El ajuste según ansatz (5.44) se muestra en color verde.
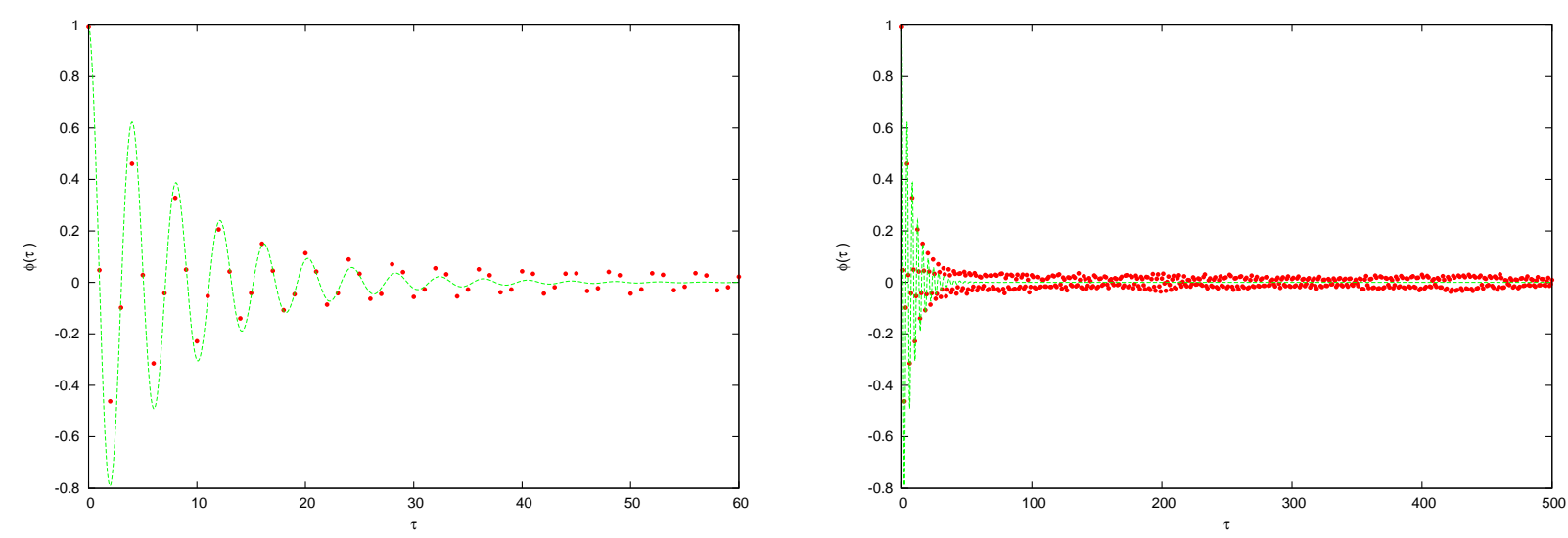

Figura 5.12: Gráfico de la función de correlación $\phi(\tau)$ (puntos) computada usando un ensamble de $2 \times 10^{4}$ órbitas del Mapa Estándar con condiciones iniciales uniformemente distribuídas en la variable angular en $J_{0}=3$. Los resultados numéricos están interpolados por una función $\cos \left(\omega_{0} \tau\right) e^{-b \tau}$ (curva) donde $b=0.117$ y $\omega_{0}=1.550$. Se evidencia que la función correlación no decae a cero.

numéricos de la autocorrelación se encuentra relativamente alejados del valor correspondiente al ansatz ajustado.

En la Fig. 5.15 se muestran estas cuatro funciones de autocorrelación, definidas en Ec. (5.58), para $\tau \in[0,500]$ y para $t_{0}=10^{3}$, en colores rojo, azul, verde y negro, respectivamente. Se puede observar que todas menos $\phi_{2}$ oscilan alrededor de cero. Además, se ve que $\phi_{1}$ presenta oscilaciones de mayor amplitud que el resto, mientras que $\phi_{3}$ y $\phi_{4}$ son en todo momento cercanas a cero. 


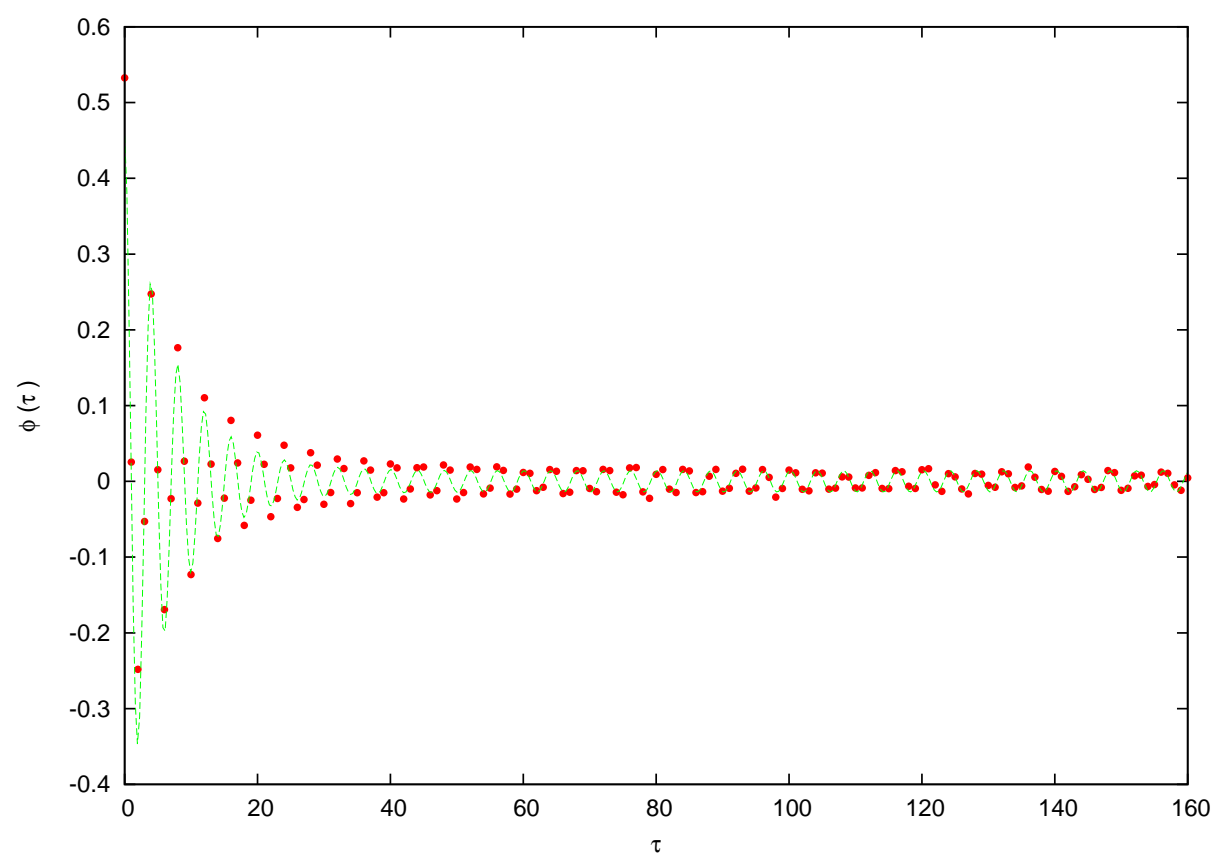

Figura 5.13: $\phi(\tau)$ (puntos) computada utilizando un ensamble de $2 \times 10^{4}$ órbitas del SM con condiciones iniciales uniformemente distribuídas en la variable angular en $J_{0}=3$. Los resultados numéricos están interpolados por la función (5.59) donde $a_{0}=0.014, a_{1}=0.442$, $\omega_{0}=1.567$ y $b=0.143$. Este ansatz funciona mejor.
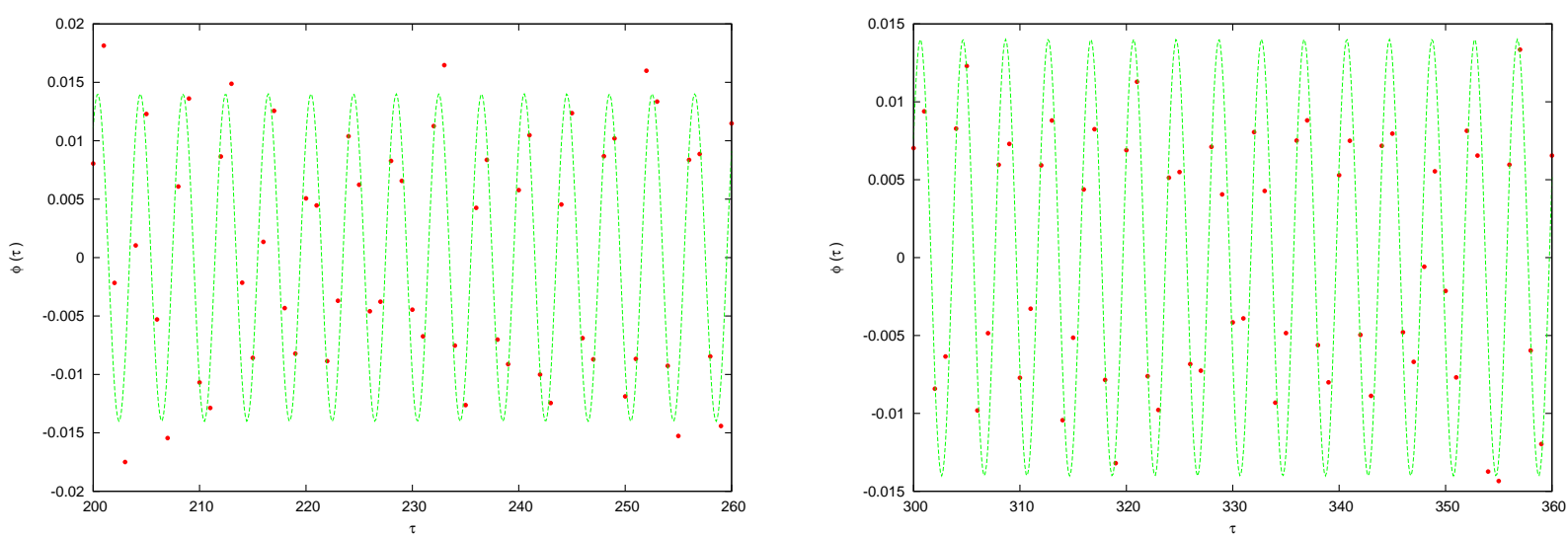

Figura 5.14: $\phi(\tau)$ (puntos) computada utilizando un ensamble de $2 \times 10^{4}$ órbitas del SM con condiciones iniciales uniformemente distribuídas en la variable angular en $J_{0}=3$. Los resultados numéricos están interpolados por la función (5.59) donde $a_{0}=0.014, a_{1}=0.442$, $\omega_{0}=1.567$ y $b=0.143$. Este ansatz funciona mejor.

\subsubsection{Coeficientes de difusión}

Con el objetivo de probar la fórmula (5.57) para $\mathcal{D}_{s n}^{\star}$, se ha leído de un archivo las cuatro funciones de autocorrelación computadas en la subsección anterior y se ha calculado 


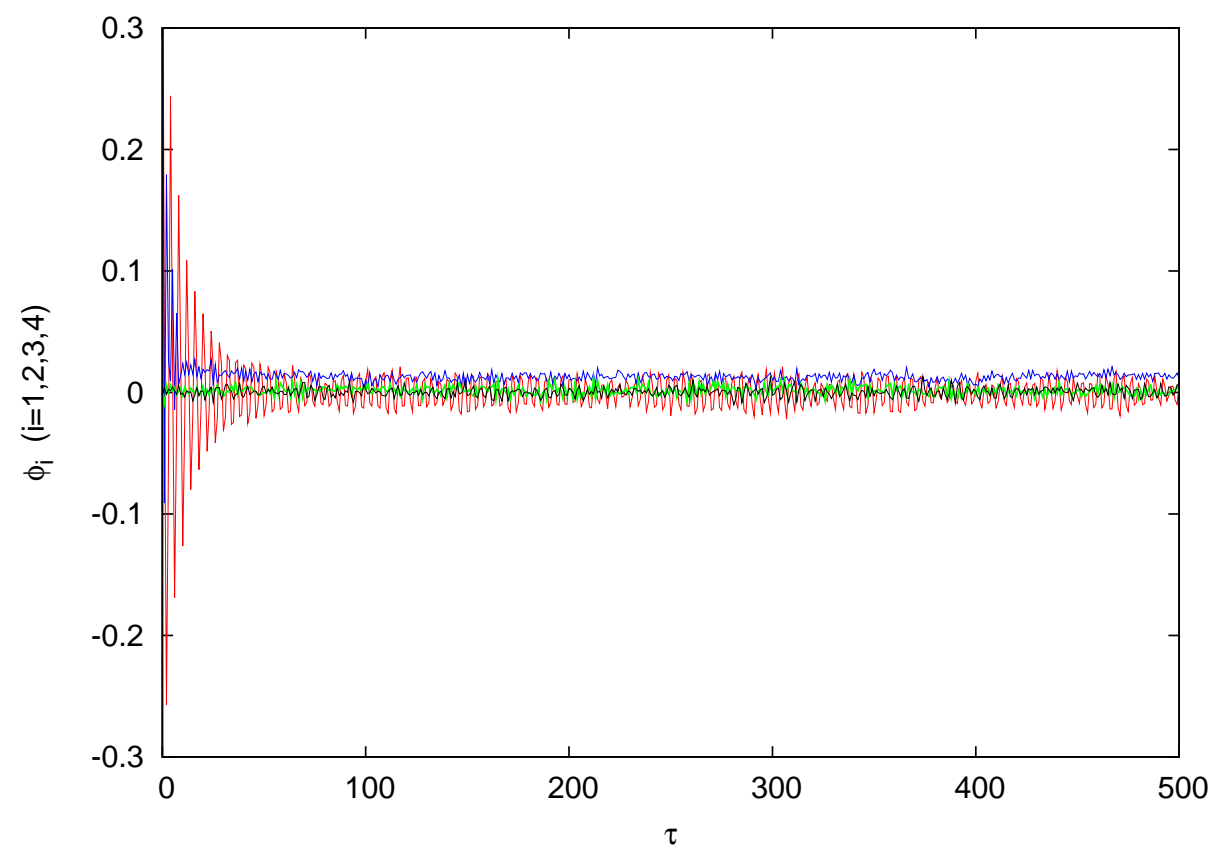

Figura 5.15: $\phi_{1}(\tau)$ (rojo), $\phi_{2}(\tau)$ (azul), $\phi_{3}(\tau)$ (verde) y $\phi_{4}(\tau)$ (negro), computadas utilizando un ensamble de $2 \times 10^{4}$ órbitas del $\mathrm{SM}(K=3)$ con condiciones iniciales uniformemente distribuídas en $\mathcal{M}$.

$\mathcal{D}_{s n}^{\star}\left(I ; 10^{3}\right)$, utilizando $\theta_{0} \approx 0.214$. En la Fig. 5.16 se compara este valor con la medición real, correspondiente al mapa (5.50) utilizando $K=3$ y $\epsilon=10^{-7}$. Se encuentra que $\mathcal{D}_{\text {sn }}^{\star}$ predice a $\mathcal{D}_{n u}$ con cierto detalle. Sin embargo, la predicción es aproximada ya que al movernos a lo largo del eje $I$, se observan oscilaciones respecto del valor medido. Se realizaron pruebas con otras combinaciones de valores de $N_{r}$ y $N$, pero no se pudo disminuir la amplitud de estas oscilaciones. Por lo tanto, si se desea utilizar este método para predecir el coeficiente de difusión, es necesario acompañar al método con algún proceso de suavizado de los datos.

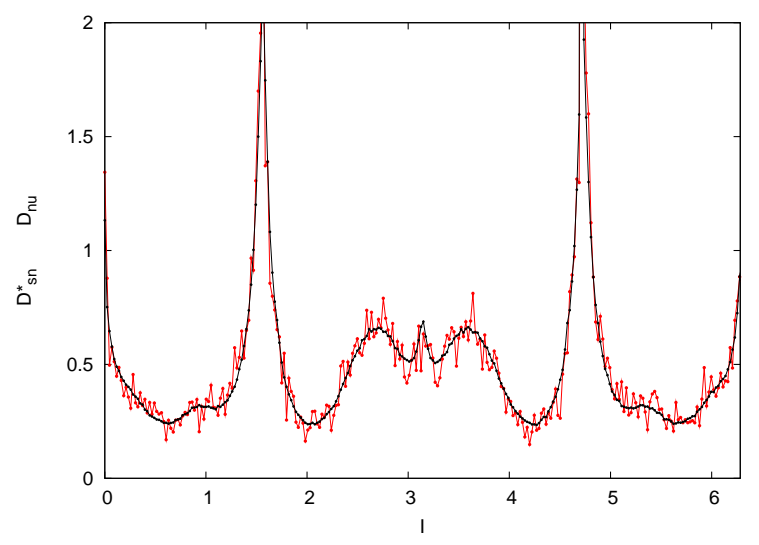

Figura 5.16: $\mathcal{D}_{s n}^{\star}\left(I ; 10^{3}\right)$ (rojo) y $\mathcal{D}_{n u}\left(I ; 10^{3}\right)$ (negro) para mapa simpléctico $(5.50)$, utilizando $K=3$ у $\epsilon=10^{-7}$. 


\subsubsection{La densidad de probabilidad}

A continuación se mostrará empíricamente que la acción reescaleada $\hat{I}$ del mapa simpléctico 4D es un proceso de difusión que satisface la ec. de F-P dada en (5.9). Como consecuencia, la acción original $I$ satisface una ecuación similar pero con un coeficiente de difusión proporcional a $\epsilon^{2}$. Experimentalmente, para este valor de $K$, esta relación resultó ser verdadera mientras $\epsilon \leq 10^{-2}$.

Se ha resuelto numéricamente la mencionada Ec. de F-P para un ensamble inicial con distribución Gaussiana de valor medio igual a 1.75 y desviación estándar igual a 0.1. Los valores de $\mathcal{D}(\hat{I})$ fueron aquellos de $\mathcal{D}_{n u}\left(I ; 10^{3}\right)$ graficados en la Fig. 5.10. Además se ha computado numéricamente la evolución según el mapa simpléctico $4 \mathrm{D}$ de un ensamble con $10^{5}$ partículas de prueba, con distribución similar en las acciones iniciales y con las condiciones iniciales en las otras variables elegidas así:

- $\theta_{0}=(1+\sqrt{5}) / 2 \approx 1.618$

- $J_{0}=3$

- $\psi_{0}$ elegidas uniformemente en $[0,2 \pi)$

Se utilizó $\epsilon=10^{-2}$ por lo que la relación entre el tiempo de F-P $(L)$ y el tiempo Hamiltoniano "real" $(n)$ es:

$$
L=\epsilon^{2} n=10^{-4} n .
$$

La Fig. 5.17 muestra tanto la solución de la ec. de F-P como los histogramas hechos con órbitas individuales, para tiempos $n$ iguales a 0 y 200 . Allí se puede ver que el comportamiento de la difusión de la acción en este mapa simpléctico sigue de cerca al comportamiento de la solución de la ec. de F-P.

Se ha superpuesto en negro el coeficiente de difusión utilizado para la integración de la ec. de F-P, de forma tal de mostrar esquemáticamente la razón por la cual el lado izquierdo de la distribución Gaussiana se difunde más fuertemente que el lado derecho de la misma. La Fig. 5.18 muestra las mismas cantidades para valores de $n$ iguales a 400, 1000 y 5000 .

Para finalizar con el análisis de la difusión en la capa gruesa, se ha computado la evolución temporal de la varianza del histograma, $\sigma^{2}$, para dos valores del parámetro perturbativo. En la Fig. 5.19-izq. se puede ver $\sigma^{2}(L)$, para $0 \leq L \leq 0.2$, para $\epsilon=10^{-3}$ y $\epsilon=10^{-4}$, en colores rojo y verde, respectivamente. Se ha utilizado la variable $L$ en el eje temporal a modo de tener un tiempo comparable entre ambos valores de $\epsilon$. Más específicamente, $L=0.3$ corresponde a $n=10^{6} \times 0.3=3 \times 10^{5}$ para $\epsilon=10^{-3}$, mientras que corresponde a $n=10^{8} \times 0.3=3 \times 10^{7}$ para $\epsilon=10^{-4}$. De la mencionada figura se observa que existe un acuerdo entre ambas evoluciones de la varianza.

En la Fig. 5.19-der. se muestra sólo la curva correspondiente a $\epsilon=10^{-3}$, para un rango temporal un orden de magnitud mayor: $0 \leq L \leq 2$. Se observa un comportamiento aproximadamente lineal de la varianza, con lo que se puede decir que bajo estas condiciones la difusión es aproximadamente normal.

\subsection{Capa estocástica delgada}

En el caso $K=3$ se tenía libertad para elegir las condiciones iniciales en cualquier parte del plano de fases del SM, siempre que se evitara las dos pequeñas islas de estabilidad que 


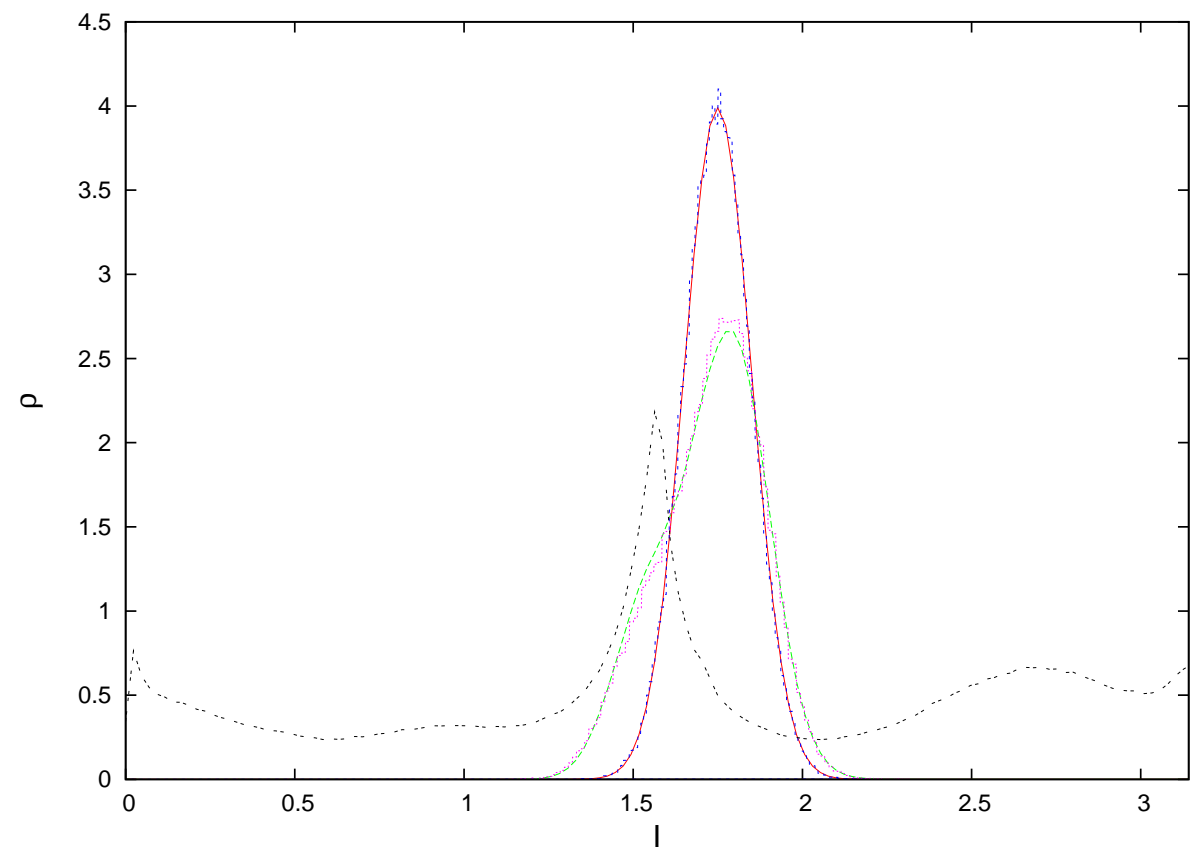

Figura 5.17: $\rho_{\epsilon}(I, n)$ para $n$ igual a 0 (rojo para la ec. de F-P, azul para histograma) y 200 (verde para la ec. de F-P, violeta para histograma), que se corresponde con $L$ igual a 0 y 0.02 , respectivamente. Para los histogramas se utilizó $K=3$ y $\epsilon=10^{-2}$. La línea negra superpuesta es el coeficiente de difusión utilizado al integrar la ec. de F-P.

se desarrollan para esa intensidad de $K$. Aquí, para un valor mucho más chico, $K=0.9$, se debe ser más específico y elegir las condiciones iniciales del ruido solamente dentro de la capa estocástica de una resonancia del mismo.

En esta oportunidad se eligió

$$
\mathcal{M} \equiv\left\{\left(J_{0}, \psi_{0}\right): \quad J_{0}=0.5, \quad 0.1 \leq \psi_{0} \leq 0.4\right\}
$$

En la Fig. 5.20 se muestra la ubicación inicial de dicho ensamble (en azul), junto con un snapshot del mismo, para un tiempo grande (en rojo).

\subsubsection{Funciones de correlación}

En la Fig. 5.21 se muestran las cuatro funciones de autocorrelación, definidas en Ec. (5.58), para $\tau \in[0,500]$ y para $t_{0}=10^{3}$, en colores rojo, azul, verde y negro, respectivamente. Análogamente a lo que sucedió para el caso de capa gruesa, se tiene que todas las autocorrelaciones menos $\phi_{2}$ oscilan alrededor de cero, y que $\phi_{3}$ junto con $\phi_{4}$ son las que presentan la menor amplitud de oscilación. Por otro lado, se tiene que $\phi_{1}$ y $\phi_{2}$ presentan oscilaciones del mismo orden de magnitud. Una característica distintitiva radica en que $\phi_{2}(\tau)$ posee un valor medio cercanano a 0.13 , que es bastante mayor que el valor medio correspondiente a la misma cantidad en el caso de capa gruesa. 


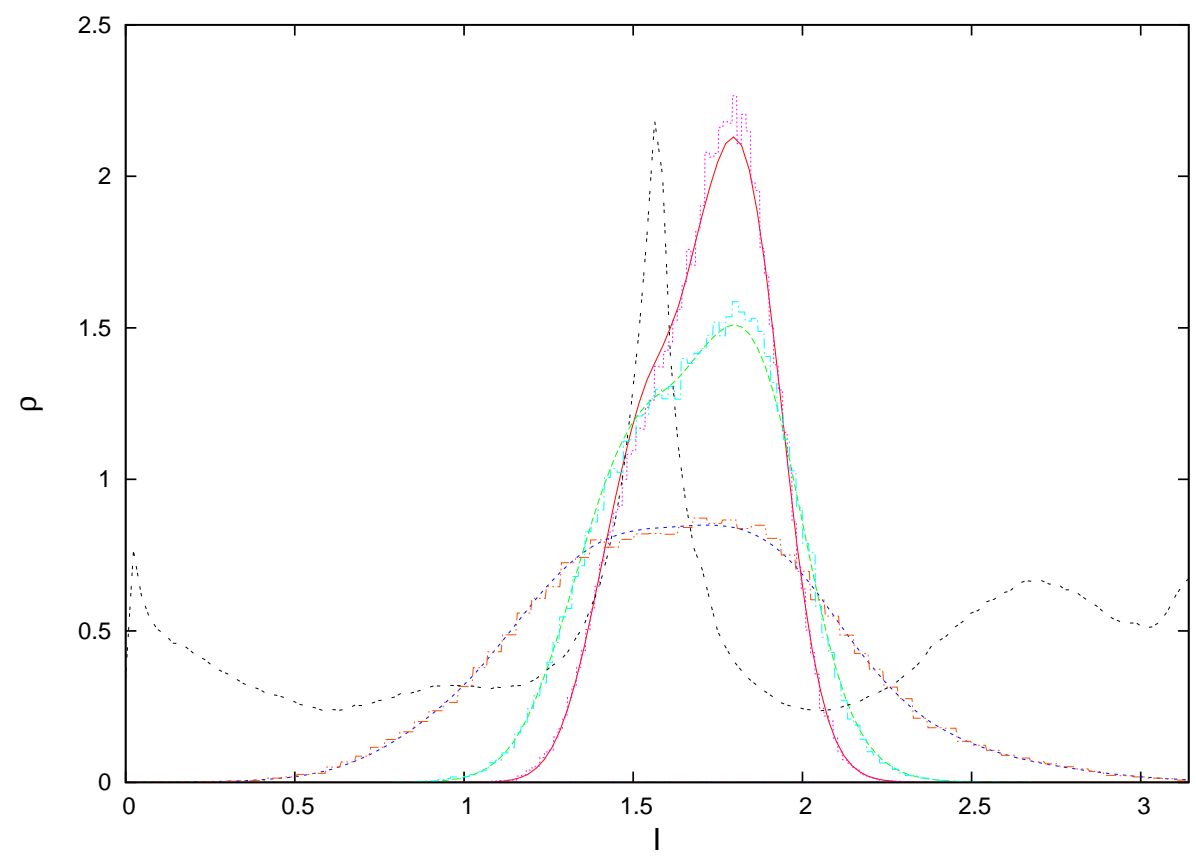

Figura 5.18: $\rho_{\epsilon}(I, n)$ para $n$ igual a 400,1000 y 5000, que corresponde con $L$ igual a 0.04, 0.1 y 0.5 , respectivamente. Para los histogramas se utilizó $K=3$ y $\epsilon=10^{-2}$. La línea negra superpuesta es el coeficiente de difusión utilizado al integrar la ec. de F-P.
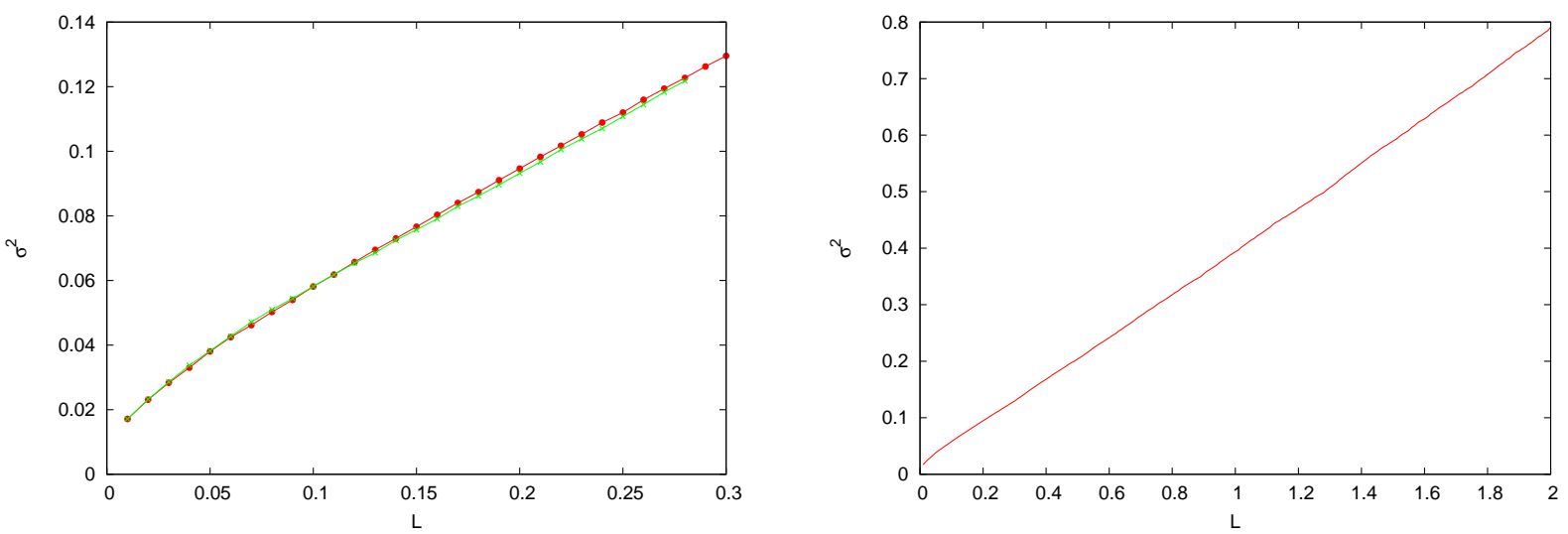

Figura 5.19: A la izquierda se despliega $\sigma^{2}(L)$, para $0 \leq L \leq 0.3$, para $\epsilon=10^{-3}$ y $\epsilon=10^{-4}$, en colores rojo y verde, respectivamente. A la derecha se despliega $\sigma^{2}(L)$, con $0 \leq L \leq 2$ y $\epsilon=10^{-3}$. Ambos gráficos han sido realizados utilizando la distribución correspondiente al histograma de $10^{5}$ partículas prueba, que fue definido anteriormente.

\subsubsection{Coeficientes de difusión}

Los resultados del cómputo de los coeficientes semi-numéricos y numéricos para $\epsilon=10^{-4}$ pueden ser apreciados en la Fig. 5.22, en escalas normal (izq.) y semi-logarítmica (der.), respectivamente. Podemos notar que existe un acuerdo, de menor calidad respecto al caso 


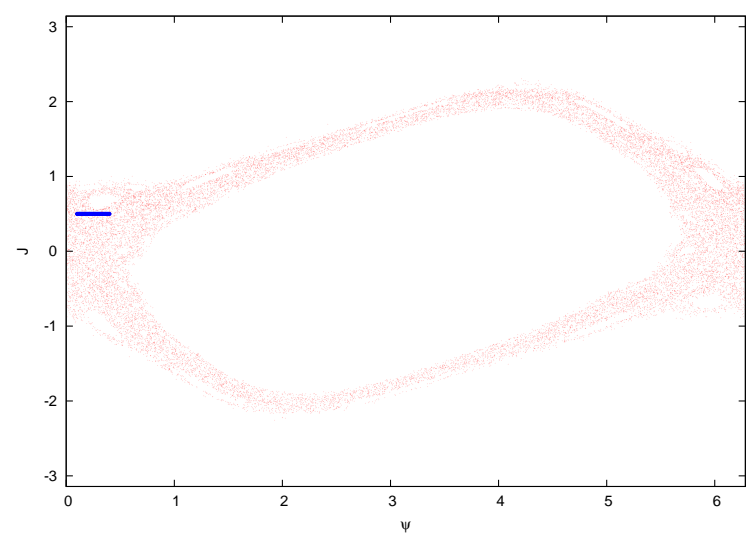

Figura 5.20: Condiciones iniciales del ruido (azul) y snapshot (rojo) corresondiente 700 unidades de tiempo, usando $K=0.90$ y $N_{r}=2 \times 10^{4}$.

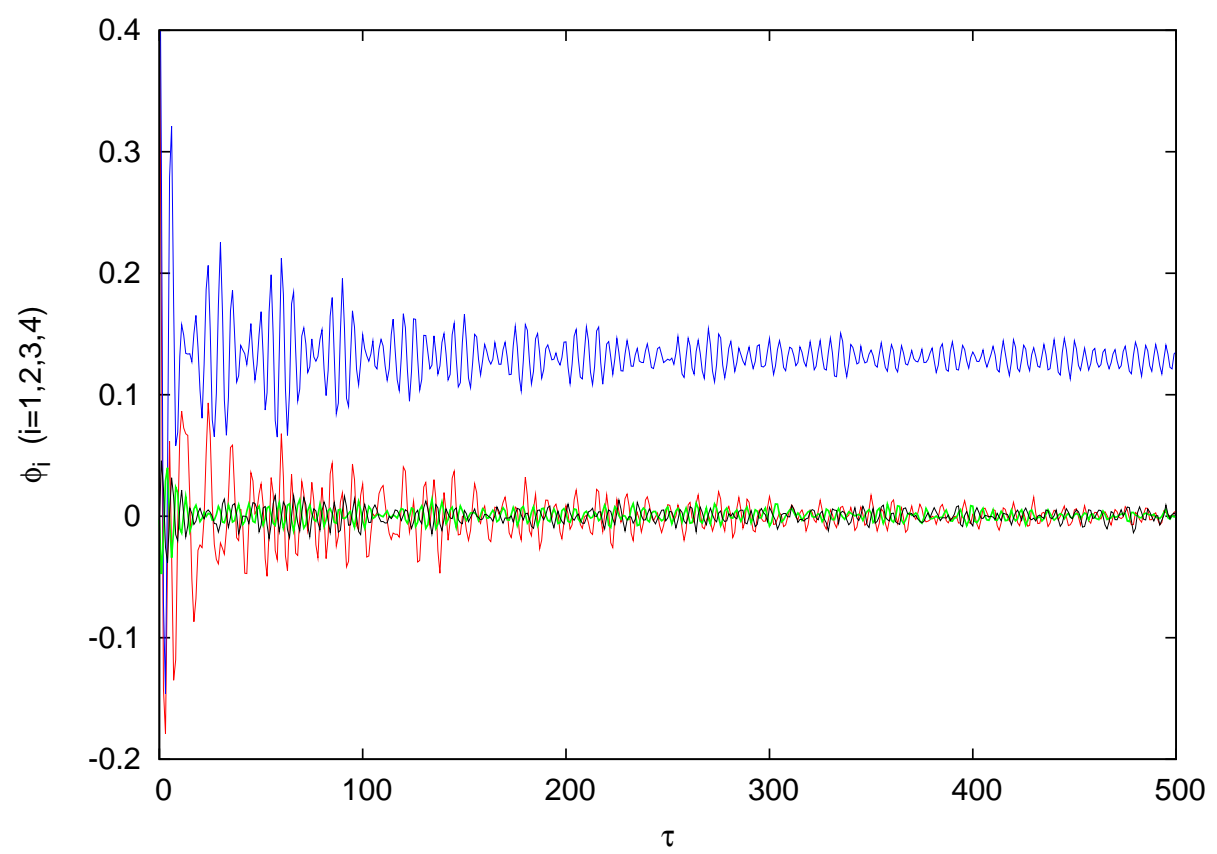

Figura 5.21: $\phi_{1}(\tau)$ (rojo), $\phi_{2}(\tau)$ (azul), $\phi_{3}(\tau)$ (verde) y $\phi_{4}(\tau)$ (negro), computadas utilizando un ensamble de $2 \times 10^{4}$ órbitas del SM $(K=0.9)$ con condiciones iniciales uniformemente distribuídas en $\mathcal{M}$.

$K=3$, entre la predicción (rojo) y el experimento (negro). Para el cálculo de $\mathcal{D}_{s n}^{\star}\left(I ; 10^{3}\right.$ ) se utilizaron las funciones numéricas $\phi_{i}$ que se obtuivieron en la subsección anterior.

\subsubsection{La densidad de probabilidad}

Nuevamente, se ha resuelto numéricamente la Ec. de F-P. Esta vez para un ensamble inicial con distribución Gaussiana con media igual a 0.75 y desviación estándar igual a 0.02. Los valores de $\mathcal{D}(\hat{I})$ fueron aquellos de $\mathcal{D}_{n u}\left(I ; 10^{3}\right)$ graficados en la Fig. 5.22. Además se han 

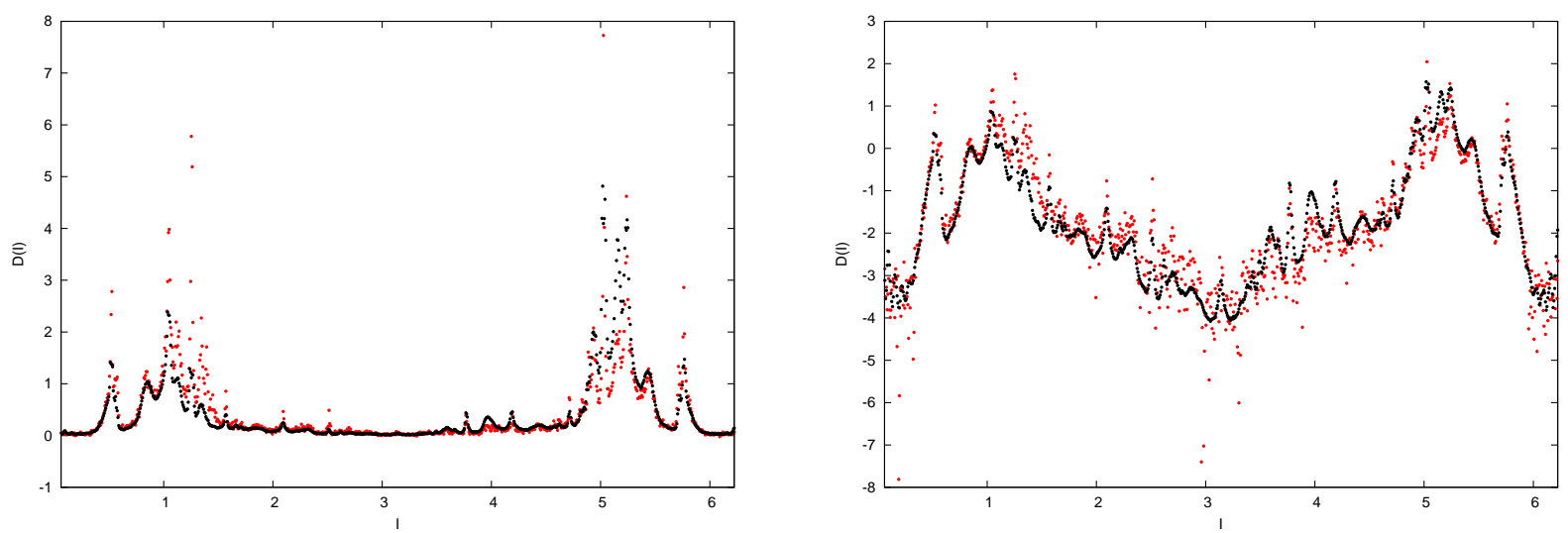

Figura 5.22: $\mathcal{D}_{s n}^{\star}\left(I ; 10^{3}\right)$ (rojo) y $\mathcal{D}_{n u}\left(I ; 10^{3}\right)$ (negro) para mapa simpléctico (5.50), usando $K=0.90, \epsilon=10^{-4}$ y $\theta_{0} \approx 0.214$, en escala natural (izquierda) y semilogarítmica (derecha).

computado numéricamente la evolución de un ensamble de $2 \times 10^{4}$ partículas de prueba con distribución similar en las acciones iniciales y con las condiciones iniciales de la otras variables dadas por:

- $\theta_{0}=(1+\sqrt{5}) / 2 \approx 1.618$

- $J_{0}=1 / 2$

- $\psi_{0}$ uniformemente elegidas en $[0.1,0.4]$.

Se ha utilizado $\epsilon=10^{-4}$ por lo que la relación entre el tiempo de F-P $(L)$ y $n$ es:

$$
L=\epsilon^{2} n=10^{-8} n .
$$

La Fig. 5.23 muestra la solución de la Ec. de F-P y los histogramas hechos con las órbitas individuales, para tiempos $n$ iguales a 0 y $10^{6}$. Se ha superpuesto en negro el coeficiente de difusión utilizado en la integración F-P. La Fig. 5.24 muestra las mismas cantidades para valores mayores de $n\left(3 \times 10^{6}\right.$ y $\left.8 \times 10^{6}\right)$

Tal como pasó para el valor de $K=3$, aquí se puede apreciar que la difusión percibida por la acción $I$ en este mapa simpléctico sigue de cerca al comportamiento de la solución de la ecuación cinética. Sin embargo, el histograma se presenta un poco retrasado respecto de la curva F-P. Tal vez este retraso se deba al hecho de que el sistema real (histograma) necesita un cierto tiempo para decorrelacionar los ángulos $(\psi)$, mientras van llenando la capa estocástica de la separatriz. Para poner a prueba esta hipótesis debería computarse la evolución del histograma utilizando un $\mathcal{M}$ donde las condiciones iniciales estén distribuídas lo más homogéneamente posible dentro de la capa estocástica.

Para finalizar con el análisis de la difusión en la capa fina, se ha computado la evolución temporal de la varianza de la distribución, $\sigma^{2}$, para dos valores del parámetro perturbativo. En la Fig. 5.25-izq. se puede ver $\sigma^{2}(L)$, para $0 \leq L \leq 0.3$, para $\epsilon=10^{-3}$ y $\epsilon=10^{-4}$, en colores rojo y verde, respectivamente. Se observa que ambas curvas comienzan a diverger desde $L \approx 0.05$. Esta diferencia puede deberse a que para este valor de $K$ el sistema se aleja demasiado de las hipótesis del teorema estocástico. Comparando entre sí las Figs. 5.15 


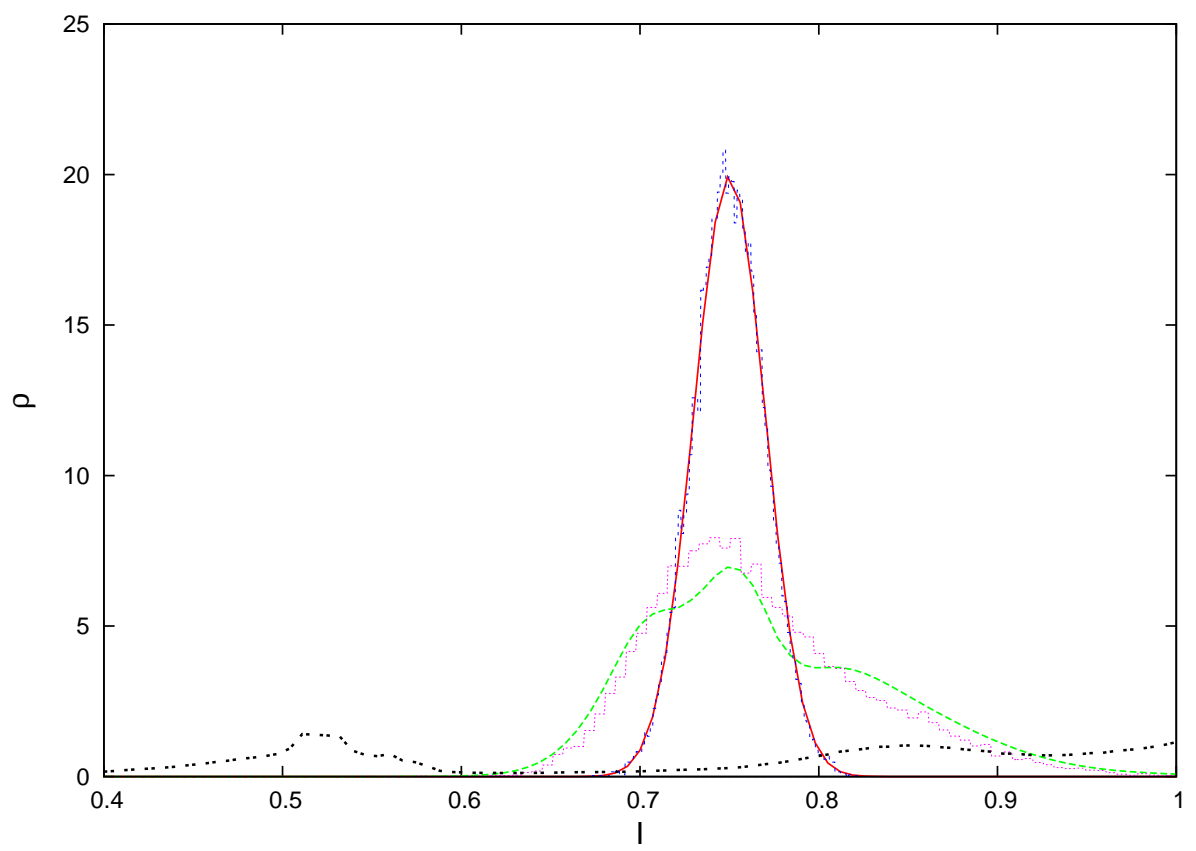

Figura 5.23: $\rho_{\epsilon}(I, n)$ para $n$ igual a 0 (rojo para la ec. de F-P, azul para histograma) y $10^{6}$ (verde para la ec. de F-P, violeta para histograma), que se corresponde con $L$ igual a 0 y 0.01 , respectivamente. Para los histogramas se utilizó $K=0.9$ y $\epsilon=10^{-4}$. La línea negra superpuesta es el coeficiente de difusión utilizado al integrar la ec. de F-P.

y 5.21 se concluye que para el caso de capa fina el no decaimiento a cero de las funciones de autocorrelación es más evidente que en el caso de capa gruesa. En particular, para $K=0.9$, $\phi_{2}(\tau)$ está centrada en un valor de casi un orden de magnitud mayor al correspondiente para $K=3$, como fue antes mencionado.

Tanto para $K=3$ como para $K=0.9$, la persistencia de correlaciones para todo tiempo, se debe a la existencia de islas de estabilidad ya que las partículas de la capa estocástica, al acercarse suficientemente cerca a las mismas, presentan un comportamiento con características regulares, y la dinámica se "ordena" localmente allí. En el caso de capa fina, existe una mayor componente de islas de estabilidad en el espacio de fases, como puede verse en la Fig. 5.20.

En la Fig. 5.25-der. se muestra el histograma de la densidad de probabilidad, para $L=0.3$, para los dos valores de $\epsilon$ recientemente considerados. El histograma rojo es $\rho_{\epsilon}\left(I, 3 \times 10^{5}\right)$ y corresponde a $\epsilon=10^{-3}$, mientras que el histograma verde es $\rho_{\epsilon}\left(I, 3 \times 10^{7}\right)$ y corresponde a $\epsilon=10^{-4}$. La curva negra es la correspondiente solución de la ecuación de F-P: $\rho(I, 0.3)$. De esta figura se concluye que, si bien la diferencia de las varianzas de ambos histogramas, es perceptible, la ec. de F-P logra modelar el comportamiento general de la distribución macroscópica del ensamble para tiempos del orden del $L$ considerado. De forma similar, en la Fig. 5.26-izq. se muestra el histograma $\rho_{\epsilon}\left(I, 2 \times 10^{6}\right)$, para a $\epsilon=10^{-3}$, junto con la solución de la ecuación de F-P, $\rho(I, 2)$, obteniéndose un buen acuerdo entre ambas curvas.

En la Fig. 5.26-der. se muestra $\sigma^{2}(L)$ correspondiente a $\epsilon=10^{-3}$, para el rango temporal $0 \leq L \leq 2$. Se observa que la evolución de la varianza no es lineal por lo que la difusión resulta ser anómala. La varianza tampoco obedece una ley de potencias, pero se puede decir que el comportamiento es subdifusivo en el intervalo temporal considerado. 


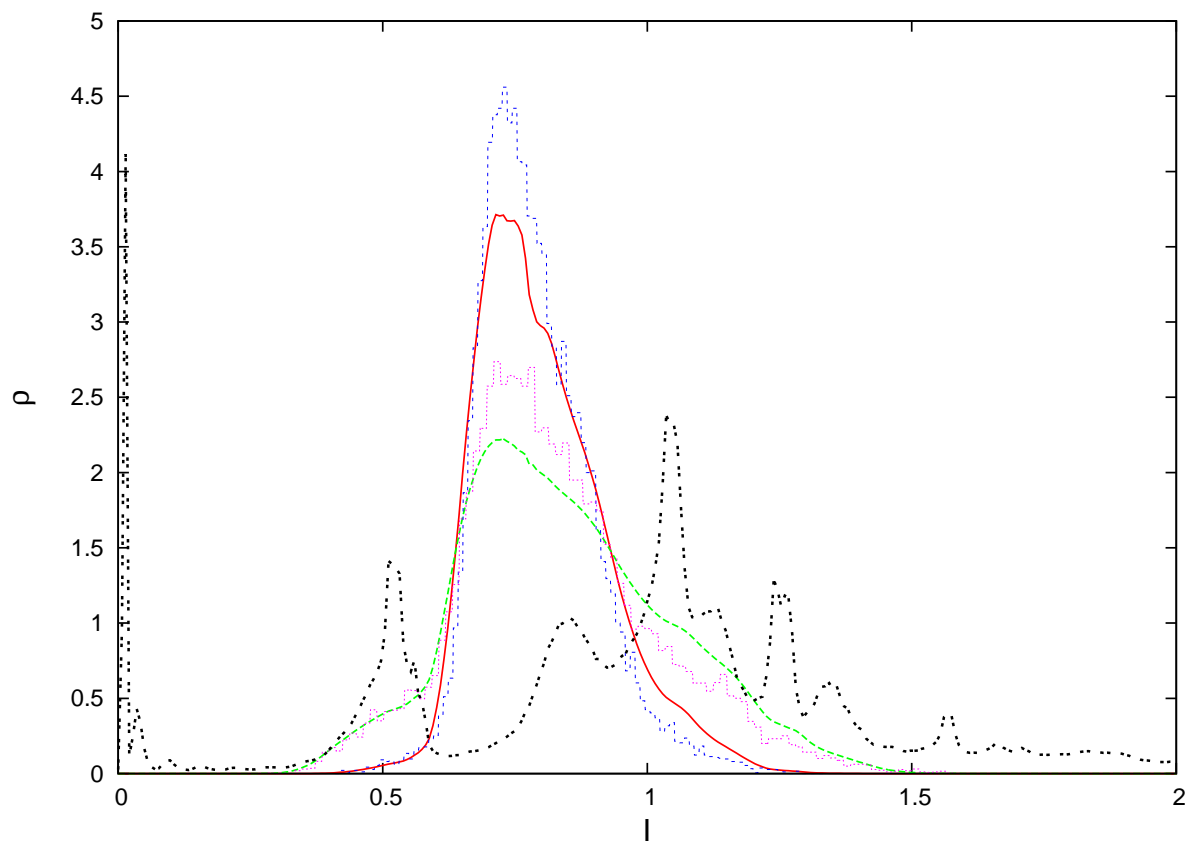

Figura 5.24: $\rho_{\epsilon}(I, n)$ para $n$ igual a $3 \times 10^{6}$ (rojo para F-P, azul para histograma) y $8 \times$ $10^{6}$ (verde para F-P, violeta para histograma), que se corresponde con $L$ igual a 0.03 y 0.08 , respectivamente. Para los histogramas se utilizó $K=0.9$ y $\epsilon=10^{-4}$. La línea negra superpuesta es el coeficiente de difusión utilizado al integrar la Ec. F-P.
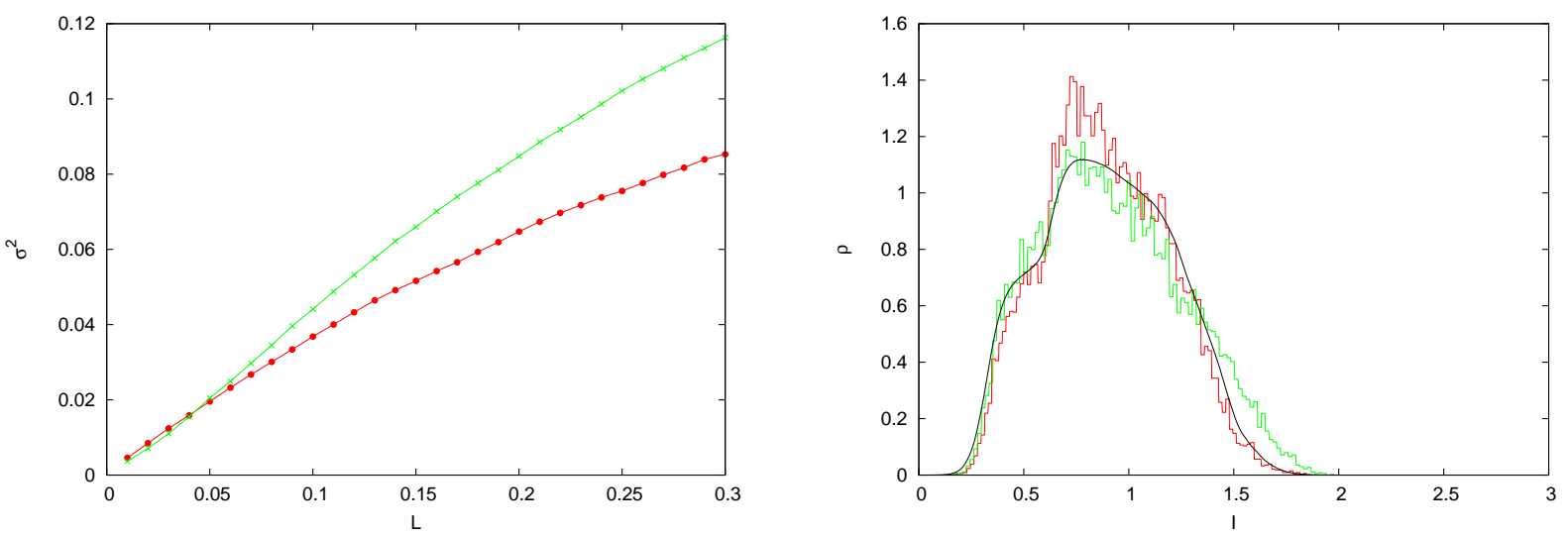

Figura 5.25: A la izquierda se despliega $\sigma^{2}(L)$, con $0 \leq L \leq 0.3$, para $\epsilon=10^{-3}$ y $\epsilon=10^{-4}$, en colores rojo y verde, respectivamente. A la derecha se despliega, en rojo, el histograma $\rho_{\epsilon}(I, 3 \times$ $\left.10^{5}\right)$, correspondiente a $\epsilon=10^{-3}$, y en verde, el histograma $\rho_{\epsilon}\left(I, 3 \times 10^{7}\right)$, correspondiente a $\epsilon=10^{-4}$. La curva negra es la corresponde a la solución de la ecuación de F-P: $\rho(I, 0.3)$.

\subsection{Conclusión}

En este capítulo se ha estudiado la característica difusiva de una quasi-acción $(I)$ de un mapa simpléctico 4D a priori inestable. 

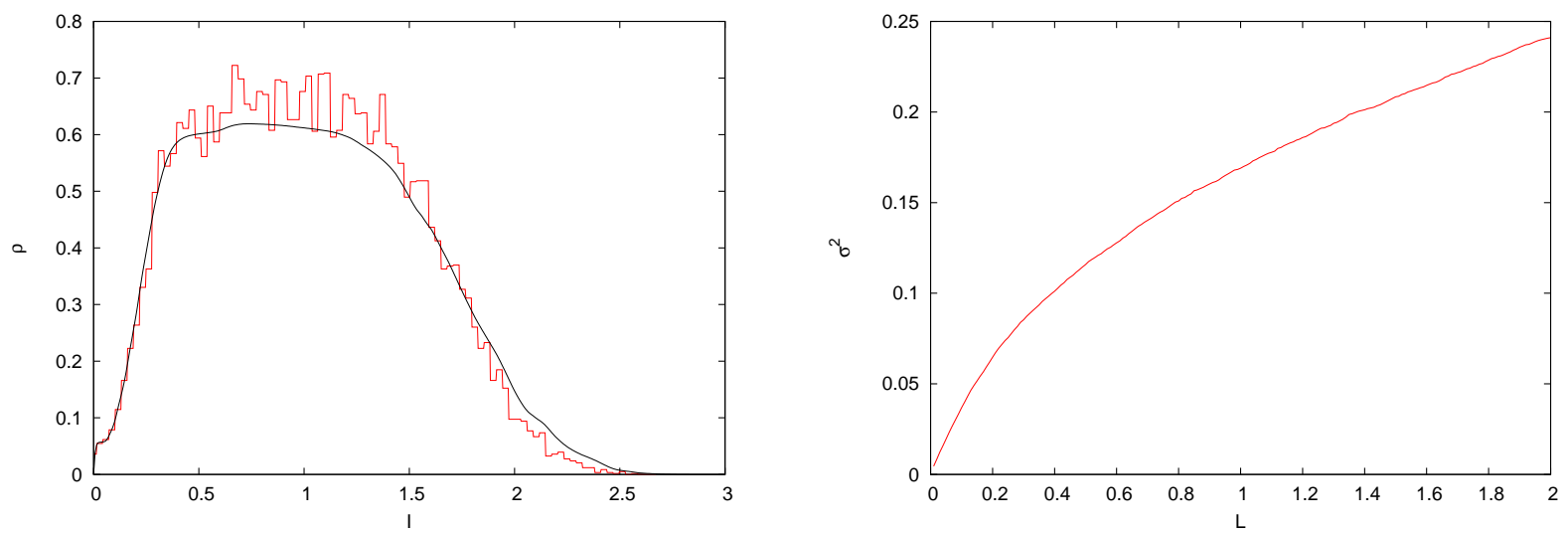

Figura 5.26: A la izquierda se despliega, en rojo, el histograma $\rho_{\epsilon}\left(I, 2 \times 10^{6}\right)$, correspondiente a $\epsilon=10^{-3}$, y en negro, la solución de la ecuación de F-P: $\rho(I, 2)$. A la derecha se despliega $\sigma^{2}(L)$, con $0 \leq L \leq 2$ y $\epsilon=10^{-3}$.

En todo momento se trabajó con partículas de prueba caóticas, ya que siempre $\mathcal{M}$ fue elegido de forma tal que las condiciones iniciales en el plano $[J, \psi]$ pertenecieran a la capa estocástica del SM.

Se utilizó como herramienta, para predecir el coeficiente de difusión, un método seminumérico basado en un teorema para sistemas Hamiltonianos perturbados estocásticamente. Dicho método permitió predecir aproximadamente el coeficiente de difusión en dos valores de $K$, que corresponden con las situaciones de capa gruesa y de capa delgada, para un rango amplio de valores de $\epsilon$. Las situaciones de capa gruesa y delgada, poseen una geometría similar al régimen de Chirikov y de Nekhoroshev en sistemas Hamiltonianos casi-integrables, respectivamente.

Aunque para el mapa simpléctico no se utilizó una expresión analítica, el coeficiente de difusión semi-numérico es una predicción porque utiliza como datos de entrada sólamente al movimiento no perturbado de la parte integrable (rotador libre) y a la función de autocorrelación numérica de la perturbación (SM).

A su vez, se probó experimentalmente, que la evolución macroscópica de una dada distribución $\rho_{\epsilon}(I, n)$ satisface aproximadamente la ecuación de F-P. Se observó que, en el caso de capa delgada, la evolución de la varianza es levemente sensible al valor de $\epsilon$ utilizado. Esto concuerda con el hecho de que para este caso, el ruido del SM se aleja demasiado de las condiciones del teorema estocástico, principalmente en lo relativo a la necesidad de que la función de correlación decaiga a cero con el tiempo; situación que no sucede debido a la densidad y al tamaño de las islas de estabilidad en la capa estocástica.

Finalmente, en el caso de capa gruesa la difusión se comportó aproximadamente normal, mientras que en el otro se obtuvo un comportamiento subdifusivo. Esto último concuerda con el comportamiento obtenido para sistemas Hamiltonianos 3DoF, en donde la difusión que tuvo lugar a lo largo de la resonancia guía, para ensambles pertenecientes a la capa estocástica, en régimen de Nekhoroshev, también tenía características subdifusivas. 


\section{Conclusiones y perspectivas}

En esta tesis se ha trabajado con el objetivo de responder aquellas preguntas planteadas en el Prólogo referidas a la descripción y modelado de la evolución de quasi-ensambles de partículas de prueba en sistemas Hamiltonianos casi-integrables.

Lejos de intentar lograr afirmaciones universalmente válidas, se focalizó en dos sistemas Hamiltonianos particulares. Sin embargo, debido a que los mismos modelan con suficiente generalidad la geometría resonante de sistemas Hamiltonianos con espacio de fases dividido, resulta plausible que la información de los procesos difusivos aquí obtenida sea parcialmente generalizable.

Una de las particularidades que se presentaron durante el desarrollo de esta tesis, fue el carácter abstracto de los objetos de estudio, como lo son un sistema de ecuaciones diferenciales ordinarias Hamiltonianas o un mapa simpléctico. La necesidad de medir cantidades que dependen de trayectorias que no se pueden conocer, y que justamente por esta razón son interesantes, sugirió la necesidad de adoptar como nuevo objeto de estudio, a las soluciones numéricas (del sistema Hamiltoniano) asociadas a un dado código integrador (o iterador), a una dada aritmética de CPU y a un tipo de hardware. Por lo tanto, utilizando la habitual suposición de que las cantidades medidas en estas soluciones numéricas representan fielmente a la realidad del sistema Hamiltoniano original, es posible clasificar a la mayoría de las tareas desarrolladas a lo largo de esta tesis, en dos grupos: tareas de predicción y tareas de medición.

En cada capítulo se brindó una discusión de los resultados allí obtenidos. Por ello, a continuación se resumirán las principales conclusiones de los mismos.

En el capítulo 1 se introdujeron algunos conceptos teóricos. Fundamentalmente, allí se estableció la situación geométrica general en la cual se trabajó posteriormente. Se clasificó, en líneas generales, a los sistemas Hamiltonianos casi-integrables, en dos regímenes. Aquellos con parámetro perturbativo $(\epsilon)$ suficientemente grande como para que la mayoría de las condiciones iniciales pertenezcan a alguna capa estocástica, que en algunos casos deviene en el llamado mar caótico, se clasificaron como pertencientes al régimen de Chirikov. Por otro lado, aquellos con $\epsilon$ en el complemento del conjunto anterior, se clasificaron como pertenecientes al régimen de Nekhoroshev. Posteriormente, se habló acerca de la posibilidad de tener difusión en ciertas regiones del espacio de acciones, con características propias del régimen del sistema. En particular, se desarrollaron las limitaciones a la difusión y al transporte que existen para el régimen de Nekhoroshev, caracterizando la geometría del subconjunto del espacio de fases habilitado a tal fin. Tal subconjunto es la Red de Arnold. Además, se presentan algunas de 
las teorías existentes para la difusión de Arnold.

En el capítulo 2 se introducen algunos indicadores dinámicos variacionales. Los mismos permitieron, entre otras cosas, generar la proyección de la estructura resonante sobre la superficie de energía no perturbada $\left(\mathcal{I}_{0}\right)$. Además, se presenta el descubrimiento de una relación analítica exacta entre dos de estos indicadores, el FLI y el MEGNO. Esta relación analítica permitió predecir el comportamiento de la primera de ellas, en términos de la segunda, lo cual fue corroborado al medir estas cantidades en simulaciones numéricas en el mapa estándar $(\mathrm{SM})$

El capítulo 3 tuvo tres objetivos principales. Uno de ellos fue desarrollar un conocimiento analítico suficientemente amplio sobre el sistema Hamiltoniano con tres grados de libertad (3DoF) con el que se deseaba trabajar. Se trató de tres osciladores cuárticos acoplados por una perturbación no integrable, que deja como única integral global del sistema a la energía total. La parte integrable de este flujo Hamiltoniano es no lineal y posee una superficie de energía convexa en el espacio de acciones.

Otro de ellos consistió en poner a prueba, por primera vez para este sistema, el criterio de overlap de Chirikov, como método predictor del valor del $\epsilon$ crítico $\left(\epsilon_{c}\right)$ que separa entre sí a los dos regímenes globales de difusión. La metodología aplicada en esta parte fue en su mayoría analítica, recurriéndose al cómputo numérico sólo para evaluar las fórmulas teóricas. Dichas estimaciones fueron comparadas con una medición de la fracción de órbitas caóticas que había sido previamente realizada en la literatura. La Fig. 3.9 muestra la equivalencia funcional entre predicción y medición.

El tercer objetivo consistió en poder predecir la estructura resonante mediante la utilización de la aproximación del péndulo simple, trabajando con formas normales resonantes a primer y segundo orden en $\epsilon$. La Fig. 3.8 hace la comparación, para un valor de fijo de $\epsilon$, entre los cálculos semi-analíticos y la estructura resonante obtenida, también previamente en la literatura, utilizando el MEGNO.

El capítulo 4 es netamente experimental y, debido a la necesidad de realizar mediciones precisas, se dedicó un tiempo significativo a la búsqueda y prueba de integradores de sistemas de ecuaciones diferenciales ordinarias. Los conocimientos teóricos fueron utilizados para elegir la resonancia guía, para identificar las capas estocásticas, para seleccionar ensambles de partículas de prueba dentro de las mismas y para saber en qué dirección medir la difusión. Además, se utilizaron para saber qué formas funcionales de las evoluciones temporales de algunas cantidades estadísticas es plausible ajustar.

Se encontró que existen ciertos rangos temporales en los cuales la varianza no se comporta, en forma efectiva, linealmente con el tiempo. Este resultado suma en la dirección de trabajos previos de la literatura que intentan poner a prueba la idea de que los sistemas deterministas pueden presentar aspectos anómalos en su difusión. En el experimento $\mathscr{A}$ se midieron exponentes de Hurst asociados a la varianza en la dirección paralela a la resonancia $\left(\sigma_{3}^{2}\right)$, obteniéndose en general, que dicha cantidad crece con $\epsilon$.

Otro resultado de este capítulo fue la identificación de rangos temporales en los cuales $\sigma_{3}^{2}$ se comportaba aproximadamente como una función lineal del tiempo. Es decir, se identificaron comportamientos de difusión normal, para posteriormente medir los coeficientes de difusión $\mathcal{D}_{\sigma}$. Esta tarea se realizó para distintos valores de $\epsilon$ en los experimentos $\mathscr{A}$ y $\mathscr{B}$, fijando la acción resonante en ambos casos, aunque en distintos valores. Si bien no se pudo hacer un ajuste numérico de la dependencia del coeficiente con $\epsilon$, sí se pudo verificar que dicho coeficiente decrece al disminuir $\epsilon$. En el experimento $\mathscr{C}$ se fijó el valor $\epsilon$ en uno que corresponde al régimen de Nekhoroshev, midiéndose dicho coeficiente para diez ensambles correspondientes 
a diferentes acciones resonantes, ubicadas a lo largo de la resonancia. Otro resultado obtenido fue el cambio cualitativo en el comportamiento de $\sigma_{3}^{2}(t)$ según la distancia del ensamble inicial respecto a cruces con otras resonancias.

Finalmente, en el capítulo 5, mediante la aplicación heurística de un método basado en un teorema para sistemas Hamiltonianos perturbados estocásticamente (no completamente deterministas), se realizaron dos tipos de tareas en un mapa simpléctico 4D a priori inestable. Dicho mapa consiste en un rotador libre acoplado con un SM. Se trabajó con dos valores del parámetro $K$ del SM, que corresponden con los regímenes de capa gruesa y capa fina. La geometría del espacio de fases para estos dos valores de $K$, presenta una similaridad con la geometría presente en flujos Hamiltonianos casi-integrables, respectivamente, en los regímenes de Chirikov y Nekhoroshev. Este tipo de mapa ha sido estudiado previamente en la literatura, al poner a prueba el modelo de pumping estocástico, y existen predicciones del coeficiente de difusión en modelos similares.

Se estimó el coeficiente de difusión, asociado a la acción $(I)$ del rotador libre, con técnica semi-numérica, y posteriormente fue comparado con mediciones numéricas del mismo. Además, se predijo mediante la solución de una ecuación de Fokker-Planck, la evolución de la función densidad de probabilidad de $I$. Esto fue corroborado mediante el cómputo de histogramas de un ensamble de partículas con distribución inicial similar a la ingresada en la Ec. de F-P.

En resumen, se verificó experimentalmente que para el sistema determinista estudiado, se cumplen aproximadamente las predicciones de un teorema cuya validez teórica está limitada a sistemas casi-integrables perturbados estocásticamente.

Entre las perspectivas de trabajo a futuro relacionadas con el sistema 3DoF, se encuentran:

- Realizar cómputos de $\mathcal{D}_{\sigma}$ en un conjunto de valores de $\epsilon$ más amplio, alcanzando valores suficientemente chicos como para poder ajustar la dependencia.

- Analizar la dependencia de dicho coeficiente y del exponente de Hurst respecto a la ubicación de la acción resonante elegida.

- Comparar estas mediciones con

1. las realizadas en las variables correspondientes al órden óptimo de normalización.

2. la predicción de la teoría de Chirikov.

Por otro lado, se pretende poner a prueba el método estocástico en otros mapas simplécticos más generales y en flujos Hamiltonianos casi-integrables. 


\section{Bibliografía}

Arnold, L.: 1973, Stochastic Differential Equations: theory and applications. John Wiley \& Sons.

Arnold, V. I.: 1964, 'Instability of dynamical systems with several degrees of freedom'. Sov. Math.-Dokl. 5, 581-585.

Arnold, V. I.: 1989, MATHEMATICAL METHODS OF CLASSICAL MECHANICS. New York: Springer-Verlag, second edition.

Barrio, R., W. Borczyk y S. Breiter: 2009, 'Spurious structures in chaos indicators maps'. Chaos Solitons \& Fractals 40, 1697-1714.

Bartle, R. G.: 1995, The elements of integration and Lebesgue measure. New York: John Wiley \& Sons.

Bauer, M., R. Valiullin, G. Radons y J. Kärger: 2011, 'How to compare diffusion processes assessed by single-particle tracking and pulsed field gradient nuclear magnetic resonance'. J. Chem. Phys. 135(14), 144118.

Bazzani, A. y L. Beccaceci: 1998, 'Diffusion in Hamiltonian systems driven by harmonic noise'. J. Phys. A - Math. Gen. 31, 5843-5854.

Bazzani, A., L. Beccaceci, L. Bigliardi y G. Turchetti: 1997, 'Fokker-Planck solutions for action diffusion in a noisy symplectic map'. In: American Institute of Physics Conference Series, Vol. 395. pp. 109-118.

Bazzani, A. y H. Mais: 1998, 'Effect of colores noise on the betatronic motion: a possible mechanism for slow extraction.'. In: A. Bazzani, J. Ellison, H. Mais y G. Turchetti (eds.): Nonlinear and stochastic beam dynamics in accelerators - A chalenge to theoretical and computational physics.

Beccaceci, L.: 1996, 'Diffusione in modelli Hamiltoniani in presenza di rumore correlato'. Graduation thesis in physics, University of Bologna. 
Benettin, G. y G. Gallavotti: 1986, 'Stability of motions near resonances in quasi-integrable Hamiltonian systems'. J. Stat. Phys. 44, 293-338.

Birkhoff, G. D.: 1927, Dynamical Systems. New York: American Mathematical Society.

Bountis, T., H. Isliker, M. Kollmann y M. Vrahatis: 1998, 'Stability and diffusion in 4-D mapping models of accelerator dynamics'. In: A. Bazzani, J. Ellison, H. Mais y G. Turchetti (eds.): Nonlinear and stochastic beam dynamics in accelerators - A chalenge to theoretical and computational physics.

Bountis, T. y M. Kollmann: 1994, 'Diffusion rates in a 4-dimensional mapping model of accelerator dynamics'. Physica D 71, 122-131.

Breiter, S., M. Fouchard y R. Ratajczak: 2008, 'Stationary orbits of comets perturbed by Galactic tides'. MNRAS 383, 200-208.

Cachucho, F., P. M. Cincotta y S. Ferraz-Mello: 2010, 'Chirikov diffusion in the asteroidal three-body resonance (5, -2,-2)'. Celest. Mech. Dyn. Astron. 108, 35-58.

Cary, J. R., J. D. Meiss y A. Bhattacharjee: 1981, 'Statistical characterization of periodic, area-preserving mappings'. Phys. Rev. A 23, 2744-2746.

Chechkin, A. V., R. Metzler, J. Klafter y V. Y. Gonchar: 2008, Introduction to the Theory of Lévy Flights, pp. 129-162. Wiley-VCH Verlag GmbH \& Co. KGaA.

Chierchia, L.: 1995, 'Arnold Instability For Nearly-Integrable Analytic Hamiltonian Systems'.

Chierchia, L. y G. Gallavotti: 1994, 'Drift and diffusion in phase space'. Annales de l'I.H.P. Physique théorique 60(1), 1-144.

Chirikov, B. V.: 1979, 'A universal instability of many-dimensional oscillator systems'. Phys. Rep. 52, 263-379.

Chirikov, B. V., J. Ford y F. Vivaldi: 1980, 'Some numerical studies or arnold diffusion in a simple model'. In: American Institute of Physics Conference Series, Vol. 57. pp. 323-340.

Christodoulidi, H. y T. Bountis: 2006, 'Low-dimensional quasiperiodic motion in Hamiltonian systems'. ROMAI J. 2, 37-44.

Cincotta, P. M.: 2002, 'Arnold diffusion: an overview through dynamical astronomy'. New Astron. Rev. 46, 13-39.

Cincotta, P. M., C. M. Giordano y C. Simó: 2003, 'Phase space structure of multi-dimensional systems by means of the mean exponential growth factor of nearby orbits'. Physica D 182, $151-178$.

Cincotta, P. M. y C. Simó: 2000, 'Simple tools to study global dynamics in non-axisymmetric galactic potentials - I'. A\&AS 147, 205-228.

Cogburn, R. y J. A. Ellison: 1992, 'A stochastic theory of adiabatic invariance'. Commun. Math. Phys. 149, 97-126.

Cordeiro, R. R.: 2006, 'Anomalous Diffusion in the Asteroid Belt'. AJ 132, 2114-2126. 
Cordeiro, R. R. y L. A. Mendes de Souza: 2005, 'Anomalous diffusion in the first-order Jovian resonance'. A\& $A$ 439, 375-385.

Darriba, L.: 2008, 'Tratamiento numérico de ecuaciones diferenciales con aplicaciones a sistemas dinámicos'. Tesis de grado en astronomía, FCAGLP, UNLP, Argentina.

Denisov, S., J. Klafter y M. Urbakh: 2002, 'Ballistic flights and random diffusion as building blocks for Hamiltonian kinetics'. Phys. Rev. E 66(4), 046217-+.

Easton, R. W., J. D. Meiss y G. Roberts: 2001, 'Drift by coupling to an anti-integrable limit'. Physica D Nonlinear Phenomena 156, 201-218.

Eckmann, J. P. y D. Ruelle: 1985, 'Ergodic theory of chaos and strange attractors'. Rev. Mod. Phys. 57, 617-656.

Efthymiopoulos, C.: 2008, 'On the connection between the Nekhoroshev theorem and Arnold diffusion'. Celest. Mech. Dyn. Astron. 102, 49-68.

Efthymiopoulos, C.: 2010, 'Special features of galactic dynamics: Disc dynamics'. European Physical Journal Special Topics 186, 91-122.

Efthymiopoulos, C., N. Voglis y C. Kalapotharakos: 2007, 'Special Features of Galactic Dynamics'. In: Lecture Notes in Physics, Berlin Springer Verlag, Vol. 729 of Lecture Notes in Physics, Berlin Springer Verlag. pp. 297-389.

Einstein, A.: 1956, On the movement of small particles suspended in a stationary liquid demanded by the molecular kinetic theory of heat. Traducción de Annalen der Physik (Leipzig), Vol. 17 (1905), pp. 549-560, por D. Cowper., pp. 1-18. New York: Dover Publications.

Faranda, D., M. F. Mestre y G. Turchetti: 2012, 'Analysis or round off errors in symplectic maps with reversibility test'. In: G. Nicolis, M. Robnik, V. Rothos y H. Skokos (ed.): Special Issue of the International Journal of Bifurcation and Chaos, en prensa.

Ferraz-Mello, S.: 2007, CANONICAL PERTURBATION THEORIES - Degenerate Systems and Resonance. New York: Springer.

Flach, S. y A. Ponno: 2008, 'The Fermi Pasta Ulam problem: Periodic orbits, normal forms and resonance overlap criteria'. Physica D 237, 908-917.

Fox, R. F., I. R. Gatland, R. Roy y G. Vemuri: 1988, 'Fast, accurate algorithm for numerical simulation of exponentially correlated colored noise'. Phys. Rev. A 38, 5938-5940.

Froeschlé, C., R. Gonczi y E. Lega: 1997a, 'The fast Lyapunov indicator: a simple tool to detect weak chaos. Application to the structure of the main asteroidal belt'. Planet. Space Sci. 45, 881-886.

Froeschlé, C., M. Guzzo y E. Lega: 2000, 'Graphical Evolution of the Arnold Web: From Order to Chaos'. Science 289, 2108-2110.

Froeschlé, C., M. Guzzo y E. Lega: 2005, 'Local And Global Diffusion Along Resonant Lines in Discrete Quasi-integrable Dynamical Systems'. Celest. Mech. Dyn. Astron. 92, 243-255. 
Froeschlé, C. y E. Lega: 2000, 'On the Structure of Symplectic Mappings. The Fast Lyapunov Indicator: a Very Sensitive Tool'. Celest. Mech. Dyn. Astron. 78, 167-195.

Froeschlé, C., E. Lega y R. Gonczi: 1997b, 'Fast Lyapunov Indicators. Application to Asteroidal Motion'. Celest. Mech. Dyn. Astron. 67, 41-62.

Gardiner, P. R.: 2004, Handbook of Stochastic Methods. Berlin Heidelberg: Springer-Verlag.

Gillespie, D. T.: 1996a, 'Exact numerical simulation of the Ornstein-Uhlenbeck process and its integral'. Phys. Rev. E 54, 2084-2091.

Gillespie, D. T.: 1996b, 'The mathematics of Brownian motion and Johnson noise'. Am. J. Phys. 64, 225-240.

Giordano, C. M. y P. M. Cincotta: 2004, 'Chaotic diffusion of orbits in systems with divided phase space'. A\&3A 423, 745-753.

Giorgilli, A.: 1990, 'New Insights on the Stability problem from recent results in Classical Perturbation Theory'. In: D. Benest y C. Froeschlé (eds.): Modern Methods in Celestial Mechanics, Comptes Rendus de la 13ieme Ecole Printemps d'Astrophysique de Goutelas (France), 24-29 Avril, 1989. Gif-sur-Yvette: Editions Frontieres. p. 249.

Gradshtyn, I. S. y I. M. Ryzhik: 1980, Table of Integrals, Series and Products. New York: Academic Press.

Greene, J. M.: 1979, 'A method for determining a stochastic transition'. J. Math. Phys. 20, $1183-1201$.

Guzzo, M., E. Lega y C. Froeschlé: 2002, 'On the numerical detection of the effective stability of chaotic motions in quasi-integrable systems'. Physica D 163, 1-25.

Guzzo, M., E. Lega y C. Froeschlé: 2005, 'First numerical evidence of global Arnold diffusion in quasi-integrable systems'. Disc. Cont. Dyn. Syst.-B 5, 687-698.

Guzzo, M., E. Lega y C. Froeschlé: 2009, 'A Numerical Study of Arnold Diffusion in a Priori Unstable Systems'. Communications in Mathematical Physics 290, 557-576.

Halmos, P. R.: 1956, Lectures on ergodic theory. New York: Chelsea Publishing Company.

Higham, D. J.: 2001, 'An Algorithmic Introduction to Numerical Simulation of Stochastic Differential Equations'. SIAM Review 43, 525-546.

Hinse, T. C., A. A. Christou, J. L. A. Alvarellos y K. Goździewski: 2010, 'Application of the MEGNO technique to the dynamics of Jovian irregular satellites'. MNRAS 404, 837-857.

Jorba, À. y Z. Maorong: 2005, 'A software package for the numerical integration of ODE by means of high-order Taylor methods'. Experiment. Math. 14, 99-117.

Khasminskii, R. Z.: 1966, 'A limit theorem for the solutions of differential dquations with random right-hand sides'. Theory Probab. Appl. 11, 390-406.

Klafter, J., M. F. Shlesinger y G. Zumofen: 1996, 'Beyond Brownian motion'. Phys. Today 49, 33-39. 
Klafter, J. y G. Zumofen: 1994, 'Lévy statistics in a Hamiltonian system'. Phys. Rev. E 49, 4873-4877.

Kominis, Y., A. K. Ram y K. Hizanidis: 2010, 'Kinetic Theory for Distribution Functions of Wave-Particle Interactions in Plasmas'. Physical Review Letters 104(23), 235001.

Kook, H.-T. y J. D. Meiss: 1990, 'Diffusion in symplectic maps'. Phys. Rev. A 41, 4143-4150.

Kozlov, V. V.: 1983, 'Integrability and non-integrability in Hamiltonian mechanics'. Russ. Math. Surv. 38, 1-76.

Lega, E., M. Guzzo y C. Froeschlé: 2003, 'Detection of Arnold diffusion in Hamiltonian systems'. Physica D 182, 179-187.

Lichtenberg, A. J. y M. A. Lieberman: 1992, Regular and chaotic dynamics. New York: Springer-Verlag, second edition.

Lissauer, J. J.: 1995, 'Urey prize lecture: On the diversity of plausible planetary systems'. ICARUS 114, 217-236.

Lochak, P.: 1999, 'Arnold diffusion; a compendium of remarks and questions'. In: C. Simó (ed.): Hamiltonian systems with three or more degrees of freedom, Vol. 533 of Series C: Mathematical and Physical Sciences.

Maffione, N. P., L. A. Darriba, P. M. Cincotta y C. M. Giordano: 2011, 'A comparison of different indicators of chaos based on the deviation vectors: application to symplectic mappings'. Celest. Mech. Dyn. Astron. 111, 285-307.

Mandelbrot, B. B. y J. W. van Ness: 1968, 'Fractional Brownian Motions, Fractional Noises and Applications'. SIAM Review 10, 422-437.

Meiss, J. D.: 1992, 'Symplectic maps, variational principles, and transport'. Rev. Mod. Phys. 64, 795-848.

Meiss, J. D., J. R. Cary, C. Grebogi, J. D. Crawford, A. N. Kaufman y H. D. I. Abarbanel: 1983, 'Correlations of periodic, area-preserving maps'. Physica D 6, 375-384.

Mel'nikov, V. K.: 1963. Sov. Math.-Doklady 4, 266.

Mestre, M., P. M. Cincotta y C. M. Giordano: 2009, 'Testing the accuracy of the overlap criterion'. Int. J. Nonlinear Mech. 44, 180-189.

Mestre, M. F.: 2006, 'Interacción de resonancias y transición al caos en un modelo dinámico simple'. Tesis de grado en astronomía, FCAGLP, UNLP, Argentina.

Mestre, M. F., P. M. Cincotta y C. M. Giordano: 2011, 'Analytical relation between two chaos indicators: FLI and MEGNO'. MNRAS 414, L100-L103.

Metzler, R., A. V. Chechkin y J. Klafter: 2007, 'Lévy Statistics and Anomalous Transport: Lévy flights and Subdiffusion'. ArXiv e-prints.

Morbidelli, A.: 2006, MODERN CELESTIAL MECHANICS - Aspects of Solar System Dynamics. Berlin Heidelberg: Taylor \& Francis/Cambridge Scientific Publishers. 
Morbidelli, A. y A. Giorgilli: 1995, 'Superexponential stability of KAM tori'. Journal of Statistical Physics 78, 1607-1617.

Mudryk, L. R. y Y. Wu: 2006, 'Resonance Overlap Is Responsible for Ejecting Planets in Binary Systems'. ApJ 639, 423-431.

Nekhoroshev, N. N.: 1977, 'An Exponential Estimate of the Time of Stability of NearlyIntegrable Hamiltonian Systems'. Russ. Math. Surv. 32, 1-65.

Novaković, B., K. Tsiganis y Z. Knežević: 2010, 'Chaotic transport and chronology of complex asteroid families'. MNRAS 402, 1263-1272.

Oseledec, V. I.: 1968, 'A multiplicative ergodic theorem. Liapunov characteristic numbers for dynamical systems'. Trans. Moscow Math. Soc. 19, 197-221.

Poincaré, H.: 1892, Les methodes nouvelles de la mecanique celeste. Paris: Gauthier-Villars et fils.

Prince, P. J. y J. R. Dormand: 1981, 'High order embedded Runge-Kutta formulae'. J. Comput. Appl. Math. 7(1), 67-75.

Rebenshtok, A. y E. Barkai: 2008, 'Weakly Non-Ergodic Statistical Physics'. J. Stat. Phys. 133, 565-586.

Robutel, P. y F. Gabern: 2006, 'The resonant structure of Jupiter's Trojan asteroids - I. Long-term stability and diffusion'. MNRAS 372, 1463-1482.

Robutel, P., F. Gabern y A. Jorba: 2005, 'The Observed Trojans and the Global Dynamics Around The Lagrangian Points of the Sun Jupiter System'. Celest. Mech. Dyn. Astron. 92, $53-69$.

Sacha, K. y J. Zakrzewski: 1997, 'H-atom ionization by elliptically polarized microwave fields: The overlap criterion'. Phys. Rev. A 56, 719-728.

Schlier, C. y A. Seiter: 1998, 'Symplectic Integration of Classical Trajectories:2̆009 A Case Study'. The Journal of Physical Chemistry A 102(47), 9399-9404.

Schlier, C. y A. Seiter: 2000, 'High-order symplectic integration: an assessment'. Comput. Phys. Commun. 130(1-2), $176-189$.

Siboni, S., G. Turchetti y S. Vaienti: 1994, 'Diffusion on the torus for Hamiltonian maps'. J. Stat. Phys. 75, 167-187.

Skokos, C.: 2001, 'Alignment indices: a new, simple method for determining the ordered or chaotic nature of orbits'. J. Phys. A-Math. Gen. 34, 10029-10043.

Skokos, C.: 2010, 'The Lyapunov Characteristic Exponents and Their Computation'. In: Lect. Notes Phys., Berlin Springer Verlag, Vol. 790. pp. 63-135.

Skokos, C., C. Antonopoulos, T. C. Bountis y M. Vrahatis: 2004, 'Detecting order and chaos in Hamiltonian systems by the SALI method'. J. Phys. A - Math. Gen. 37, 6269. 
Skokos, C., T. C. Bountis y C. Antonopoulos: 2007, 'Geometrical properties of local dynamics in Hamiltonian systems: The Generalized Alignment Index (GALI) method'. Physica D 231(1), 30-54.

Soskin, S. M. y R. Mannella: 2009, 'Maximal width of the separatrix chaotic layer'. Phys. Rev. E 80(6), 066212.

Soskin, S. M., R. Mannella, O. M. Yevtushenko, I. A. Khovanov, and P. V. E. McClintock: 2009, 'A New Approach To The Treatment Of Separatrix Chaos And Its Applications'. ArXiv e-prints.

Stratonovich, R. L. y A. Silverman: 1967, Topics in the theory of random noise: general theory of random processes, nonlinear transformations of signals and noise, No. v. 1 in Mathematics and its applications. Gordon and Breach.

Tennyson, J. L., M. A. Lieberman y A. J. Lichtenberg: 1980, 'Diffusion in near-integrable hamiltonian systems with three degrees of freedom'. In: American Institute of Physics Conference Series, Vol. 57. pp. 272-301.

Todorović, N., E. Lega y C. Froeschlé: 2008, 'Local and global diffusion in the Arnold web of a priori unstable systems'. Celest. Mech. Dyn. Astron. 102, 13-27.

Tsiganis, K., Z. Knežević y H. Varvoglis: 2007, 'Reconstructing the orbital history of the Veritas family’. ICARUS 186, 484-497.

Uhlenbeck, G. E. y L. S. Ornstein: 1930, 'On the Theory of the Brownian Motion'. Phys. Rev. 36, 823-841.

Čubrović, M.: 2005, 'Fractional kinetic model for chaotic transport in nonintegrable Hamiltonian systems'. Phys. Rev. E 72(2), 025204-+.

Valk, S., N. Delsate, A. Lemaître y T. Carletti: 2009, 'Global dynamics of high area-to-mass ratios GEO space debris by means of the MEGNO indicator'. Advances in Space Research 43, 1509-1526.

Varvoglis, H.: 2005, Chaos, random walks and diffusion in Hamiltonian systems, Chapt. 6. Advances in astronomy and astrophysics. Cambridge, UK: Cambridge Scientific Publishers.

Venegeroles, R.: 2008, 'Calculation of Superdiffusion for the Chirikov-Taylor Model'. Phys. Rev. Lett. 101(5), 054102.

Wisdom, J.: 1980, 'The resonance overlap criterion and the onset of stochastic behavior in the restricted three-body problem'. AJ 85, 1122-1133.

Zaburdaev, V., S. Denisov y P. Hänggi: 2011, 'Perturbation Spreading in Many-Particle Systems: A Random Walk Approach'. Phys. Rev. Lett. 106(18), 180601-+.

Zaslavski, G. M. y B. V. Chirikov: 1972, 'Reviews of Topical Problems: Stochastic Instability of Non-Linear Oscillations'. Soviet Physics Uspekhi 14, 549-568.

Zaslavsky, G. M.: 2002, 'Chaos, fractional kinetics, and anomalous transport'. Phys. Rep. 371, 461-580. 